\title{
Extracellular vesicles as mediators of intercellular communication in human breast cancer progression
}

\author{
Doctoral thesis \\ In partial fulfillment of the requirements for the degree \\ "Doctor rerum naturalium (Dr. rer. nat.)" \\ in the Molecular Medicine Study Program \\ at the Georg-August University Göttingen
}

submitted by

Kerstin Menck

born in Hannover

Göttingen 2014 


\section{Members of the Thesis committee:}

\section{Supervisor:}

Prof. Dr. Claudia Binder

Department of Hematology/Medical Oncology

University Medical Center Göttingen

Second member of the thesis committee:

Prof. Dr. Uwe-Karsten Hanisch

Department of Neuropathology

University Medical Center Göttingen

Third member of the thesis committee:

Prof. Dr. Mikael Simons

Department of Neurology, University of Göttingen

Max Planck Institute of Experimental Medicine, Göttingen

Date of Disputation: 


\section{AFFIDAVIT}

I hereby declare that I wrote my doctoral thesis entitled "Extracellular vesicles as mediators of intercellular communication in human breast cancer progression" independently and with no other sources and aids than quoted.

Göttingen, February 2014

(Signature) 


\section{List of publications}

\section{Original articles:}

Menck, K., Scharf, C., Bleckmann, A., Dyck, L., Wenzel, D., Pukrop, T., Binder, C., Klemm, F. Tumor-derived microvesicles mediate human breast cancer invasion through differentially glycosylated EMMPRIN, submitted and under review.

Menck, K., Behme, D., Reiling, N., Binder, C., Pukrop, T., Klemm, F. Isolation of human monocytes by double gradient centrifugation and their differentiation to macrophages in Teflon-coated cell culture bags (2014), J Vis Exp, in revision.

Menck, K., Klemm, F., Gross, J. C., Pukrop, T., Wenzel, D. Binder, C. Induction and transport of Wnt 5a during macrophage-induced malignant invasion is mediated by two types of extracellular vesicles (2013), OncoTarget 4(11) 2057-2066.

Rietkötter, E., Menck, K., Bleckmann, A., Farhat, K., Schaffrinski, M., Schulz, M., Hanisch, U.-K., Binder, C., Pukrop, T. Zoledronic acid inhibits macrophage/microglia-assisted breast cancer cell invasion (2013), OncoTarget 4(9) 1449-1460.

\section{$\underline{\text { Abstracts: }}$}

Menck, K., Pukrop, T., Schulz, M., Dyck, L., Binder, C., Klemm, F. Breast cancer invasion mediated by plasma membrane-derived microvesicles is EMMPRIN-dependent (2012), Onkologie 35 195-195.

Menck, K., Klemm, F., Schulz, M., Pukrop, T., Binder, C. Identification and further characterization of microparticle populations in microparticle-induced breast cancer invasion (2011), Onkologie 34 60-60. 


\section{List of contents}

Table of contents ........................................................................................................................ I

Acknowledgments.............................................................................................................................. VI

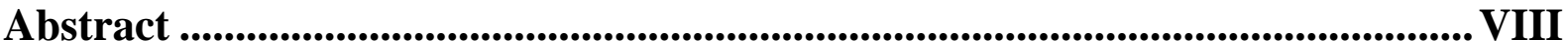

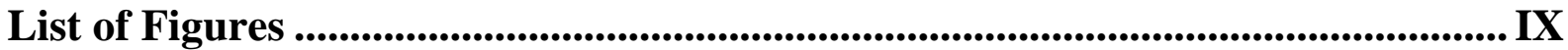

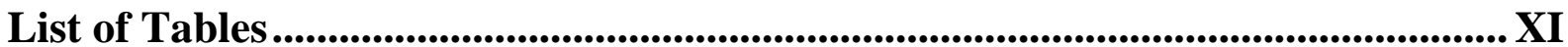

Abbreviations ....................................................................................................................XII

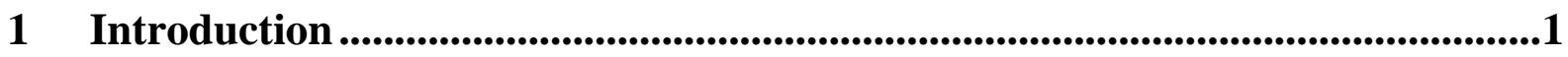

$1.1 \quad$ Breast cancer .................................................................................................................................. 1

1.2 Metastasis - one of the hallmarks in cancer progression ................................................... 2

$1.3 \quad$ Tumor microenvironment.............................................................................................................. 3

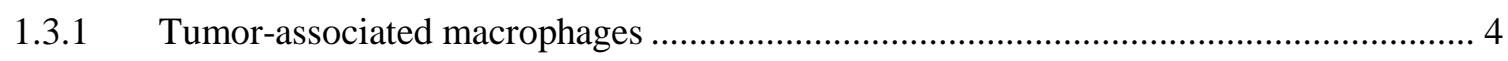

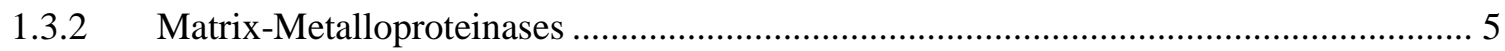

1.3.3 Extracellular Matrix Metalloproteinase Inducer (EMMPRIN) ….................................. 6

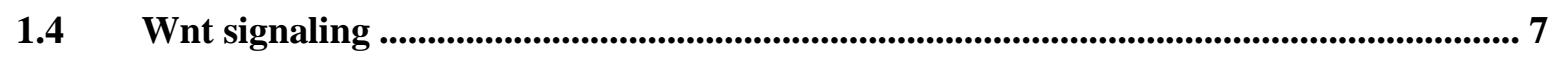

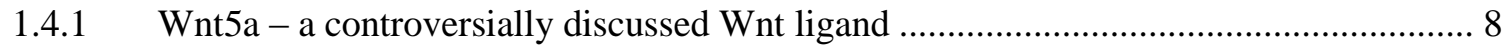

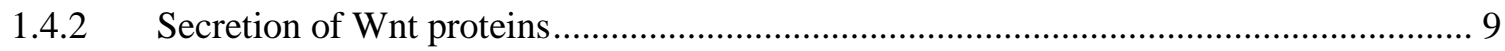

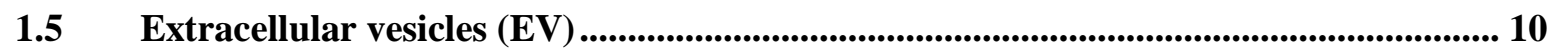

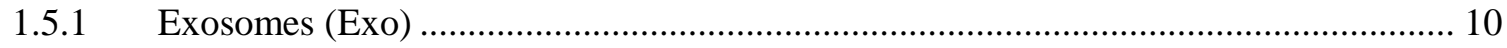

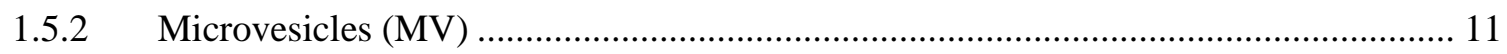

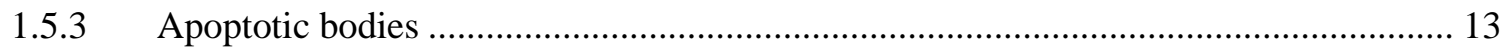

1.6 The significance of extracellular vesicles in cancer .......................................................... 14

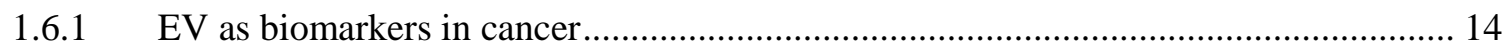

1.6.2 EV as mediators of intercellular communication in cancer.......................................... 14

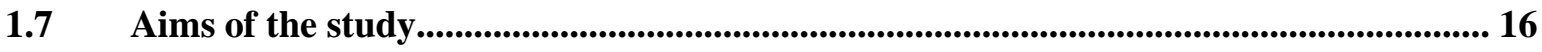

2 Materials and Methods .....................................................................................................18

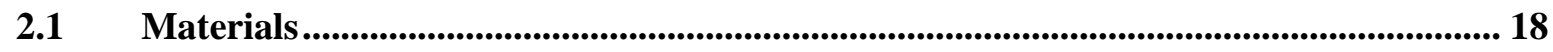

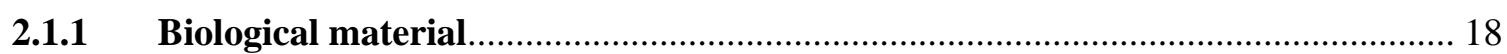




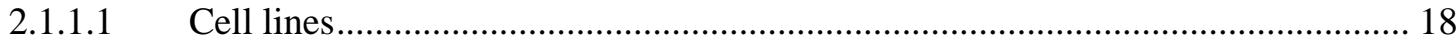

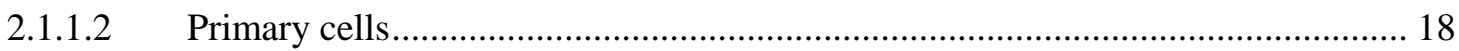

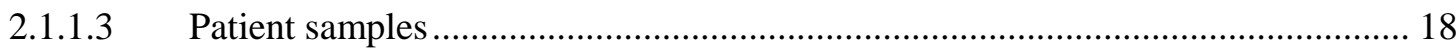

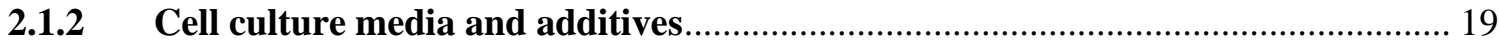

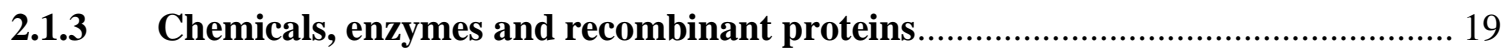

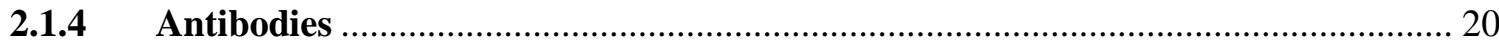

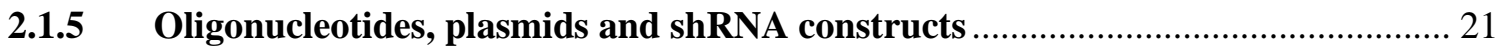

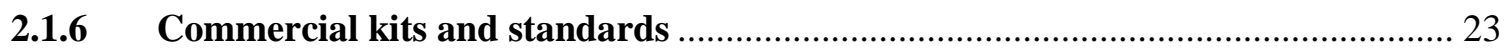

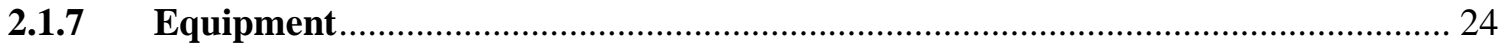

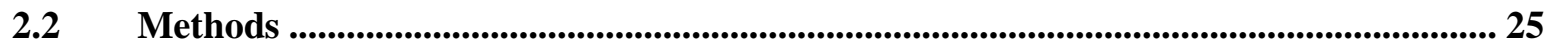

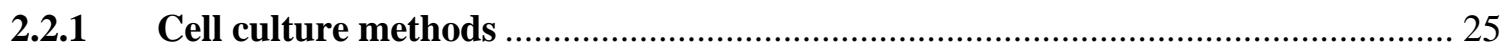

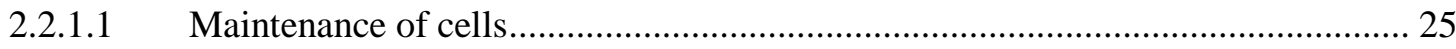

2.2.1.2 Generation of stable knockdown cell lines......................................................... 25

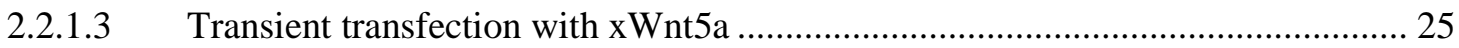

2.2.1.4 Isolation of human monocytes from Buffy Coats .............................................. 26

2.2.1.5 Differentiation of human monocytes to macrophages.......................................... 27

2.2.1.6 Generation of activated macrophages by stimulation with tumor cell supernatant 27

2.2.1.7 Assessment of cell vitality and proliferation ..................................................... 28

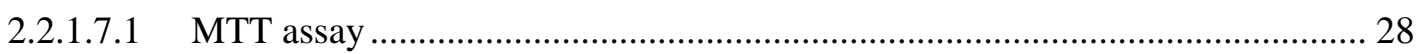

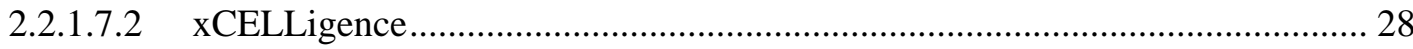

2.2.1.8 Cell invasion assay in a modified Boyden chamber............................................ 28

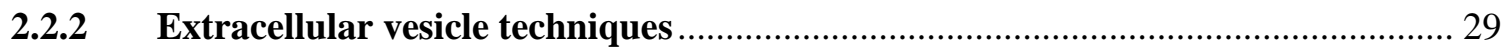

2.2.2.1 Isolation of microvesicles (MV) and exosomes (Exo) …………...................... 29

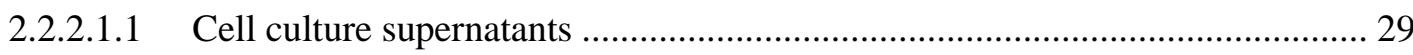

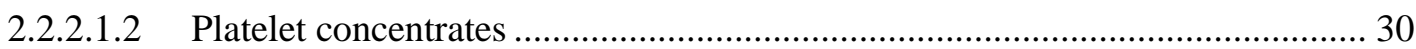

2.2.2.1.3 Peripheral blood from cancer patients and controls....................................... 30

2.2.2.2 Sucrose gradient ultracentrifugation .................................................................. 31

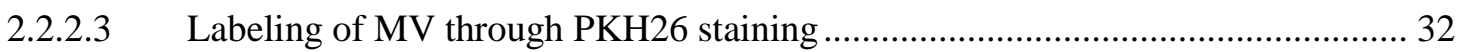

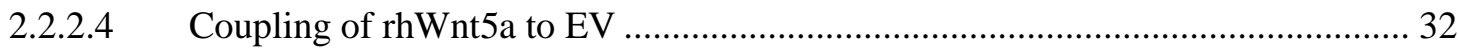

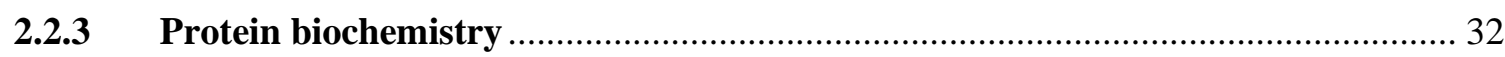




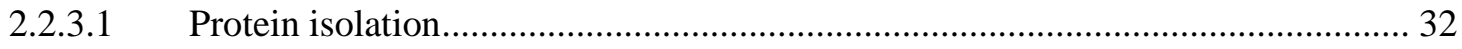

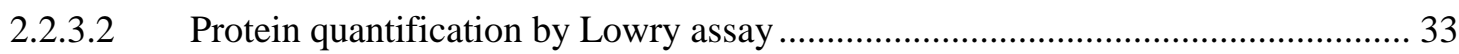

2.2.3.3 Protein deglycosylation by PNGaseF treatment .................................................... 33

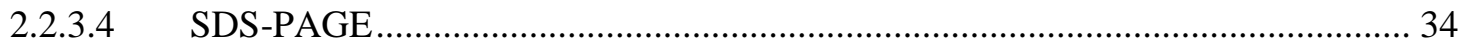

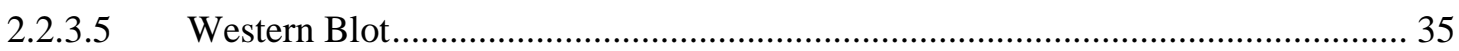

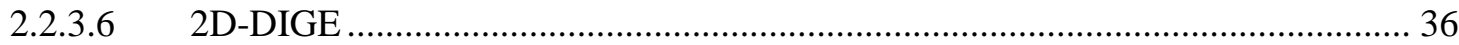

2.2.3.7 Analysis of the protein glycosylation status by 1D electrophoresis and mass

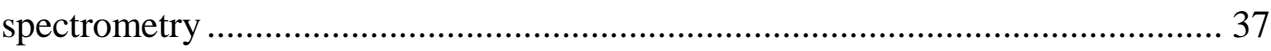

2.2.3.8 Detection of metalloproteinase activity by zymography .................................... 38

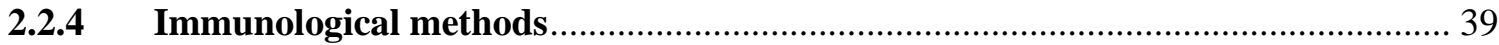

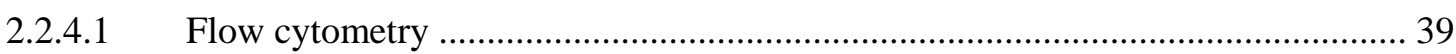

2.2.4.1.1 Characterization of MV by flow cytometry.................................................. 39

2.2.4.1.2 Characterization of Exo by flow cytometry .................................................... 39

2.2.4.1.3 Characterization of cells by flow cytometry ................................................. 40

2.2.4.1.4 Determination of MV counts by flow cytometry ........................................... 40

2.2.4.1.5 Measurement of MV uptake by flow cytometry........................................... 40

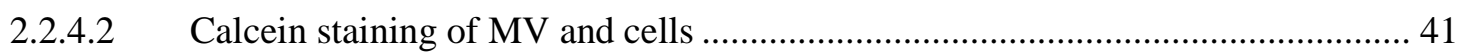

2.2.4.3 Separation of T-EV by MACS ……….............................................................. 41

2.2.4.4 Measurement of cytokine concentrations by ELISA ............................................. 41

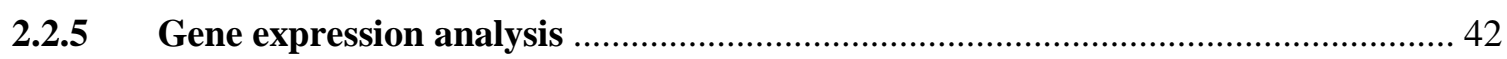

2.2.5.1 Isolation of total RNA from human tissue samples.............................................. 42

2.2.5.2 Isolation of mRNA from eukaryotic cells and vesicles ........................................ 43

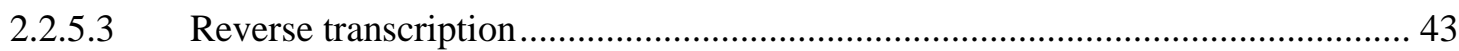

2.2.5.4 Quantitative real-time PCR (qRT-PCR) ........................................................... 44

2.2.5.4.1 Establishment of primers for qRT-PCR reactions ....................................... 45

2.2.5.4.2 Visualization of qRT-PCR products on agarose gels .................................... 46

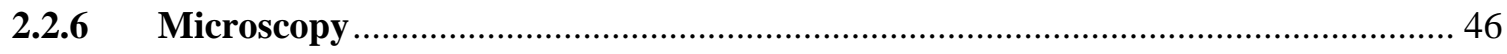

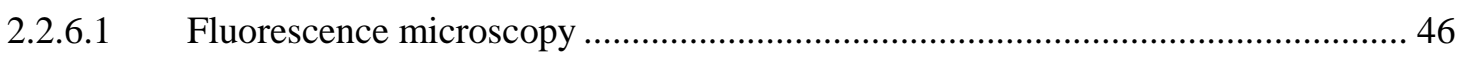

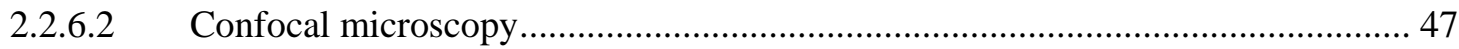

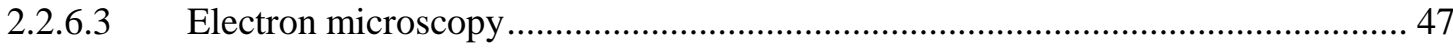


2.2.7 Statistical analysis 47

3 Results 48

3.1 EV as mediators of tumor-tumor interactions .............................................................. 48

3.1.1 Tumor cells release two distinct populations of extracellular vesicles ........................ 49

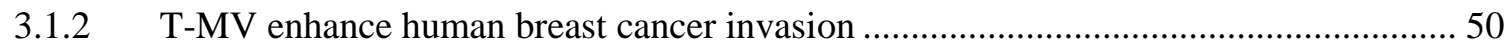

3.1.3 Uptake is essential, but not specific for pro-invasive MV ......................................... 52

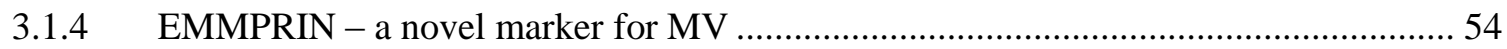

3.1.5 EMMPRIN is involved in the pro-invasive function of T-MV .................................... 55

3.1.6 The glycosylation status of EMMPRIN is important for its function ............................ 56

3.1.7 Identification of a novel EMMPRIN glycoform ...................................................... 59

3.1.8 EMMPRIN-carrying T-MV increase tumor invasion through activation of p38/MAPK . 61

3.2 EV as mediators of tumor-macrophage interactions.................................................. 64

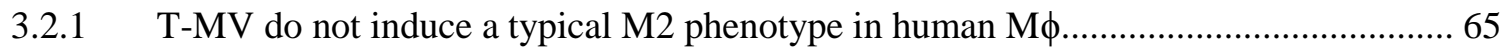

3.2.2 T-MV are responsible for the induction of Wnt5a in human $\mathrm{M} \phi \ldots \ldots \ldots \ldots \ldots \ldots \ldots \ldots \ldots \ldots . . . . . . . . . . . . . . . . . . .66$

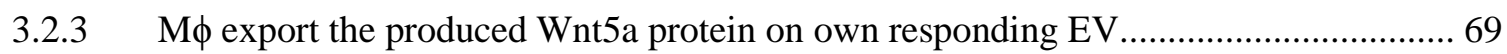

3.2.4 MV-induced Wnt5a expression is mediated through activation of p38/MAPK which can be antagonized by the Wnt-inhibitor DKK-1....................................................... 71

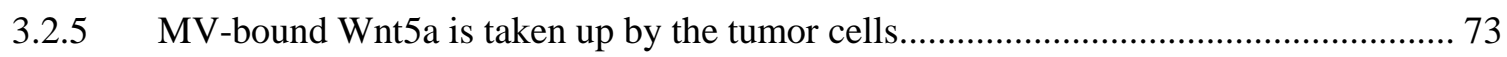

3.2.6 The role of Evi for Wnt5a expression on MV and Exo............................................... 74

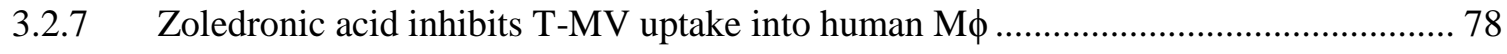

3.3 EV in human peripheral blood samples....................................................................... 79

3.3.1 Identification of MV-associated tumor markers by in vitro screening of T-MV .......... 79

3.3.2 EpCAM-based MACS of T-EV from human serum samples ...................................... 81

3.3.3 Establishment of a protocol for the isolation of MV from peripheral blood .................. 86

3.3.4 Isolation of EV from metastatic cancer patients........................................................ 90

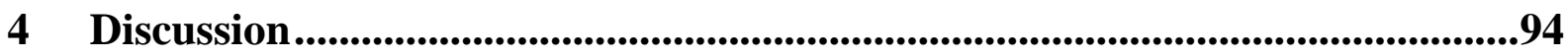

4.1 The role of EV in pro-invasive tumor-tumor crosstalk in breast cancer........................ 94

4.1.1 MV and Exo differ in their morphology and protein expression pattern ...................... 94 
4.1.2 T-EV enhance breast cancer progression by stimulating tumor cell invasion in an autologous and heterologous way

4.1.3 Glycosylation at N160 and N268 is essential for the pro-invasive effect of MVassociated EMMPRIN on tumor invasion 96

4.1.4 The pro-invasive function of EMMPRIN-carrying T-MV is independent of MMP ..... 98

4.2 The role of $\mathrm{EV}$ in Wnt5a induction and transport in $\mathrm{M} \phi$-induced breast cancer

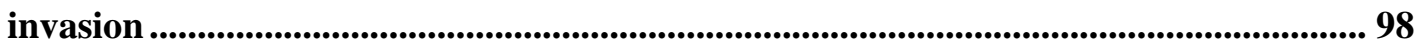

4.2.1 T-MV drive $\mathrm{M} \phi$ in a tumor-supporting phenotype by inducing Wnt5a ........................ 98

4.2.2 Wnt5a is transported on MV and supports tumor progression.................................. 100

4.3 EV in peripheral blood of metastatic cancer patients .................................................... 102

4.3.1 Isolation of T-EV from human serum samples ....................................................... 102

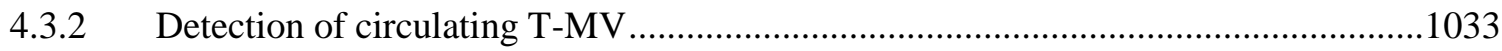

5 Summary and conclusions ...................................................................107

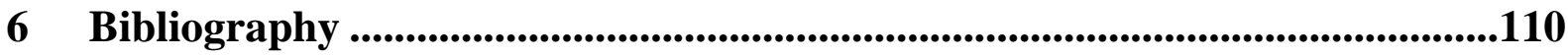

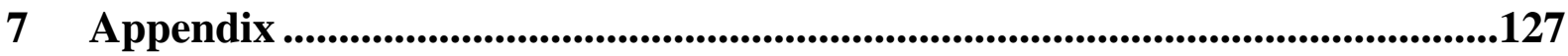

7.1 List of identified N-linked glycoproteins in 2D-DIGE gels ............................................ 127

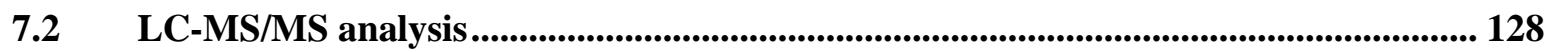




\section{Acknowledgements}

First of all, I would like to thank Prof. Claudia Binder for the supervision of the thesis and all the advice and discussions throughout the last three and a half years. Thank you for supporting my scientific education and giving me the opportunity to pursue my own hypotheses and especially for the chance to present our work at the Wnt congress in Heidelberg last year.

Furthermore, I am grateful for the support and advice of my second supervisor Dr. Florian Klemm who helped me to solve all the everyday problems in the lab and supervised the EMMPRIN studies. Thank you for the many constructive and inspiring discussions, especially during our rail travel tours to Greifswald.

I would also like to thank Prof. Uwe-Karsten Hanisch and Prof. Mikael Simons for participating in the thesis committee and all the helpful discussions which contributed to the successful completion of this thesis.

I gratefully acknowledge Dr. Tobias Pukrop for the constructive scientific discussions as well as the chance to contribute to the ZA project. Moreover, I thank Dr. Annalen Bleckmann for her help in collecting the patient blood samples and especially for her support regarding the analysis and statistics of the patient data.

Special thanks go to all our collaborators who participated in this study: Dr. Christian Scharf and Kathrin Darm from the University Medicine Greifswald for the proteomic studies and the identification of the EMMPRIN glycosylation pattern; Dr, Julia Gross from the German Cancer Research Center, Heidelberg, for the Wnt5a-L cells, the shEvi construct, the electron microscopy pictures of our tumor exosomes and the discussions regarding Wnt secretion; Dr Dirk Wenzel from the Max-Planck-Institute for Biophysical Chemistry, Göttingen, for visualizing our invisible MV pellets; Dr. Leila Siam for providing the neurosurgical resections of human brain metastases. 
To conduct my $\mathrm{PhD}$ thesis would not have been possible without the help of all my lab members: Thanks to Matthias for performing all the Boyden chamber experiments and the many EV isolations; Lena for establishing the knockdown cell lines for me and especially for the many delicious cakes and chocolate muffins; Meike for the millions of isolated macrophages; Eugenia for recording all the nice moments with her camera; Raquel and Jetcy for their great friendship in the lab and beyond. Last, but not least, I would like to thank my great colleague and friend Dr. Eva Rietkötter for all her technical and scientific support as well as, most important, for her encouragement and motivation (especially on Thursdays).

My special thanks go to my whole family, Karl-Ludwig, Simone, Almut, Sabine and especially Felix for always being there for me and providing me with their unconditional support and their persistent confidence in me which helped me my though all the hard times in the last three and a half years.

Finally, I would like to dedicate this thesis to my mother Waltraud. She was the first person to support my scientific career and always believed that I could reach all the goals I set for myself, even when no one else did. I am very proud to be her daughter. 


\begin{abstract}
The establishment of a permissive tumor microenvironment is a key step for tumor progression and metastasis formation. Tumor cells are known for their ability to create such a favorable tumor niche by influencing the surrounding benign stroma cells through the secretion of cytokines or growth factors. Recently, there is increasing evidence that also extracellular vesicles $(\mathrm{EV})$ released by the tumor cells are important means of intercellular communication. Therefore, we aimed to investigate their role in tumor-tumor and tumorstroma crosstalk in human breast cancer.
\end{abstract}

Human breast cancer cells were found to release tumor microvesicles (T-MV) as well as tumor exosomes (T-Exo). Both significantly enhanced invasiveness of the tumor cells in an auto- and heterologous feedback loop, whereas benign MV or the particle-free supernatant had no such effect. In case of T-MV, their pro-invasive function was dependent on a dynamin-dependent uptake into their recipient cells. Moreover, all pro-invasive T-MV carried a highly-glycosylated isoform of the Extracellular Matrix Metalloproteinase Inducer (EMMPRIN) with N-glycosylation at N160 and N268. Anti-EMMPRIN strategies such as knockdown or deglycosylation antagonized the pro-invasive function of T-MV on tumor invasion. Interestingly, EMMPRIN-carrying T-MV increased tumor invasiveness in a matrix metalloproteinase-independent mechanism by activating p38/MAPK signaling. EMMPRIN is not only involved in MV-mediated pro-invasive tumor-tumor crosstalk in vitro, but can also be used as a novel marker to detect T-MV which are circulating at high numbers in peripheral blood of breast cancer patients in vivo.

Apart from their autologous influence on tumor invasion, we identified tumor-EV (T-EV) as mediators of a reciprocal tumor-M $\phi$ communication loop. They were able to reprogram $\mathrm{M} \phi$ by inducing Wnt5a expression which was not observed for benign MV. Wnt5a induction was mediated by p38/MAPK signaling which could be antagonized by the Wnt-inhibitor DKK-1. $\mathrm{M} \phi$-Wnt5a was then exported on M $\phi$-derived EV and transported back to the tumor cells where it increased their invasive potential. Wnt5a expression on EV was shown to depend on the seven-pass transmembrane protein Evenness interrupted (Evi) which had been demonstrated so far only for exosomes and not for non-canonical Wnt ligands like Wnt5a.

In conclusion, breast cancer cells were demonstrated to release T-EV into the local environment as well as the bloodstream, where they interact with surrounding tumor as well as stroma cells and support the establishment of a favorable tumor niche. 


\section{List of Figures}

Fig. 1: Metastasis - a complex process of linked sequential steps................................................... 3

Fig. 2: Schematic representation of the EMMPRIN structure …....................................................... 6

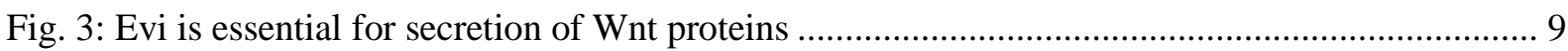

Fig. 4: Horizontal transfer of proteins and nucleic acids by EV ..................................................... 15

Fig. 5: Schematic representation of the modified Boyden chamber assay............................................ 29

Fig. 6: Schematic representation of the EV isolation protocol................................................................ 30

Fig. 7: MV and Exo are different populations of extracellular vesicles............................................... 49

Fig. 8: T-MV enhance tumor invasion in an autologous and heterologous way.................................. 51

Fig. 9: MV uptake does not differ between MV populations, but is important for their pro-invasive

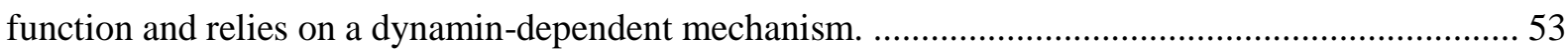

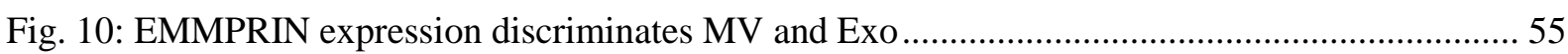

Fig. 11: Knockdown of EMMPRIN in SK-BR-3 cells reduces the pro-invasive effect of T-MV........ 56

Fig. 12: The pro-invasive effect of T-MV is mediated by HG-, and not IG-EMMPRIN .................... 57

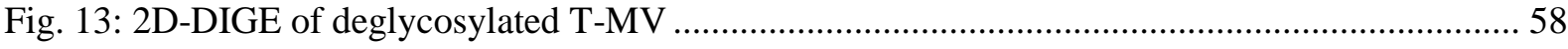

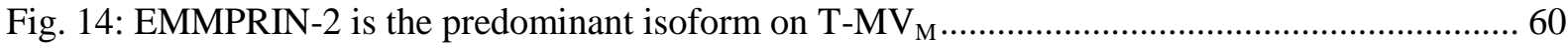

Fig. 15: Mass spectrometry reveals differential glycosylation of HG- and IG-EMMPRIN ................. 61

Fig. 16: T-MV have no effect on known EMMPRIN target genes in tumor cells ................................ 62

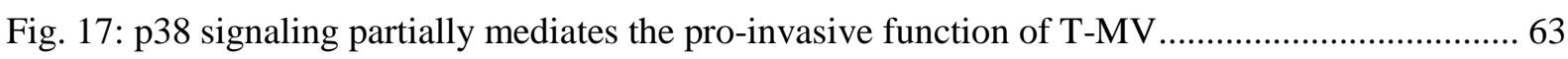

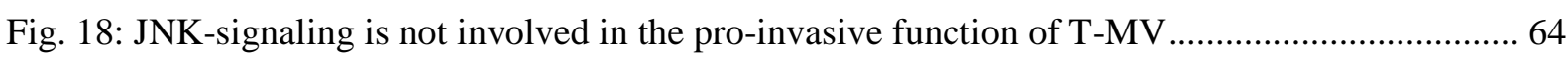

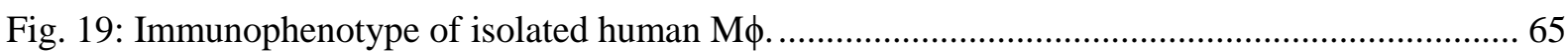

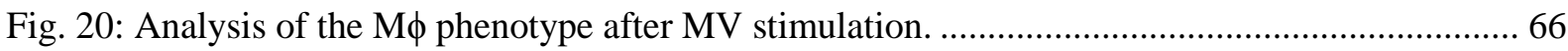

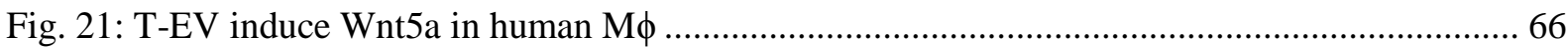

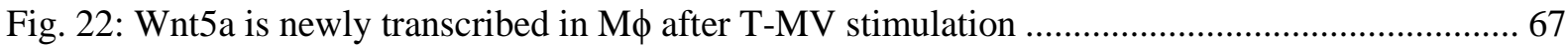

Fig. 23: Human M incorporate T-MV, but their uptake is not essential for Wnt5a induction............ 68

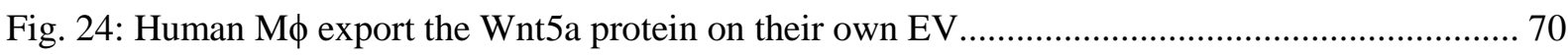

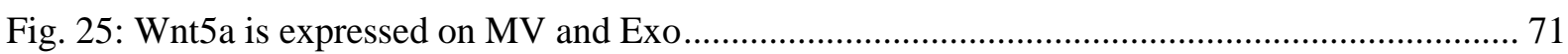

Fig. 26: MV-mediated Wnt5a induction in M $\phi$ is mediated by p38/MAPK signaling which can be

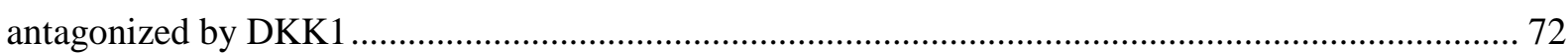

Fig. 27: Wnt5a-positive stroma-derived MV are ingested by breast cancer cells ............................... 73

Fig. 28: Wnt5a is associated with EV in a nonspecific as well as specific, Evi-dependent way .......... 75

Fig. 29: Implications of Evi knockdown for cellular Wnt5a expression.............................................. 76

Fig. 30: Knockdown of Evi impairs MV-mediated tumor invasion as well as the pro-invasive

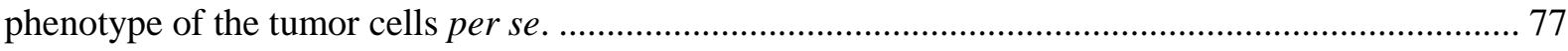

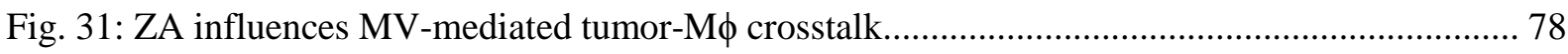

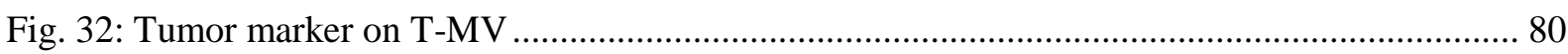

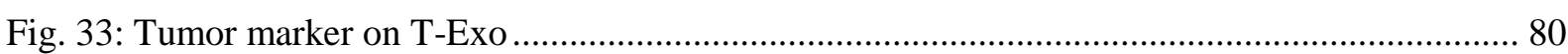




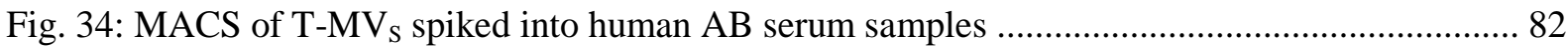

Fig. 35: LD, rather than LS, columns lead to higher MV yields in MACS of T-MV .......................... 83

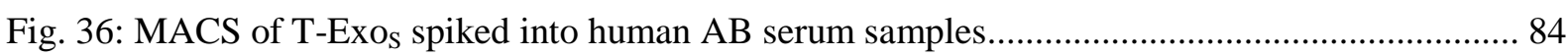

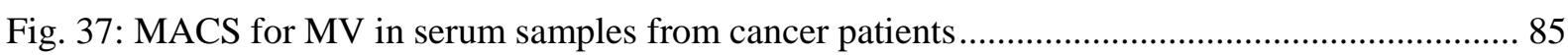

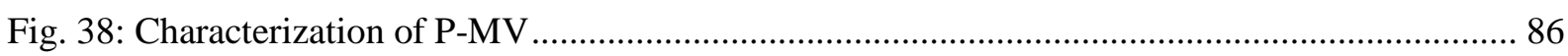

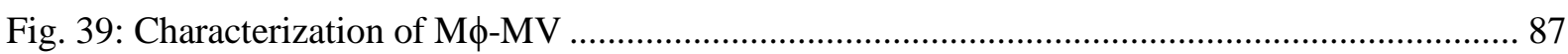

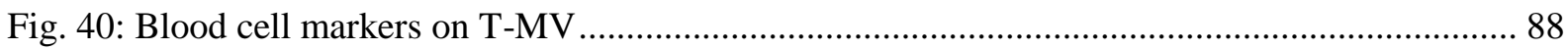

Fig. 41: Comparison of EDTA and heparin as anticoagulants for patient samples.............................. 89

Fig. 42: Isolation and characterization of EV from peripheral blood of cancer patients ...................... 90

Fig. 43: Tumor markers on MV derived from metastatic cancer patients............................................ 91

Fig. 44: Detection of T-MV in peripheral blood of metastatic breast cancer patients ......................... 92

Fig. 45: Schematic presentation of EV-mediated tumor-tumor crosstalk in human breast cancer ..... 107

Fig. 46: Schematic presentation of EV-mediated tumor-M $\phi$ crosstalk in human breast cancer ......... 108

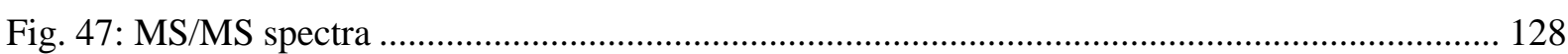




\section{List of Tables}

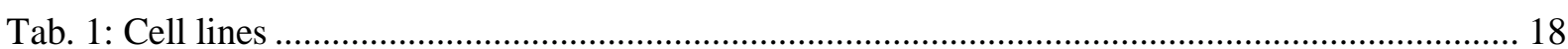

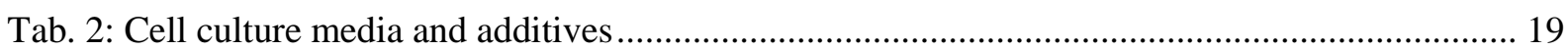

Tab. 3: Chemicals, enzymes and recombinant proteins ..................................................................... 20

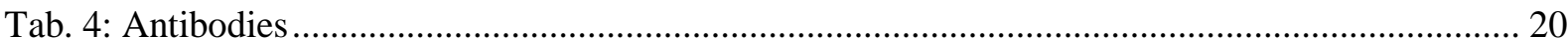

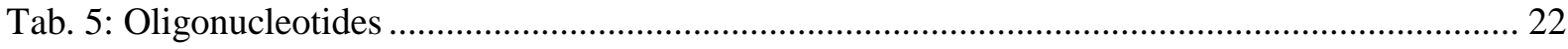

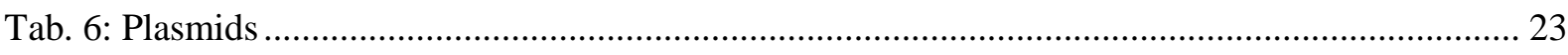

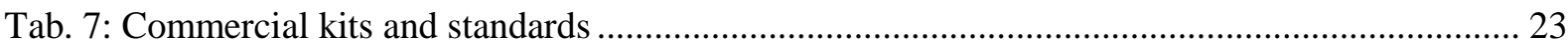

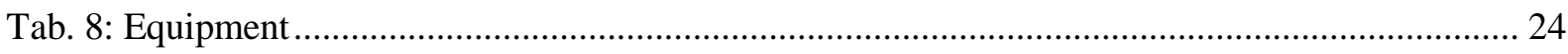

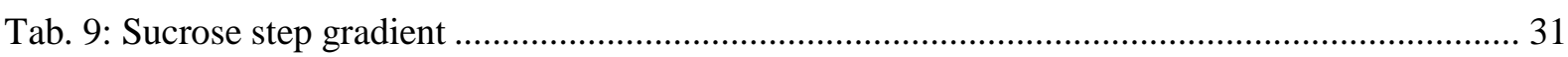

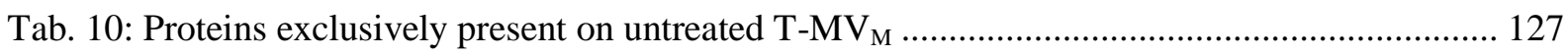

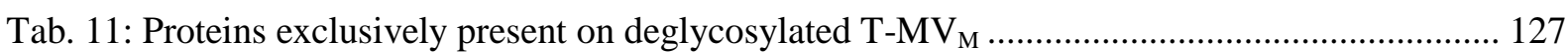




\section{Abbreviations}

2D-DIGE two-dimensional difference gel electrophoresis

CD cluster of differentiation

cDNA complementary DNA

DKK-1 Dickkopf-1

DMSO dimethyl sulfoxide

DNA deoxyribonucleic acid

ECM extracellular matrix

EDTA ethylenediaminetetraacetic acid

eGFP enhanced green fluorescent protein

EGFR epidermal growth factor receptor

EMMPRIN extracellular matrix metalloproteinase inducer

EpCAM epithelial cell adhesion molecule

ER estrogen receptor

ESCRT Endosomal Sorting Complexes Required for Transport

EV extracellular vesicles

Evi eveness Interrupted

Exo exosome(s)

FC flow cytometry

FCS fetal calf serum

FEP fluorinated ethylene propylene

Fig. figure

FITC fluorescein isothiocyanate

Her2 human epidermal growth factor receptor 2

HG highly glycosylated

HRP horseradish peroxidase

hTERT-MV microvesicles derived from hTERT-HME1 cells

IG intermediately glycosylated

IL interleukin

ILV intraluminal vesicles

IQR interquartile range

LC-MS/MS liquid chromatography-mass spectrometry/mass spectrometry

LG low glycosylated

L-MV microvesicles derived from murine L cells 
L-MV $\mathrm{Wnt5a}_{\text {a }}$ microvesicles derived from murine Wnt5a-overexpressing $\mathrm{L}$ cells

$\mathrm{L}^{-E \mathrm{Wn}_{\mathrm{Wn}} \mathrm{a}}$ exosomes derived from murine Wnt5a-overexpressing $\mathrm{L}$ cells

LPS lipopolysaccharide

M $\quad$ human macrophage(s)

M $\phi$-MV microvesicles derived from human macrophages

M $\phi$-Exo exosomes derived from human macrophages

MACS magnetic-activated cell sorting

MEGM mammary epithelial cell growth medium

MMP matrix metalloproteinases

mRNA messenger RNA

MTT 3-(4,5-Dimethylthiazol-2-yl)-2,5-diphenyltetrazolium bromide

MUC1 mucin 1

MV microvesicle(s)

MVB multivesicular bodies

PAGE polyacrylamide gel electrophoresis

PBS phosphate-buffered saline

PCP planar cell polarity

PE phycoerythrin

P-MV platelet-derived microvesicles

PNGaseF Peptide-N-Glyosidase F

PR progesterone receptor

qRT-PCR quantitative real-time polymerase chain reation

rh recombinant human

RNA ribonucleic acid

RUNX1 runt-related transcription factor 1

SD standard deviation

SDS sodium dodecyl sulfate

SEM standard error of the mean

shRNA small hairpin RNA

sn supernatant

Tab. table

TBST Tris-buffered saline with Tween 20

TEM transmission electron microscopy

T-EV tumor-derived extracellular vesicles 
T-Exo tumor-derived exosomes

T-Exо ${ }_{\mathrm{M}}$ exosomes derived from MCF-7 breast cancer cells

T-Exos exosomes derived from SK-BR-3 breast cancer cells

TLR Toll-like receptor

T-MV tumor-derived microvesicles

$\mathrm{T}-\mathrm{MV}_{\mathrm{M}} \quad$ microvesicles derived from MCF-7 breast cancer cells

$\mathrm{T}_{-} \mathrm{MV}_{\mathrm{S}} \quad$ microvesicles derived from SK-BR-3 breast cancer cells

Tsg101 tumor susceptibility gene 101

WB Western Blot

wt wild type

ZA zoledronic acid

\section{Measurement units:}

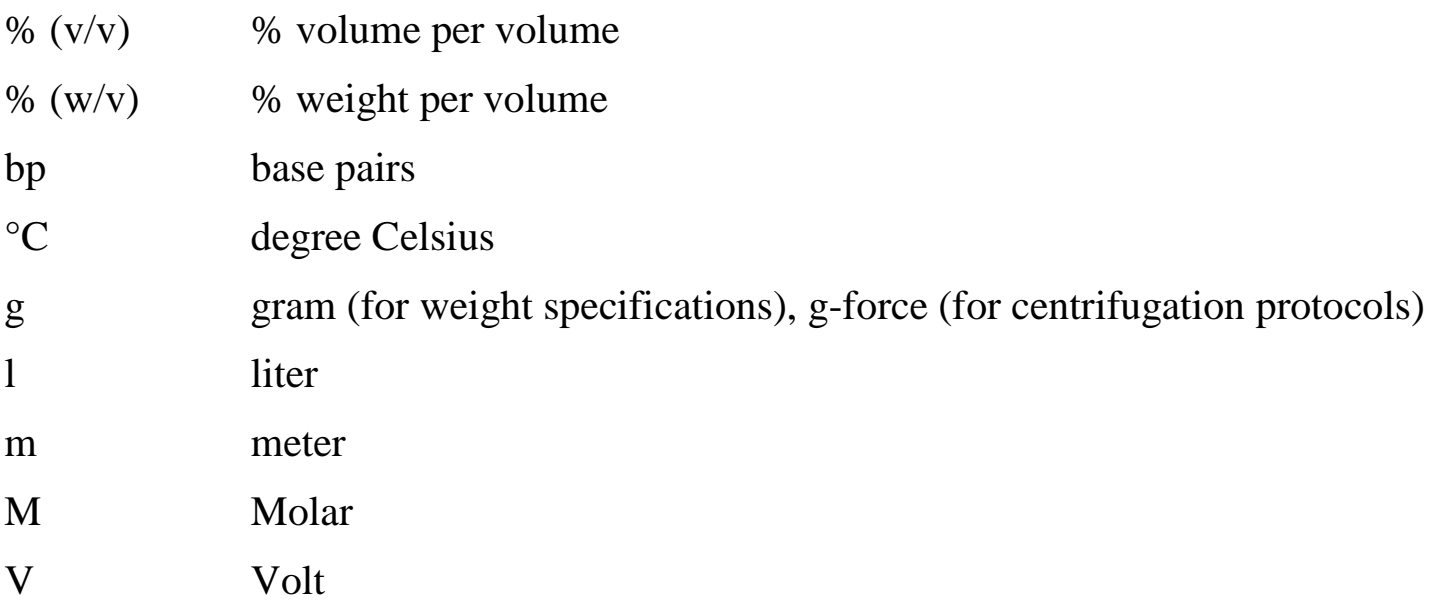

Metric prefixes:

$\begin{array}{ll}\mathrm{k} & \text { kilo; } 10^{3} \\ \mathrm{c} & \text { centi; } 10^{-2} \\ \mathrm{~m} & \text { milli; } 10^{-3} \\ \mu & \text { micro; } 10^{-6} \\ \mathrm{n} & \text { nano; } 10^{-9}\end{array}$




\section{Introduction}

\subsection{Breast cancer}

Breast cancer is the most common malignancy of women in the Western world and the second most common cancer worldwide after lung cancer (Hutchinson, 2010). More than 95\% of breast cancers are carcinomas meaning that they arise from transformed epithelial cells (Tomaskovic-Crook et al, 2009). While 5-6\% of breast cancers are considered hereditary mostly caused by mutations in the BRCA1 and BRCA2 gene (Malone et al, 1998), also environmental factors and especially estrogens are considered to support the onset of the disease (Hilakivi-Clarke, 2000). Based on microarray profiling, three major subtypes can be defined (Perou et al, 2000) which also differ in clinical outcome of the patients (Sorlie et al, 2001): Hormone receptor-positive, Her2/neu-positive and triple-negative breast cancers.

The majority of breast cancers (around 70\%) express the estrogen receptor (ER) accompanied by a varying expression of the progesterone receptor (PR) and are therefore considered as hormone-receptor positive (Dunnwald et al, 2007). They are mainly found in older women and are associated with better prognosis compared to the other two subtypes (Dunnwald et al, 2007). While primary systemic chemotherapy in these patients depends on individual risk assessment, all patients with detectable ER expression are treated adjuvantly with endocrine therapy (e.g. tamoxifen) (Senkus et al, 2013) which counteracts the stimulating effects of estrogen on cancer cell growth and has a response rate of around $80 \%$ in metastatic patients positive for both ER and PR (Higgins \& Stearns, 2009).

Between 15 to $30 \%$ of breast cancers are characterized by overexpression of Her2, usually due to amplification of the ERBB2 gene (Slamon et al, 1987). Although they generally have a poor outcome, recent developments of new anti-Her2 treatment strategies such as the monoclonal antibody trastuzumab have improved prognosis (Dawood et al, 2010; Saini et al, 2011). The third breast cancer subgroup comprises tumors that are ER-, PR- and Her2negative and are therefore referred to as triple-negative or basal-like. This type of tumor is found more often in younger women and although tumors initially respond to chemotherapy, they often relapse and thus are associated with a poor prognosis (Dunnwald et al, 2007).

Up to $15 \%$ of all breast cancer patients present with aggressive disease and develop metastases within 3 years after diagnosis (Weigelt et al, 2005). These metastases are preferentially formed in the liver, lung, brain or bone (Lee, 1983). Unfortunately, even after 
reaching a complete remission during primary treatment, there is a prolonged risk for recurrence of the disease, mainly in the form of metastases, for up to 20 years (McKay \& Langlands, 1992). In order to monitor therapeutic response and detect disease progression, several serum markers have been proposed. Among them is the epithelial transmembrane glycoprotein MUC1, also known as CA 15-3, which is the most widely used serum marker in breast cancer and was also shown to have prognostic value (Duffy et al, 2000). Moreover, it has already been used as a marker to detect tumor cells in peripheral blood of metastatic breast cancer patients (Alix-Panabieres et al, 2005). Other markers tested for the detection of circulating breast cancer cells are CK19, CK20, CAE, EGFR or Her2 (Gilbey et al, 2004). However, up to date no reliable, routinely applicable test to monitor disseminated tumor cells in peripheral blood is available and only sparse data regarding the prognostic value of individual markers exist.

\subsection{Metastasis - one of the hallmarks in cancer progression}

Metastasis describes the spreading of tumor cells to distant organs or parts of the body leading to the formation of secondary tumors. It was defined as one of the six hallmarks of cancer by Hanahan and Weinberg (Hanahan \& Weinberg, 2000). The occurrence of metastases often confers a devastating clinical fate because more than $90 \%$ of cancer-related deaths are due to metastases which are often resistant to therapy (Weigelt et al, 2005). Metastasis consists of several sequential steps which are displayed in Fig. 1 and can be stopped at multiple stages when one of these steps is not completed successfully (Poste \& Fidler, 1980).

Acquisition of an invasive phenotype is the first step for metastatic tumor progression which leads to local degradation of the surrounding tissue at the primary tumor site completed by breakdown of the basal membrane (Weber, 2008). This enables the tumor cells to spread throughout the body on three major paths including lymphatic vessels, blood vessels or along surfaces across body cavities (Stacker et al, 2002). Through stimulation of neovascularization, even small tumors $(<2 \mathrm{~mm}$ diameter) have access to the vasculature (Dvorak, 1986). After successful intravasation, circulating tumor cells must attach to the parenchyma of distant tissues to invade into and colonize these sites. These last steps are considered as highly inefficient and rate-limiting since from an estimated amount of up to $1 \cdot 10^{6}$ tumor cells which can be shed daily per gram of tumor mass (Butler \& Gullino, 1975), only less than $0,01 \%$ of these circulating tumor cells succeed in the establishment of a metastatic lesion (Fidler, 1970). 
Interestingly, different types of cancers seem to favor particular organs as metastatic sites as mentioned in chapter 1.1 for breast cancer. This is in line with the "Seed and Soil" hypothesis which was already formulated in 1889 by the English surgeon Stephen Paget. It comprises the idea that different cancer cells (= the "seed") have an affinity for certain organs (= the "soil") because they offer a compatible microenvironment which allows tumor growth (Paget, 1889).

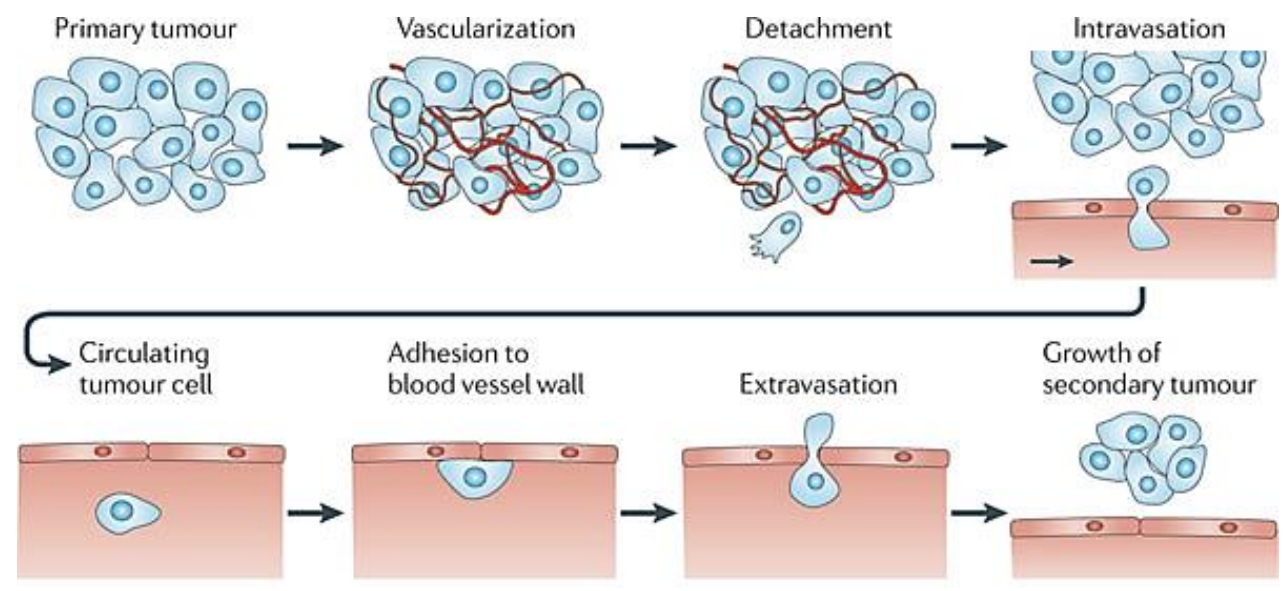

Fig. 1: Metastasis - a complex process of linked sequential steps

Metastasis is a complex multi-step process which begins in a vascularized primary tumor with the local invasion of tumor cells. After breakdown of the basement membrane, tumor cells detach from the tumor and spread throughout the body in blood and lymphatic vessels. Adhesion to blood vessel walls followed by extravasation of cancer cells into the parenchyma of distant organs paves the way for subsequent colonization of the tissue and formation of secondary tumors. Taken from (Wirtz et al, 2011) with permission from the Nature Publishing Group.

This idea still holds truth today. While a lot of research is focused on the intrinsic characteristics of tumor cells which drive oncogenic transformation and allow subsequent metastasis of these cells, the role of the tumor microenvironment for cancer progression is increasingly recognized (Joyce \& Pollard, 2009).

\subsection{Tumor microenvironment}

Malignant tumors consist not only of the transformed cancer cells, but grow in a particular microenvironment which comprises benign surrounding stroma cells including e.g. fibroblasts, muscle cells, endothelial and immune cells (Bissell \& Radisky, 2001). Moreover, also non-cellular components like cytokines, growth factors and especially the extracellular matrix (ECM) are major components of the local microenvironment. The ECM has a unique composition in each tissue which is highly influenced by reciprocal interactions between the different stroma cells (Frantz et al, 2010). 
Although normal stroma can counteract or even block tumor progression, exactly the opposite effect can be observed when the stroma is altered at the site of tumor growth (Bissell \& Radisky, 2001). During the last years it became increasingly apparent that tumor cells are able to influence the surrounding stroma cells to create a favorable tumor niche which is required for successful establishment of metastatic sites (Joyce \& Pollard, 2009; Qian \& Pollard, 2010). One of the pro-invasive factors produced by the tumor cells is the pro-inflammatory mediator $\mathrm{TNF} \alpha$, shown to induce expression of adhesion molecules on endothelial cells (Mannel et al, 1994; Stoelcker et al, 1995) thereby supporting adhesion and migration of tumor cells. Moreover, TNF $\alpha$ was demonstrated to impair NK cell activity which might represent a possible mechanism against anti-tumor immunity (Hafner et al, 1996). Apart from TNFa, also expression of VEGF by the tumor cells themselves (Aonuma et al, 1999) or associated stroma cells e.g. fibroblasts or macrophages (Decaussin et al, 1999; Lin et al, 2006) favors tumor progression by inducing angiogenesis.

\subsubsection{Tumor-associated macrophages}

Macrophages $(\mathrm{M} \phi)$ are often found at high numbers in tumor infiltrates (Pollard, 2004). In some breast cancer they even constitute more than $50 \%$ of the tumor mass (Lewis \& Pollard, 2006). Although immune cell infiltrates were first considered a failed attempt of the body to combat the tumor cells, it is now known that tumor cells can specifically attract and manipulate these cells to assist in tumor progression (Joyce \& Pollard, 2009). In line with this, infiltrating $\mathrm{M} \phi$ were detected especially at the invasive front of mammary tumors in human samples as well as in mouse models (Pukrop et al, 2006; Wyckoff et al, 2007). The number of tumor-associated $\mathrm{M} \phi$ was found to correlate with poor prognosis (Shih et al, 2006). In contrast, $\mathrm{M} \phi$ depletion by knockdown of macrophage colony-stimulating factor (M$\mathrm{CSF} / C S F 1$ ) or treatment with clodronate inhibited tumor growth and metastasis in mouse models (Hiraoka et al, 2008; Lin et al, 2001) which supports the hypothesis that $\mathrm{M} \phi$ exert protumoral functions in cancer progression.

$\mathrm{M} \phi$ differentiate from blood monocytes which are recruited to inflammatory or neoplastic sites (Mantovani et al, 1992). They are known as extremely heterogeneous cell population which interacts with the surrounding microenvironment and reacts to certain stimuli with phenotypic changes as well as specialized and polarized effector functions (Gordon, 2003; Mosser, 2003). In an attempt to group $\mathrm{M} \phi$ phenotypes, Mantovani defined two major $\mathrm{M} \phi$ subtypes in 2004: On the one hand, classically-activated, pro-inflammatory M1 M $\phi$ which develop upon exposure to Interferon- $\gamma$ or microbial stimuli like lipopolysaccharide (LPS) and, 
on the other hand, anti-inflammatory $\mathrm{M} 2 \mathrm{M} \phi$ which differentiate in the presence of Interleukin (IL) -4 and IL-13, IL-10 or exposure to immune complexes (Mantovani et al, 2004). M1 M $\phi$ characteristically express high levels of IL-12 and IL-23 and are known to induce a Th1-directed adaptive immune response with anti-microbial and anti-tumor activity. In contrast, M2 M $\phi$ display high levels of IL-10, IL-1 ra and IL-1 decoy receptor as well as low IL-12 expression and mediate immunosuppressive functions and tissue repair (Solinas et al, 2009). Tumor-associated $\mathrm{M} \phi$ have been described to acquire a tumor-supporting M2polarized phenotype when infiltrating the tumor stroma (Mantovani et al, 2002).

In a mouse model of mammary tumorigenesis $M \phi$ have been identified as source of growth factors, cytokines and matrix-degrading enzymes including matrix metalloproteinases (MMP) which mediated tumor progression (Lin et al, 2006). Up to now many studies have revealed pro-invasive tumor-M $\phi$ interactions. For instance, reciprocal signaling was demonstrated for breast cancer cells which stimulate infiltrated M $\phi$ by secretion of CSF1 and these respond with production of EGF that stimulates cancer cell growth and intravasation (Goswami et al, 2005; Qian \& Pollard, 2010; Wyckoff et al, 2007). Moreover, co-culture of benign breast cancer cells with human $\mathrm{M} \phi$ significantly increases invasiveness of the tumor cells which is mediated by MMP and is dependent on the expression of the Extracellular MMP Inducer (EMMPRIN) in the tumor cells (Hagemann et al, 2004; Hagemann et al, 2005). In addition, co-culture induces the expression of the pro-invasive factor Wnt5a in $\mathrm{M} \phi$ which is critical for the pro-invasive crosstalk that can be antagonized by the Wnt-inhibitor DKK-1 (Pukrop et al, 2006).

\subsubsection{Matrix-Metalloproteinases}

The MMP family comprises more than 21 endopeptidases that are capable of degrading virtually all proteins of the ECM (Egeblad \& Werb, 2002). They are secreted as inactive zymogens and need to be activated by proteolytic cleavage in order to be fully functional (Sternlicht \& Werb, 2001). The coordinated balance between secretion of MMP by stromal fibroblasts and expression of tissue inhibitors of MMP (TIMP) and other enzymes is crucial for normal tissue homeostasis (Cruz-Munoz \& Khokha, 2008).

The significance of MMP for tumor progression is due to the fact that they help tumor cells to remodel and degrade the ECM which facilitates cancer cell migration and invasion (Egeblad \& Werb, 2002). ECM degradation further leads to release of ECM-embedded tumorsupporting growth factors like e.g. VEGF or TGF- $\beta$ (Kessenbrock et al, 2010). Tumor cells 
were found to regulate stromal MMP expression via the Extracellular Matrix Metalloproteinase Inducer (EMMPRIN) (Jiang et al, 2001). However, MMP cannot only be secreted by stroma cells, but also have been reported to be released by the tumor cells themselves (Matrisian et al, 1986; Turpeenniemi-Hujanen et al, 1985).

\subsubsection{Extracellular Matrix Metalloproteinase Inducer (EMMPRIN)}

EMMPRIN, also known as CD147 or Basigin, is a cell surface transmembrane glycoprotein that belongs to the immunoglobulin superfamily (Biswas et al, 1995). It is encoded by the $B S G$ gene and consists of a $29 \mathrm{kDa}$ backbone protein which is known to be highly glycosylated. Three putative N-glycosylation sites have been described so far. Depending on the glycosylation status EMMPRIN appears in a size which can range from $32 \mathrm{kDa}$ up to $65 \mathrm{kDa}$ (Weidle et al, 2010). Four different splice variants of the protein have been identified with EMMPRIN-2 being the most abundant one (Liao et al, 2011). All four isoforms share a common C-terminal structure consisting of a 21 amino acid cytoplasmic tail, a 21 amino acid transmembrane domain and an extracellular Ig-like domain (Liao et al, 2011). In contrast to EMMPRIN-3 and -4, the main isoform EMMPRIN-2 additionally possesses a second and the retina-specific isoform EMMPRIN-1 a third Ig-like domain as shown in Fig. 2.

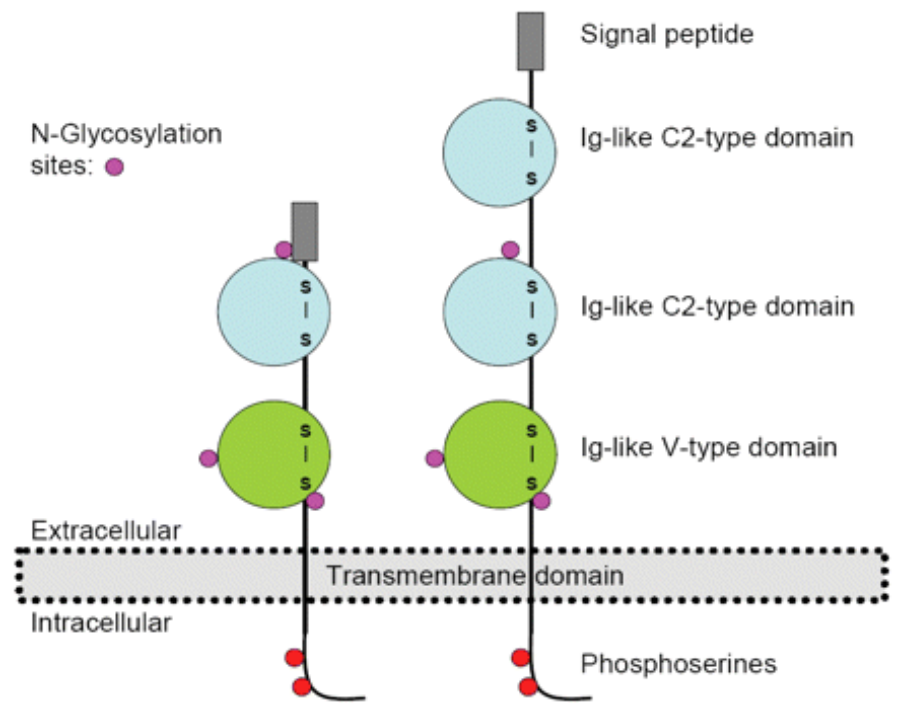

Fig. 2: Schematic representation of the EMMPRIN structure

The topology of the main isoform EMMPRIN-2 is displayed on the left. Next to the cytoplasmic tail and the transmembrane domain, it contains two extracellular Ig-like domains as well as a signal peptide sequence (21 amino acids) at the $\mathrm{N}$-terminus. In comparison, the retina-specific isoform EMMPRIN-1 which is shown on the right harbors a third extracellular Ig-like domain due to transcription of an additional exon. The three potential $\mathrm{N}$-glycosylation sites are indicated in purple, the phosphoserine residues as putative phosphorylation sites in red. Taken from (Weidle et al, 2010) with permission of the International Institute of Anticancer Research. 
The name EMMPRIN comes from the observation that EMMPRIN-expressing tumor cells as well as their supernatants have been shown to induce various MMP in stroma cells e.g. fibroblasts, endothelial cells or M $\phi$ (Biswas et al, 1995; Guo et al, 1997; Kataoka et al, 1993; Muralidharan-Chari et al, 2010). Furthermore, it was shown to be capable of inducing expression of the pro-invasive factors CSF1, TNFa or VEGF (Schmidt et al, 2008b; Seizer et al, 2010; Tang et al, 2005). Homophilic interactions of EMMPRIN molecules on opposing cells were described to stimulate EMMPRIN expression itself in a positive feedback loop (Sun \& Hemler, 2001; Tang et al, 2004b). In Lu-16 fibroblasts stimulation with purified EMMPRIN activates p38 signaling which can be blocked by the p38-inhibitor SB-203580 and resulted in decreased MMP induction (Lim et al, 1998).

A tumor-promoting role of EMMPRIN has been suggested due to observations that increased expression of EMMPRIN stimulates tumor growth and metastasis in vitro (Zucker et al, 2001) and was found to be the most frequently upregulated protein in transcriptome analysis of single micrometastatic cells in cancer patients in vivo (Klein et al, 2002). Moreover, EMMPRIN was found overexpressed in many different types of cancer and high expression correlated with poor prognosis (Nabeshima et al, 2006).

\subsection{Wnt signaling}

The Wnt signaling pathway plays a crucial role not only in cell-cell communication during development and normal tissue homeostasis, but its deregulation is also often associated with tumorigenesis and cancer progression. The more than 19 identified Wnt ligands belong to a family of highly conserved secreted glycoproteins and are capable of activating Wnt signaling (Gordon \& Nusse, 2006). During B-catenin-dependent, canonical Wnt signaling binding of Wnt proteins to a receptor complex consisting of a Frizzled receptor and the co-receptor LRP5/6 (Tamai et al, 2000; Wehrli et al, 2000) induces stabilization and translocation of cytoplasmic B-catenin to the nucleus where it binds to LEF/TCF transcription factors and activates target gene expression (van de Wetering et al, 1997). Apart from the Wnt inhibitory factor-1 (WIF-1) and secreted Frizzled-Related Proteins (sFRP), the family of Dickkopf (DKK) proteins is known to inhibit Wnt signaling by binding to the co-receptor LRP6 (Semenov et al, 2008).

In addition to the canonical pathway, two $\beta$-catenin-independent non-canonical pathways have been described: The planar cell polarity (PCP) pathway and the $\mathrm{Wnt} / \mathrm{Ca}^{2+}$ pathway. Activation of the latter has been associated with an increase in intracellular calcium levels 
which leads to activation of PKC and CamK II (Kuhl et al, 2000) which, in turn, activate several nuclear transcription factors including e.g. NFKB or CREB (De, 2011). The Wnt/Ca ${ }^{2+}$ pathway was shown to play a pivotal role in cardiac development (Rao \& Kuhl, 2010) and was suggested to exert tumor-suppressing effects in human basal cell carcinoma (Nitzki et al, 2010).

In contrast, PCP signaling was described to enhance tumor cell migration and invasion in several human cancer models due to its regulatory influence on cytoskeletal organization and cell motility (Ripka et al, 2007; Weeraratna et al, 2002). Activation of the PCP pathway induces a signaling cascade that results in activation of JNK-signaling and ROCK-mediated actin reorganization (Wallingford \& Habas, 2005). Both non-canonical pathways, Wnt/Ca ${ }^{2+}$ and PCP, can be activated by the Wnt ligand Wnt5a (De, 2011; Yamanaka et al, 2002).

\subsubsection{Wnt5a - a controversially discussed Wnt ligand}

Wnt5a was originally described as a non-canonical ligand although recent data show that it is also capable of activating B-catenin-dependent canonical Wnt signaling (Mikels \& Nusse, 2006). In this context, its influence on canonical signaling seems to depend on the receptor context (Mikels \& Nusse, 2006).

The Wnt5a protein was found to be glycosylated and palmitoylated, the latter being essential for its function (Kurayoshi et al, 2007). Due to its high hydrophobicity, it can bind to the ECM which was suggested to be involved in formation of local gradients (Ai et al, 2003). The role of Wnt5a in cancer is controversially discussed (McDonald \& Silver, 2009; Pukrop \& Binder, 2008). On the one hand, it was shown to have no transforming activity (Olson \& Papkoff, 1994; Wong et al, 1994), was found to be downregulated on the mRNA level in neuroblastoma, leukemia or endometrial carcinoma compared to corresponding benign tissues (Blanc et al, 2005; Bui et al, 1997; Liang et al, 2003) and even inhibited cell migration and invasion in several cancer models (Dejmek et al, 2005; Jiang et al, 2013; Kremenevskaja et al, 2005). On the other hand, Wnt5a is overexpressed in many human cancers (Pukrop \& Binder, 2008) and was shown to induce expression of several pro-invasive factors including MMP (Prieve \& Moon, 2003), CD44 and vimentin (Dissanayake et al, 2007) or laminin $\gamma 2$ (Hanaki et al, 2012). Wnt5a was not only expressed in the tumor cells themselves, but especially in tumor-associated M $\phi$ (Pukrop et al, 2006; Smith et al, 1999), which were predominantly localized at the invasive front of the tumor (Pukrop et al, 2006). 


\subsubsection{Secretion of Wnt proteins}

Although the first Wnt gene was already isolated in 1982 (Nusse \& Varmus, 1982), the mechanism of Wnt protein synthesis and secretion is still not completely understood. Wnt proteins are generally modified by a series of posttranslational modifications which are important for their secretion and function. Especially palmitoylation was found to be crucial for the activity of Wnt proteins (Willert et al, 2003). After translation most Wnt proteins are lipid-modified by the acyltransferase Porcupine which is located in the membrane of the endoplasmic reticulum (Kadowaki et al, 1996; Willert et al, 2003). It was shown that Porcupine-mediated acetylation requires correct previous glycosylation of Wnt3a (Komekado et al, 2007) or Wnt5a (Kurayoshi et al, 2007) and missing glycosylation significantly impaired secretion of both Wnt proteins.

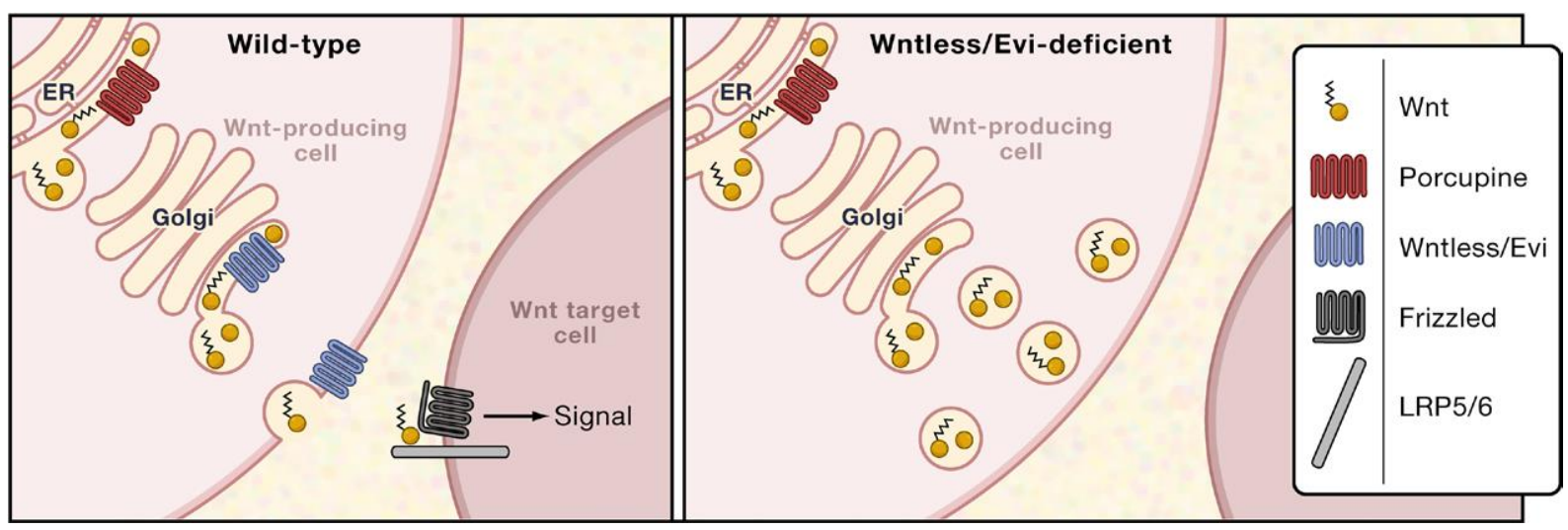

Fig. 3: Evi is essential for secretion of Wnt proteins

After lipid modification by the acyltransferase Porcupine in the endoplasmic reticulum, Wnt proteins are transported to the Golgi complex where they bind to Evi and are escorted to the cell surface in secretory vesicles for secretion (left). In contrast, loss of Evi results in an accumulation of synthesized Wnt proteins inside the cell (right). Taken from (Ching \& Nusse, 2006) with permission from Elsevier.

Lipid-modified Wnt proteins then bind to p24 proteins and are transported to the Golgi complex (Buechling et al, 2011; Port et al, 2011) where they associate with Evi, also known as WLS or Gpr177. Evi is a seven-pass transmembrane protein that is specifically required for escorting Wnt proteins from the Golgi complex to the plasma membrane (Banziger et al, 2006; Bartscherer et al, 2006). Loss of Evi causes an accumulation of Wnt proteins in the Wnt-producing cell (Bartscherer et al, 2006) as depicted in Fig. 3. Furthermore, Evi has often been proposed to exert a chaperone function for the correct folding of synthesized Wnt proteins, although this has not been confirmed yet. After secretion of Wnt proteins Evi is either targeted to lysosomal degradation or can be recycled via endosomes and the retromer 
complex to the Golgi where it can associate with new Wnt proteins and participate in a second round of Wnt secretion (Bartscherer \& Boutros, 2008; Port et al, 2008). Following secretion, Wnt proteins are found in the supernatant of the Wnt-producing cell, but were also predicted to be tethered to the cellular plasma membrane due to their hydrophobic lipid moieties (Janda et al, 2012).

\subsection{Extracellular vesicles (EV)}

Intercellular communication was always believed to be mediated by direct cell-cell interactions or via soluble mediators e.g. growth factors, hormones or cytokines. However, in the last years it became increasingly accepted that extracellular vesicles (EV) represent additional novel means of intercellular communication (Raposo \& Stoorvogel, 2013). Three different EV populations, including microvesicles, exosomes and apoptotic bodies, have been identified so far and shall be introduced in this chapter focusing on the differences in their biogenesis and composition.

\subsubsection{Exosomes (Exo)}

Exosomes (Exo) have been first described in 1987 in studies on reticulocyte maturation (Harding et al, 1983; Pan \& Johnstone, 1983) and are precipitated from cell culture supernatants or body fluids by ultracentrifugation at $100.000 \mathrm{~g}$ (Thery et al, 2006). They can be distinguished from microvesicles by electron microscopy due to their smaller size which ranges from 30-100 $\mathrm{nm}$ as well as their typically cup-shaped appearance (Thery et al, 2002). In order to further identify the nature of isolated vesicles from cell culture supernatants, they are typically loaded onto sucrose gradients where Exo float at densities ranging from 1.13$1,19 \mathrm{~g} / \mathrm{ml}$ (Hugel et al, 2005) which may vary from cell to cell depending on the Exo protein content (Stoorvogel et al, 2002; Thery et al, 2002).

In contrast to microvesicles which bud off from the cellular plasma membrane, Exo are derived from intraluminal vesicles (ILV) which are stored in multivesicular bodies (MVB). MVB are part of the endosomal compartment and originally contain internalized proteins from the cellular plasma membrane as well as proteins from the trans Golgi network which are destined for degradation (Gruenberg \& Stenmark, 2004). In general, MVB can be processed via two different routes: Either they fuse with lysosomes leading to degradation of their content or they fuse with the cellular plasma membrane and release their cargo of ILV into the extracellular space (exemplified in Fig. 4). These ILV are since that moment referred 
to as Exo (Gruenberg \& Stenmark, 2004). Transport to and docking of MVB at the plasma membrane with subsequent fusion is dependent on several Rab proteins including Rab-11 (Savina et al, 2005), Rab 27 (Ostrowski et al, 2010) and Rab 35 (Hsu et al, 2010) as well as intracellular calcium levels (Savina et al, 2005). It is a constant process (Thery et al, 2002), but can be regulated in some cell types by an increase of intracellular calcium (Raposo et al, 1997). ILV are formed by inward budding and subsequent fission from the limiting membrane of MVB (van Niel et al, 2006). For this reason, they contain endosomal MVB membrane proteins as well as cytoplasmic proteins which are engulfed by chance or because of transient interaction with an endosomal transmembrane protein. Protein sorting into ILV was described to be associated with the Endosomal Sorting Complexes Required for Transport (ESCRT), although also ESCRT-independent mechanisms seem to play a role (van Niel et al, 2006).

In line with this, Exo typically carry the two proteins Alix and Tsg101 (Thery et al, 2001) which belong to the ESCRT system (Katzmann et al, 2001; von Schwedler et al, 2003). Moreover, Exo express tetraspanin proteins including e.g. CD9, CD81 (Yoshioka et al, 2013) or CD63 (Fevrier \& Raposo, 2004). Since tetraspanins are known to be enriched in specialized membrane microdomains (Hemler, 2003), this may hint to the a specific protein sorting process from endosomal membranes into ILV (de Gassart et al, 2003). Typical marker proteins which are further associated with Exo are chaperones (e.g. HSP90, HSC70), flotillin1 (de Gassart et al, 2003) and flotillin-2 (Canfran-Duque et al, 2013; Strauss et al, 2010) or Rab proteins (e.g. Rab2, Rab7) (Fevrier \& Raposo, 2004). Exo were also found to express some proteins from the cell membrane. However, this seems to be a very selective process since some very abundant membrane proteins were not detected on exosomes (Blanchard et al, 2002; Thery et al, 2001)

\subsubsection{Microvesicles (MV)}

Microvesicles (MV) are a heterogeneous population of membrane vesicles with a diameter ranging from 100-1000 $\mathrm{nm}$ (Cocucci et al, 2009) and can be pelleted by a centrifugal force of 10.000 up to $20.000 \mathrm{~g}$ (van der Pol et al, 2012). Studies of pure MV populations are limited because they were originally pelleted together with Exo at $100.000 \mathrm{~g}$ which resulted in mixed populations of MV and Exo. Only recently, MV were accepted as distinct EV population and have been isolated apart from Exo. However, the purity of EV populations isolated by differential ultracentrifugation is questionable and so far there are no specific MV-associated markers known to reliably separate them from the other EV populations. In general, the 
expression pattern of cytosolic as well as membrane proteins transported on MV depends on their cell of origin as well as the process which triggers their release (VanWijk et al, 2003).

MV seem to be ubiquitously shed by all types of cells by outward budding of the cellular plasma membrane and subsequent fission of the formed vesicle as shown in Fig. 4. Their release was observed to be significantly increased through shear stress (Holme et al, 1997) and the activation of distinct receptors or signaling pathways (Bianco et al, 2005; MacKenzie et al, 2001; Obregon et al, 2006; Pizzirani et al, 2007; Sims et al, 1989). These observations are in line with the fact that calcium is known to induce strong shedding responses (Moskovich \& Fishelson, 2007; Pilzer et al, 2005). An increase in intracellular calcium levels was shown to activate calpain, a calcium-dependent cytosolic protease which mediates Talin degradation (Miyoshi et al, 1996). Loss of the cytoskeletal protein Talin is responsible for destabilization of the membrane cytoskeleton which enables membrane blebbing and MV release (VanWijk et al, 2003). Also inhibition of microtubule assembly by e.g. colchicine, vinblastine or cold temperatures destabilizes the membrane skeleton and favors membrane blebbing (Liepins, 1983). Some insight into the molecular mechanism of MV shedding was contributed recently by Muralidharan-Chari et al who demonstrated a crucial role of the small GTP-binding protein ARF6 in the regulation of MV release by activation of the motor protein Myosin which is important for fission of formed MV (Muralidharan-Chari et al, 2009; Muralidharan-Chari et al, 2010).

MV are characterized by an externalization of phosphatidylserine, a phenomenon also seen on apoptotic bodies. This is due to the fact that both apoptosis and cellular activation are characterized by a disruption of normal membrane asymmetry. In unstimulated, healthy cells membrane asymmetry is mainly achieved by lipid transporter enzymes of the flippase family which flip phosphatidylserine to the cytoplasmic face (VanWijk et al, 2003). In case of cellular activation associated with an increase in intercellular calcium, flippase transporters are inactivated, while outward-directed (e.g. floppase) or bi-directional (e.g. scramblase) lipid transporters are activated (Bevers et al, 1999; Hugel et al, 2005). This finally results in bidirectional phospholipid redistribution and phosphatidylserine externalization. Expression of phosphatidylserine at the external leaflet of the plasma membrane represents a typical recognition signal for phagocytes inducing clearance of the respective cells or vesicles (Fadok et al, 1992). Furthermore, phosphatidylserine and tissue factor which are both present on MV are known to act synergistically in triggering blood coagulation and are therefore viewed as the two main factors being responsible for the procoagulant activity of MV that seems to be 
involved in the pathogenesis of many cardiovascular diseases (Lechner \& Weltermann, 2008; Morel et al, 2006; VanWijk et al, 2003).

There is some evidence that MV function depends on the uptake into their target cell (Kawamoto et al, 2012). In 2009, Cocucci et al suggested that MV uptake could either occur by direct membrane fusion or by endocytosis (Cocucci et al, 2009). Recent studies which demonstrated reduced MV uptake during low temperatures or inhibition of the GTPase dynamin rather point to an endocytosis-mediated uptake (Kawamoto et al, 2012).

\subsubsection{Apoptotic bodies}

From the three EV populations, apoptotic bodies are the least characterized. They are released from apoptotic cells which undergo several steps in their dying process including cell shrinkage, chromatin condensation, nuclear fragmentation, membrane blebbing and finally the breakdown of the cell into apoptotic bodies (Reed, 2000). This is mainly mediated by activity of caspase-3 which belongs to the caspase family of intracellular cysteine proteases and induces reorganization of the cytoskeleton (Shigemura et al, 2001). Apoptotic bodies are mainly cleared by phagocytic cells, especially $\mathrm{M} \phi$, without eliciting an inflammatory response (Henson et al, 2001; Reed, 2000) which is essential for normal tissue homeostasis.

Apoptotic bodies have been described to have a size of 1-4 $\mu \mathrm{m}$ (Hristov et al, 2004). They typically stain positive for Annexin V (Hristov et al, 2004) and show an externalization of phosphatidylserine (Martin et al, 1995) which makes it difficult to discriminate them from MV. However, in contrast to MV, apoptotic bodies specifically stain positive for PI and DAPI (Hristov et al, 2004) resulting from the presence of DNA inside the bodies. Proteomic studies by Turiak et al demonstrated a surprisingly similar protein expression pattern in apoptotic bodies and MV from thymus cells of BALB/c mice (Turiak et al, 2011).

Next to cytoplasm, apoptotic bodies further contain tightly packed cell organelles, in some cases together with nuclear fragments, which could be visualized by electron microscopy (Elmore, 2007). Moreover, RNA was observed to be packed separately from DNA into apoptotic bodies resulting in two different subpopulations of apoptotic bodies: One that is packed with DNA, one that is enriched in RNA (Halicka et al, 2000). Apoptotic bodies also seem to be involved in horizontal transfer of biomolecules since apoptotic bodies of oncogene-transfected cells transferred DNA to $\mathrm{p} 53^{-/-}$mouse embryonic fibroblasts which induced a tumorigenic phenotype of these cells in in vivo experiments (Bergsmedh et al, 2001). Furthermore, apoptotic bodies released from tumor cell lines were able to suppress 
$\mathrm{CD}^{+} \mathrm{T}$ cell responses in vivo (Xie et al, 2009) and induced expression of the growth factor VEGF and the survival factor Bcl-2 in M $\phi$ involved in the clearance of the bodies (Golpon et al, 2004). Taken together, these observations suggest a role of tumor-derived apoptotic bodies in cancer progression.

\subsection{The significance of extracellular vesicles in cancer}

\subsubsection{EV as biomarkers in cancer}

MV and Exo are not only present in in vitro cell culture models, but have been detected in vivo in almost all body fluids including blood, cerebrospinal fluid, breast milk, ascites fluid, semen, urine or saliva (Muralidharan-Chari et al, 2010; Raposo \& Stoorvogel, 2013) suggesting their potential as biomarkers for cancer. A study from Baran et al could already demonstrate elevated levels of EV in peripheral blood of gastric cancer patients, their amount correlating with disease progression and tumor stage (Baran et al, 2010). This was also confirmed for EV present in ascites fluid of ovarian cancer patients (Graves et al, 2004). Further studies revealed that especially the number of procoagulant platelet-derived MV (PMV) carrying tissue factor was elevated in metastatic cancer patients and was associated with venous thromboembolic events and shorter overall survival (Helley et al, 2009; Toth et al, 2008a; Zwicker et al, 2009). In patients with disseminated breast or pancreatic cancer elevated expression of tissue factor and the tumor antigen MUC-1 on circulating MV also correlated with lower survival rates (Tesselaar et al, 2007). Analysis of EV in plasma samples of cancer patients revealed an increased expression of the tumor marker Her-2 (Baran et al, 2010). Profiling of Exo secreted into the urine of patients with bladder cancer compared to healthy controls elucidated an upregulation of 8 proteins in patient samples with five of them being part of oncogenic EGFR signaling (Smalley et al, 2008). Taken together, these studies strongly suggest that EV might be useful as easy accessible targets for early disease detection, monitoring or prognosis establishment in cancer.

\subsubsection{EV as mediators of intercellular communication in cancer}

Several studies have suggested that EV not only serve as potential biomarkers in cancer, but are also actively involved in disease progression. This effect is mainly attributed to their role as mediators of intercellular communication which are able to transport proteins, lipids or nucleic acids from their cell of origin to the recipient cell (Fig. 4) (Cocucci et al, 2009). They 
cannot only influence neighboring cells, but are also transported to distant sites when they are secreted into lymphatic or blood vessels (Peinado et al, 2012). Since EV represent platforms which transport combinations of multiple ligands and receptors, they are considered as more powerful means of intercellular communication compared to single secreted proteins (Cocucci et al, 2009). A major breakthrough was the finding that $\mathrm{EV}$ are often enriched in specific RNA molecules and can transport mRNA as well as miRNA to their recipient cells, where they are transcribed and modulate target gene expression (Mittelbrunn et al, 2011; Ratajczak et al, 2006; Valadi et al, 2007).

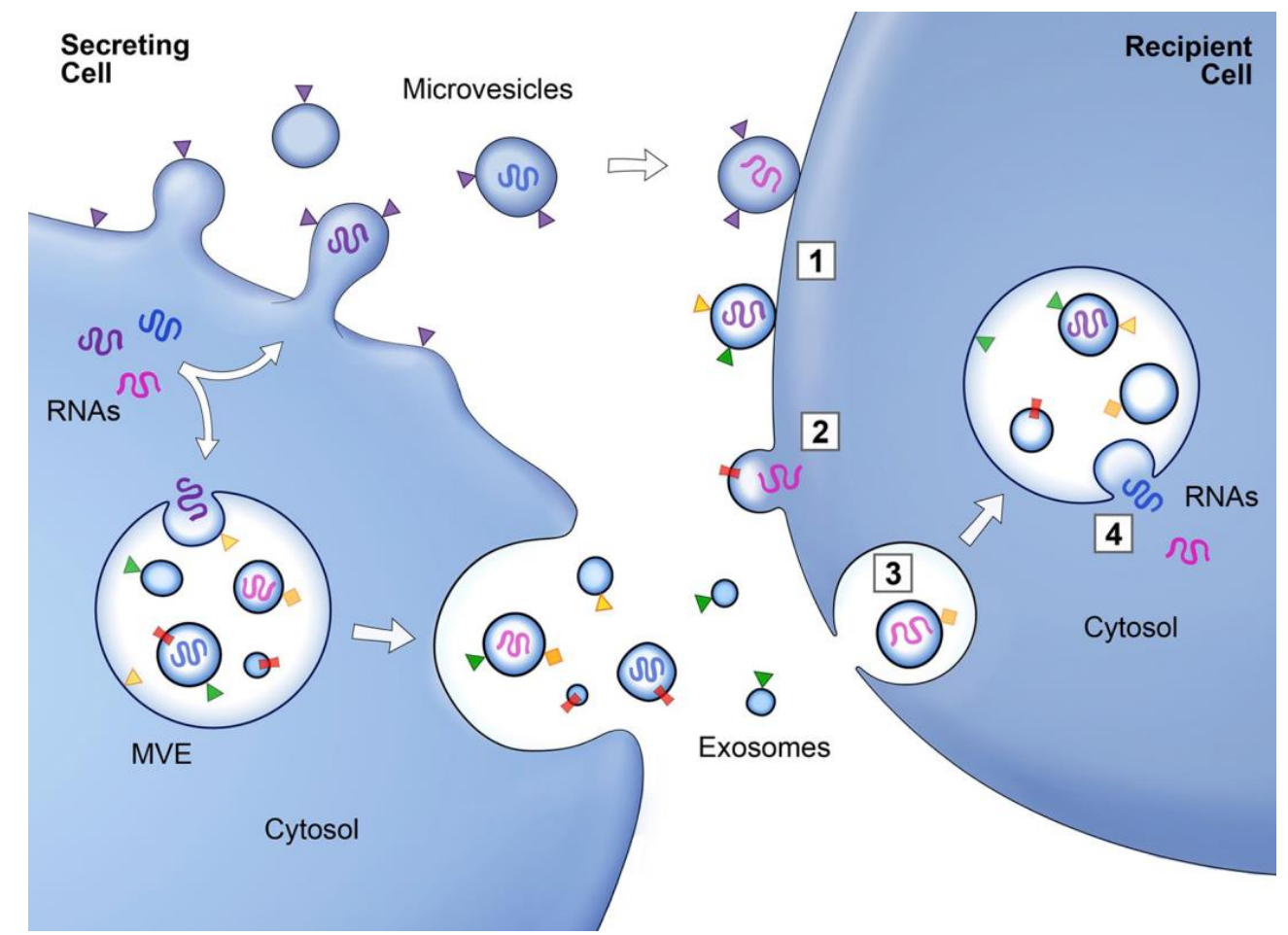

Fig. 4: Horizontal transfer of proteins and nucleic acids by EV

While MV are directly shed from the cellular plasma membrane, Exo are formed by inward budding into the lumen of multivesicular bodies or endosomes (MVB or MVE, respectively). There, they are stored until fusion of MVB with the plasma membrane. During their formation MV and Exo can incorporate cytosolic RNA as well as membrane-associated (triangles and squares) or transmembrane (rectangles) proteins and transport them to their recipient cells. EV can either interact with receptors at the plasma membrane of their target cell (1) or they are taken up by fusion with the plasma membrane (2) or by endocytosis (3). Ingested EV may then release their cargo by fusion with the deliminating membrane of the endocytic compartment (4). Taken from (Raposo \& Stoorvogel, 2013) under a Creative Commons Attribution 3.0 License.

One mechanism how EV contribute to tumor progression is by modulating and preventing anti-tumor immune reactions. Tumor-derived Exo (T-Exo) were shown to suppress NK cell (Liu et al, 2006) and T cell activity (Taylor \& Gercel-Taylor, 2005). Moreover, tumor-derived MV (T-MV) shed by human melanoma or colorectal cancer cells were reported to fuse with monocytes and inhibit their differentiation to antigen-presenting cells, while in parallel 
inducing expression of immunosuppressive cytokines that block activation and function of cytolytic T cells (Valenti et al, 2006). However, there are also studies which show that dendritic cells pulsed with tumor antigens can release Exo which carry functional MHC class I and II molecules and are able to prime tumor-specific cytotoxic T cells (Wolfers et al, 2001; Zitvogel et al, 1998). Apart from their influence on cells of the immune system, T-Exo have been identified as transporters which mediate export of cytostatic drugs out of the tumor cells during chemotherapy (Shedden et al, 2003). Lymphoma cells were shown to release Exo which shield the cells from attacking antibodies in immunochemotherapy approaches (Aung et al, 2011) confirming the role of Exo in the acquisition of chemotherapy resistance.

EV were also shown to be directly involved in tumor progression: On the one hand, MV are known as carriers of MMP and their regulators e.g. EMMPRIN or urokinase-type plasminogen activator ( $\mu \mathrm{PA})$ which help in the degradation of the surrounding ECM and thereby promote tumor invasion (Dolo et al, 1999; Dolo et al, 1998; Muralidharan-Chari et al, 2010). On the other hand, T-Exo from breast cancer cells were observed to directly stimulate tumor cell proliferation in vitro (Koga et al, 2005). Furthermore, EV were found to transfer malignant characteristics from highly malignant to less malignant cells of the same tumor type which led to transformation of the latter and increased the metastatic potential of the cells (AlNedawi et al, 2008; Angelucci et al, 2000; Poste \& Nicolson, 1980). However, studies of direct tumor-tumor interactions via EV are limited. Much more attention has been paid to EV as mediators of tumor-stroma crosstalk. In this context, T-EV have been shown to activate tumor-associated fibroblasts or endothelial cells which, in turn, release pro-invasive factors like VEGF or MMP (Baj-Krzyworzeka et al, 2006; Castellana et al, 2009; Taraboletti et al, 2006) that support tumor invasion and neoangiogenesis. In mouse models of pancreatic cancer and melanoma metastasis, injected T-Exo accumulated in nearby lymph nodes and bone marrow and helped in the creation of a premetastatic niche by transforming benign stroma cells via horizontal transfer of distinct mRNAs and miRNAs (Hood et al, 2011; Peinado et al, 2012; Rana et al, 2013).

\subsection{Aims of the study}

As explained in 1.3.1, $\mathrm{M} \phi$ which get in contact with tumor cells do not acquire a proinflammatory M1 phenotype associated with anti-tumor immunity, but in contrast are reprogrammed by the tumor cells to assist in tumor progression. In line with this, previous results from our group demonstrated that co-culture of human $\mathrm{M} \phi$ with breast cancer cells 
increases tumor invasiveness through induction of Wnt5a in the M $\phi$ (Hagemann et al, 2004; Pukrop et al, 2006). Since in the co-culture system both cell populations are separated by a membrane with a pore diameter of $10 \mu \mathrm{m}$, this suggests that not direct cell-cell interactions, but factors in the supernatant, either soluble or corpuscular, are responsible for intercellular communication. For this reason, it was the aim of this study to further define the role of EV for intercellular communication in human breast cancer progression by answering the following questions:

1) Which EV populations are released by breast cancer cells?

How do they influence the invasiveness of the tumor cells?

Our first goal was to establish a protocol for the reproducible isolation of the two main EV populations, MV and Exo, and identify specific markers for both. Since little is known about the effect of tumor-derived EV (T-EV) on the tumor cells themselves, we then investigated the role of T-EV in tumor-tumor crosstalk and aimed to specify their mode of action.

2) What is the role of T-EV in tumor-M $\phi$ interactions, especially regarding the induction and transport of Wnt5a?

To investigate whether T-EV are responsible for the pro-invasive tumor-M $\phi$ crosstalk, we analyzed if they mediate Wnt5a induction in $\mathrm{M} \phi$. Although Wnt proteins are known to be very hydrophobic and thus limited in their mobility, they participate in short- as well as longrange signaling events (Hardin \& King, 2008). We therefore hypothesized that Wnt proteins are exported on EV and that this process requires the protein Evi which is known to be crucial for Wnt secretion.

3) Are circulating T-EV also present in peripheral blood of metastatic cancer patients?

Which markers can be used to detect and discriminate them from non-tumor cell-derived EV?

Several studies on EV-mediated tumor progression in mouse models have shown the importance of T-EV in premetastatic niche formation in vivo (Hood et al, 2011; Peinado et al, 2012). In order to analyze whether they can also be detected in peripheral blood of cancer patients, we first aimed to characterize T-EV in vitro and then use the defined markers to establish a protocol for the isolation of T-EV in vivo. 


\section{Materials and Methods}

\subsection{Materials}

\subsubsection{Biological material}

\subsubsection{Cell lines}

The cell lines used in this study are listed in Tab. 1.

Tab. 1: Cell lines

\begin{tabular}{|c|c|c|c|c|}
\hline cell line & cell type & characteristics & obtained from & reference \\
\hline hTERT-HME1 & $\begin{array}{l}\text { human mammary } \\
\text { epithelial cell line }\end{array}$ & immortalized with hTERT & $\begin{array}{l}\text { Clontech, Saint- } \\
\text { Germain-en-Laye, } \\
\text { France }\end{array}$ & $\begin{array}{c}\text { (Van der Haegen \& } \\
\text { Shay, 1993) }\end{array}$ \\
\hline L cells & $\begin{array}{c}\text { murine fibroblast } \\
\text { cell line }\end{array}$ & derived from $\mathrm{C} 3 \mathrm{H} / \mathrm{An}$ & $\begin{array}{l}\text { Dr. J. C. Gross, } \\
\text { Heidelberg }\end{array}$ & (Eagle, 1955) \\
\hline Wnt5a-L cells & $\begin{array}{l}\text { murine fibroblast } \\
\text { cell line }\end{array}$ & $\begin{array}{l}\text { derived from } \mathrm{C} 3 \mathrm{H} / \mathrm{An} \text {, stably } \\
\text { transfected to overexpress } \\
\text { hWnt5a }\end{array}$ & $\begin{array}{l}\text { Dr. J. C. Gross, } \\
\text { Heidelberg }\end{array}$ & (Menck et al, 2013) \\
\hline MCF-7 & $\begin{array}{l}\text { human breast } \\
\text { cancer cell line }\end{array}$ & $\mathrm{ER}+, \mathrm{PR}+, \mathrm{Her} 2-$ & DSMZ, Braunschweig & (Soule et al, 1973) \\
\hline MDA-MB231 & $\begin{array}{l}\text { human breast } \\
\text { cancer cell line }\end{array}$ & ER-, PR-, Her2- & ATCC, Wesel & (Cailleau et al, 1974) \\
\hline SK-BR-3 & $\begin{array}{l}\text { human breast } \\
\text { cancer cell line }\end{array}$ & ER-, PR-, Her2+ & ATCC, Wesel & (Trempe, 1976) \\
\hline
\end{tabular}

\subsubsection{Primary cells}

Human platelets were derived from outdated platelet concentrates (expired < 1 day) which were kindly provided by the Department for Transfusion Medicine, University Medical Center Göttingen.

Buffy coats were obtained as well from the Department of Transfusion Medicine, University Medical Center Göttingen, preferably from donors with blood group 0+ or B+, alternatively.

\subsubsection{Patient samples}

Tissues from cerebral metastases were obtained from patients previously diagnosed with primary breast cancer who underwent neurosurgical resection. Peripheral blood for the 
collection of EV was collected from advanced cancer patients diagnosed with multiple metastases. Samples were processed $<30 \mathrm{~min}$ after blood withdrawal to prevent degradation of EV by serum proteases. All of the materials were obtained after informed consent and approved by the University Human Studies Committee of the University of Göttingen (reference numbers: 24/10/05 and 21/3/11).

Human $A B$ serum samples were provided by the Department for Transfusion Medicine, University Medical Center Göttingen. For inactivation of the complement system, samples were heated for $30 \mathrm{~min}$ at $56^{\circ} \mathrm{C}$ followed by centrifugation at $3000 \mathrm{~g}$ for $15 \mathrm{~min}$. Supernatants were pooled and stored at $-20^{\circ} \mathrm{C}$.

\subsubsection{Cell culture media and additives}

All media and additives used for the culture of cell lines and primary cells are listed in Tab. 2.

Tab. 2: Cell culture media and additives

\begin{tabular}{lc}
\hline \multicolumn{1}{c}{ product } & company \\
\hline \hline & Biochrom (Berlin) \\
DMEM medium & Clontech (Saint-Germain-en-Laye, France) \\
Doxycycline & Sigma (München) \\
Fetal calf serum (FCS) & PromoCell (Heidelberg) \\
MEGM medium & Biochrom (Berlin) \\
penicillin/streptomycin (P/S) & Sigma (München) \\
puromycin & Gibco (Darmstadt) \\
RPMI-1640 medium with Phenol Red & PAA (Cölbe) \\
RPMI-1640 medium without Phenol Red & Biochrom (Berlin) \\
Trypsin & \\
\hline
\end{tabular}

\subsubsection{Chemicals, enzymes and recombinant proteins}

All used chemicals, enzymes as well as recombinant proteins were purchased from Sigma (München) unless stated otherwise in Tab. 3 
Tab. 3: Chemicals, enzymes and recombinant proteins

\section{Actinomycin D (Act D)}

Biocoll Separating Solution, density $1,077 \mathrm{~g} / \mathrm{ml}$

phenol/chloroform/isoamyl alcohol (25:24:1)

ECLPrime Western Blot Detection Reagent

ECM gel

EpCAM MicroBeads

$\mathrm{IDC}^{\mathrm{TM}}$ latex particles aldehyde/sulfate, $4 \%(\mathrm{w} / \mathrm{v})$

Laemmli loading buffer, non-reducing, $4 \mathrm{x}$

Laemmli loading buffer Roti ${ }^{\circledR}$-Load 1, reducing, 4x

LPS from Escherichia coli

Percoll $^{\mathrm{TM}}$ Solution (density $1,130 \mathrm{~g} / \mathrm{ml}$ )

Phosphatase inhibitor PhosSTOP, 10x

PNGaseF

Ponceau S

rhDKK1

rhM-CSF

rhWnt5a

SB-203580

SYBR green

\section{Lundbeck Pharmaceuticals Ireland (Dublin, Ireland)}

Biochrom (Berlin)

Roth (Karlsruhe)

GE Healthcare (Freiburg)

R\&D systems (Wiesbaden)

Miltenyi Biotec (BergischGladbach)

Invitrogen (Darmstadt)

bioPLUS (Mol, Belgium)

Roth (Karlsruhe)

Enzo Life Sciences (Lörrach)

GE Healthcare

Roche (Granzach-Wyhlen)

New England Biolabs (Frankfurt a. M.)

Merck (Darmstadt)

R\&D systems (Wiesbaden)

ImmunoTools (Friesoythe)

R\&D systems (Wiesbaden)

Calbiochem (Darmstadt)

Roche (Mannheim)

\subsubsection{Antibodies}

The antibodies used in this study are listed in Tab. 4 .

Tab. 4: Antibodies

\begin{tabular}{ccccc}
\hline target & host species & label & application & company \\
\hline \hline CD11c & mouse & PE & FC $(5 \mu 1)$ & \#IM1760, Beckman Coulter (Krefeld) \\
CD14 & mouse & FITC & FC $(5 \mu 1)$ & \#325603, BioLegend (Fell) \\
CD45 & mouse & FITC & FC $(5 \mu 1)$ & \#7782, Beckman Coulter (Krefeld) \\
CD62P & mouse & FITC & FC $(5 \mu 1)$ & \#555523, BD (Heidelberg) \\
CD62E & mouse & PE & FC $(4 \mu 1)$ & \#336008, BioLegend (Fell) \\
CD63 & mouse & - & FC $(1 \mu 1)$ & \#556019, BD (Heidelberg) \\
CD235a & mouse & PE & FC $(5 \mu 1)$ & \#A07792, Beckman Coulter (Krefeld) \\
c-jun & rabbit & - & WB $(1: 1000)$ & \#9165, cell signaling (Frankfurt a. M.) \\
EGFR & mouse & PE & FC $(1 \mu 1)$ & \#352903, BioLegend (Fell) \\
EMMPRIN & rabbit & - & WB $(1: 1000)$ & $\#$ \#sc-13976, santa cruz (Heidelberg) \\
& & & FC $(5 \mu l)$ & \\
\hline
\end{tabular}




\begin{tabular}{|c|c|c|c|c|}
\hline target & host species & label & application & company \\
\hline EMMPRIN & mouse & $\begin{array}{c}\text { Alexa } \\
\text { Fluor } 488\end{array}$ & $\mathrm{FC}(0,5 \mu 1)$ & \#306207, BioLegend (Fell) \\
\hline EpCAM & mouse & APC & $\mathrm{FC}(5 \mu \mathrm{l})$ & \#324207, BioLegend (Fell) \\
\hline Evi/WLS & rabbit & - & WB $(1: 500)$ & \#MABS87, Millipore (Darmstadt) \\
\hline Flotillin-2 & mouse & - & WB $(1: 1000)$ & \#610383, BD (Heidelberg) \\
\hline Her2/neu (erb-B2) & rabbit & - & $\begin{array}{l}\text { WB }(1: 1000) \\
\text { FC }(1 \mu \mathrm{l})\end{array}$ & \#2165, cell signaling (Frankfurt a. M.) \\
\hline \multirow[t]{4}{*}{ IgG1 } & mouse & $\begin{array}{c}\text { Alexa } \\
\text { Fluor } 488\end{array}$ & $\mathrm{FC}(1 \mu \mathrm{l})$ & \#400132, BioLegend (Fell) \\
\hline & mouse & FITC & $\mathrm{FC}(5 \mu \mathrm{l})$ & \#345815, BD (Heidelberg) \\
\hline & mouse & $\mathrm{PE}$ & $\mathrm{FC}(5 \mu \mathrm{l})$ & \#A07796, Beckman Coulter (Krefeld) \\
\hline & mouse & $\mathrm{PE}$ & $\mathrm{FC}(1 / 0,5 \mu \mathrm{l})$ & \#400114, BioLegend (Fell) \\
\hline $\operatorname{IgG} 2 b$ & mouse & APC & $\mathrm{FC}(0,156 \mu \mathrm{l})$ & \#400322, BioLegend (Fell) \\
\hline mouse & Goat & $\begin{array}{l}\text { HRP } \\
\text { FITC }\end{array}$ & $\begin{array}{l}\text { WB }(1: 6000) \\
\text { FC }(0,5 \mu \mathrm{l})\end{array}$ & $\begin{array}{l}\text { \#sc-2005, santa cruz (Heidelberg) } \\
\text { \#sc-2010, santa cruz (Heidelberg) }\end{array}$ \\
\hline MUC1 & mouse & $\mathrm{PE}$ & $\mathrm{FC}(1 \mu \mathrm{l})$ & \#355603, BioLegend (Fell) \\
\hline p38 total & rabbit & - & WB (1:1000) & \#9212, cell signaling (Frankfurt a. M.) \\
\hline P-c-jun & rabbit & - & WB (1:1000) & \#9261, cell signaling (Frankfurt a. M.) \\
\hline P-p38 & rabbit & - & WB $(1: 1000)$ & \#9211, cell signaling (Frankfurt a. M.) \\
\hline rabbit & goat & $\begin{array}{l}\text { HRP } \\
\text { FITC }\end{array}$ & $\begin{array}{l}\text { WB }(1: 6000) \\
\text { FC }(0,5 \mu 1)\end{array}$ & $\begin{array}{c}\text { \#sc-2004, santa cruz (Heidelberg) } \\
\text { \#406403, BioLegend (Fell) }\end{array}$ \\
\hline rat & goat & HRP & WB (1:6000) & \#sc-2006, santa cruz (Heidelberg) \\
\hline RUNX1 & rabbit & - & WB (1:500) & \#2593 cell signaling (Frankfurt a. M.) \\
\hline Tubulin & mouse & - & WB (1:5000) & \#05-829, Millipore (Darmstadt) \\
\hline Tsg101 & mouse & - & WB $(1: 1000)$ & \#7964, santacruz (Heidelberg) \\
\hline Wnt5a & rat & - & WB (1:2000) & \#MAB645, R\&D systems(Wiesbaden) \\
\hline
\end{tabular}

\subsubsection{Oligonucleotides, plasmids and shRNA constructs}

Tab. 5 depicts all oligonucleotides, Tab. 6 all plasmids used for this study. The shRNA constructs for the stable knockdown of Evi/WLS were obtained from Sigma and were kindly provided by Dr. J. C. Gross (German Cancer Center, Heidelberg). The ns ctl sequence is 5'CCCGTGTAAATATGTACATTT-3', the EVI targeting sequence is 5'GATCTACAAGTTGACCCGCAA-3'.

The shRNA constructs for the stable knockdown of EMMPRIN were purchased from Thermo Scientific. While the ns ctl sequence is proprietary, the EMMPRIN targeting sequence is TCCAAGTTCTCACCTCTTA (Oligo ID: V3THS_412786). 
Tab. 5: Oligonucleotides

name

sequence $\left(5^{6}-3^{6}\right)$

eGFP_fw120

eGFP_rv120

hsEMMPRIN12_fw82

hsEMMPRIN12_rv189

hscjun_fw77

hscjun_rv77

hsEVI_fw84

hsEVI_rv84

hsGNB2L1_fw84

hsGNB2L1_rv84

hsHPRT1_fw89

hsHPRT1_rv89

hsIL-1B_fw131

hsIL-1ß_rv72

hsIL10_fw57

hsIL10_rv57

hsMMP2_fw130

hsMMP2_rv130

hsMMP7_fw126

hsMMP7_rv126

hsMMP9_fw100

hsMMP9_rv61

hsMT1-MMP_fw134

hsMT1-MMP_rv134

hsMT2-MMP_fw71

hsMT2-MMP_rv71

hsTNFa_fw78

hsTNFa_rv176

hsVEGF-A_fw125

hsVEGF-A_rv125

hsWnt5a Ex4 fw

hsWnt5a Ex5 rv

mmEVI_fw69

mmEVI_rv69

mmGAPDH_fw146

mmGAPDH_rv146
CAAGATCCGCCACAACATCG

GACTGGGTGCTCAGGTAGTG

GCGAGGAATAGGAATCATGG

TCTACGGTAGTGAAGACTGTG

CCTTGAAAGCTCAGAACTC

CTTTCTGTTTAAGCTGTGCC

ACTACAGACATTGGAACAGAG

AGCATAGAAACAGGAAGTAGAG

AACCCTATCATCGTCTCCT

CAATGTGGTTGGTCTTCAG

TATGCTGAGGATTTGGAAAGG

CATCTCCTTCATCACATCTCG

TACGATCACTGAACTGCAC

CAAAGGACATGGAGAACAC

CTTGCTGGAGGACTTTAAGG

CTGGATCATCTCAGACAAGG

ACCTACACCAAGAACTTCC

CAGATCTCAGGAGTGACAG

CAGATGTTGCAGAATACTCAC

CACTGTAATATGCGGTAAGTC

ACCTGAGAACCAATCTCAC

GTAACCATAGCGGTACAGG

GAAGGATGGCAAATTCGTC

GAGCAGCATCAATCTTGTC

GAGATGTTCGTGTTCAAGG

CATGGGATAGTTGTCCAGG

TCTCTAATCAGCCCTCTGG

CTACAACATGGGCTACAGG

CTGAGGAGTCCAACATCAC

GTCTTGCTCTATCTTTCTTTGG

AGGGCTCCTACGAGAGTGCT

GACACCCCATGGCACTTG

GGAGATAAAGGACATTCGGT

CATAGCAAACCATACCTTAGTG

CATCTTGGGCTACACTGAG

CTGTAGCCGTATTCATTGTC 


\begin{tabular}{ll}
\hline \multicolumn{1}{c}{ name } & sequence $\mathbf{( 5}^{\mathbf{6}} \mathbf{- \mathbf { 3 } ^ { \mathbf { 6 } } )}$ \\
\hline \hline mmTBP_fw133 & \\
mmTBP_rv133 & TTCCCAGCTAAGTTCTTAGAC \\
mmWnt5a_fw72 & CCAGGAAATAATTCTGGCTC \\
mmWnt5a_rv72 & TTACACAACAATGAAGCAGG \\
xWnt5a_fw130 & ACACTCCATGACACTTACAG \\
xWnt5a_rv130 & GTTTGCCAAGGAGTTTGTG \\
& CGGCCAAGGTACTTACTG \\
\hline
\end{tabular}

Tab. 6: Plasmids

\begin{tabular}{ccc}
\hline plasmid & characterstics/genotype & reference \\
\hline \hline pCMV $\triangle$ R8.91 & packaging plasmid & \\
pCS2+-xWnt5a-eGFP & encodes a Xenopus xWnt5a-eGFP fusion protein & $\begin{array}{r}\text { Addgene (Cambridge, USA) } \\
\text { PD. Dietmar Gradl, Karlsruhe Institute } \\
\text { of Technology, unpublished }\end{array}$ \\
pVSG-G & packaging plasmid & Addgene (Cambridge, USA) \\
\hline
\end{tabular}

\subsubsection{Commercial kits and standards}

All commercial kits and standards for agarose or protein gels are listed in Tab. 7.

Tab. 7: Commercial kits and standards

product company

$\mathrm{D}_{\mathrm{c}}$ protein assay

DNA ladder $100 \mathrm{~kb}$

High Pure RNA Isolation kit

iScript cDNA synthesis kit

LD columns for MACS

Legend $\operatorname{Max}^{\mathrm{TM}}$ ELISA kits for IL-1ß, IL-10, IL-12

LS columns for MACS

Nanofectin Kit

PKH26 Red Fluorescent Cell Linker Kit

Precision Plus Dual-Color protein standard

Refraction-2D ${ }^{\mathrm{TM}}$ Labeling Kit

$$
\text { Bio-Rad (München) }
$$

Fermentas (St. Leon-Rot)

Roche (Grenzach-Wyhlen)

Bio-Rad (München)

Miltenyi Biotec (Bergisch-Gladbach)

BioLegend (Fell)

Miltenyi Biotec (Bergisch-Gladbach)

$$
\text { PAA (Cölbe) }
$$

Sigma (München)

Bio-Rad (München)

DyeAGNOSTICS (Halle) 


\subsubsection{Equipment}

All lab equipment used for the experiments is listed in Tab. 8 .

Tab. 8: Equipment

\section{product}

\section{company}

autoclave Varioklav

Axiovert $200 \mathrm{M}$ fluorescence microscope

CCD camera for transilluminator

centrifuge Multifuge 3 L-R Heraeus

$\mathrm{CO}_{2}$ incubator $\mathrm{CB} 150$

FACS Canto II flow cytometer

FEP Teflon-coated cell culture bags, small/large

MACS separator MACSVario

Magnetic stirrer REO basic C

MilliQ water purification system

NanoDrop ND-1000 spectrophotometer

Neubauer Improved cell counting chamber

pH meter 761 Calimatic

Pipetboy

Photometer infinite F50

refrigerated microfuge SIGMA $1-15 \mathrm{~K}$

rotor $\mathrm{Sw} 32 \mathrm{Ti}$ for ultracentrifugation $(6 \mathrm{x} 38,5 \mathrm{ml})$

rotor $\mathrm{Sw} 32.1 \mathrm{Ti}$ for ultracentrifugation $(6 \times 17 \mathrm{ml})$

rotor TLA-120.2 for ultracentrifugation $(10 \times 1,5 \mathrm{ml})$

Thermal cycler T3000

thermomixer 5437

ultracentrifuge Optima XPN-80

ultracentrifuge TL-100

UV transilluminator TI3

vortex shaker Genius 3

Water bath
Thermo Scientific (Bonn)

$$
\begin{gathered}
\text { Zeiss (Jena) } \\
\text { Intas (Göttingen) }
\end{gathered}
$$

Thermo Scientific (Bonn)

Binder (Tuttlingen)

BD (Heidelberg)

CellGenix (Freiburg)

Miltenyi Biotec (Bergisch-Gladbach)

IKA Lab equipment (Staufen)

Millipore (Schwalbach)

Peqlab (Erlangen)

LO Laboroptik (Friedrichsdorf)

Knick Elektronische Messgeräte (Berlin)

Integra biosciences (Femwald)

Tecan (Crailsheim)

Sigma Laborzentrifugen (Osterode am Harz)

Beckman Coulter (Krefeld)

Beckman Coulter (Krefeld)

Beckman Coulter (Krefeld)

Biometra (Göttingen)

Eppendorf (Hamburg)

Beckman Coulter (Krefeld)

Beckman Coulter (Krefeld)

Biometra (Göttingen)

IKA Lab equipment (Staufen)

Köttermann (Uetze,Hänigsen) 


\subsection{Methods}

\subsubsection{Cell culture methods}

\subsubsection{Maintenance of cells}

Cells were grown at $37^{\circ} \mathrm{C}$ and $5 \% \mathrm{CO}_{2}$ in a humidified incubator. To passage the cells, they were washed once with PBS, detached by incubation with 1-2 ml Trypsin for up to $15 \mathrm{~min}$ and splitted in a ratio of 1:10-1:40. In case of hTERT-HME1, the cells were scraped carefully from the bottom of the cell culture flask and splitted in a ratio of 1:5. All cell lines were routinely tested for contamination with Mycoplasma. To maintain cells over a longer period of time, they were frozen in DMSO $+90 \% \mathrm{FCS}$ and stored at $-150^{\circ} \mathrm{C}$.

\subsubsection{Generation of stable knockdown cell lines}

Cell lines with stable knockdown of EMMPRIN or Evi expression were generated through shRNA-mediated gene silencing. For production of lentiviral particles, HEK293T cells were co-transfected through calcium phosphate precipitation with the packaging plasmids $\mathrm{pVSG-G}$ and pCMV $\triangle \mathrm{R} 8.91$ as well as the shRNA construct of interest (see 2.1.5). The viral particles which are released from the cells into the supernatant were concentrated using a lentiviral enrichment reagent (MoBiTec) according to the manufacturer's instructions and were used for subsequent transduction of SK-BR-3 or Wnt5a-L cells, respectively. Lentiviral vectors can infect a broad range of cells, including dividing and even non-dividing cells, and integrate into the genome of their target cells, which allows stable expression of the gene of interest. Selection of transfected cells was achieved by adding $2 \mu \mathrm{g} / \mathrm{mL}$ (for SK-BR-3) or $15 \mu \mathrm{g} / \mathrm{ml}$ (for Wnt5a-L cells) puromycin (Sigma Aldrich) to the culture medium. Since shEMMPRIN cells were transfected with a pTRIPZ vector that comprises a "Tet-On" system, gene knockdown had to be accomplished by addition of the tetracycline doxycycline $(1 \mu \mathrm{g} / \mathrm{ml}$, Clontech) for $72 \mathrm{~h}$.

\subsubsection{Transient transfection with $x W n t 5 a$}

In order to investigate Wnt5a expression in SK-BR-3 shEvi and corresponding non-sense control cells, they were transiently transfected with a plasmid encoding a Xenopus Xwnt5aeGFP fusion protein. The transfection of the cell lines was performed by Dr. Eva Rietkötter (University Medical Center Göttingen). Briefly, $2 \cdot 10^{6}$ cells were seeded in T75 cell culture flasks and allowed to adhere overnight. Transient transfection was performed with the 
Nanofectin Kit (PAA) according to the manufacturer's instructions. This method is based on nanoparticles which contain DNA-binding positively charged polymers and mediate transfer of the DNA into the target cells. Each cell line was transfected with $16 \mu \mathrm{g}$ plasmid in a DNA/Nanofectin ratio of 3. Twenty-four hours after transfection cells were trypsinized and used for protein (see 2.2.3.1) or RNA (see 2.2.5.2) isolation.

\subsubsection{Isolation of human monocytes from Buffy Coats}

Human monocytes were isolated from Buffy Coats with a double density gradient centrifugation protocol as described in (Menck et al, 2014a). A Buffy Coat is the interphase between plasma and erythrocytes which remains after density gradient centrifugation of blood samples and contains most of the platelets and leukocytes. Briefly, $40 \mathrm{ml}$ of each Buffy Coat were layered carefully on top of a Ficoll gradient (15 ml Biocoll separating solution, Biochrom) and centrifuged at $400 \mathrm{~g}$ for $20 \mathrm{~min}$ (without brake). Due to their higher density, granulocytes and remaining erythrocytes pellet on the bottom of the gradient, while mononuclear cells as well as platelets are contained in the whitish interphase between plasma and Ficoll. The interphase was collected and washed twice with PBS +1 mM EDTA at $300 \mathrm{~g}$ for $10 \mathrm{~min}$ (without brake) to deplete remaining platelets. All leukocyte pellets from one Buffy Coat were pooled in $20 \mathrm{ml}$ RPMI-1640 medium without phenol red (PAA) and layered carefully on top of $25 \mathrm{ml}$ of a $46 \%$ iso-osmotic Percoll gradient. The gradient was centrifuged at $550 \mathrm{~g}$ for $30 \mathrm{~min}$ (without brake). The colloidal silica particles coated with polyvinylpyrrolidone which are contained in the Percoll solution lead to the separation of lymphocytes and monocytes (Feige, $\mathrm{U}$ et al, 1982, J Immunol Methods) which results in the sedimentation of the lymphocytes at the bottom of the tube while the monocytes are localized in the whitish interphase. The interphase was collected, resuspended in $20 \mathrm{ml} P B S+1 \mathrm{mM}$ EDTA and centrifuged again at $400 \mathrm{~g}$ for $10 \mathrm{~min}$. The monocyte pellet was resuspended in $20 \mathrm{ml}$ RPMI-1640 $+10 \%$ FCS and the cell count was determined with a Neubauer Cell Counting Chamber (LO Laboroptik).

$\underline{46 \% \text { iso-osmotic Percoll gradient (for two Buffy Coats): }}$

- 23,13 $\mathrm{ml}$ Percoll ${ }^{\mathrm{TM}}$ solution (GE Healthcare)

- $1,87 \mathrm{ml}$ 10x PBS (w/o Ca and Mg, Invitrogen)

Transfer $23 \mathrm{ml}$ of this solution to a new tube and add $27 \mathrm{ml}$ RPMI+10\%FCS with Phenol Red 


\subsubsection{Differentiation of human monocytes to macrophages}

The isolated monocytes were subsequently differentiated to macrophages $(M \phi)$ in the presence of low concentrations of Macrophage colony-stimulating factor (M-CSF/CSF1, ImmunoTools) in fluorinated ethylene propylene (FEP) Teflon-coated cell culture bags (CellGenix) which allows the detachment of the cells after the differentiation period.

$1,0-1,5 \cdot 10^{8}$ monocytes from one Buffy Coat were seeded in large, 3,0-5,0 $\cdot 10^{7}$ monocytes in small FEP Teflon-coated cell culture bags. For each bag, culture medium containing $2 \%$ human AB-serum (see 2.1.1.3), $1 \%$ penicillin/streptomycin and 2,5 ng/ml M-CSF (total volume: $180 \mathrm{ml}$ for large bags, $30 \mathrm{ml}$ for small bags) was prepared and mixed with the respective number of isolated monocytes. The cells were differentiated for $6-7$ days at $37^{\circ} \mathrm{C}$ and $5 \% \mathrm{CO}_{2}$. To harvest the cells, the bags were incubated for $1 \mathrm{~h}$ on ice and subsequently pulled with minimal pressure 10 times over the edge of a desk or board. The cell suspension was removed from the bag and centrifuged at $400 \mathrm{~g}$ for $10 \mathrm{~min}$ to pellet the $\mathrm{M} \phi$. Cells were counted in a Neubauer Cell Counting Chamber and seeded according to the experimental setup. After adherence of the cells, $\mathrm{M} \phi$ were washed twice before they were used for further experiments.

\subsubsection{Generation of activated macrophages by stimulation with tumor cell supernatant}

Human $\mathrm{M} \phi$ show a specific activated phenotype when they get in contact with tumor cells. To simulate this activation without having to perform expensive and time-consuming co-culture experiments, $\mathrm{M} \phi$ were seeded in 6-well-plates at a concentration of $2 \cdot 10^{6}$ cells per well. The wells had been previously coated with $80 \mu \mathrm{l} \mathrm{ECM} \mathrm{gel} \mathrm{(R \& D} \mathrm{systems)} \mathrm{diluted} \mathrm{1:16} \mathrm{in} \mathrm{RPMI-}$ 1640. After adherence of the cells, they were washed twice with PBS and then stimulated with $2 \mathrm{ml}$ tumor cell supernatant per well for $24 \mathrm{~h}$.

The tumor cell supernatant, which should still contain all of the MV and Exo present, was generated by culturing MCF-7 cells in T175 cell culture flasks (confluence of $>70 \%$ ) for $48 \mathrm{~h}$ in RPMI-1640 $+10 \%$ particle-free FCS. The supernatant was collected and centrifuged for $5 \mathrm{~min}$ at $750 \mathrm{~g}$ to remove cells and debris. 


\subsubsection{Assessment of cell vitality and proliferation}

\subsection{MTT assay}

To measure cell viability upon treatment with different inhibitors, the MTT assay was used (Mosmann, 1983). The method is based on the conversion of the water-soluble, yellow tetrazolium salt 3-(4,5-Dimethylthiazol-2-yl)-2,5-diphenyltetrazoliumbromid (MTT, Sigma) to an insoluble, purple formazan. This reduction is mediated by $\mathrm{NAD}(\mathrm{P}) \mathrm{H}$-dependent enzymes in the endoplasmic reticulum (Berridge et al, 1996) and is thus impaired in apoptotic or necrotic cells with an altered cell metabolism.

For the MTT assay, 7,5 $\cdot 10^{4}$ MCF-7, $4 \cdot 10^{4}$ SK-BR-3, $1 \cdot 10^{4}\left(\mathrm{Wnt5a}\right.$-) L cells or $1,5 \cdot 10^{5}$ human M $\phi$ per well were seeded in duplicate in a 24-well-plate and incubated with the respective inhibitors at the indicated concentrations and time periods. Subsequently, the cells were incubated with $500 \mu \mathrm{l}$ culture medium $+10 \%$ MTT (stock solution: $5 \mathrm{mg} / \mathrm{ml}$ ) for $4 \mathrm{~h}$ at $37^{\circ} \mathrm{C}$ and $5 \% \mathrm{CO}_{2}$. The medium was aspired and cells lysed in $500 \mu 15 \%$ formic acid $+63 \%$ isopropanol $+32 \%$ DMSO. The extinction at $540 \mathrm{~nm}$ was measured in triplicate in a photometer and related to the extinction of the untreated control.

\subsubsection{2 xCELLigence}

Analysis with the xCELLigence system (RTCA DP Analyzer, Roche), an electronic impedance-based cell sensing measurement system, was used as an additional method to measure changes in cell viability and proliferation. For the experiments, $1 \cdot 10^{4} \mathrm{MCF}-7$ cells were seeded per well in E16 plates (Roche) and stimulated with different concentrations of filipin III (Sigma) or dansylcadaverine (Santa Cruz) for $72 \mathrm{~h}$. Since the area on the bottom of the wells is covered with a gold microelectrode, changes in electrical impedance can be measured when the cells attach and spread on the electrode surface. Thus, cell growth and spreading result in increased impedance, while detachment or rounding up of cells cause a reduction. The measured impedance is displayed as so-called cell index.

\subsubsection{Cell invasion assay in a modified Boyden chamber}

Cell invasion was quantified in a modified Boyden chamber assay (see Fig. 5) as described previously (Hagemann et al, 2004). Briefly, the lower wells of the chamber were filled with RPMI-1640 $+10 \%$ (or $1 \%$ for co-culture experiments) particle-free FCS and sealed with a polycarbonate membrane (pore diameter: $10 \mu \mathrm{m}$, Pieper Filter) which was coated with ECM gel (R\&D systems) diluted 1:4 in RPMI-1640. This setting is thought to resemble the in vivo situation in the tissue where the tumor is forming. 


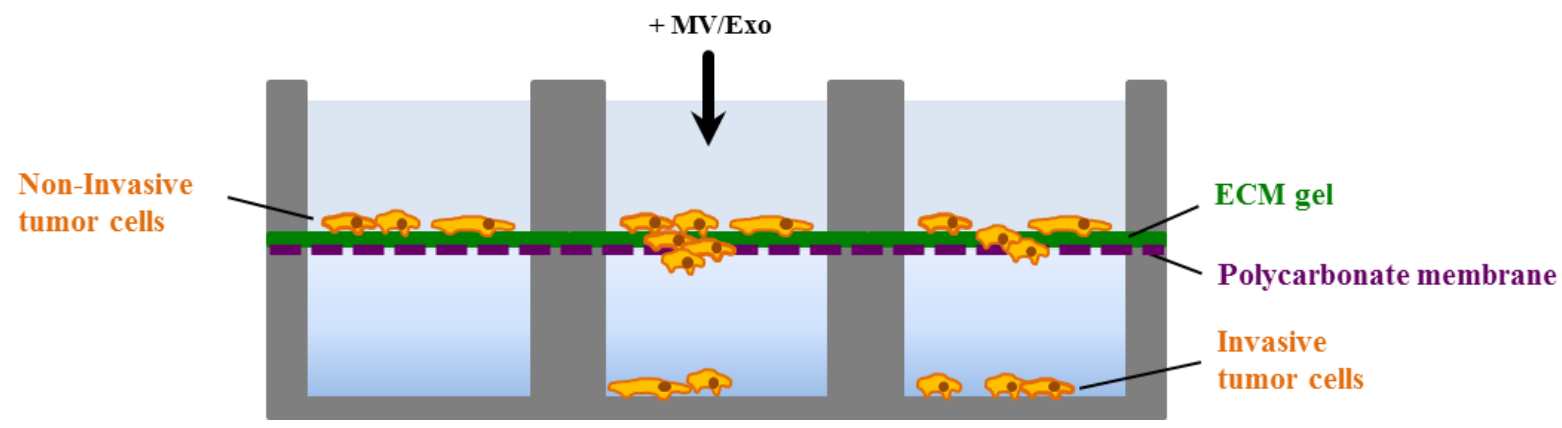

Fig. 5: Schematic representation of the modified Boyden chamber assay.

The cells are seeded in the upper wells of the chamber and stimulated with EV or other factors. After $96 \mathrm{~h}$ the number of invasive cells which had successfully degraded the ECM gel and invaded through the membrane into the lower wells was counted and related to the unstimulated control.

$1 \cdot 10^{5}$ cells were seeded in the upper wells and stimulated in triplicate with MV, Exo or other factors as indicated. The chamber was incubated for $96 \mathrm{~h}$ at $37^{\circ} \mathrm{C}, 5 \% \mathrm{CO}_{2}$ and subsequently the number of invasive cells in the lower wells was determined with a Neubauer Counting Chamber and related to the unstimulated control. Because of the toxicity of the inhibitor, experiments with dynasore were already stopped after $48 \mathrm{~h}$ of stimulation.

For quantification of tumor cell invasion in co-culture with human $\mathrm{M} \phi, 2 \cdot 10^{5} \mathrm{M} \phi$ were seeded in co-culture inserts (Millipore). $1 \mathrm{~h}$ after seeding of the tumor cells in the upper wells, $\mathrm{M} \phi$ inserts were added to the respective wells.

\subsubsection{Extracellular vesicle techniques}

\subsubsection{Isolation of microvesicles (MV) and exosomes (Exo)}

\subsection{Cell culture supernatants}

To isolate EV from adherent cells, the latter were cultured in seven T175 cell culture flasks up to $60-80 \%$ confluence, then washed twice with PBS and incubated for $48 \mathrm{~h}$ in culture medium supplemented with $10 \%$ particle-free FCS. Human M $\phi$ were seeded in $10 \mathrm{~cm}$ cell culture Petri dishes (Sarstedt) in a concentration of $3,5 \cdot 10^{6}$ cells per dish and incubated for $24 \mathrm{~h}$ in RPMI $+1 \%$ particle-free FCS. The particle-free FCS was generated by pelleting endogenous EV by ultracentrifugation over night at $100.000 \mathrm{~g}$ and $4{ }^{\circ} \mathrm{C}$.

Cell culture supernatants were collected and centrifuged at $750 \mathrm{~g}$ for $5 \mathrm{~min}$, followed by $1.500 \mathrm{~g}$ for $15 \mathrm{~min}$ to pellet cells and debris. MV were pelleted by ultracentrifugation in a $\mathrm{Sw} 32 \mathrm{Ti}$ rotor at $14.000 \mathrm{~g}$ and $4^{\circ} \mathrm{C}$ for $35 \mathrm{~min}$. The pellet was washed once in PBS and then 
resuspended in PBS or RIPA lysis buffer. For the isolation of Exo, the supernatant of the $14.000 \mathrm{~g}$ centrifugation step was filtered $(0,22 \mu \mathrm{m}$ filter, Sarstedt) and ultracentrifuged at $100.000 \mathrm{~g}$ and $4^{\circ} \mathrm{C}$ for $2 \mathrm{~h}$. The Exo pellet was washed once in PBS in a TLA-120.2 rotor and then resuspended in PBS or RIPA lysis buffer. MV and Exo counts were routinely determined as described in 2.2.3.2.

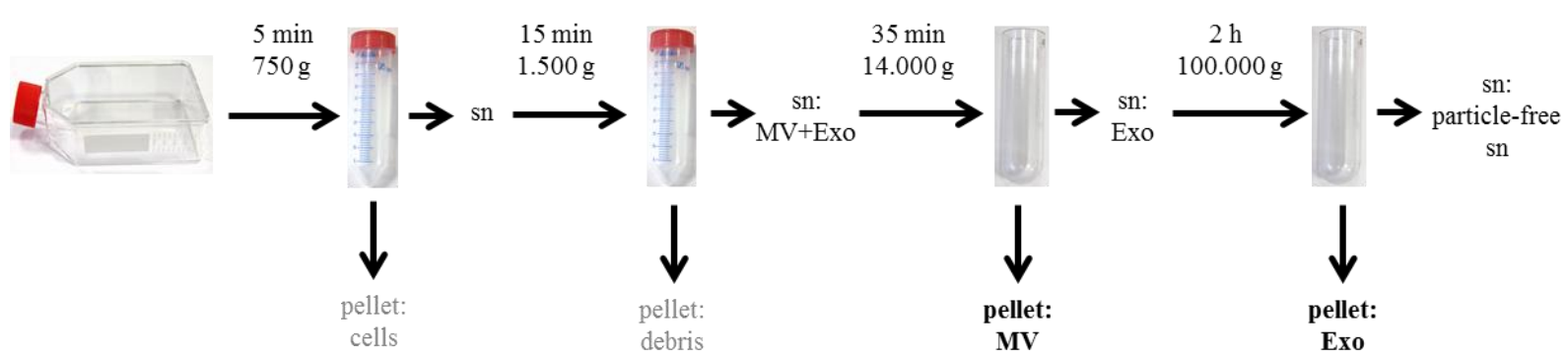

Fig. 6: Schematic representation of the $\mathrm{EV}$ isolation protocol

EV were isolated from cell culture supernatants by a series of differential (ultra)centrifugation steps as indicated. The particle-free supernatant (sn) after precipitation of Exo was employed as negative control for the experiments.

\subsection{Platelet concentrates}

P-MV were isolated according to the protocol in 2.2.2.1.1 from outdated platelet concentrates (outdated <2 days) which were provided by the Department of Transfusion Medicine, University Medical Center Göttingen.

\subsection{Peripheral blood from cancer patients and controls}

For the isolation of total MV and Exo from the peripheral blood of cancer patients, blood samples were collected from cancer patients with metastatic disease who did not receive chemotherapy at the time of sample acquisition. This setting should guarantee the maximal count of tumor-derived EV in the blood, while preventing the presence of apoptotic bodies or activated EV from dying cells which might be present during administration of chemotherapy. As negative control, blood was also collected from matched tumor-free patients or healthy individuals. Samples were collected through a 19 gauge butterfly needle and dispended into tubes containing either EDTA (1,6 mg / ml blood, Sarstedt) or lithium-heparin (16 I.E. / ml blood, Sarstedt). Serum was obtained by centrifugation at $1.200 \mathrm{~g}$ for $15 \mathrm{~min}$ and separation from the blood cells further supported through the application of a serum filter (Seraplas ${ }^{\circledR}$, Sarstedt). The serum was centrifuged at $1.500 \mathrm{~g}$ for $15 \mathrm{~min}$ to pellet debris and then stored at $20^{\circ} \mathrm{C}$ or directly subjected to EV isolation as described in 2.2.2.1.1. The isolation of tumorderived EV from cancer patients by magnetic-activated cell sorting (MACS) is further described in 2.2.4.3. 


\subsubsection{Sucrose gradient ultracentrifugation}

For sucrose gradient preparations, $\mathrm{T}-\mathrm{MV}_{\mathrm{S}}$ and $\mathrm{T}-\mathrm{Exo}_{\mathrm{S}}$ were isolated according to chapter 2.2.2.1.1 and pellets were resuspended in $500 \mu \mathrm{l} 0,25 \mathrm{M}$ sucrose. The vesicles were then applied onto a sucrose step gradient which was prepared by layering decreasing sucrose density solutions ( $2 \mathrm{ml}$ each) upon one another as listed in Tab. 9. The gradient was centrifuged in a Sw 32.1 Ti rotor for $16 \mathrm{~h}$ at $100.000 \mathrm{~g}$ and $4{ }^{\circ} \mathrm{C}$.

Tab. 9: Sucrose step gradient

\begin{tabular}{cccc}
\hline $\mathbf{2 , 5}$ M sucrose $[\boldsymbol{\mu l}]$ & HEPES buffer $[\boldsymbol{\mu l}]$ & molarity $[\mathbf{M}]$ & density $\left[\mathbf{g ~ m l}^{\mathbf{- 1}}\right.$ ] \\
\hline \hline & 4500 & 0,25 & 1,03 \\
1500 & 3500 & 0,75 & 1,06 \\
2000 & 3000 & 1,00 & 1,09 \\
2500 & 2500 & 1,25 & 1,16 \\
3000 & 2000 & 1,50 & 1,19 \\
3500 & 1500 & 1,75 & 1,22 \\
4000 & 1000 & 2,00 & 1,25 \\
4500 & 500 & 2,25 & 1,28 \\
\hline
\end{tabular}

Eight fractions with $2 \mathrm{ml}$ each were collected and proteins were precipitated by adding $18 \mathrm{ml}$ ice-cold acetone and incubating overnight at $-20^{\circ} \mathrm{C}$. Samples were centrifuged for $30 \mathrm{~min}$ at

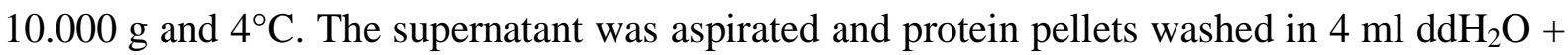
$16 \mathrm{ml}$ acetone $\left(-20^{\circ} \mathrm{C}\right)$ until the remaining sucrose had been washed out. Pellets were dried for $1 \mathrm{~h}$ at room temperature and the protein was resuspended in $80 \mu \mathrm{l}$ RIPA lysis buffer for Western Blot analysis.

HEPES buffer $(20 \mathrm{mM})$ :

- 4,766 g HEPES

- Ad $11 \mathrm{ddH}_{2} \mathrm{O}$ and adjust $\mathrm{pH}$ at 7,4

\section{$\underline{2,5 \mathrm{M} \text { sucrose solution: }}$}

- 42,75 g sucrose

- Ad $50 \mathrm{ml}$ 20mM HEPES buffer

To solve the sucrose completely, the solution was carefully heated and stirred for at least $1 \mathrm{~h}$ at room temperature. 


\subsubsection{Labeling of MV through PKH26 staining}

To visualize uptake of MV into recipient cells, MV were labeled with PKH26 (Sigma). PKH26 is a red-fluorescent dye which intercalates non-specifically into lipid membranes and can be used for live cell tracking. Up to $100 \mu \mathrm{g}$ MV were incubated with $250 \mu \mathrm{l}$ working solution for 5 min under constant mixing by pipetting up and down. To stop the staining, $250 \mu \mathrm{l}$ particle-free FCS were added and incubated for $1 \mathrm{~min}$. The suspension was diluted with $500 \mu \mathrm{l}$ RPMI-1640 and MV were pelleted at $14.000 \mathrm{~g}$ and $4{ }^{\circ} \mathrm{C}$ for $35 \mathrm{~min}$. The MV pellet was washed once in PBS and then resuspended in PBS for further experiments.

\section{PKH26 working solution:}

- $250 \mu 1$ Diluent C

- $1 \mu 1 \mathrm{PKH} 26$

\subsubsection{Coupling of rhWnt5a to EV}

In order to investigate if rhWnt5a can also associate unspecifically with EV, MV or Exo $(100 \mu \mathrm{g}$ each) from the Wnt5a-negative cell line MCF-7 were incubated with $100 \mathrm{ng} / \mathrm{ml}$ rhWnt5a for $12 \mathrm{~h}$ at $37^{\circ} \mathrm{C}$. The reaction was carried out in $640 \mu 1$ particle-free MCF-7 supernatant in case that additional soluble factors in the tumor cell supernatant are required for Wnt5a binding to the vesicles. Subsequently, MV or Exo were isolated from the supernatant according to the protocol in 2.2.2.1.1, washed once in PBS and resuspended in RIPA lysis buffer for Western Blotting. As negative control, MV and Exo were incubated in particle-free MCF-7 supernatant without addition of rhWnt5a and treated as described above. To assess whether and how much of the added rhWnt5a remained unbound in the supernatant after $12 \mathrm{~h}$ of incubation, the supernatant after precipitation of MV or Exo was additionally loaded on Western Blot gels.

\subsubsection{Protein biochemistry}

\subsubsection{Protein isolation}

To isolate protein from MV and Exo preparations, pellets were resuspended in RIPA lysis buffer and subjected to protein quantification as described in 2.2.3.2.

To generate lysates from whole cells, cells were seeded in 6-well plates in a concentration of $5 \cdot 10^{5}$ cells $(\mathrm{MCF}-7, \mathrm{SK}-\mathrm{BR}-3, \mathrm{M} \phi)$ or $2 \cdot 10^{5}$ cells ((Wnt5a-) L cells) per well, allowed to 
adhere and then incubated with or without previous stimulation. After the indicated time points cells were washed once with PBS and then detached from the well with a cell scraper on ice in $100 \mu \mathrm{l}$ RIPA lysis buffer. Lysates were centrifuged for $10 \mathrm{~min}$ at $20.000 \mathrm{~g}$ and $4^{\circ} \mathrm{C}$ to pellet cell debris and DNA. Supernatants were collected and stored at $-20^{\circ} \mathrm{C}$.

\section{RIPA lysis buffer:}

- $50 \mathrm{mM}$ Tris, $\mathrm{pH} 7,2$

- $150 \mathrm{mM} \mathrm{NaCl}$

- $0,1 \%$ SDS

- $0,55 \mathrm{Na}$-deoxycholat

- $1 \%$ Triton $\mathrm{X}-100$

For protein lysates from whole cells, protease inhibitors (Sigma) as well as phosphatase inhibitors (Roche) were added to the lysis buffer.

\subsubsection{Protein quantification by Lowry assay}

The protein concentration in whole cell lysates as well as the amount of isolated MV and Exo were quantified in a Lowry assay ( $\mathrm{D}_{\mathrm{C}}$ protein assay, Bio-Rad) in relation to a BSA standard curve. Samples were diluted in $10 \mu \mathrm{l} \mathrm{ddH}_{2} \mathrm{O}$ (1:5 for MV and Exo, 1:10 for cell lysates) and the assay was carried out according to the manufacturer's instructions. The Lowry assay is based on the formation of complexes between copper (II) ions and peptide bonds of the test sample in alkaline medium. They are subsequently reduced to monovalent cations and can react with the Folin-Ciocalteu reagent which results in a blue color that can be measured in a photometer at $750 \mathrm{~nm}$ (Lowry et al, 1951; Peterson, 1979).

\subsubsection{Protein deglycosylation by PNGaseF treatment}

For the analysis of the glycosylation status of EMMPRIN, $30 \mu \mathrm{g} T-\mathrm{MV}_{\mathrm{M}}$ were incubated under non-denaturing conditions with $1000 \mathrm{U}$ Peptide N-Glycosidase F (PNGaseF, New England Biolabs) for 1 hour at $37^{\circ} \mathrm{C}$ and directly subjected to microinvasion assays or lysed in RIPA buffer for Western blotting. PNGaseF removes N-linked oligosaccharides from glycoproteins by cleaving between asparagine and $\mathrm{N}$-acetylglucosamines (Maley et al, 1989). As deglycosylation control, $\mathrm{T}_{-} \mathrm{MV}_{\mathrm{M}}$ were incubated with $20 \mathrm{U}$ PNGaseF under denaturing conditions according to the manufacturer's instructions. 


\subsubsection{SDS-PAGE}

In order to analyze the protein expression in whole cell lysates or EV by Western Blotting (see 2.2.3.5), proteins were first separated according to their molecular weight by discontinuous SDS polyacrylamide gel electrophoresis (SDS-PAGE) (Laemmli, 1970) consisting of two separate gels cast at different $\mathrm{pH}$ values. The resolving gel (10\%) was prepared and after its polymerization the stacking gel (5\%) was layered on top. Protein samples (EV: $20 \mu \mathrm{g}$, cell lysates: $50 \mu \mathrm{g}$ ) were mixed with 4x loading buffer (Roth) and heated for $5 \mathrm{~min}$ at $95^{\circ} \mathrm{C}$. On the one hand, the buffer contains $\beta$-mercaptoethanol which reduces the protein disulfide bonds and on the other hand, it comprises SDS that quantitatively binds to proteins thereby applying a negative charge in proportion to their mass. Thus, the mobility of the proteins during the electrophoresis is solely influenced by their molecular weight. Electrophoresis for Wnt5a Western Blots was carried out under non-denaturing conditions with $\beta$-mercaptoethanol-free loading buffer (bioPLUS). The prepared samples were loaded onto the gel and focused in the stacking gel for $30 \mathrm{~min}$ at $90 \mathrm{~V}$. Subsequently, proteins were resolved for around $90 \mathrm{~min}$ at $140 \mathrm{~V}$. For determination of the protein size a protein standard (Bio-Rad) was applied.

Stacking gel $(5 \%)$ :

- $1,5 \mathrm{M}$ Tris $+2 \%$ SDS (pH 6,8) $\quad 630 \mu 1$

- Acrylamide/Bisacrylamide (30\%) $830 \mu 1$

- $\operatorname{APS}(10 \% \mathrm{w} / \mathrm{v}) \quad 50 \mu \mathrm{l}$

- TEMED $5 \mu 1$

- $\mathrm{H}_{2} \mathrm{O}$ bidest. $3,45 \mathrm{ml}$

Resolving gel (10\%):

- $1,5 \mathrm{M}$ Tris $+2 \%$ SDS $(\mathrm{pH} 8,8) \quad 5 \mathrm{ml}$

- Acrylamide/Bisacrylamide (30\%) $\quad 6,7 \mathrm{ml}$

- $\operatorname{APS}(10 \% \mathrm{w} / \mathrm{v}) \quad 200 \mu \mathrm{l}$

- TEMED $20 \mu \mathrm{l}$

- $\mathrm{H}_{2} \mathrm{O}$ bidest. $\quad 7,9 \mathrm{ml}$ 
Electrophoresis buffer $(1 \mathrm{x})$ :

- $25 \mathrm{mM}$ Tris

- 192 mM Glycin

- $0,1 \%(\mathrm{w} / \mathrm{v}) \mathrm{SDS}$

- $\mathrm{H}_{2} \mathrm{O}$ bidest.
$3 \mathrm{~g}$

$14,4 \mathrm{~g}$

$1 \mathrm{~g}$

Ad 11

\subsubsection{Western Blot}

After electrophoresis, proteins were blotted onto a nitrocellulose membrane (GE Healthcare) where they then can be detected by specific antibodies. The resolving gel was put on top of the nitrocellulose membrane (Hybond-C Extra, GE Healthcare) which had been equilibrated in transfer buffer. The blot sandwich was covered by three sheets of Whatman Paper (GB33, Heinemann Labortechnik) on each side. Blotting was performed using a semi-dry transfer system at $10 \mathrm{~V}$ for $75 \mathrm{~min}$. Afterwards the membrane was routinely stained with $0,5 \%$ Ponceau S (Merck) as loading control and to confirm transfer efficiency. For protein detection, the membrane was blocked for $2 \mathrm{~h}$ in $5 \%$ milk powder (Bio-Rad) in TBST at room temperature to saturate unspecific binding sites and subsequently incubated with the specific primary antibodies (see 2.1.4) in 5\% milk powder in TBST overnight at $4^{\circ} \mathrm{C}$. After three wash steps with TBST for $5 \mathrm{~min}$ each, the membrane was incubated with secondary antibodies conjugated to horseradish peroxidase (HRP) (see 2.1.4) in 5\% milk powder in TBST for $1 \mathrm{~h}$ at room temperature and rinsed again three times in TBST for 5 min each.

\section{TBST (1x):}

- $20 \mathrm{mM}$ Tris

- $137 \mathrm{mM} \mathrm{NaCl}$

- $\mathrm{H}_{2} \mathrm{O}$ bidest.

- $0,1 \%$ (v/v) Tween-20
$2,4 \mathrm{~g}$

$8 \mathrm{~g}$

ad 11 and adjust to $\mathrm{pH} 7,6$

$1 \mathrm{ml}$

\section{$\underline{\text { Transfer buffer }(1 \mathrm{x}) \text { : }}$}

- $25 \mathrm{mM}$ Tris

- 192 mM glycine

- $20 \%(\mathrm{v} / \mathrm{v})$ methanol

- $\mathrm{H}_{2} \mathrm{O}$ bidest.
$3 \mathrm{~g}$

$14,4 \mathrm{~g}$

$200 \mathrm{ml}$

ad 11 and adjust to $\mathrm{pH} 8,3$ 
Signals were detected using the ECLPrime detection reagent (GE Healthcare) in the LAS4000 imager (Fujifilm). HRP is an enzyme that catalyzes the oxidation of luminol, thereby producing a chemoluminescence signal which can be detected in a biomolecular imager.

\subsubsection{2D-DIGE}

Using a two-dimensional difference gel electrophoresis (2D-DIGE) approach (Unlu et al, 1997) up to three different protein samples can be compared in one gel. Each sample is labeled with a different fluorescent dye so that after electrophoresis the gel can be scanned three times with the excitation wavelength of the respective dye and gel scans obtained separately for each sample. Overlay of the three images then allows identification and comparative analysis of up- or downregulated proteins. For identification of the proteins, the protein spots of interest are excised from the gel, digested with trypsin and subjected to mass spectrometry.

$\mathrm{T}_{-} \mathrm{MV}_{\mathrm{M}}$ before and after $\mathrm{N}$-deglycosylation with PNGaseF using non-denaturing conditions were labeled with the Refraction-2D ${ }^{\mathrm{TM}}$ Labeling kit (DyeAGNOSTICS). The kit contains the fluorescent G-dye 200 and G-dye 300 which covalently bind to lysine residues in protein samples. $50 \mu \mathrm{g}$ of each sample were transferred to a new reaction tube and 2D-DIGE lysis buffer was added to a final volume of $10 \mu$ l. The samples were vortexed, spun down and $1 \mu 1$ of the respective G-dye working solution ( $1 \mathrm{nmol}$ G-Dye in 2,5 $\mu \mathrm{l}$ G-Dye solvent, $\mathrm{c}=$ $400 \mathrm{pmol} / \mu \mathrm{l}$ ) was added. After incubation for $30 \mathrm{~min}$ on ice, the reaction was stopped by addition of $1 \mu \mathrm{l} \mathrm{G}$-dye labeling stop solution and incubating samples for $10 \mathrm{~min}$ on ice. To avoid dye bias, technical replicates with a dye swap were included.

\section{D-DIGE lysis buffer:}

- $30 \mathrm{mM}$ Tris

- 7 M Urea

- $2 \mathrm{M}$ Thiourea

- $4 \%(\mathrm{w} / \mathrm{v})$ CHAPS

- $\mathrm{H}_{2} \mathrm{O}$ bidest.
$0,18 \mathrm{~g}$

$21 \mathrm{~g}$

$7,6 \mathrm{~g}$

$2 \mathrm{~g}$

Ad $50 \mathrm{ml}$ and adjust to $\mathrm{pH} 8,5$

Subsequently, labeled MV samples were mixed and subjected to 2D electrophoresis which was performed by Kathrin Darm (University Medicine Greifswald). Proteins were first separated according to their isoelectric point by isoelectric focusing. Therefore, samples are loaded on an IPG strip which consists of an immobilized pH gradient. An electric potential is 
applied to the strip and since the charge of a protein is determined by the $\mathrm{pH}$ of the local surroundings, proteins will migrate along the gradient until they arrive at the $\mathrm{pH}$ value which corresponds to their isoelectric point. After the first dimension, the strips were applied to a conventional SDS-PAGE (see 2.2.3.4) which further separates the proteins according to their molecular weight. Gels were scanned with the Typhoon 9400TM laser scanner (GE Healthcare) at $554 \mathrm{~nm}$ (G-Dye 200) or $648 \mathrm{~nm}$ (G-Dye 300) and an overlay was created using the Delta2D software (DECODON GmbH). Spots were numbered and subjected to mass spectrometry as described in 2.2.3.7. Mass spectrometry data were analyzed by Dr. Christian Scharf (University Medicine Greifswald).

\subsubsection{Analysis of the protein glycosylation status by $1 \mathrm{D}$ electrophoresis and mass spectrometry}

Differences in the glycosylation pattern of the EMMPRIN glyoforms were investigated by mass spectrometry in cooperation with Kathrin Darm and Dr. Christian Scharf (University Medical Center Greifswald). T-MV $\mathrm{M}_{\mathrm{M}}$ before and after deglycosylation with PNGaseF under non-denaturing conditions (described in 2.2.3.3) were subjected to 1D SDS-PAGE. After electrophoresis all bands corresponding to the different EMMPRIN glycoforms (HG-, IG-, LG-EMMPRIN and core protein) were manually excised from the gel and sent to Greifswald for LC-MS/MS analysis. Gel bands were digested with trypsin (ratio 1:25, Promega) overnight at $37^{\circ} \mathrm{C}$ which was stopped by addition of $1 \%$ acetic acid. This digestion step is essential for the degradation of proteins which would otherwise be too large for analysis. Trypsin, a serine-protease, is very stable and has a well-defined specificity cleaving proteins at the carboxyl side of lysine and arginine residues.

Desalting of the peptides was performed with C-18 ZipTip (Millipore) according to the manufacturer's instructions. Tryptic peptides were separated by reverse phase liquid chromatography on a Proxeon Easy nano-LC system. Based on the polarity of the peptides, they bind to a hydrophobic matrix in an aqueous, polar buffer $(0.1 \%$ acetic acid, $2 \%$ acetonitrile in water) and are then eluted by using a linear gradient of a non-polar, organic solvent $(0,1 \%$ acetic acid in $100 \%$ acetonitrile). Mass spectrometry of the eluted peptides was performed on an Orbitrap Velos mass spectrometer (PicoTip Emitter, New Objective) and analyzed by Dr. Christian Scharf as described in detail in (Menck et al, 2014b). 


\subsubsection{Detection of metalloproteinase activity by zymography}

Activity of the two gelatinases MMP-2 and -9 can be determined in cell culture supernatants or cell lysates by gelatin zymography. Cell lysates $(35 \mu \mathrm{g})$, supernatants (12 $\mu \mathrm{l})$ or MV $(35 \mu \mathrm{g})$ were mixed with equal amounts of non-reducing $2 \mathrm{x}$ loading buffer without $B$ mercaptoethanol (Invitrogen) and loaded onto SDS-PAGE gels (8\%) which had been supplemented with $1 \mathrm{mg} / \mathrm{ml}$ gelatin. Supernatants (5 $\mu \mathrm{l}$ each) from MCF-7 cells stably transfected to overexpress either MMP-2 or -9 were mixed and used as standards. Electrophoresis was performed as described in 2.2.3.4 to separate proteins according to their molecular weight. Subsequently, gels were washed for $30 \mathrm{~min}$ in 2,5\% (v/v) Triton-X 100 to remove residual SDS from the electrophoresis and incubated for $30 \mathrm{~min}$ in renaturation buffer at room temperature. The buffer was exchanged and gels incubated overnight at $37^{\circ} \mathrm{C}$ to restore MMP activity. During this time, the gel is locally degraded due to the gelatinase activity of MMP-2 and -9. This degradation can be visualized by staining of the gels with Coomassie Blue. For the staining, gels were incubated for $4 \mathrm{~h}$ in stain/destain solution (1:10). To reduce background staining, gels were washed in destain solution until the gelatinase bands were clearly visible and fixed for $15 \mathrm{~min}$ in fixative solution before gels were dried.

\section{$\underline{\text { Renaturation buffer: }}$}

- $50 \mathrm{mM}$ Tris

- $200 \mathrm{mM} \mathrm{NaCl}$

- $5 \mathrm{mM} \mathrm{CaCl}_{2}$

- $\mathrm{H}_{2} \mathrm{O}$ bidest.

- $0,02 \%(\mathrm{v} / \mathrm{v})$ Brij-35
$6,06 \mathrm{~g}$

$11,7 \mathrm{~g}$

$0,56 \mathrm{~g}$

ad 11 and adjust to $\mathrm{pH} 7,5$

$0,67 \mathrm{ml}$

Stain solution:

- $0,5 \%(\mathrm{w} / \mathrm{v})$ Coomassie Brilliant Blue $5 \mathrm{~g}$

- $30 \%(\mathrm{v} / \mathrm{v})$ methanol $300 \mathrm{ml}$

- $10 \%(\mathrm{v} / \mathrm{v})$ acetic acid $\quad 100 \mathrm{ml}$

- $\mathrm{H}_{2} \mathrm{O}$ bidest. $\quad \mathrm{ad} 11$

Destain solution:

- $30 \%(\mathrm{v} / \mathrm{v})$ methanol $\quad 300 \mathrm{ml}$

- $10 \%(\mathrm{v} / \mathrm{v})$ acetic acid $\quad 100 \mathrm{ml}$

- $\mathrm{H}_{2} \mathrm{O}$ bidest. $\quad$ ad 11 
Fixative solution:

- $30 \%(\mathrm{v} / \mathrm{v})$ methanol

$300 \mathrm{ml}$

- $5 \%(\mathrm{v} / \mathrm{v})$ glycerol

$50 \mathrm{ml}$

- $\mathrm{H}_{2} \mathrm{O}$ bidest.

ad 11

\subsubsection{Immunological methods}

\subsubsection{Flow cytometry}

\subsection{Characterization of MV by flow cytometry}

For characterization of MV by flow cytometry, MV (6 $\mu \mathrm{g})$ were transferred to round-bottom FACS tubes (BD) and incubated in $14 \mu \mathrm{l}$ PBS $+1 \%$ particle-free FCS for 15-30 min at room temperature to saturate unspecific binding sites. The fluorochrom-labeled antibodies were added at the indicated concentrations (see Tab. 4) and samples stained for $20 \mathrm{~min}$ at room temperature in the dark. For unlabeled primary antibodies the staining was performed at $26^{\circ} \mathrm{C}$ for $40 \mathrm{~min}$, MV washed twice in PBS and signals detected by incubation with FITC-labeled anti-mouse or anti-rabbit secondary antibodies (\#406403, BioLegend; \#sc-2010, santa cruz) for $20 \mathrm{~min}$ at room temperature in the dark. Irrelevant IgG1 or IgG2b antibodies were used as respective isotype-matched negative controls. MV were resuspended in $250 \mu \mathrm{PBS}$ and fluorescence was measured with a FACSCanto II flow cytometer (BD Biosciences) using the FACSDiva software version 6.1.3 (BD Biosciences). Forward scatter (FSC) versus side scatter (SSC) dot plots were used to gate the MV population which was then characterized further. Histogram overlays were created with GIMP for Windows version 2.8.

\subsection{Characterization of Exo by flow cytometry}

Since Exo are too small to be detected by a normal flow cytometer, they have to be coupled to larger latex beads $(\varnothing 4 \mu \mathrm{m})$ prior to analysis. For the preparation of the latex beads stock solution $30 \cdot 10^{6}$ aldehyde/sulfate beads $(23,08 \mu 1$, Invitrogen) are transferred to a reaction tube and centrifuged for $20 \mathrm{~min}$ at $3000 \mathrm{~g}$. The pellet was washed twice in MES buffer and resuspended in $100 \mu \mathrm{MES}$ buffer (c=300.000 Beads/ $\mu 1)$.

$10 \mu \mathrm{g}$ Exo per sample were incubated with $1 \mu \mathrm{l}$ of the beads stock solution in $300 \mu \mathrm{MES}$ buffer for $1 \mathrm{~h}$ at room temperature with gentle shaking. After addition of $300 \mu 1$ MES buffer, samples were put in a tube rotator and gently agitated over night at $4^{\circ} \mathrm{C}$. To stop the coupling reaction, $400 \mu \mathrm{l}$ glycine $(200 \mathrm{mM})$ were added followed by incubation at room temperature 
for 30 min. Exo-Bead complexes were washed twice in PBS +0,5\% particle-free FCS for $10 \mathrm{~min}$ at $2.500 \mathrm{~g}$ and then subjected to staining and subsequent flow cytometric analyses as described in 2.2.4.1.1.

MES buffer:

- 0,025 M MES

- $0,154 \mathrm{M} \mathrm{NaCl}, \mathrm{pH} 6,0$

\subsection{Characterization of cells by flow cytometry}

For immunophenotyping of human $\mathrm{M} \phi$, cells were seeded in 6-well plates at a concentration of $1 \cdot 10^{6}$ cells per well and allowed to adhere overnight. They were washed twice with PBS, carefully scraped from the surface of the well and transferred to FACS tubes. Cells were pelleted at $750 \mathrm{~g}$ and incubated in PBS $+1 \%$ particle-free FCS for $15 \mathrm{~min}$ at room temperature. The staining procedure and flow cytometry were performed as described in 2.2.4.1.1.

\subsection{Determination of MV counts by flow cytometry}

For the determination of MV counts in samples which had been resuspended in equal volumes of PBS, $6 \mu 1$ of each sample were transferred to FACS tubes. After addition of $250 \mu 1$ PBS and $10 \mu \mathrm{l}$ Flow-Set Beads (Ø 3,3 $\mu \mathrm{m}$, Beckman Coulter) the samples were measured in a FACSCanto II flow cytometer. Next to the routinely used MV gate, a second gate was defined around the population of Flow-Set Beads. In each sample 1000 events in the Beads gate were recorded and the numbers of MV which had been counted meanwhile were compared. Each measurement was performed in triplicate.

\subsection{Measurement of MV uptake by flow cytometry}

For uptake studies, cells were seeded in 6-well plates (human M $\phi: 1 \cdot 10^{6}$, MCF-7: $5 \cdot 10^{5}$ ) and pre-incubated for $2 \mathrm{~h}$ with $1 \mu \mathrm{M} Z \mathrm{ZA}, 1,25 \mu \mathrm{M}$ filipin III, $25 \mu \mathrm{M}$ dansylcadaverine or the indicated concentrations of dynasore before $10 \mu \mathrm{g}$ PKH26-labeled T-MV $\mathrm{M}_{\mathrm{M}}$ were added. After $24 \mathrm{~h}$ of stimulation, cells were washed once in PBS and carefully scraped from the wells in $500 \mu \mathrm{l} \mathrm{PBS}$. The red fluorescence of the population of intact cells was measured in a FACSCanto II flow cytometer. 


\subsubsection{Calcein staining of MV and cells}

The calcein staining is based on the metabolization of calcein-AM to green fluorescent calcein. This reaction is catalyzed by esterases in the cytoplasm and only takes place in viable cells with an intact plasma membrane. Human M $\phi\left(1 \cdot 10^{6}\right)$ or MV $(25 \mu \mathrm{g})$ were incubated in $1000 \mu \mathrm{l}$ PBS $+2 \mu \mathrm{l}$ calcein-AM (Fluo Cell double staining kit, MoBiTec) for $15 \mathrm{~min}$ at $37^{\circ} \mathrm{C}$. Subsequently, they were washed once with PBS and directly subjected to confocal microscopy (2.2.6.2) or measured by flow cytometry (2.2.4.1.1). As negative control for flow cytometry assays, MV were incubated for $3 \mathrm{~h}$ in $2 \mathrm{ml}$ acetone to destroy membrane integrity before calcein staining was performed.

\subsubsection{Separation of T-EV by MACS}

For separation of T-EV from human serum samples, the latter were prepared as described in 2.1.1.3 or 2.2.2.1.3, respectively, and the supernatant after the centrifugation step at $1500 \mathrm{~g}$ stored for up to 2 weeks at $-20^{\circ} \mathrm{C}$. To $5 \mathrm{ml}$ of each serum sample 5,10 or $50 \mu \mathrm{l}$ magnetically labeled anti-EpCAM MicroBeads (Miltenyi Biotec) were added and samples incubated for $2 \mathrm{~h}$ at $4^{\circ} \mathrm{C}$ under gentle movement for antibody binding. Subsequently, they were applied to LD or LS columns onto a MACS Vario separator (Miltenyi Biotec) which had been equilibrated with $2 \times 1 \mathrm{ml}$ MACS buffer. Due to the strong magnetic field in the separator, the labeled EV are captured in the column, while all EpCAM-negative EV are eluted. The flow-through was collected for EV isolation and used as negative control. The EpCAM-positive sorted EV bound to the column were eluted in $5 \mathrm{ml}$ MACS buffer after removal of the column from the separator and also employed for EV isolation as described in 2.2.2.1.1.

\section{MACS buffer:}

- 2 mM EDTA

- $0,5 \%$ particle-free FCS

- Ad $100 \mathrm{ml}$ PBS, adjust to $\mathrm{pH} 7,2$

\subsubsection{Measurement of cytokine concentrations by ELISA}

Concentrations of IL-1ß, IL-10 and IL-12 (p70) in supernatants of human M $\phi$ were measured with Legend $\operatorname{Max}^{\mathrm{TM}}$ ELISA kits (\#437007, \#430607, \#431707, all three from BioLegend) with pre-coated plates. Briefly, human $\mathrm{M} \phi$ were seeded in 6-well plates at a concentration of $5 \cdot 10^{5}$ cells per well, washed twice with PBS and stimulated for $24 \mathrm{~h}$ with LPS (100 ng/ml), $\mathrm{T}_{-} \mathrm{MV}_{\mathrm{M}}$ or P-MV (both $25 \mu \mathrm{g} / \mathrm{ml}$ ) in $2 \mathrm{ml} \mathrm{RPMI}+1 \%$ particle-free FCS. Supernatants were 
collected and centrifuged at $2.000 \mathrm{~g}$ for $5 \mathrm{~min}$ to remove residual cells and debris. The ELISA tests were carried out according to the manufacturer's instructions. Samples $(100 \mu l)$ were measured in triplicate and concentrations calculated in relation to an IL-1B, IL-10 or IL-12 standard curve.

\subsubsection{Gene expression analysis}

\subsubsection{Isolation of total RNA from human tissue samples}

The isolation of total RNA from human brain metastases samples was carried out with the TRIzol (Invitrogen) reagent. First, small tissue samples were homogenized in $1 \mathrm{ml}$ TRIzol, before $200 \mu \mathrm{l}$ chloroform were added, mixed and incubated for $5 \mathrm{~min}$ at room temperature. Centrifugation at $20.000 \mathrm{~g}$ for $15 \mathrm{~min}$ at $4^{\circ} \mathrm{C}$ yields three phases with the RNA being present in the colorless upper aqueous phase. The upper phase was transferred to a new tube and $500 \mu \mathrm{l}$ isopropanol were added. For the precipitation of the RNA, the sample was incubated for $10 \mathrm{~min}$ at room temperature followed by centrifugation at $13.000 \mathrm{~g}$ and $4^{\circ} \mathrm{C}$ for $30 \mathrm{~min}$. The pellet was washed in $1 \mathrm{ml} 70 \%$ ethanol at $20.000 \mathrm{~g}$ and $4^{\circ} \mathrm{C}$ for $5 \mathrm{~min}$ and afterwards air dried for 5-10 min. To remove contaminating DNA, the pellet was resuspended in $50 \mu 1$ DNA digestion mix and incubated for $20 \mathrm{~min}$ at $37^{\circ} \mathrm{C}$. To purify the remaining RNA, $150 \mu \mathrm{l}$ nuclease-free water and $200 \mu \mathrm{l}$ phenol/chloroform/isoamyl alcohol were added. The sample was vortexed for $30 \mathrm{sec}$ and subsequently centrifuged for $2 \mathrm{~min}$ at $20.000 \mathrm{~g}$ and $4^{\circ} \mathrm{C}$. The upper aqueous phase which contains the RNA was transferred to a new tube. $20 \mu 1$ sodium acetate $(3 \mathrm{M}, \mathrm{pH} 4,8)$ and $200 \mu \mathrm{l}$ isopropanol were added and the sample centrifuged for $30 \mathrm{~min}$ at $20.000 \mathrm{~g}$ and $4^{\circ} \mathrm{C}$ to precipitate the RNA. The pellet was washed twice in $1 \mathrm{ml} 70 \%$ ethanol for $5 \mathrm{~min}$ each at $20.000 \mathrm{~g}$ and $4^{\circ} \mathrm{C}$ and was air dried afterwards. The RNA was resuspended in $15 \mu 1$ nuclease-free water and the concentration and purity determined at the NanoDrop ND-1000 spectrophotometer (Peqlab).

\section{DNA digestion mix:}

- $1 \mu 1$ DNase I (10 U/ $\mu 1$, Roche)

- $0,5 \mu 1$ RNase OUT (40 U/ $\mu 1$, Invitrogen)

- $5 \mu 1$ DNase I incubation buffer (10x, Roche)

- Ad 50 $\mu 1$ nuclease-free water 


\subsubsection{Isolation of mRNA from eukaryotic cells and vesicles}

The isolation of mRNA from cultured cells and MV was carried out with the spin columnbased High Pure RNA isolation kit (Roche) according to the manufacturer's instructions. Briefly, the cells were seeded in a concentration of $5 \cdot 10^{5}$ cells per well in 6-well plates and stimulated as indicated. Human $\mathrm{M} \phi$ were seeded at a concentration of $1 \cdot 10^{6}$ cells on wells previously coated with $80 \mu \mathrm{I}$ ECM (diluted 1:16 in serum-free RPMI-1640). Subsequently, cells were washed once with PBS and as well as MV pellets lysed in $400 \mu \mathrm{l}$ of the lysis/binding buffer supplemented with $200 \mu \mathrm{l}$ PBS. While RNases are inactivated, the buffer further contains Triton X-100 which mediates permeabilization of the cell membrane and guanidine hydrochloride which induces protein denaturation. The samples were vortexed for $15 \mathrm{sec}$ to help cell lysis and subsequently applied onto a spin column that consists of glass fiber fleece. Columns were centrifuged at $8000 \mathrm{~g}$ for $15 \mathrm{sec}$ which leads to the binding of the nucleic acids to the column, while proteins, salts and cellular debris are eluted. Contaminating DNA was digested directly on the column by incubation with DNase I for $15 \mathrm{~min}$. The remaining RNA was washed three times, before $50 \mu 1$ nuclease-free water were added to the column for elution of the isolated RNA. Concentration and purity were measured with the NanoDrop ND-1000.

\subsubsection{Reverse transcription}

For the analysis of gene expression changes by qRT-PCR, the isolated RNA was transcribed into complementary DNA (cDNA) using the iScript cDNA synthesis kit (Bio-Rad). The reaction is carried out by the reverse transcriptase, an RNA-dependent DNA polymerase. The enzyme binds to the random hexamer oligonucleotides and oligo(dT) primers which are contained in the kit. The latter are complementary to the poly-A tail of eukaryotic mRNAs allowing reverse transcription of all mRNAs in the sample. The reaction setup was prepared as follows:

$\begin{array}{lc}\text { 5x iScript reaction mix } & 4 \mu \mathrm{l} \\ \text { iScript reverse transcriptase } & 1 \mu \mathrm{l} \\ \text { RNA template }(1 \mu \mathrm{g}) & \mathrm{x} \mu \mathrm{l} \\ \text { Nuclease-free water } & \mathrm{y} \mu \mathrm{l}\end{array}$


The prepared reaction mix was incubated in a thermal cycler (Biometra) for $5 \mathrm{~min}$ at $25^{\circ} \mathrm{C}$ followed by $30 \mathrm{~min}$ at $42^{\circ} \mathrm{C}$ and $5 \mathrm{~min}$ at $85^{\circ} \mathrm{C}$. Each sample was diluted in a ratio of $1: 5$ with nuclease-free water and stored at $-20^{\circ} \mathrm{C}$.

\subsubsection{Quantitative real-time PCR (qRT-PCR)}

Changes in gene expression were analyzed by quantitative real-time PCR (qRT-PCR) using SYBR green detection. The basic principle of this method is a conventional PCR reaction. After activation of the hot-start polymerase, the gene of interest is amplified during 40 cycles of DNA denaturation, followed by annealing of gene-specific primers and extension of the product. Every cycle is completed by measurement of the fluorescence of SYBR green which is an intercalating dye that emits a fluorescent signal when bound to double-stranded DNA. Since the copy number of the gene of interest is amplified with every cycle, the fluorescence increases proportionally and can be plotted versus the cycle number. The number of the cycle in which the fluorescence reaches the threshold level is called $C_{t}$ value and was normalized on the expression of two house-keeping genes $\left(=\Delta \mathrm{C}_{\mathrm{t}}\right.$ value). For relative quantification, the $\Delta \mathrm{C}_{\mathrm{t}}$ values were related to an unstimulated control $\left(=\Delta \Delta \mathrm{C}_{\mathrm{t}}\right.$ value $)$ which can be used to calculate the fold change of the gene of interest according to following equation:

$$
\text { fold change }=2^{-\Delta \Delta \mathrm{Ct}}
$$

The primers used in this study were designed as described in 2.2.5.4.1 and are listed in Tab. 5. Human samples were normalized on HPRT1 and GNB2L1 expression, murine samples on GAPDH and TBP expression. For each gene $8 \mu \mathrm{l}$ from the prepared PCR reaction mix were pipetted into a 384-well plate (Greiner Bio-One) in triplicates. To each well $2 \mu \mathrm{l}$ cDNA (10ng) diluted in nuclease-free water were added. The plate was sealed, spun down (2 min, $750 \mathrm{~g}$ ) and RT-PCR was carried out according to the following protocol in the Taqman ABI PRISM 7900 HT detection system (Applied Biosystems).

\section{Standard qRT-PCR program:}

- Activation of taq-polymerase:

\begin{tabular}{ll|l}
$95^{\circ} \mathrm{C}$ & $12 \mathrm{~min}$ & \\
$95^{\circ} \mathrm{C}$ & $15 \mathrm{sec}$ & 40 cycles \\
$60^{\circ} \mathrm{C}$ & $1 \mathrm{~min}$ & \\
$95^{\circ} \mathrm{C}$ & $15 \mathrm{sec}$ \\
$60-95^{\circ} \mathrm{C}$ & $2^{\circ} \mathrm{C} / \mathrm{min}$
\end{tabular}

- Denaturation:

- Annealing and elongation: 
$\underline{10 \times \text { PCR buffer: }}$

- $\quad$ Tris- $\mathrm{HCl} \mathrm{pH} 8,8 \quad 0,75 \mathrm{M}$

- Ammoniumsulfate $0,2 \mathrm{M}$

- Tween-20 0,1\% (v/v)

\section{$\underline{\text { SYBR-Green master mix: }}$}

All solutions were prepared in nuclease-free water, except the trehalose solution which was resuspended in $10 \mathrm{mM}$ Tris- $\mathrm{HCl} \mathrm{pH} 8,0$.

- 10x PCR buffer

- $25 \mathrm{mM} \mathrm{MgCl} 2$

- SYBR green $(1: 100)$

- $20 \mathrm{mM}$ dNTP mix

- $5 \mathrm{U} / \mu 1 \mathrm{Taq}$ polymerase

- $10 \%$ Triton $\mathrm{X}-100$

- $1 \mathrm{M}$ trehalose

PCR reaction mix:

- SYBR green master mix $5,6 \mu 1$

- fw-primer $(10 \mu \mathrm{M})$ $0,3 \mu 1$

- rv-primer $(10 \mu \mathrm{M})$ $0,3 \mu 1$

- nuclease-free water

$$
\begin{aligned}
& 2,5 \mathrm{ml} \\
& 3 \mathrm{ml} \\
& 31.3 \mu \mathrm{l} \\
& 250 \mu \mathrm{l} \\
& 100 \mu \mathrm{l} \\
& 652 \mu \mathrm{l} \\
& 7,5 \mathrm{ml}
\end{aligned}
$$

The qRT-PCR was analyzed with SDS version 2.4 (Applied Biosystems) and fold changes or $\Delta \mathrm{C}_{\mathrm{t}}$ values plotted with GraphPad Prism for Windows (version 5.04, GraphPad software).

\subsection{Establishment of primers for $q R T-P C R$ reactions}

Primer pairs for qRT-PCR analyses were designed with PerlPrimer version 1.1.21 (Marshall, 2004) and then tested for their efficiency. Since analysis of relative gene expression by the $\Delta \Delta \mathrm{C}_{\mathrm{t}}$-method assumes an amplification efficiency of $100 \%$, comparable primer efficiencies are important. A primer efficiency of $100 \%$ means that during each PCR cycle the PCR product is exactly doubled. To test primer efficiency, a cell line or tissue with high expression of the gene of interest was chosen from the BioGPS database (Wu et al, 2009) and a serial dilution series of the cDNA with known concentrations was prepared. The measured $\mathrm{C}_{\mathrm{t}^{-}}$ values were plotted versus the amount of input cDNA and the slope of the resulting graph 
calculated with SDS version 2.4. A slope of $-3,33$ equals a primer efficiency of $100 \%$. Primers with an efficiency of 90-110\% were chosen for further analysis. Moreover, for each primer pair the melting curve was analyzed and the PCR product visualized on a $2 \%$ agarose gel to verify that a single gene product with the correct size was generated.

\subsection{Visualization of $q R T-P C R$ products on agarose gels}

Due to their negative charge DNA fragments can easily be separated according to their size by agarose gel electrophoresis. This was used to visualize gene-specific products which have been amplified during qRT-PCR. Briefly, 2\% agarose (HEEO Ultra, Roth) were added to $100 \mathrm{ml}$ TAE buffer (1x) and boiled up in a microwave until the agarose was dissolved. The solution was allowed to cool down for 3-5 min and 0,1\% (v/v) SERVA DNA Stain G (Serva) were added. This dye intercalates into nucleic acids which leads to the emission of green fluorescence. The agarose solution was poured into a horizontal gel tray and incubated until polymerization was finished after $30 \mathrm{~min}$.

The triplicates of each gene or sample of interest were pooled after successful qRT-PCR reaction and transferred to new reaction tubes. $10 \mu 1$ of each sample were mixed with $2 \mu 16 x$ loading buffer (Fermentas) and loaded onto the agarose gel. To confirm the correct size of the amplified gene products, a molecular weight ladder (Fermentas) was additionally applied to the gel. Electrophoresis was performed in a horizontal electrophoresis chamber (BiotecFischer) at $100 \mathrm{~V}$ for $45 \mathrm{~min}$ and gene-specific products subsequently visualized on an UV Transilluminator (Biometra).

10x TAE buffer:

- 0,4 M Tris

- $0,2 \mathrm{M}$ acetic acid

- 0,01 M EDTA

- $\quad$ ad $11 \mathrm{H}_{2} \mathrm{O}$ bidest and adjust $\mathrm{pH}$ to 8,5

\subsubsection{Microscopy}

\subsubsection{Fluorescence microscopy}

MV uptake into MCF-7 or SK-BR-3 cells was evaluated by fluorescence microscopy. Therefore, MV were labeled with the red fluorescent dye PKH26 as described in 2.2.2.3. $5 \cdot 10^{5}$ tumor cells were seeded in 6 -well plates followed by stimulation with $10 \mu \mathrm{g}$ PKH $26^{+}$ 
MV for $24 \mathrm{~h}$. Uptake was visualized using the Axiovert 200M fluorescence microscope (Zeiss). For quantitative analyses particular care was taken to record all images with the same exposure time.

\subsubsection{Confocal microscopy}

Uptake of $\mathrm{T}-\mathrm{MV}_{\mathrm{M}}$ into $\mathrm{M} \phi$ was analyzed by confocal microscopy to ensure that the vesicles were indeed present inside the cells and did not only stick to the cellular surface. Human $\mathrm{M} \phi$ were seeded in 6-well plates $\left(5 \cdot 10^{5}\right.$ per well), washed twice with PBS and stimulated with $10 \mu \mathrm{g}$ PKH26 ${ }^{+}$T-MV for $24 \mathrm{~h}$. Afterwards, cells were washed twice with PBS and the cytoplasm of the $\mathrm{M} \phi$ was counterstained with calcein-AM (2.2.4.2). Pictures were taken at the LSM 510 Meta confocal microscope (Zeiss).

\subsubsection{Electron microscopy}

The electron microscopy of MV was performed by Dr. Dirk Wenzel (Max Planck Institute for Biophysical Chemistry, Göttingen) and Exo were analyzed by Dr. Julia Gross (German Cancer Research Center, Heidelberg). Vesicle pellets were fixed in 2\% paraformaldehyde and applied to carbon-coated grids (400 mesh, Plano $\mathrm{GmbH})$. After postfixation in $1 \%$ glutaraldehyde, the grid was washed in $\mathrm{dH}_{2} \mathrm{O}$. Negative contrasting was achieved by incubating the grid for $30 \mathrm{~s}$ in $1 \%$ uranyl acetate and EV visualized with the CM 120 Bio Twin transmission electron microscope (Philips) and iTEM software (Olympus). For immunogold staining of Wnt5a, MV pellets were stained with rat anti-Wnt5a primary antibody (\#MAB645, R\&D systems) followed by detection with an immunogold-labeled secondary antibody (\#810.077, Aurion).

\subsubsection{Statistical analysis}

All experiments were performed at least in biological triplicates. Data are displayed as means \pm SD and statistical significance was calculated with a two-sided student's t-test, unless indicated otherwise. P-values $<0,05$ were considered significant. Data were plotted with GraphPad Prism for Windows (version 5.04, GraphPad software). Significance for patient data was calculated by Dr. Annalen Bleckmann (University Medical Center Göttingen, Dept. Hematology/Oncology). 


\section{Results}

The aim of this thesis was to investigate the influence of tumor-derived extracellular vesicles (T-EV) on the local tumor microenvironment, especially regarding their impact on the invasiveness of breast cancer cells.

Therefore, the first part of the thesis focusses on the role of T-EV for the intercellular communication between neighboring tumor cells. In this context, we tried to elucidate the molecular mechanisms which are responsible for the phenotypic differences between different microvesicle (MV) populations and to identify markers which are characteristic for proinvasive MV. The major part of this subproject is to be published in (Menck et al, 2014b).

In the second part of the thesis we further investigated whether EV are also involved in the pro-invasive interactions between tumor cells and macrophages $(\mathrm{M} \phi)$. In this context, we analyzed the influence of tumor-derived EV for the induction and transport of the tumorpromoting factor Wnt5a. Special attention was paid to the protein Evi/WLS and its significance for the expression of Wnt5a on MV. Moreover, we investigated the effect of the bisphosphonate zoledronic acid (ZA) on MV-mediated tumor-M $\phi$ crosstalk. Most of the results have been published in (Menck et al, 2013) and (Rietkotter et al, 2013).

In a translational approach we then tried to establish a reproducible method to isolate EV from peripheral blood which is described in the last part of the thesis. T-EV were screened in vitro for the expression of suitable tumor markers which were then conferred to EV isolated from metastatic cancer patients and used to identify T-MV in the blood of these patients.

\subsection{EV as mediators of tumor-tumor interactions}

The fact that cells release extracellular vesicles into the extracellular space is already known for a long time. However, they were always considered as cellular debris and are only recently increasingly accepted as novel means of intercellular communication. We hypothesized that T-EV play an important role in tumor-tumor crosstalk and aimed to investigate their mode of action. Since MV are far less investigated than Exo, we further tried to define MV-associated tumor markers which allow their discrimination from Exo. 


\subsubsection{Tumor cells release two distinct populations of extracellular vesicles}

In order to investigate if our EV isolation protocol indeed allows the preparation of two distinct vesicle populations, MV and Exo were isolated from cell culture supernatants of MCF-7 (T-MV $\mathrm{M}_{\mathrm{M}}$ and T-Exo $\mathrm{M}_{\mathrm{M}}$ ) and SK-BR-3 (T-MV $\mathrm{V}_{\mathrm{S}}$ and T-Exos) breast cancer cells as well as from outdated ( $<1$ day) platelet concentrates (P-MV) and the benign mammary epithelial cell line hTERT-HME1 (hTERT-MV). Since there is increasing evidence that the vesicular cargo is modulated by the state of the cell of origin (Mause \& Weber, 2010), all vesicles were obtained under basal conditions without any previous stimulation of the cells which could alter the EV protein expression pattern. Moreover, particular care was taken to use only viable cells to prevent the release of apoptotic bodies from the tumor cells.

A

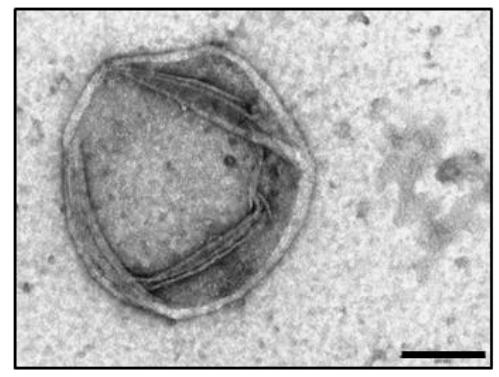

B

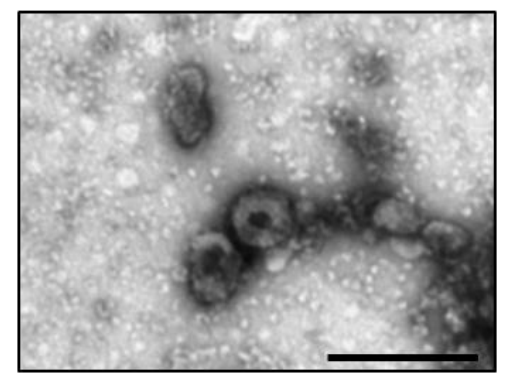

C

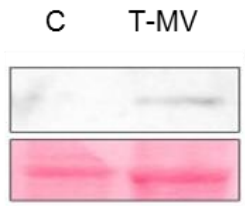

C T-Exo

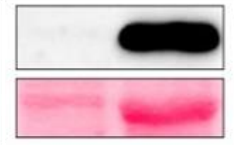

Tsg101 (46kDa)

Ponceau

D

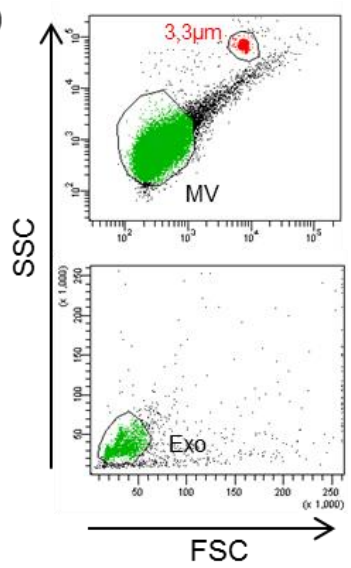

E

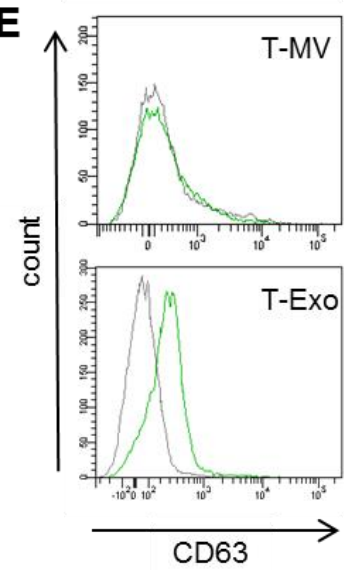

$\mathbf{F}$

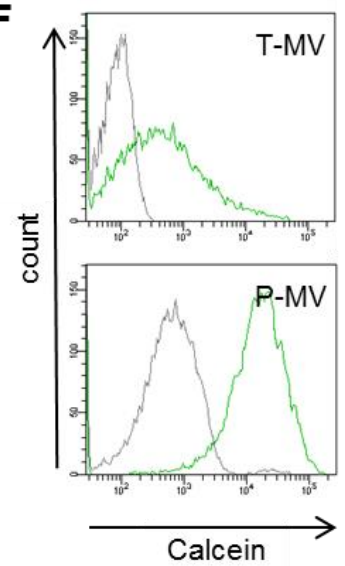

Fig. 7: MV and Exo are different populations of extracellular vesicles

$\boldsymbol{A}+\boldsymbol{B}$, TEM images of T-MV (A) and T-Exo $(B)$ preparations showing two different types of extracellular vesicles (scale bars: $200 \mathrm{~nm}$ ). $\boldsymbol{C}$, Expression of the MVB protein Tsg101 was analyzed on MV and Exo from SK-BR-3 cells in comparison to whole cell lysates (C) by Western Blotting. $\boldsymbol{D}-\boldsymbol{F}$, MV and Exo were analyzed by flow cytometry: Forward scatter (FSC) versus side scatter (SSC) plots were used to gate the respective MV or Exo population $(D)$ which was then used for further characterization. As size control for MV gating, calibration beads $(3,3 \mu \mathrm{m}$, indicated in red) were used. MV and Exo were characterized for the expression of the known Exo marker CD63 $(E)$ and membrane integrity of MV was determined by staining with calcein-AM $(F)$. Specific signals are indicated in green, corresponding isotype controls in grey. Modified from (Menck et al, 2013). 
Transmission electron microscopy (TEM) revealed two distinct populations of extracellular vesicles: 1) MV consisting of a population of very heterogeneous, large vesicles with a diameter > $100 \mathrm{~nm}$ which neither contained cell organelles nor fragmented DNA (Fig. 7A) thereby excluding the presence of apoptotic bodies; 2) typically cup-shaped Exo with a diameter of $<100 \mathrm{~nm}$ (Fig. 7B).

Moreover, the preparations were analyzed for the expression of known Exo marker proteins (Fig. 7C-E). Tsg101, typically associated with multivesicular bodies (MVB), as well as the intracellular tetraspanin CD63 were found exclusively on Exo preparations, while they were not present on MV. This confirms that MV and Exo not only differ in their morphology, but also in their protein content. To investigate if membrane integrity of the vesicles was maintained during the isolation process, we stained $\mathrm{T}-\mathrm{MV}_{\mathrm{M}}$ as well as P-MV with calcein$\mathrm{AM}$ which is converted by cytoplasmic esterases to green fluorescent calcein. Indeed, both MV populations were positive for calcein, confirming the presence of intact membrane vesicles (Fig. 7F).

\subsubsection{T-MV enhance human breast cancer invasion}

While T-Exo have already been desribed to increase cancer cell invasion (Graves et al, 2004; Higginbotham et al, 2011), little is known about the effect of T-MV on tumor progression. To address this question, we stimulated MCF-7 and SK-BR-3 breast cancer cells with autologous and heterologous T-MV $(10 \mu \mathrm{g} / \mathrm{ml})$ as well as with the corresponding vesicle-free supernatants which neither contain MV nor Exo. Stimulation with MV isolated from benign, immortalized hTERT-HME1 mammary epithelial cells (hTERT-MV) was employed as negative control. Interestingly, T-MV were able to enhance tumor invasion by up to $400 \%$, whereas neither hTERT-MV nor the vesicle-free supernatants influenced tumor invasion (Fig. $8 \mathrm{~A}+\mathrm{B}$ ) indicating that the effect is a special feature of $\mathrm{T}-\mathrm{MV}$ populations. T-Exom also significantly increased MCF-7 invasion, although the effect was clearly less pronounced compared to MV (Fig. 8A). For this reason, we decided to focus further investigations regaring tumor-tumor crosstalk on T-MV.

To exclude that the observed effect was due to an influence of T-MV on cancer cell proliferation, we seeded MCF-7 cells on ECM-coated 24-well plates and stimulated them with $\mathrm{T}-\mathrm{MV}_{\mathrm{M}}(10 \mu \mathrm{g} / \mathrm{ml})$ for $96 \mathrm{~h}$. However, the proliferation rate of the cells remained unchanged (Fig. 8C). Dilution experiments revealed that already $0,1 \mu \mathrm{g} / \mathrm{ml} \mathrm{T}-\mathrm{MV}_{\mathrm{M}}$ are sufficient to exert a pro-invasive influence on MCF-7 cells (Fig. 8D) in microinvasion assays. 
Taken together, the finding that T-MV stimulate tumor invasion in a dose-dependent manner, while hTERT-MV have no such effect, confirms that MV-induced tumor invasion is indeed a specific and not an unphysiological, artificial effect.
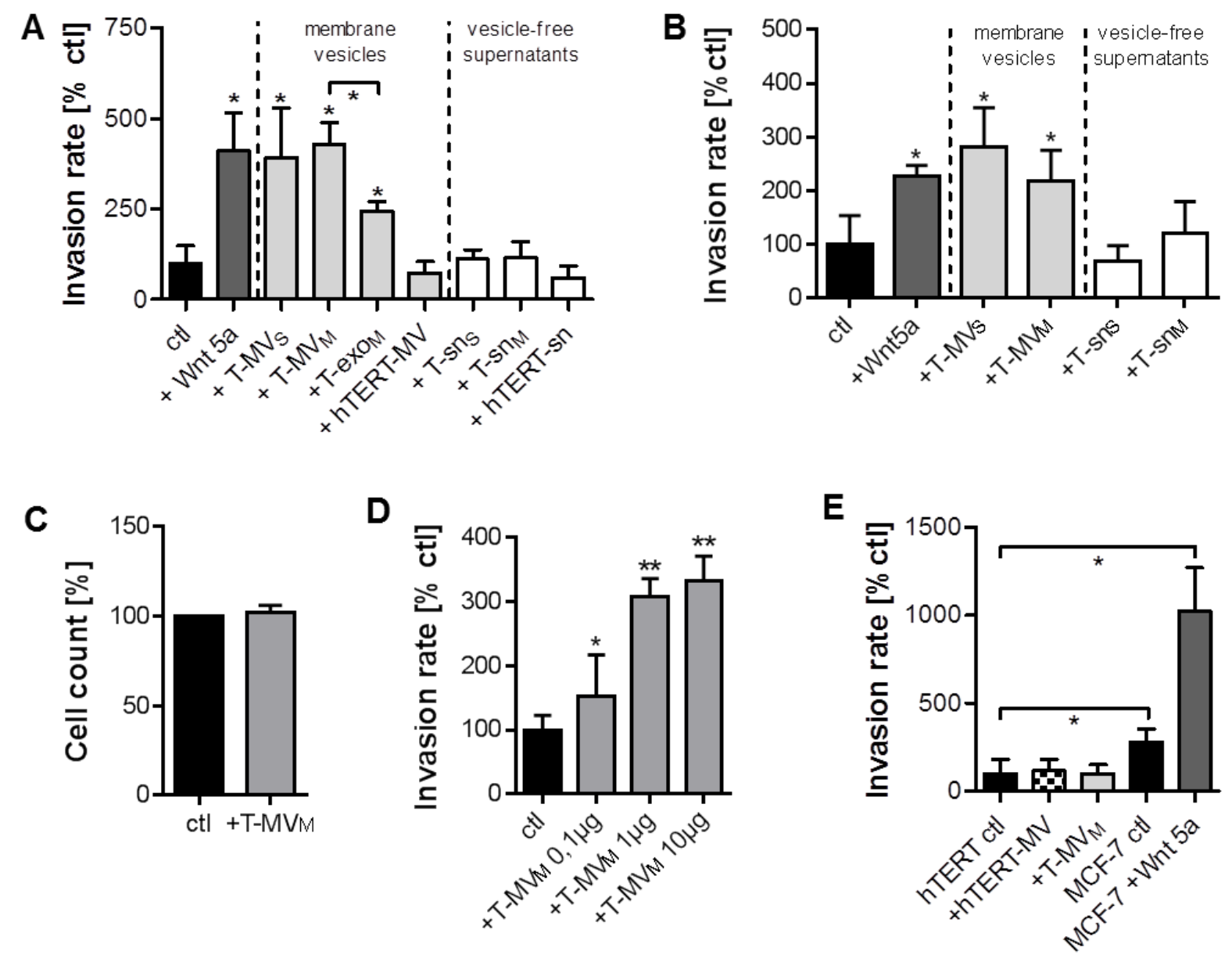

Fig. 8: T-MV enhance tumor invasion in an autologous and heterologous way

$\boldsymbol{A}+\boldsymbol{B}$, Microinvasion assays of MCF-7 (A) and SK-BR-3 (B) cells stimulated with T-MV (10 $\mu \mathrm{g} / \mathrm{ml})$, T-Exo $(10 \mu \mathrm{g} / \mathrm{ml})$ or the corresponding vesicle-free supernatants (T-sn, diluted 1:3 in culture medium). Benign hTERTMV $(10 \mu \mathrm{g} / \mathrm{ml})$ were used as negative control, stimulation with $\mathrm{rhWnt5a}(100 \mathrm{ng} / \mathrm{ml})$ was employed as positive control (means $\left.\pm \mathrm{SD}, \mathrm{n}=3,{ }^{*} \mathrm{p}<0,001\right)$. $\boldsymbol{C}$, T-MV have no influence on MCF-7 proliferation as determined by cell counting experiments (means $\pm \mathrm{SD}, \mathrm{n}=3$ ). $\boldsymbol{D}$, T-MV were able to enhance MCF-7 cell invasion in a dosedependent manner (microinvasion assays, means $\pm \mathrm{SD}, \mathrm{n}=3, * \mathrm{p}<0,05, * * \mathrm{p}<0,001$ ). $\boldsymbol{E}$, Microinvasion assay of hTERT-HME1 cells stimulated with autologous hTERT-MV or heterologous T-MV (both $10 \mu \mathrm{g} / \mathrm{ml}$ ). MCF-7 cells \pm rhWnt5a $(100 \mathrm{ng} / \mathrm{ml})$ are displayed as positive control (means $\pm S D, n=3, * p<0,0001)$. Modified from (Menck et al, 2014b).

Since EV have often been described to transfer malignant characteristics to surrounding benign cells, we examined the influence of T-MV on benign hTERT-HME1 cells in microinvasion assays. As shown in Fig. 8E, neither stimulation with autologous hTERT-MV nor heterologous malignant $\mathrm{T}-\mathrm{MV}_{\mathrm{M}}$ was able to induce an invasive phenotype in the cells suggesting that the recipient cell has to be predisposed for an invasive phenotype. 


\subsubsection{Uptake is essential, but not specific for pro-invasive MV}

We then hypothesized that the phenotypic difference between pro-invasive and non-proinvasive MV populations is caused by a differential uptake of the vesicles into their target cells. To address this question, we incubated MCF-7 breast cancer cells with PKH26-labeled MV and assessed MV uptake by fluorescence microscopy (Fig. 9A). Nonetheless, after $24 \mathrm{~h}$ all MV populations had been taken up by the cancer cells, arguing against a differential uptake as discriminating criterion between pro-invasive and non-pro-invasive MV.

In order to investigate if uptake is nonetheless essential for the pro-invasive function of TMV, we tried to inhibit MV uptake through incubation with endocytosis inhibitors which target different endocytotic pathways. On the one hand, we chose filipin III which interferes with caveolae-mediated uptake, on the other hand, we employed dansylcadaverine which blocks clathrin-mediated endocytosis. To titrate the highest tolerable dose of both inhibitors which can be used for treatment without affecting cellular viability, we performed MTT assays (Fig. 9B) as well as proliferation assays with the xCELLigence system (Fig. 9C). Based on known $\mathrm{IC}_{50}$ concentrations in the literature as well as the data from our viability analyses we chose to assess the inhibitory effect of $10 \mu \mathrm{M}$ filipin III as well as $25 \mu \mathrm{M}$ dansylcadaverine on T-MV uptake into MCF-7 cells. However, MV uptake was influenced by neither of the two inhibitors (Fig. 9D) suggesting that MV uptake occurs independent of classical endocytotic routes via clathrin- or caveolin-mediated endocytosis.

Next, we targeted dynamin which is a large membrane protein involved in diverse endocytotic pathways. It is required for the scission, and thereby uptake, of invaginated endocytotic vesicles into the cell (Doherty \& McMahon, 2009; McNiven et al, 2000). Interestingly, dynasore which is a potent inhibitor of the GTPase activity of dynamin was able to reduce TMV uptake into MCF-7 cells by around 50\% in concentrations which did not interfere with cellular viability (Fig. 9E+F). Moreover, pre-incubation of cancer cells with dynasore significantly impaired the pro-invasive effect of T-MV on MCF-7 cells in microinvasion assays (Fig. 9G). These results indicate that MV uptake occurs in a dynamin-dependent mechanism and is an important pre-requisite for MV function. 
A
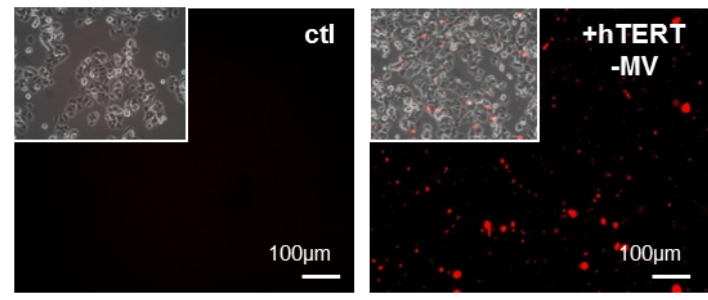

B

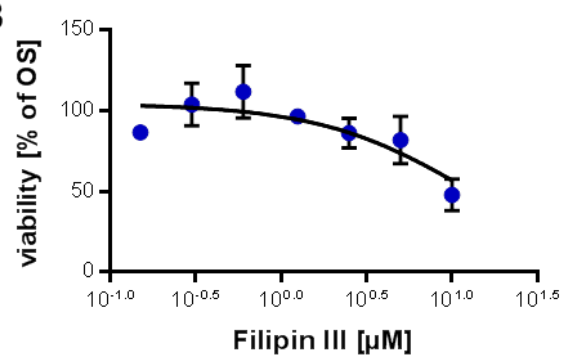

C

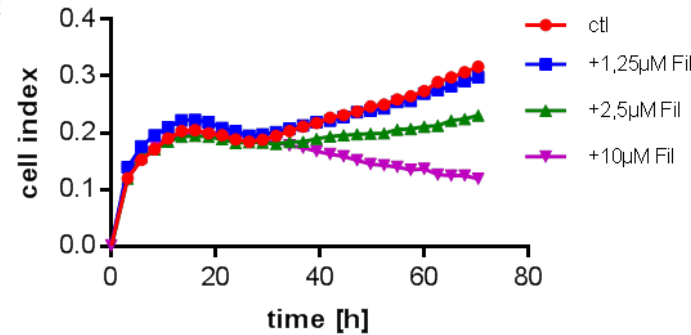

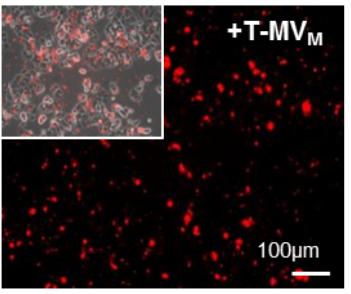
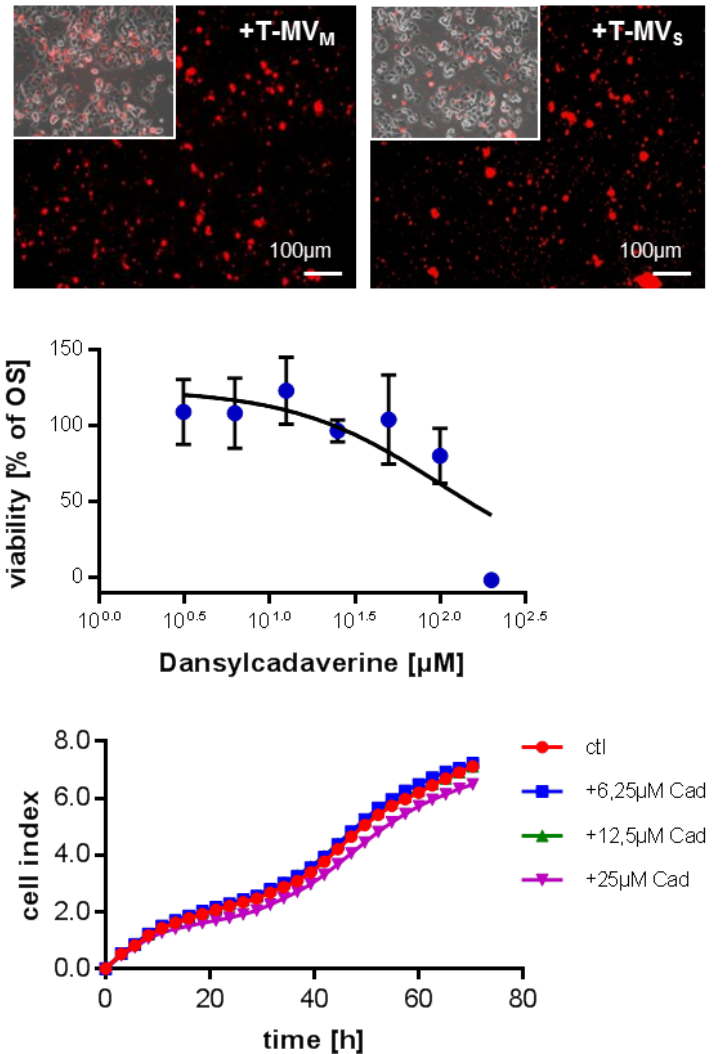

E

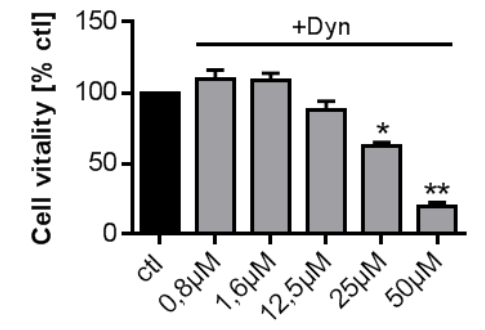

$\mathbf{F}$

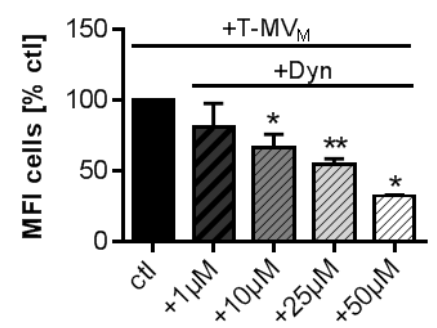

G

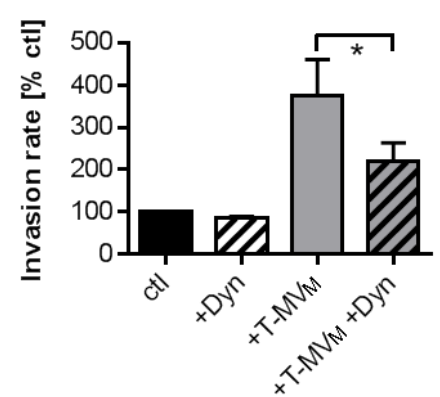

H

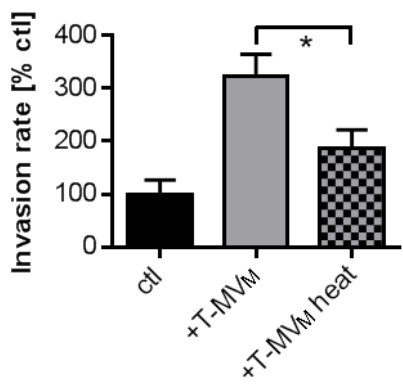

Fig. 9: MV uptake does not differ between MV populations, but is important for their proinvasive function and relies on a dynamin-dependent mechanism

$A$, MCF-7 cells were stimulated with PKH26-labeled MV $(5 \mu \mathrm{g} / \mathrm{ml})$ for $24 \mathrm{~h}$ and uptake was evaluated by fluorescence microscopy (magnification: 10x, inserts: bright fields). $\boldsymbol{B}+\boldsymbol{C}$, MTT assay $(B)$ and xCELLigence measurements $(C)$ of MCF-7 cells treated for $96 \mathrm{~h}$ or $72 \mathrm{~h}$, respectively, with filipin III (Fil) or dansylcadaverine (Cad). OS = Overall survival $\boldsymbol{D}$, Flow cytometry: Treatment of MCF-7 cells with Fil $(10 \mu \mathrm{M})$ or Cad $(25 \mu \mathrm{M})$ for $24 \mathrm{~h}$ has no effect on MV uptake (means $\pm \mathrm{SD}, \mathrm{n}=3$ ). $\boldsymbol{E}$, MTT assay of MCF-7 cells incubated for $48 \mathrm{~h}$ with increasing concentrations of the dynamin-inhibitor dynasore (Dyn) (means $\pm \mathrm{SD}, \mathrm{n}=3,{ }^{*}<00,01, *{ }^{*}<0,001$ ). $\boldsymbol{F}+\boldsymbol{G}$, Treatment with Dyn significantly decreases MV uptake into MCF-7 cells $(F)$ (means $\pm \mathrm{SD}, \mathrm{n}=3,{ }^{*} \mathrm{p}<0,01$, $* * \mathrm{p}<0,001)$ resulting in a reduction of the pro-invasive effect of T-MV on MCF-7 cell invasion in microinvasion assays $(G)$ (means $\left.\pm \mathrm{SD}, \mathrm{n}=3,{ }^{*} \mathrm{p}<0,001\right)$. $\boldsymbol{H}$, Heating of T-MV $\left(5 \mathrm{~min}, 95^{\circ} \mathrm{C}\right)$ significantly reduces the proinvasive potential of T-MV on MCF-7 invasiveness (means $\pm \mathrm{SD}, \mathrm{n}=4,{ }^{*} \mathrm{p}<0,001$ ). Modified from (Menck et al, 2014b). 
Next, we asked whether the pro-invasive effect of T-MV might be caused by proteins which are differentially expressed on malignant and benign MV. Therefore, we heated T-MV for $5 \mathrm{~min}$ at $95^{\circ} \mathrm{C}$ which leads to protein denaturation due to the disruption of hydrogen bonds and non-polar hydrophobic interactions. Heated T-MV induced significantly less tumor invasion compared to non-heated T-MV (Fig. 9H) which points to the fact that parts of the pro-invasive effect of malignant MV are indeed mediated by proteins.

\subsubsection{EMMPRIN - a novel marker for MV}

In order to identify which proteins are differentially expressed on pro-invasive and non-proinvasive MV, we investigated EMMPRIN as a possible candidate protein. Previous experiments from our group had shown that knockdown of EMMPRIN in tumor cells antagonizes the pro-invasive tumor-M $\phi$ crosstalk in co-culture experiments (Hagemann et al, 2005). Western Blot analyses revealed an enrichment of EMMPRIN on T-MV, whereas it was absent on T-Exo (Fig. 10A+B). Since EMMPRIN can be glycosylated at different sites, it can be found in two different glycoforms: Either in its highly glycosylated form (HG EMMPRIN, 45-65 kDa) or in a less glycosylated form (LG EMMPRIN, $32 \mathrm{kDa}$ ). Both forms were found on T-MV, although LG-EMMPRIN was only weakly expressed compared to HG-EMMPRIN.

In order to evaluate EMMPRIN as a novel marker for MV, we screened EV from both breast cancer cell lines, MCF-7 and SK-BR-3, for the expression of known MV and Exo markers (Fig. 10C). The established Exo marker Tsg101 was indeed predominantly present on Exo, as was the transcription factor Runx1. In contrast, $\alpha$-tubulin was mainly found at high levels in MV. Surprisingly, Flotillin-2 which is often used as marker for Exo was expressed on MV as well as Exo and is therefore in our hands no suitable marker for the discrimination of both. We additionally investigated these established markers for discrimination of MV and Exo in sucrose gradient preparations. Exo usually float in density fractions ranging from 1,13$1,19 \mathrm{~g} / \mathrm{ml}^{-1}$ (Thery et al, 2006) and sucrose gradient ultracentrifugation is often recommended as the standard method to separate Exo from MV (Gyorgy et al, 2011b).

$\mathrm{T}-\mathrm{MV}_{\mathrm{S}}$ and $\mathrm{T}-\mathrm{Exo}_{\mathrm{S}}$ were layered separately on sucrose step gradients. The distribution of the markers was analyzed by Western Blotting (Fig. 10D). Interestingly, both vesicle populations floated in comparable density fractions ranging from 1,13-1,22 $\mathrm{g} / \mathrm{ml}^{-1}$. Compared to the other markers, EMMPRIN was the only protein which was almost exclusively found on MV, while the others were present to some extent on both EV populations. Furthermore, the results 
suggest that sucrose gradient floatation alone is no suitable method for the accurate separation of MV and Exo in the same solution.

A

$\frac{M C F-7}{C \quad M V} \frac{\text { SK-BR-3 }}{C \quad M V}$

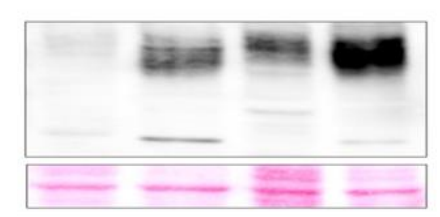

HG EMP (45-65kDa)

LG EMP (32kDa)

Ponceau

C
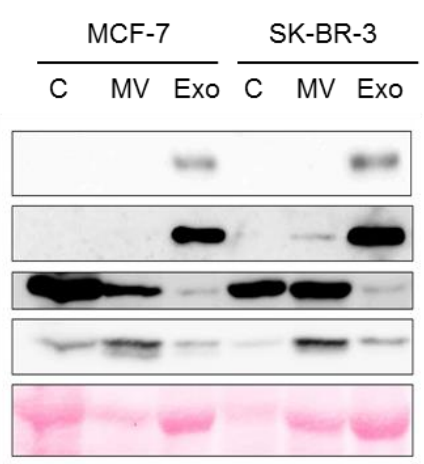
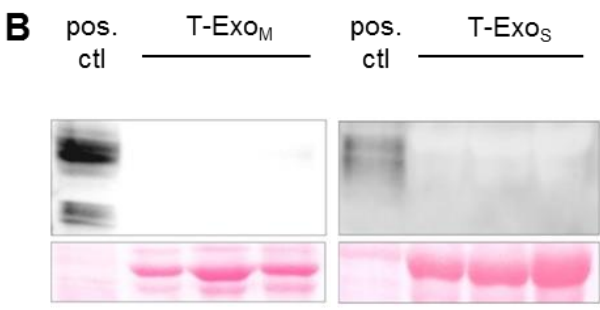

HG EMP

LG EMP

Ponceau

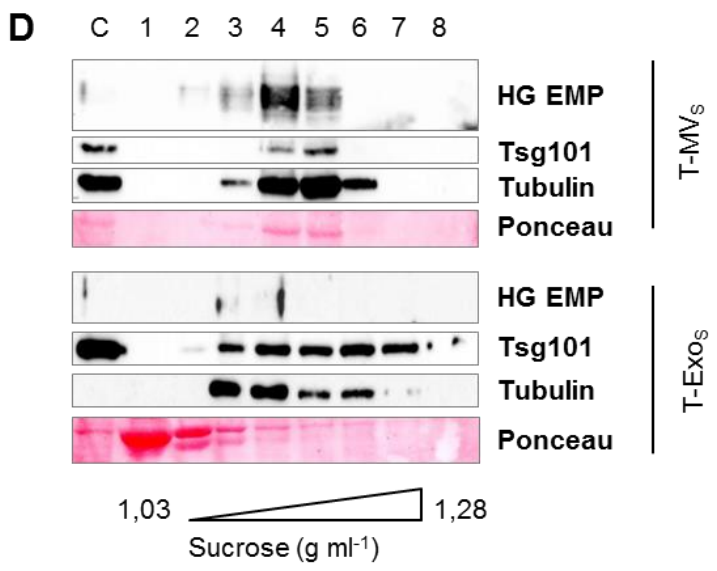

Fig. 10: EMMPRIN expression discriminates MV and Exo

$\boldsymbol{A}+\boldsymbol{B}$, Western Blot analysis of EMMPRIN (EMP) expression on MV (A) and Exo (B) from MCF-7 and SK-BR3 cells showing an enrichment on T-MV, while it is absent on T-Exo. Due to differential glycosylation EMMPRIN can occur in a highly glycosylated (HG EMP) or low glycosylated (LG EMP) form. C $=$ Whole cell lysates; $\boldsymbol{C}$, Comparative analysis of known MV and Exo markers (Western Blot). $\boldsymbol{D}, \mathrm{T}-\mathrm{MV}_{\mathrm{S}}$ and $\mathrm{T}$-Exos were loaded separately on sucrose step gradients and the distribution of the vesicles in the eight different density fractions was analyzed based on the established MV and Exo markers. Modified from (Menck et al, 2014b).

\subsubsection{EMMPRIN is involved in the pro-invasive function of T-MV}

After having established EMMPRIN as a sensitive and specific marker which is expressed at high levels on T-MV, we asked if it is critical for the pro-invasive phenotype of T-MV. Therefore, we performed a stable knockdown of EMMPRIN via shRNA (see 2.2.1.2) in SKBR-3 cells because their MV showed the highest endogenous EMMPRIN expression (Fig. $10 \mathrm{~A})$.

After knockdown, EMMPRIN was only weakly detectable in the cells and their released MV (Fig. 11A). Consistently, this resulted in a significantly impaired pro-invasive effect of T$\mathrm{MV}_{\mathrm{S}}$ from EMMPRIN-knockdown (shEMP) cells compared to $\mathrm{T}-\mathrm{MV}_{\mathrm{S}}$ from cells transfected with an unspecific non-sense control shRNA (ns ctl) (Fig. 11B+C). These results point towards EMMPRIN as one of the factors which mediate the pro-invasive phenotype of T-MV. Since we had shown that ingestion of T-MV is important for their pro-invasive effect, we 
analyzed whether EMMPRIN knockdown interferes with this process. However, there was no difference in the uptake of $\mathrm{T}-\mathrm{MV}_{\mathrm{S}}$ from shEMP or ns ctl cells into MCF-7 or SK-BR-3 breast cancer cells (Fig. 11D+E).
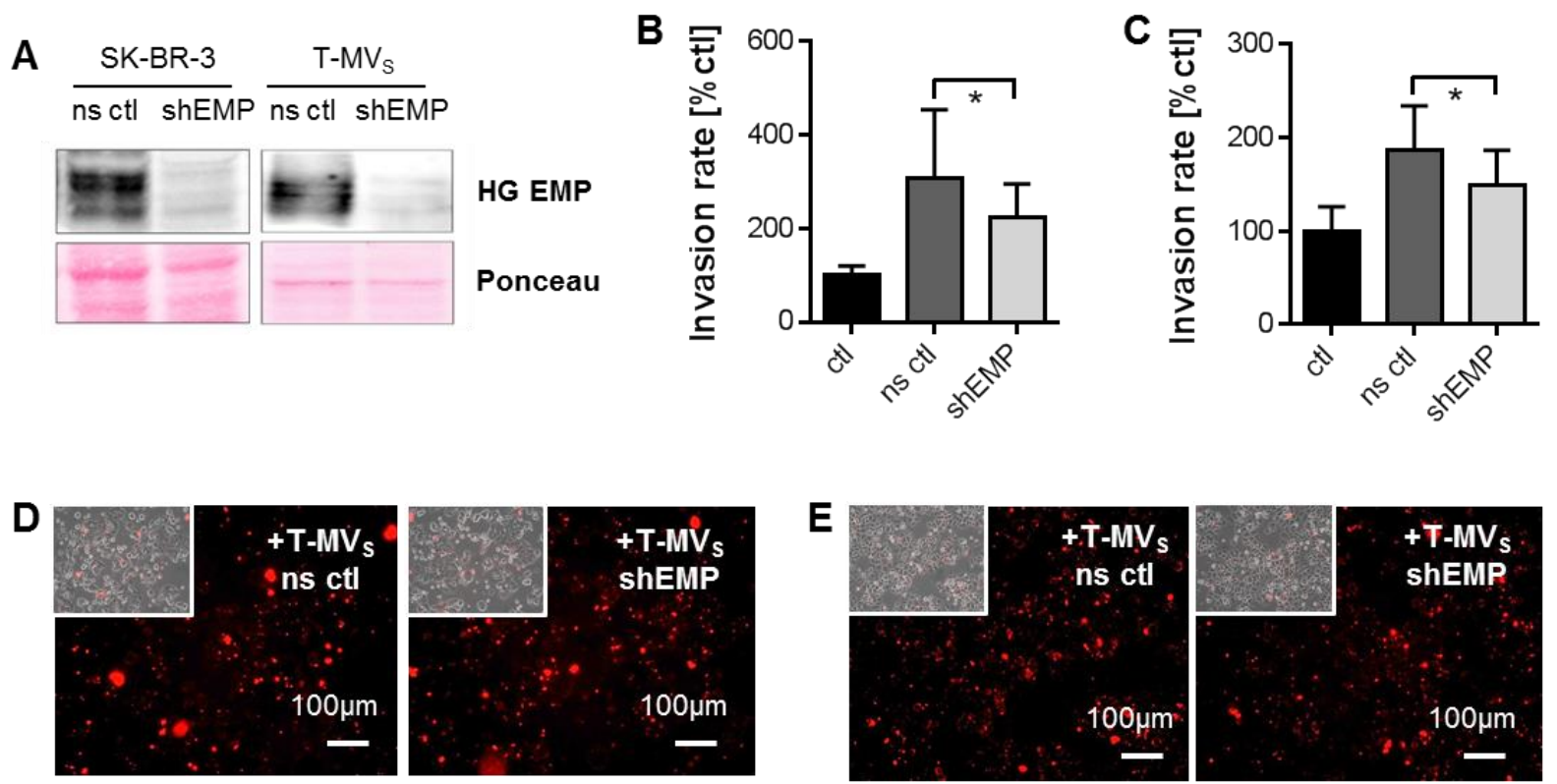

Fig. 11: Knockdown of EMMPRIN in SK-BR-3 cells reduces the pro-invasive effect of T-MV

$\boldsymbol{A}$, Western Blot: Comparison of the EMMPRIN expression on cells and corresponding MV from SK-BR-3 cells stably transfected with a non-sense shRNA (ns ctl) or an EMMPRIN-specific shRNA (shEMP). $\boldsymbol{B}+\boldsymbol{C}$, The proinvasive effect of T-MV $\mathrm{S}(1 \mu \mathrm{g} / \mathrm{ml})$ from SK-BR-3 shEMP cells on MCF-7 $(B)$ and SK-BR-3 $(C)$ cell invasion was significantly reduced compared to MV from SK-BR-3 ns ctl cells (microinvasion assays, means $\pm \mathrm{SD}, \mathrm{n}=3$, $\left.{ }^{*} \mathrm{p}<0,01\right)$. $\boldsymbol{D}+\boldsymbol{E}$, There was no significant difference in the uptake of PKH26-labeled T-MV $\mathrm{V}_{\mathrm{S}}$ from shEMP or ns ctl cells into MCF-7 $(D)$ or SK-BR-3 $(E)$ cells as visualized by fluorescence microscopy (magnification: 10x, inserts: bright fields). Modified from (Menck et al, 2014b).

\subsubsection{The glycosylation status of EMMPRIN is important for its function}

Since the experiments identified EMMPRIN as one of the pro-invasive factors on T-MV, we then asked whether differential expression on malignant and benign MV would explain their different function (Fig. 12A). Surprisingly, the benign hTERT-HME1 displayed the strongest EMMPRIN expression in Western Blot analyses indicating that EMMPRIN expression per se does not discriminate malignant and benign MV. However, while all pro-invasive T-MV seemed to express a very highly glycosylated form of EMMPRIN (HG-EMMPRIN, $>50 \mathrm{kDa}$ ), benign P-MV and hTERT-MV both expressed a less glycosylated form which has been undescribed so far and which we termed intermediately glycosylated EMMPRIN (IGEMMPRIN, 45-50 kDa). 
A

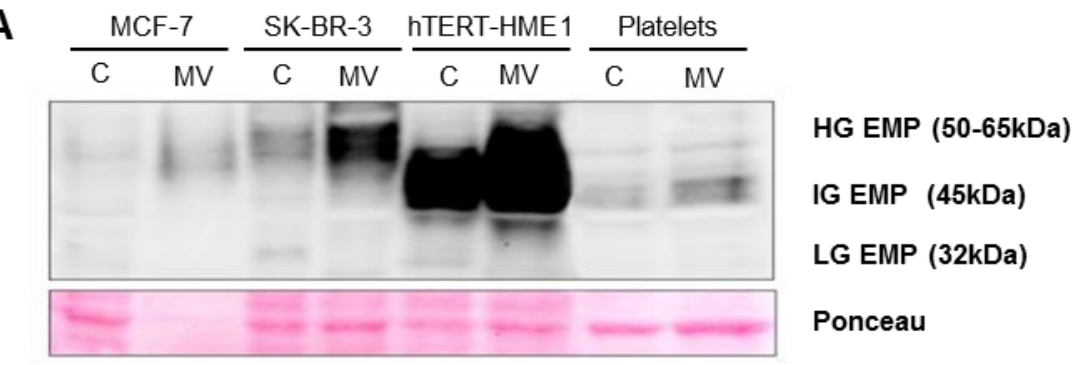

B

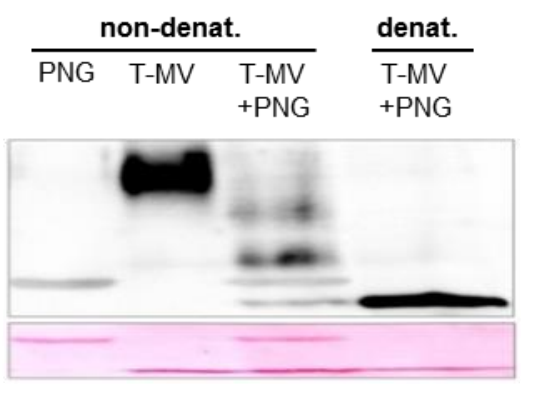

HG EMP

IG EMP

LG EMP

Core protein

Ponceau

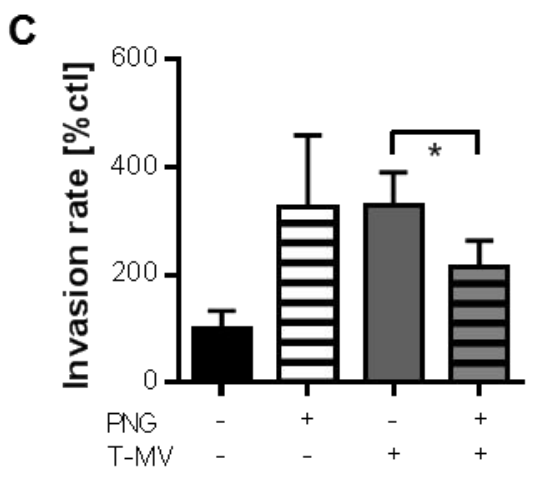

Fig. 12: The pro-invasive effect of T-MV is mediated by HG-, and not IG-EMMPRIN

$A$, Western Blot: Expression of EMMPRIN was analyzed in various cells $(=C)$ and their corresponding MV. While all pro-invasive MV carry a very highly glycosylated form of EMMPRIN (HG-EMP, $>50 \mathrm{kDa}$ ), all benign MV express an only intermediately glycosylated form (IG-EMP, 45-50 kDa). B, Deglycosylation of T-MV under non-denaturing conditions with PNGaseF $\left(1 \mathrm{~h}, 37^{\circ} \mathrm{C}\right.$, visible as unspecific band in lane $\left.1+3\right)$ results in a loss of HG-EMP and reveals the less glycosylated IG- and LG-EMP. As positive control T-MV $\mathrm{T}_{\mathrm{M}}$ were deglycosylated under denaturing conditions which leads to full deglycosylation to the core protein. $\boldsymbol{C}$, Microinvasion assay: $\mathrm{T}^{-} \mathrm{MV}_{\mathrm{M}}(10 \mu \mathrm{g} / \mathrm{ml})$ deglycosylated under non-denaturing conditions induce significantly

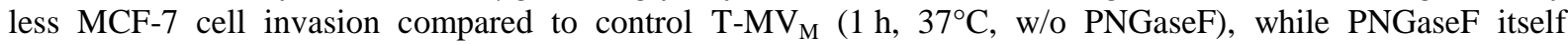
stimulates tumor invasion (means $\pm \mathrm{SD}, \mathrm{n}=3,{ }^{*} \mathrm{p}<0,001$ ). Taken from (Menck et al, 2014b).

To confirm that HG- and IG-EMP are indeed two different glycoforms, we deglycosylated T$\mathrm{MV}_{\mathrm{M}}$ with Peptide-N-Glyosidase $\mathrm{F}$ (PNGaseF) which cleaves between asparagine residues and $\mathrm{N}$-acetylglucosamines and thereby specifically releases $\mathrm{N}$-linked oligosaccharides (Maley et al, 1989). Deglycosylation under non-denaturing conditions for $1 \mathrm{~h}$ at $37^{\circ} \mathrm{C}$ led to only partial deglycosylation with a loss of HG-EMMPRIN which was converted to less glycosylated IG- and LG-EMMPRIN (Fig. 12B). Complete deglycosylation to the core protein $(27 \mathrm{kDa})$ was only observed when $\mathrm{T}-\mathrm{MV}_{\mathrm{M}}$ were denatured $\left(10 \mathrm{~min}, 100^{\circ} \mathrm{C}\right)$ prior to incubation with PNGaseF in corresponding G7 reaction buffer. To test whether T-MV carrying IG- and LG-EMMPRIN instead of HG-EMMPRIN are still able to induce tumor invasion, we stimulated MCF-7 cells in microinvasion assays with $\mathrm{T}-\mathrm{MV}_{\mathrm{M}}$ which had been deglycosylated under non-denaturing conditions (Fig. 12C). Interestingly, these T-MV had a significantly less pronounced effect on tumor invasion compared to control T-MV. This clearly suggests that especially HG-EMMPRIN is involved in the pro-invasive MV function. To exclude that the diminished tumor cell invasiveness is caused due to a direct effect of 
residual PNGaseF activity on the tumor cells, we additionally stimulated MCF-7 cells with the enzyme alone. On the contrary, this increased tumor invasion (Fig. 12C) which shows that there are indeed additional effects due to deglycosylation of other proteins on the tumor cells, however, they rather counteract the observed effect of deglycosylated T-MV.

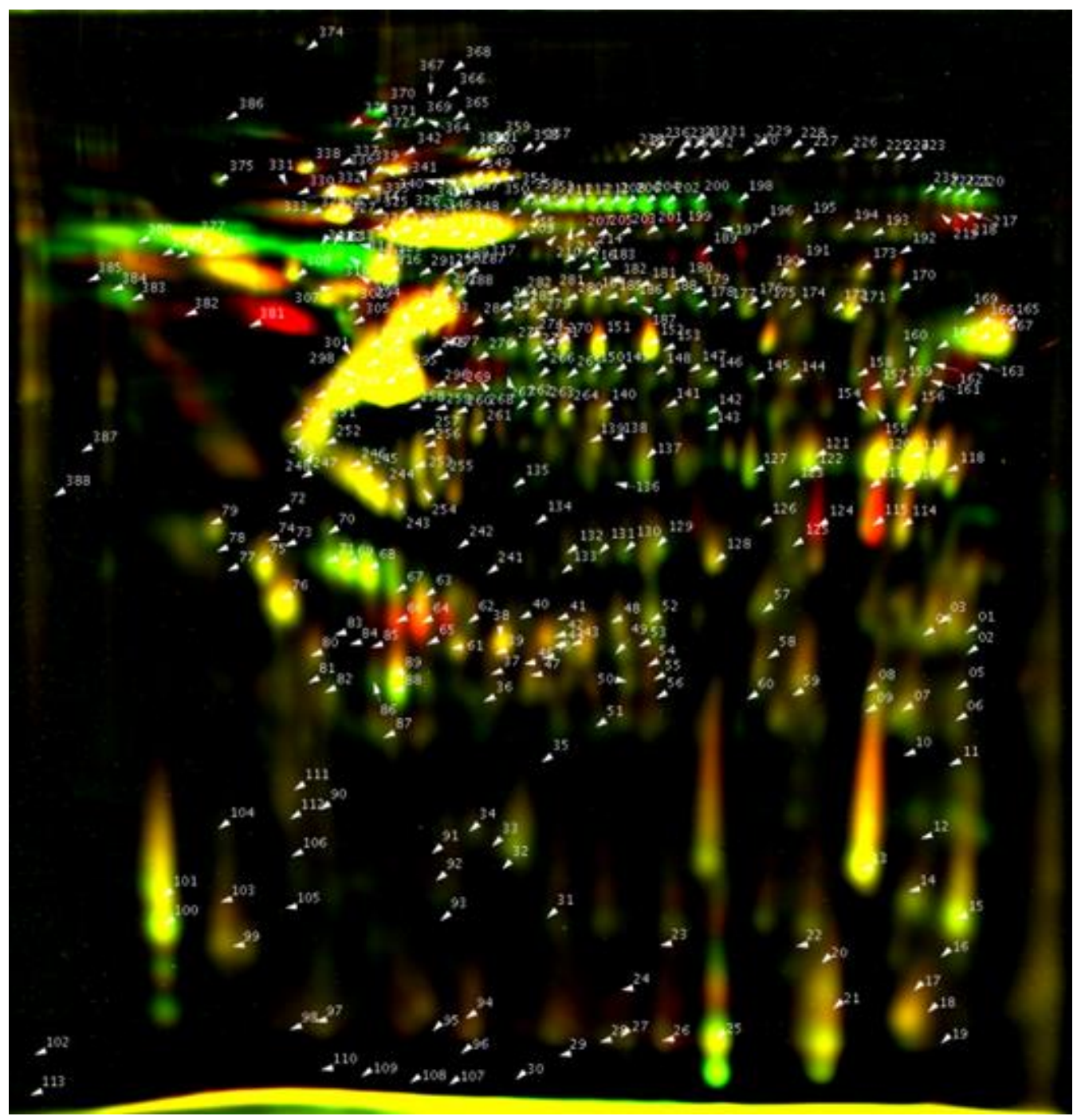

Fig. 13: 2D-DIGE of deglycosylated T-MV

This image represents an overlay of two separately scanned images from a 2D-DIGE gel of untreated versus deglycosylated (non-denaturing conditions) $\mathrm{T}^{-\mathrm{MV}_{\mathrm{M}}}(30 \mu \mathrm{g}$ each). Protein spots were numbered, manually excised and subjected to mass spectrometry. Green = proteins with stronger expression on untreated T-MV $\mathrm{M}_{\mathrm{M}}$; Red = proteins with stronger expression on $\mathrm{T}^{-\mathrm{MV}_{\mathrm{M}}}$ after deglycosylation with PNGaseF; Yellow = proteins equally expressed in both samples.

Since PNGaseF deglycosylates all N-glycosylated proteins on T-MV, it is possible that other proteins contribute to the observed reduction in MV-mediated tumor invasion. In order to investigate whether and which other glycoproteins on $\mathrm{T}-\mathrm{MV}_{\mathrm{M}}$ might be affected by removal of N-linked oligosaccharides, we performed 2D-DIGE from untreated versus PNGaseFtreated $\mathrm{T}_{-} \mathrm{MV}_{\mathrm{M}}$ in cooperation with Kathrin Darm and Dr. Christian Scharf (University 
Medicine Greifswald). In first experiments a good separation of the MV proteins was achieved and 388 protein spots were identified, excised and subjected to mass spectrometry for protein identification (Fig. 13). Glycoproteins which are affected by PNGaseF treatment are visible as green or red spots on the gel, depending on the fact in which sample (untreated or PNGaseF-treated) they show a stronger expression. Yellow spots represent an equal expression of the protein under both conditions. All spots which could be identified by mass spectrometry exclusively in either condition (marked in red/green) are listed in chapter 7.1. Unfortunately, not all picked spots, including EMMPRIN, could be positively matched to a corresponding protein, probably due to the low abundance of these proteins which was not sufficient for mass spectrometry identification.

\subsubsection{Identification of a novel EMMPRIN glycoform}

To further confirm our hypothesis that HG- and IG-EMMPRIN are indeed characterized by a differential glycosylation pattern on $\mathrm{T}-\mathrm{MV}$, we deglycosylated $\mathrm{T}-\mathrm{MV}_{\mathrm{M}}$ under non-denaturing conditions with PNGaseF and subjected them to SDS-PAGE for separation of the different EMMPRIN glycoforms. All EMMPRIN-containing bands identified by immunoblotting (see Fig. 12B) were manually excised from the gel and sent to Greifswald for LC-MS/MS analyses. Mass spectrometry and data analysis were performed by Dr. Christian Scharf and are described in detail in (Menck et al, 2014b).

All peptides identified by mass spectrometry were aligned with the four known EMMPRINisoforms to assess sequence coverage (Fig. 14A). As expected, neither the signal peptide (121) nor peptides from the transmembrane domain (324-344) were found in any sample. Moreover, peptides specific for the isoforms EMMPRIN-1 or EMMPRIN-4 were also missing. Spectral counting of the N-terminal fragments of EMMPRIN-2 and -3 presented a ratio of 10:1 which confirms that EMMPRIN-2 is the most abundant isoform on T-MV $\mathrm{M}_{\mathrm{M}}$. To address the question if EMMPRIN-2 is also involved in tumor progression in vivo, we performed an mRNA screening on brain metastases from eight breast cancer patients and compared them to our in vitro breast (cancer) cell lines. Indeed, EMMPRIN-2 was expressed in the tumor tissues and samples as well as in the benign cell line hTERT-HME1 (Fig. 14B) which further supports the hypothesis that not EMMPRIN expression per se, but posttranslational modifications are essential for its function. 

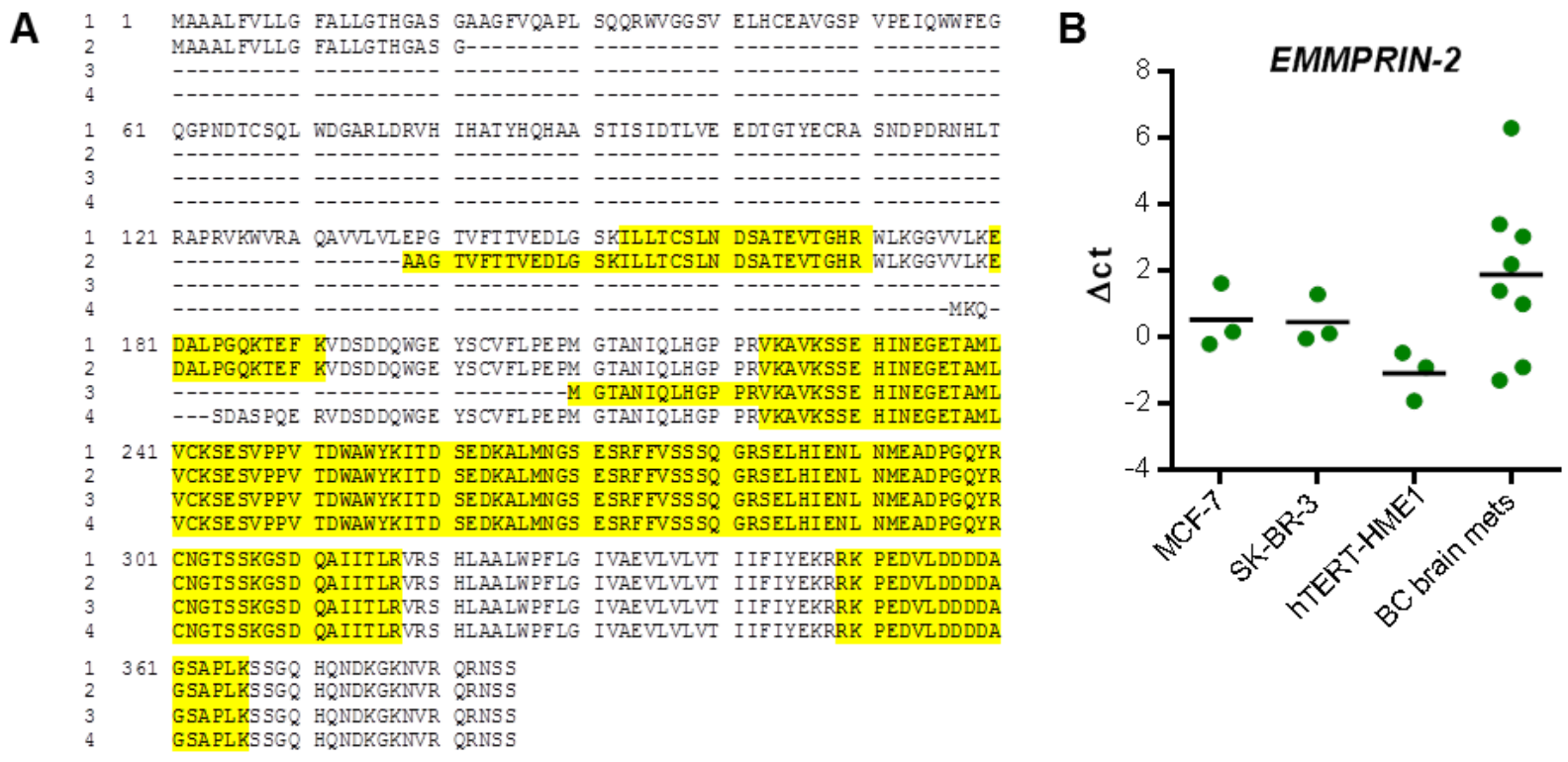

Fig. 14: EMMPRIN-2 is the predominant isoform on $T-M V_{M}$

$A$, Sequence coverage of the identified peptides (yellow) in LC-MS/MS analyses for the four known EMMPRIN isoforms (1-4). $\boldsymbol{B}$, The expression of EMMPRIN-2 was assessed by qRT-PCR from total RNA samples of the two breast cancer cell lines MCF-7 and SK-BR-3, benign hTERT-HME1 mammary epithelial cells as well as eight brain metastases from breast cancer patients (BC brain mets). Modified from (Menck et al, 2014b).

Mass spectrometry was further used to analyze the glycosylation status of the three predicted $\mathrm{N}$-glycosylation sites of EMMPRIN which are displayed in Fig. 15A. Because of their large size, glycosylated peptides cannot be detected by mass spectrometry. In contrast, when Nlinked glucosaccharides are removed from their asparagine $(=\mathrm{N})$ residues by PNGaseF treatment, this causes deamidation of the N-residues to aspartic acid (=D) and allows the detection of these fragments which are characterized by an increase in their peptide mass by 1 mass unit. Therefore, absence of a peptide with a putative N-glycosylation site which reappears after deglycosylation of the samples with a deamidated N-residue, identifies $\mathrm{N}$ glycosylation sites.

Based on this principle, we screened all three predicted N-glycosylation sites. Indeed, the two peptides which carry the glycosylation site N160 or N268, respectively, were absent in HGEMMPRIN, while they were detected with deamidated N-residues in IG-EMMPRIN and core protein samples (Fig. 15B-D, Fig. 47). Additionally, they were also found in the HGEMMPRIN spot after subsequent in-gel deglycosylation with PNGaseF. There was no consistent deamidation pattern detectable for the third predicted glycosylation site at N302. Taken together, these results show that HG-EMMPRIN on the pro-invasive $T-\mathrm{MV}_{M}$ is $\mathrm{N}$ glycosylated at the residues N160 and N268, while they are not glycosylated in the IGEMMPRIN form which is expressed on non-pro-invasive MV. 
A

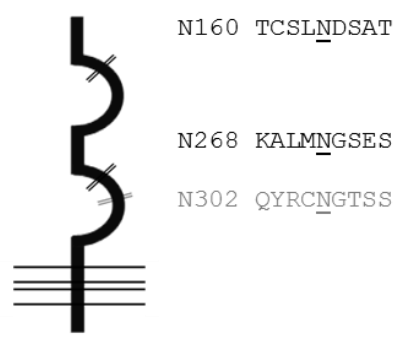

B

HG

IG

HG

IG

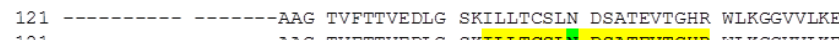
121 121 ----------AAG TVFTTVEDLG SKILLTCSLN DSATEVTGHR WLRGGVVLKE

241 VCKSESVPPV TDWAWYKITD SEDKALMNGS ESRFFVSSSQ GRSELHIENL NMEADPGQYR 241 VCKSESVPPV TDWAWYKITD SEDKALMNGS ESRFFVSSSQ GRSELHIENL NMEADPGQYA 241 VCKSESVPPV TDWAWYKITD SEDKALMNGS ESRFFVSSSQ GRSELHIENL NMEADPGQYP
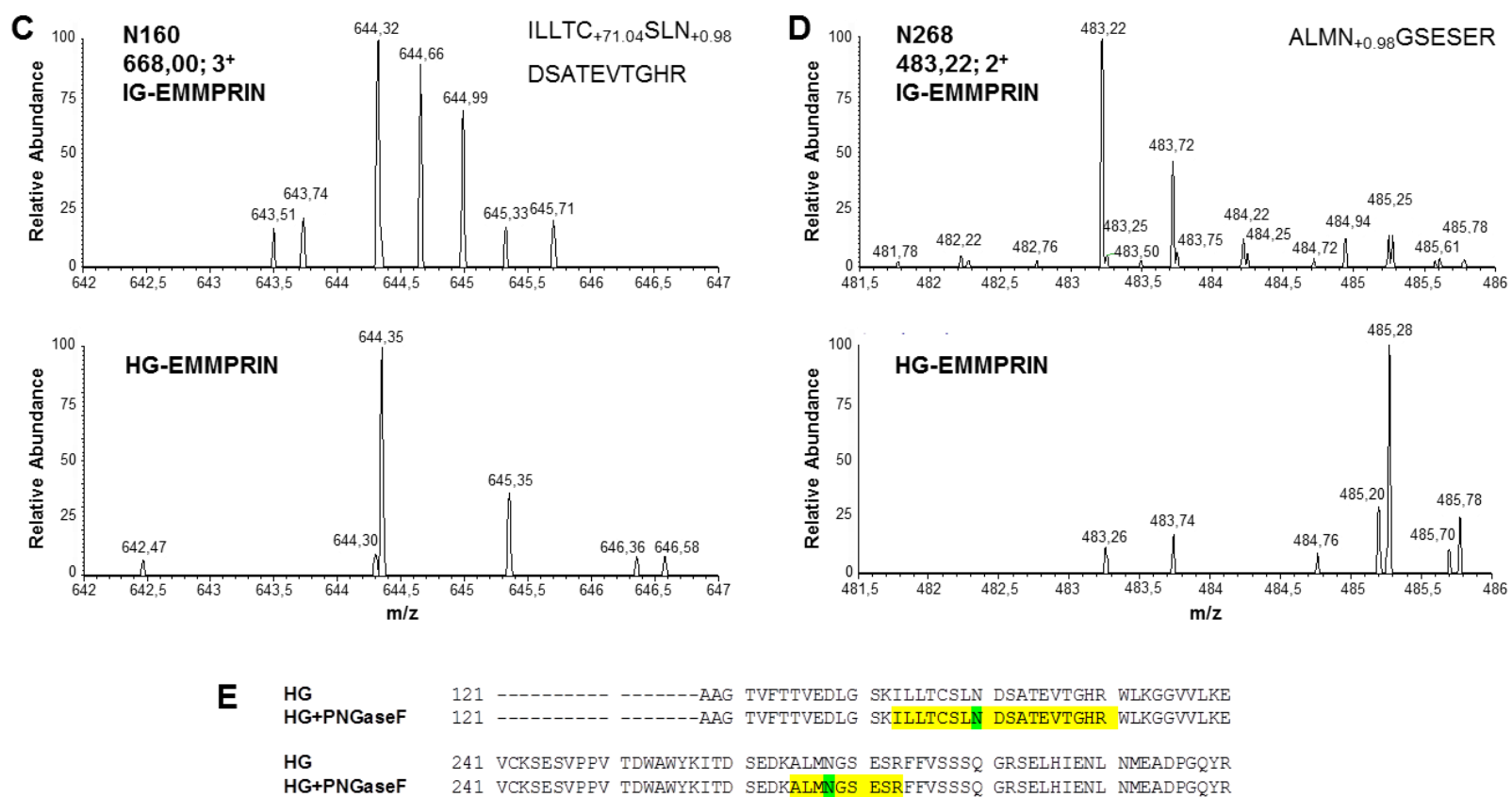

Fig. 15: Mass spectrometry reveals differential glycosylation of HG- and IG-EMMPRIN

$\boldsymbol{A}$, Schematic representation of EMMPRIN-2 with the three known N-glycosylation sites. $\boldsymbol{B}$, While the peptides with the two N-glycosylation sites N160 and N268 (yellow) are absent in HG-EMMPRIN, they are detectable in IG-EMMPRIN as well as core protein samples with deamidated N-residues (green). $\boldsymbol{C}+\boldsymbol{D}$, MS spectra for the two peptides carrying N160 or N268, respectively, which were not present in HG-EMMPRIN spots. $\boldsymbol{E}$, In-gel deglycosylation of the HG-EMMPRIN spot with PNGaseF led to detection of both peptides with deamidated Nresidues which confirms glycosylation at N160 and N268 in HG-EMMPRIN. Modified from (Menck et al, 2014b).

\subsubsection{EMMPRIN-carrying T-MV increase tumor invasion through activation of p38/MAPK}

Next, we were interested in the mechanism which mediates MV-induced tumor invasion. Since pro-invasive T-MV we found to express HG-EMMPRIN at high levels and the role of MV-bound EMMPRIN for induction of MMP is well established (Muralidharan-Chari et al, 2010), we first investigated MMP levels in MCF-7 cells after stimulation with T-MV $\mathrm{M}_{\mathrm{M}}$. However, $\mathrm{T}-\mathrm{MV}_{\mathrm{M}}$ did not augment the expression of selected MMP including MMP14, MMP15 or MMP9 (Fig. 16A). Moreover, also the expression of MMP-9 and MMP-2 remained unchanged in zymography assays of the supernatant of MCF-7 cells after T-MV stimulation (Fig. 16B). Only P-MV which were employed as negative control seemed to 
induce a slight induction of MMP-9 on the mRNA and protein level, however, this did not reach statistical significance. Although T-MV have been previously described as carriers for MMP (Dolo et al, 1998; Ginestra et al, 1997), neither $\mathrm{T}^{-} \mathrm{MV}_{\mathrm{M}}$ nor $\mathrm{T}-\mathrm{MV}_{\mathrm{S}}$ were found to express MMP-2 (data not shown) or MMP-9 in zymography assays (Fig. 16C). In contrast, benign MV from unstimulated human M $\phi(\mathrm{M} \phi-\mathrm{MV})$ or P-MV were positive for MMP-9, suggesting that the presence of MMP is not essential for the conveyance of a pro-invasive phenotype of MV. We then looked for the activation of other known pro-invasive EMMPRIN target genes in MCF-7 cells after stimulation with EMMPRIN-bearing T-MV $\mathrm{M}_{\mathrm{M}}$. However, neither VEGFA, CSF1 nor TNF $\alpha$ expression was increased in the cells in qRT-PCR screenings (Fig. 16D). Moreover, EMMPRIN is known to induce its own transcription via a positive feedback loop mechanism. Nonetheless, we also could not observe such autologous stimulation of EMMPRIN expression itself (Fig. 16D).

A

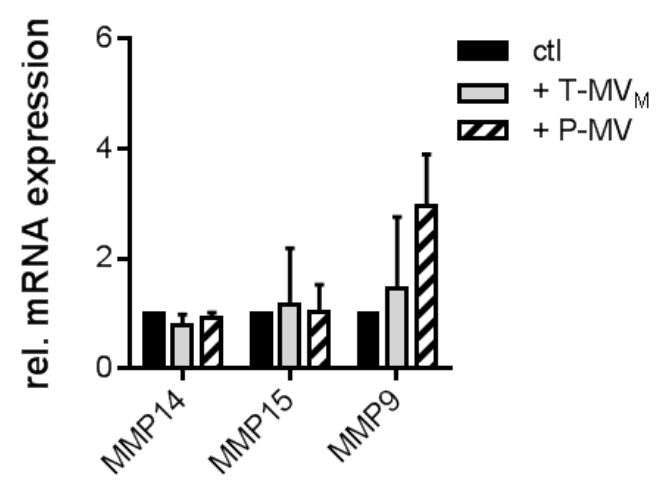

C

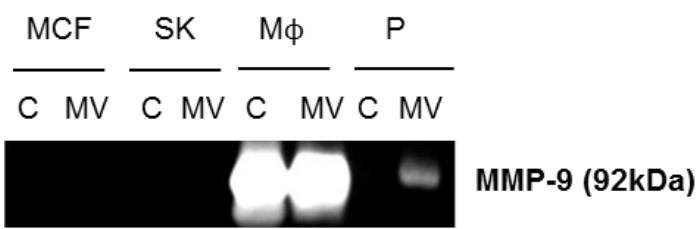

B
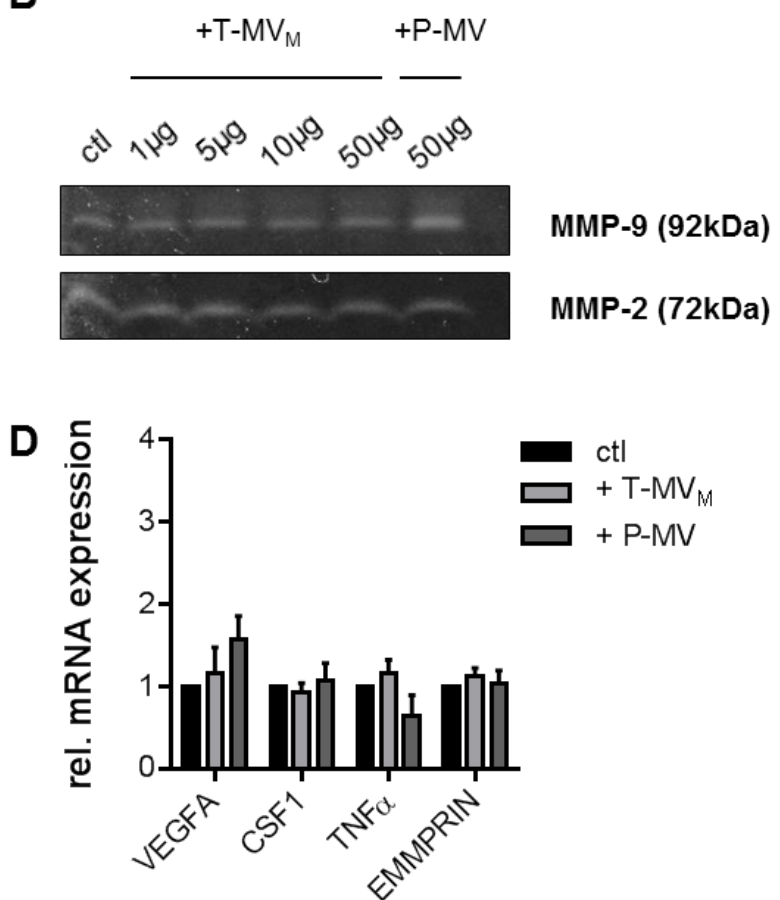

Fig. 16: T-MV have no effect on known EMMPRIN target genes in tumor cells

$\boldsymbol{A}$, The expression of selected MMP was measured by qRT-PCR from total RNA samples of MCF-7 cells stimulated for $24 \mathrm{~h}$ with T-MV $\mathrm{M}_{\mathrm{M}}$ or P-MV (both $25 \mu \mathrm{g} / \mathrm{ml}$ ) (means $\pm \mathrm{SD}, \mathrm{n}=3$ ). $\boldsymbol{B}$, MCF-7 cells were stimulated for $24 \mathrm{~h}$ with increasing concentrations of $\mathrm{T}_{-} \mathrm{MV}_{\mathrm{M}}$ or P-MV and MMP-2 and -9 levels were assessed in tumor cell supernatants by zymography assays. $\boldsymbol{C}$, Comparative analysis of MMP-9 expression in whole cell lysates (=C) and their corresponding MV (zymography). MCF: MCF-7, SK: SK-BR-3, P: platelets. $\boldsymbol{D}$, No changes in mRNA levels of known EMMPRIN target genes were detected in MCF-7 cells after $24 \mathrm{~h}$ of stimulation with T$\mathrm{MV}_{\mathrm{M}}$ or P-MV (both $25 \mu \mathrm{g} / \mathrm{ml}$ ) by qRT-PCR from total RNA samples (means $\pm \mathrm{SD}, \mathrm{n}=3$ ). Modified from (Menck et al, 2014b).

For this reason, we decided to investigated signaling events upstream of target gene induction. One signaling pathway that has been described to participate in EMMPRIN-mediated 
signaling is the p38 (MAPK14) pathway (Lim et al, 1998). Indeed, in time course analyses of p38 pathway activation in MCF-7 cells stimulated with $\mathrm{T}-\mathrm{MV}_{\mathrm{M}}$, we observed a phosphorylation, and thus activation, of p38 after $1 \mathrm{~h}$ of stimulation, while total p38 protein levels remained unchanged (Fig. 17A). To test if the activation of the p38 signaling cascade is indeed involved in MV-mediated tumor invasion, we pre-incubated MCF-7 cells for $2 \mathrm{~h}$ with the pyridinylimidazole compound SB-203580 which binds to the ATP-binding pocket of p38 and thereby prevents its catalytic activity (Kumar et al, 1999). After treatment of the cells with SB-203580 in concentrations which did not interefere with cellular viability (Fig. 17B), T-MV were added and found to induce significantly less tumor invasion in microinvasion assays (Fig. 17C). This indicates that p38 signaling is in fact important for T-MV to confer their autologous pro-invasive effect on tumor cell invasion.

A

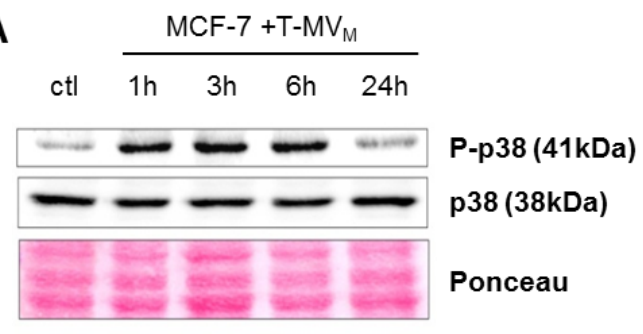

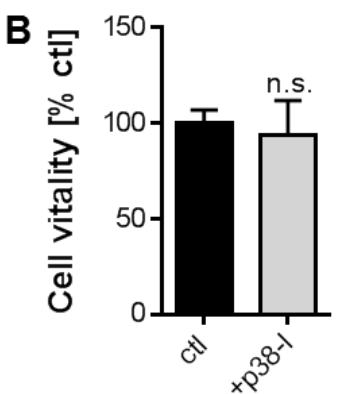

Fig. 17: p38 signaling partially mediates the pro-invasive function of T-MV

$\boldsymbol{A}$, MCF-7 cells were stimulated with $\mathrm{T}-\mathrm{MV}_{\mathrm{M}}(25 \mu \mathrm{g} / \mathrm{ml})$ and expression of $\mathrm{p} 38$ and phosphorylated (P)-p38 was assessed by Western Blotting after different time points. B, MTT assay of MCF-7 cells treated for $96 \mathrm{~h}$ with SB$203580(\mathrm{SB}, 0,5 \mu \mathrm{M})$. n.s. = not significant $\boldsymbol{C}$, Microinvasion assay of MCF-7 cells pre-treated for $2 \mathrm{~h}$ with SB203580 before addition of $10 \mu \mathrm{g} / \mathrm{ml} \mathrm{T}-\mathrm{MV}_{\mathrm{M}}$ (means $\pm \mathrm{SD}, \mathrm{n}=3$, ${ }^{*} \mathrm{p}<0,001$ ). Modified from (Menck et al, 2014b).

Stimulation of MCF-7 cells with T-MV $\mathrm{M}_{\mathrm{M}}$ further induced an increase in phosphorylated c-jun which suggests an additional activation of the JNK-pathway (Fig. 18A). However, in parallel, we observed a concordant augmentation of total c-jun protein levels (Fig. 18A). This raised the question if the observed increase might be caused due to enhanced $J U N$ transcription and thus synthesis of the c-jun protein. Therefore, we performed qRT-PCR analyses of MCF-7 cells which had been stimulated with $T-\mathrm{MV}_{\mathrm{M}}$ revealing that there was no increase, but, in contrast, a slight decrease in JUN mRNA levels (Fig. 18B). Since MV have often been demonstrated to function as vehicles for the transfer of proteins, we investigated whether this is also the case for the c-jun protein. Using Western Blots, we were indeed able to detect c-jun inside $\mathrm{T}-\mathrm{MV}_{\mathrm{M}}$ (Fig. 18C) which suggests its transfer to the tumor cells via MV and might represent a possible explanation for the increased c-jun levels in MCF-7 after T-MV 
stimulation. Nonetheless, inhibition of JNK signaling with a specific JNK inhibitor did not antagonize T-MV-induced tumor invasion (Fig. 18D) arguing against an involvement of JNKsignaling in the conveyance of the autologous pro-invasive effect of T-MV.

A

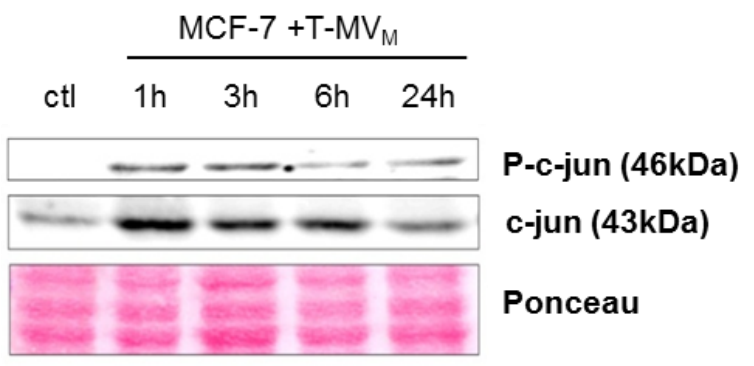

C

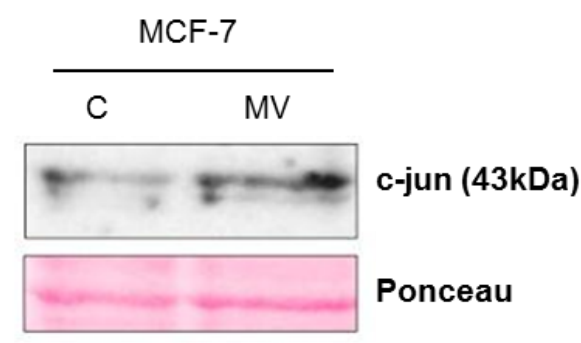

B
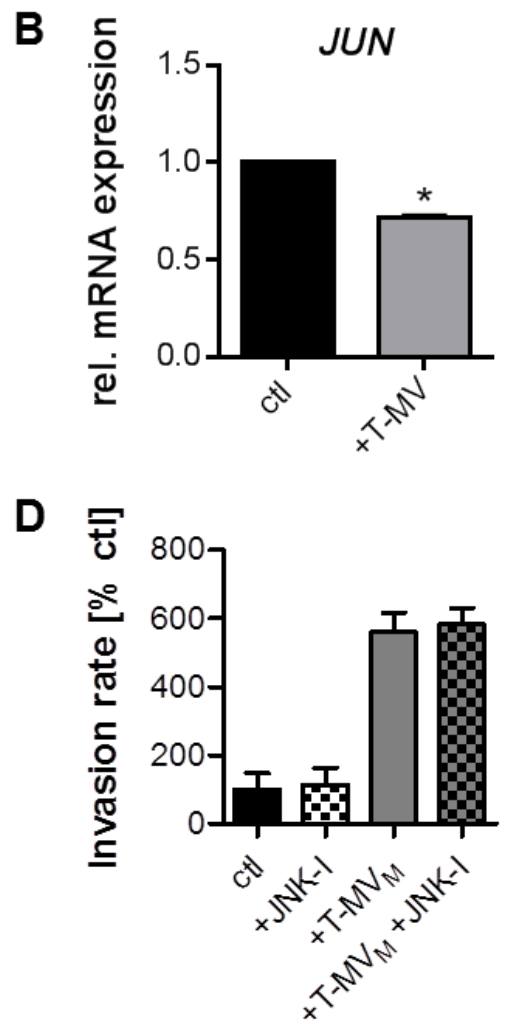

Fig. 18: JNK-signaling is not involved in the pro-invasive function of T-MV

$\boldsymbol{A}$, Western Blot: MCF-7 were stimulated with $\mathrm{T}_{-\mathrm{MV}}(25 \mu \mathrm{g} / \mathrm{ml})$ and the cells harvested after the indicated time points to examine the expression of phosphorylated (P-) c-jun and total c-jun. $\boldsymbol{B}$, Expression of JUN was slightly reduced in MCF-7 cells after $24 \mathrm{~h}$ of stimulation with T-MV $\mathrm{M}(25 \mu \mathrm{g} / \mathrm{ml})$ in qRT-PCR analyses from total RNA samples (means $\pm \mathrm{SD}, \mathrm{n}=3,{ }^{*} \mathrm{p}<0,01$ ). $\boldsymbol{C}$, The $\mathrm{c}$-jun protein was detected inside $\mathrm{T}_{-\mathrm{MV}}$ 作 Western Blotting. Whole cell lysates $(=\mathrm{C})$ were used as positive control. $\boldsymbol{D}$, However, inhibition of c-jun signaling with a JNK-inhibitor (JNK-I) was not able to antagonize MV-induced tumor invasion of MCF-7 cells (microinvasion assays, means $\pm \mathrm{SD}, \mathrm{n}=3$ ). Modified from (Menck et al, 2014b).

\subsection{EV as mediators of tumor-macrophage interactions}

The local microenvironment is increasingly recognized as important factor in the establishment of a favorable tumor niche. Especially tumor-infiltrating $\mathrm{M} \phi$ are known for their protumoral effects (Allavena et al, 2008). Our group has already shown that co-culture of the breast cancer cell lines MCF-7 and SK-BR-3 with human M $\phi$ increases tumor cell invasion (Hagemann et al, 2004). The tumor-promoting effect is dependent on an induction of Wnt5a in the latter (Pukrop et al, 2006). However, so far it could not be elucidated how this pro-invasive tumor-stroma crosstalk is mediated. For this reason, we aimed to investigate if $\mathrm{EV}$ are the missing link and mediate intercellular communication. 


\subsubsection{T-MV do not induce a typical M2 phenotype in human $M \phi$}

We first analyzed which effects T-MV exert on human $\mathrm{M} \phi$. For these studies, monocytes were isolated from Buffy coats and further differentiated to $\mathrm{M} \phi$ in the presence of low concentrations of macrophage colony-stimulating factor (M-CSF) which is described in detail in (Menck et al, 2014a). Since the differentiation method influences the phenotype of the obtained $\mathrm{M} \phi$, they were first characterized by flow cytometry (Fig. 19). Immunophenotyping confirmed that the cells were positive for typical markers for mature $M \phi$, i.e. CD45, CD14, $\mathrm{CD} 11 \mathrm{c}$ and CD11b as well as the mannose receptor CD206 corroborating that these cells represent a population of mature $\mathrm{M} \phi$.
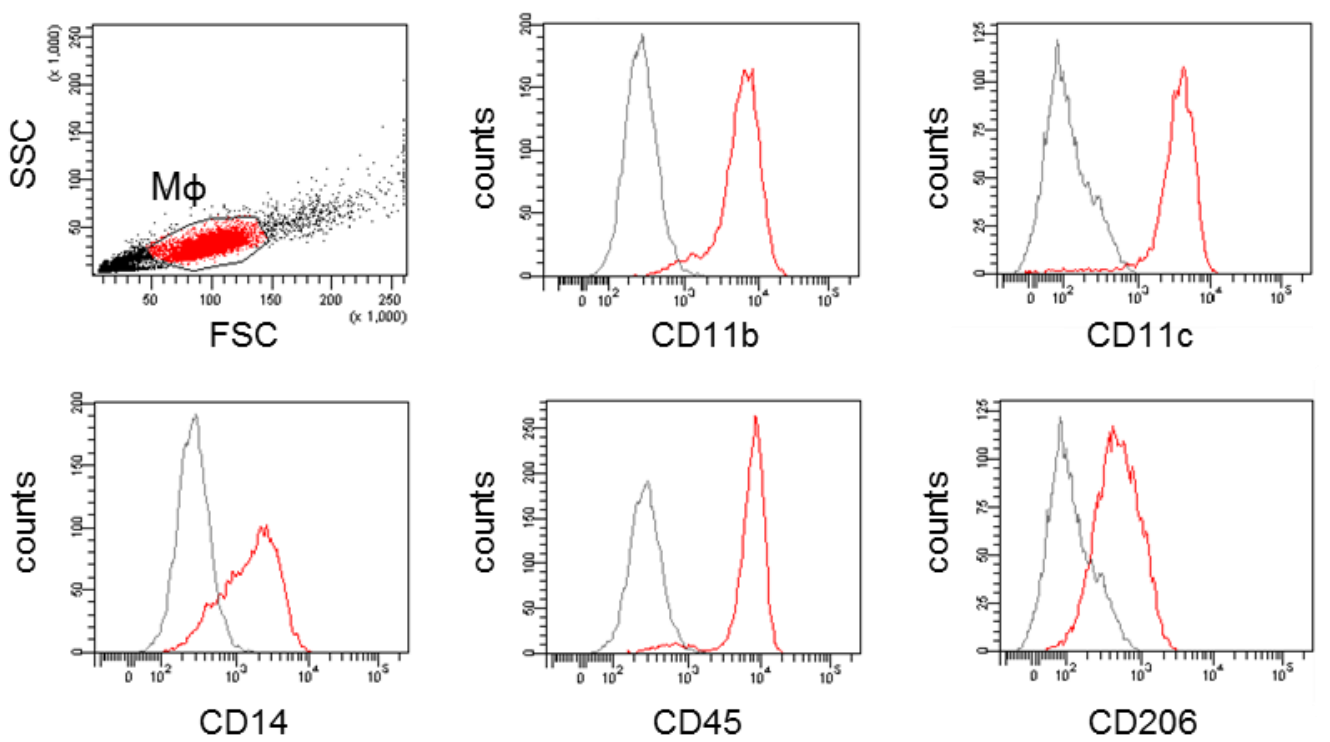

Fig. 19: Immunophenotype of isolated human $M \phi$

Monocytes were isolated from Buffy Coats and differentiated to $\mathrm{M} \phi$ in the presence of low concentrations of $\mathrm{M}$ CSF. M $\phi$ were characterized for the expression of typical markers by flow cytometry (indicated in red). The corresponding isotype controls are shown in grey. Modified from (Menck et al, 2014a).

Tumor-infiltrating $\mathrm{M} \phi$ are often described to acquire an anti-inflammatory M2 phenotype due to tumor- and immune cell-derived cytokines present in the tumor stroma (Mantovani et al, 2002). For this reason, we investigated if stimulation with T-MV contributes to the induction of a tumor-supporting phenotype in $\mathrm{M} \phi$. However, stimulation with $\mathrm{T}-\mathrm{MV}_{\mathrm{M}}$ did not influence the expression or activation of selected pro-inflammatory M1 (i.e. IL-1ß, TNF $\alpha, \mathrm{IL}-12$ ) or anti-inflammatory M2 (i.e. IL-10) cytokines (Fig. 20A+B). Only P-MV which were used as negative control slightly induced $I L-1 \beta$ mRNA expression which might point towards a shift to a pro-inflammatory $\mathrm{M} \phi$ phenotype. However, there was no significant increase in secreted IL-1ß arguing against such effect. Moreover, also the expression of other protumoral factors including the vascular endothelial growth factor (VEGFA), which promotes angiogenesis, or 
selected matrix metalloproteinases $(M M P)$, which mediate extracellular matrix degradation, remained unchanged in M $\phi$ upon MV stimulation (Fig. 20A, C).
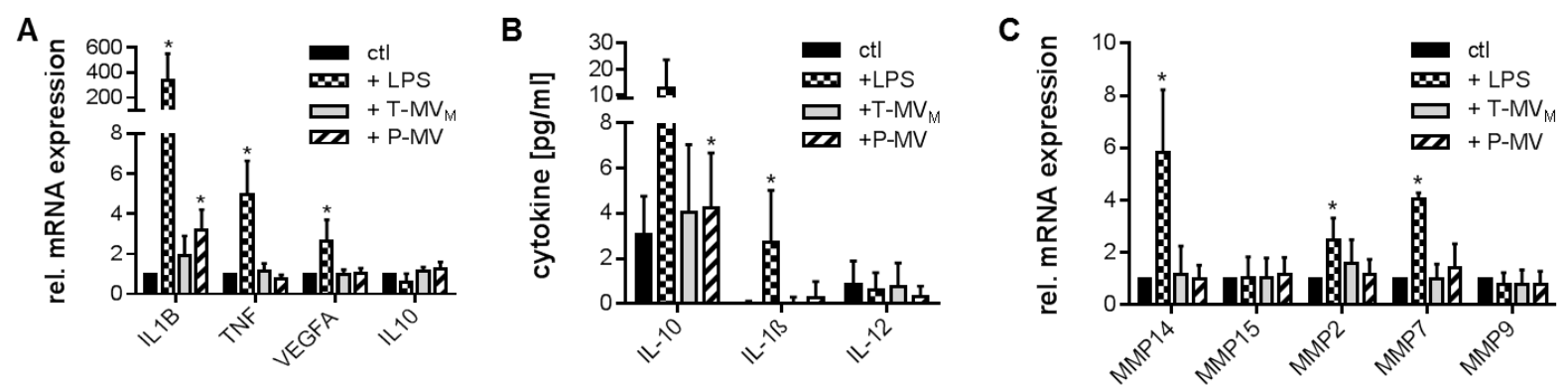

Fig. 20: Analysis of the $M \phi$ phenotype after MV stimulation

Human M $\phi$ were stimulated for $24 \mathrm{~h}$ with $25 \mu \mathrm{g} / \mathrm{ml} \mathrm{T-MV}$ M or P-MV (negative control). LPS (100 ng/ml) was used as positive control. $\boldsymbol{A}+\boldsymbol{B}$, Expression of typical pro- or anti-inflammatory cytokines was analyzed by qRTPCR $(A)$ or ELISA $(B)\left(\right.$ mean $\left.\pm \mathrm{SD}, \mathrm{n}=5,{ }^{*} \mathrm{p}<0,05\right)$. $\boldsymbol{C}$, Relative mRNA expression of selected MMP in M $\phi$ (qRT$\mathrm{PCR}$, mean $\pm \mathrm{SD}, \mathrm{n}=5,{ }^{*} \mathrm{p}<0,05$ ). Figure taken from (Menck et al, 2013).

\subsubsection{T-MV are responsible for the induction of Wnt5a in human $M \phi$}

We next wanted to know whether T-MV might be responsible for the observed WNT5A induction in $\mathrm{M} \phi$ under co-culture conditions. While the role of Wnt5a in cancer progression is controversially discussed, there is increasing evidence that its expression induces an immunosuppressive, tumor-supporting phenotype in human $\mathrm{M} \phi$ (Bergenfelz et al, 2012; Pukrop et al, 2006).
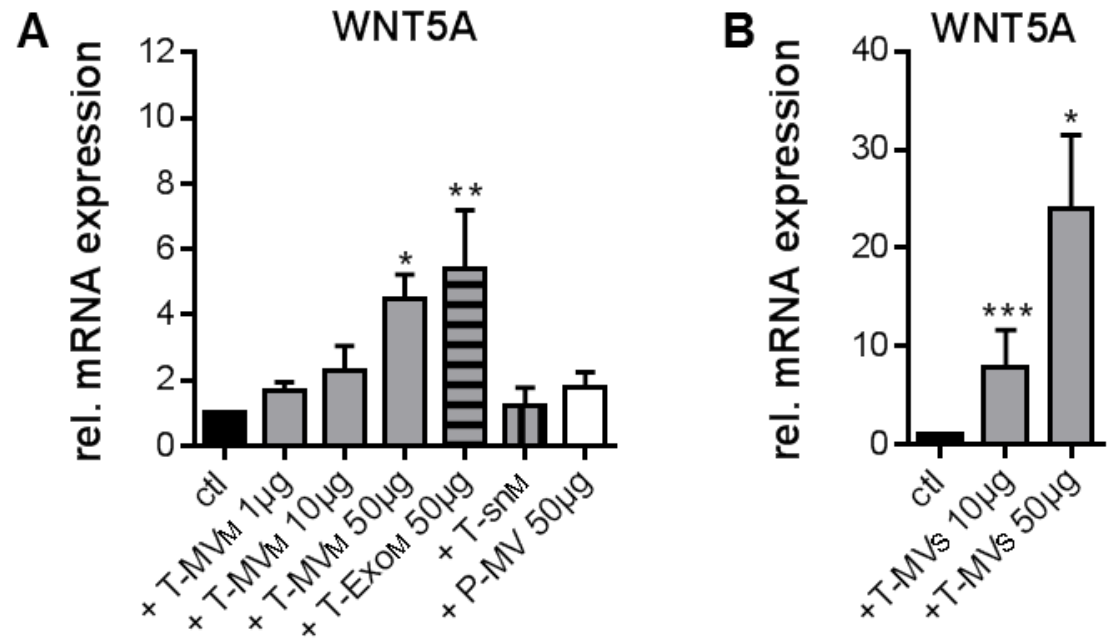

Fig. 21: T-EV induce Wnt5a in human $M \phi$

$\boldsymbol{A}+\boldsymbol{B}$, Human $\mathrm{M} \phi$ were stimulated for $24 \mathrm{~h}$ with increasing concentrations of $\mathrm{T}-\mathrm{MV}_{\mathrm{M}}, \mathrm{T}-\mathrm{Exo}_{\mathrm{M}}(\mathrm{A})$ or $\mathrm{T}-\mathrm{MV} \mathrm{S}(\mathrm{B})$ and qRT-PCR was carried out from total RNA samples. Benign MV from human platelets $(\mathrm{P}-\mathrm{MV})$ and the particle-free supernatant $\left(\mathrm{T}-\mathrm{sn}_{\mathrm{M}}\right)$ were used as negative controls (means $\pm \mathrm{SEM}, \mathrm{n} \geq 5, * \mathrm{p}<0,001, * * \mathrm{p}<0,01$, $* * * \mathrm{p}<0,05)$. Modified from (Menck et al, 2013). 
Indeed, stimulation with T-MV led to a concentration-dependent upregulation of WNT5A in human $\mathrm{M} \phi$ (Fig. 21). The same effect was also observed for stimulation with T-Exo. However, neither benign P-MV nor the vesicle-free tumor cell supernatant (T-sn) induced WNT5A indicating that this is a specific feature of tumor cell-derived EV.

Since MV have often been described as carriers of nucleic acids, we analyzed whether the increase in WNT5A mRNA was due to horizontal transfer. T-MV samples were used for mRNA isolation and were subjected to qRT-PCR. Agarose gel electrophoresis was used to visualize the amplified gene-specific product and confirmed the presence of WNT5A mRNA inside the vesicles (Fig. 22A). Nonetheless, inhibition of $\mathrm{M} \phi$ gene transcription by the chemotherapeutic drug Actinomycin D, in concentrations which did not affect cell viability (Fig. 22B), antagonized the observed WNT5A induction by T-MV (Fig. 22C). This finding indicates that WNT5A is newly transcribed after MV stimulation.

\section{A $\quad$ MDA $\quad$ MCF-7 T-MV $\mathrm{M}_{\mathrm{M}} \quad \mathrm{NTC}$}
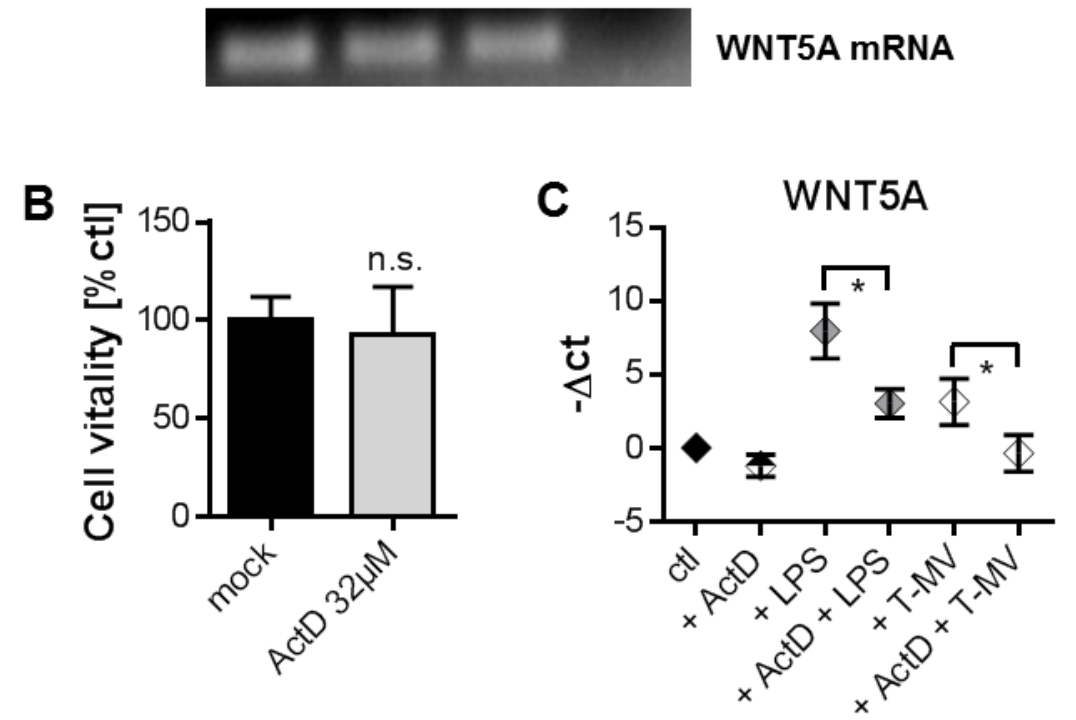

Fig. 22: Wnt5a is newly transcribed in $M \phi$ after T-MV stimulation

$\boldsymbol{A}$, Agarose gel: Wnt5a mRNA was visualized in T-MV after PCR amplification. MDA-MB231 (MDA) and MCF-7 are displayed as positive controls. NTC $=$ No-Template-Control. $\boldsymbol{B}$, ActD $(32 \mu \mathrm{M})$ has no effect $\mathrm{M} \phi$ viability as assessed by MTT assay (means $\pm \mathrm{SD}, \mathrm{n}=3$, n.s. $=$ not significant). $\boldsymbol{C}$, qRT-PCR: Pre-incubation of $\mathrm{M} \phi$ with ActD significantly reduces Wnt5a induction by LPS (100 ng/ml, positive control) and T-MV $\mathrm{M}_{\mathrm{M}}(25 \mu \mathrm{g} / \mathrm{ml})$ after $24 \mathrm{~h}$ of stimulation (means $\pm \mathrm{SD}, \mathrm{n}=3,{ }^{*} \mathrm{p}<0,05$ ). Modified from (Menck et al, 2013).

In order to analyze whether uptake of T-MV is required for the observed WNT5A induction, $\mathrm{M} \phi$ were incubated with $\mathrm{T}-\mathrm{MV}_{\mathrm{M}}$ which had been previously labeled with the red membraneintercalating dye PKH26. After $24 \mathrm{~h}$ an uptake of T-MV into $\mathrm{M} \phi$ was clearly visible by fluorescence microscopy (Fig. 23A). Additionally, we performed confocal microscopy to confirm that the vesicles are indeed incorporated into the cells and not only stick to the 
cellular surface (Fig. 23B). The images showed that T-MV were found as vesicle-like structures in the cytoplasm of the cells, whereas the plasma membrane of the cells remained unstained. For analysis of MV uptake kinetics, we stimulated M $\phi$ with PKH26-labeled T$\mathrm{MV}_{\mathrm{M}}$ and measured the red fluorescence in the supernatant as well as in the cells after different time points. Already $1 \mathrm{~h}$ after stimulation a significant decrease of the fluorescence in the supernatant was observed, while in parallel there was a constant increase of cellular fluorescence (Fig. 23C) which further confirms a time-dependent uptake of T-MV into human $\mathrm{M} \phi$. The strongest fluorescence in the cells was observed after $24 \mathrm{~h}$.

A

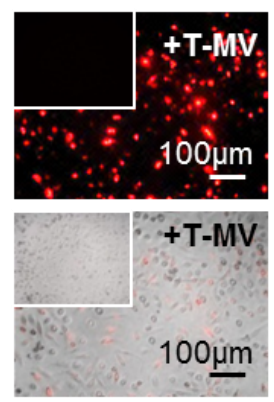

B

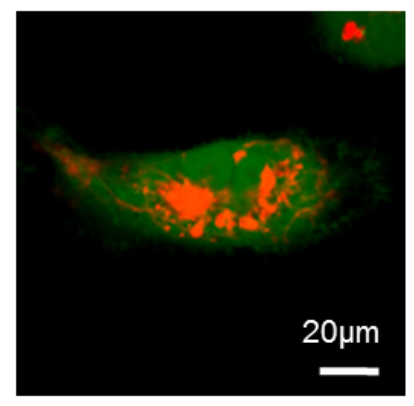

C

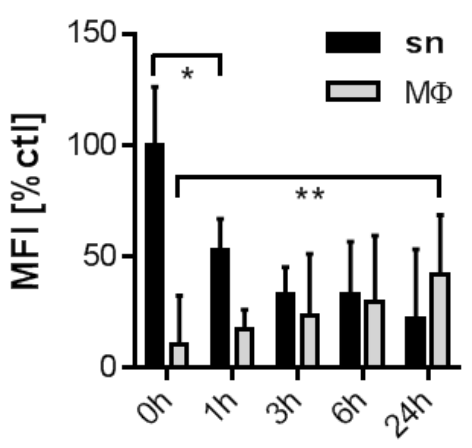

D

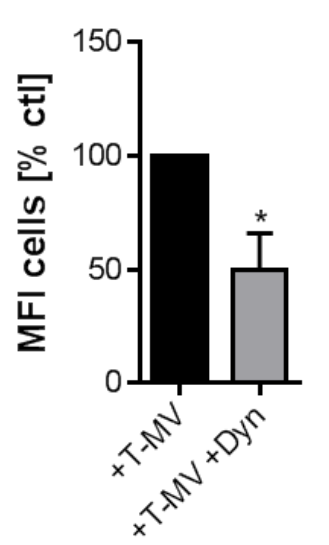

$\mathbf{E}$

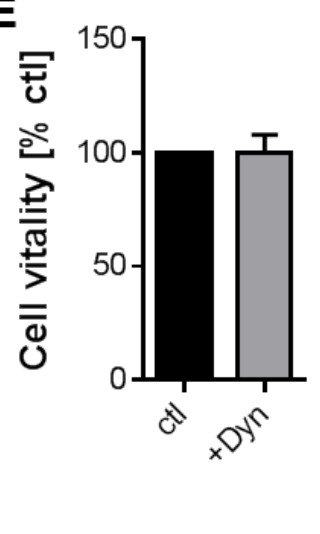

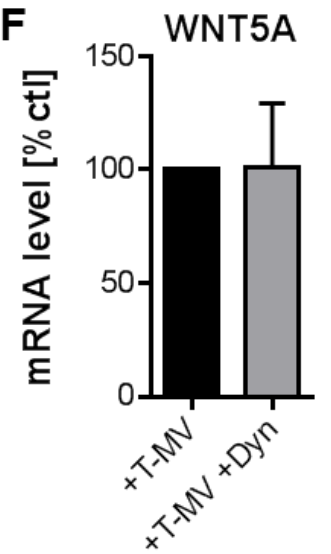

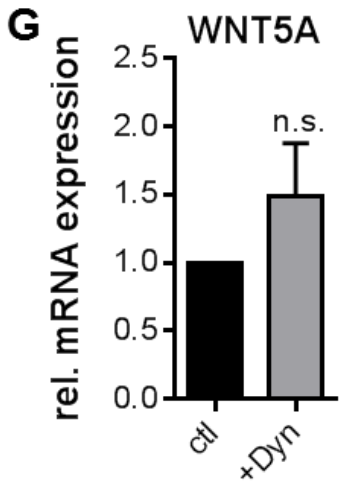

Fig. 23: Human $M \phi$ incorporate T-MV, but their uptake is not essential for Wnt5a induction

Stimulation of human M $\phi$ with PKH26-labeled T-MV $\mathrm{M}_{\mathrm{M}} \boldsymbol{A} \boldsymbol{A} \boldsymbol{B}$, Uptake was visualized after $24 \mathrm{~h}$ by fluorescence microscopy (A) (magnification: 10x, inserts: unstimulated control) or confocal microscopy $(B)$ (magnification: 40x, green: calcein). $\boldsymbol{C}$, The distribution of the red fluorescence in supernatant and cells was measured by flow cytometry (means $\pm \mathrm{SD}, \mathrm{n}=5, * \mathrm{p}<0,01, * * \mathrm{p}<0,05$ ). $\boldsymbol{D}$, MV uptake into $\mathrm{M} \phi$ can be significantly reduced by treatment with $12,5 \mu \mathrm{M}$ dynasore (means $\pm \mathrm{SD}, \mathrm{n}=3,{ }^{*} \mathrm{p}<0,05$ ). $\boldsymbol{E}$, Cell viability is not influenced by dynasore (MTT-assay, 24h, mean $\pm \mathrm{SD}, \mathrm{n}=3$ ). $\boldsymbol{F}+\boldsymbol{G}$, Dynasore neither influences $\mathrm{M} \phi-\mathrm{Wnt5a}$ expression itself, nor does it inhibit T-MV-meditaed Wnt5a expression (qRT-PCR, mean \pm SD, $n \geq 9$, n.s. = not significant). Modified from (Menck et al, 2013).

Next, we pre-incubated $\mathrm{M} \phi$ with dynasore which was able to reduce MV uptake into $\mathrm{M} \phi$ by around $50 \%$ without affecting cellular viability (Fig. 23D+E). In spite of inhibiting cellular MV uptake, WNT5A induction by $\mathrm{T}-\mathrm{MV}_{\mathrm{M}}$ was not reduced, while also dynasore itself did not influence WNT5A mRNA levels (Fig. 23F+G). 


\subsubsection{M $\phi$ export the produced Wnt5a protein on own responding EV}

We further analyzed whether the observed induction of WNT5A expression was also translated to the protein level. Indeed, after $24 \mathrm{~h}$ of stimulation the Wnt5a protein was increasingly detectable inside the $\mathrm{M} \phi$ (Fig. 24A). Next, we aimed to investigate whether and how the produced Wnt5a protein is exported from the $\mathrm{M} \phi$. Since Wnt proteins are very hydrophobic, their mobility in the extracellular space is limited. The question of how Wnt proteins travel through tissues is still under debate. First hints that Wnt proteins might be associated with membrane vesicles were presented in a study from Greco et al who showed a colocalization of the Drosophila Wnt1 homolog, Wingless, with exosomes-like vesicles, the so-called argosomes (Greco et al, 2001). In order to analyze whether the produced Wnt5a protein can be transported on MV and Exo in the human system, we investigated Wnt5a expression on EV which are secreted by Wnt5a-positive M $\phi$.

However, since a large amount of $\mathrm{M} \phi$ is needed to isolate sufficient numbers of $\mathrm{EV}$ for further analysis which, in turn, would require a high quantity of T-MV for stimulation, we developed an alternative protocol for $\mathrm{M} \phi$ activation: $\mathrm{M} \phi$ were seeded in 6-well-plates on ECM gel ( $80 \mu \mathrm{l} /$ well, diluted 1:16 in culture medium) and stimulated for $24 \mathrm{~h}$ with tumor cell supernatant which still contains all MV, Exo and soluble factors (for further details see 2.2.1.6). This procedure led to a significant induction of WNT5A (Fig. 24B). Moreover, it prevented the inefficient and time-consuming isolation of pure vesicle populations by differential ultracentrifugation. Since after $24 \mathrm{~h}$ most of the T-MV in the tumor cell supernatant are ingested by $\mathrm{M} \phi$ (Fig. 23C) and MCF-7 cells are negative for Wnt5a (Fig. 27C), no contaminating Wnt5a signals are to be expected from T-MV.

The generated Wnt5a-positive M $\phi$ were then used for EV isolation. Surprisingly, Wnt5a was detected on M $\phi$-MV by electron microscopy in a Wnt5a-immunogold staining where it clearly localized to the membrane of the vesicles (Fig. 24D). To confirm this finding, we further analyzed M $\phi-E V$ by Western Blotting and indeed found Wnt5a on both vesicle populations, M $\phi$-MV as well as M $\phi$-Exo (Fig. 24C). We then investigated whether the Wnt5a-carrying M $\phi$-EV had any influence on tumor invasion. As shown in Fig. 24E, MV and Exo from stimulated M $\phi$ significantly enhanced MCF-7 cell invasion, while neither the particle-free $\mathrm{M} \phi$ supernatant nor benign P-MV had any influence on tumor invasion. 
A

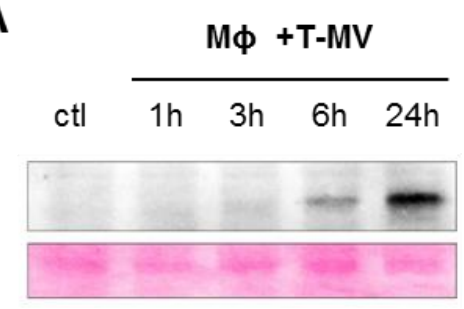

B

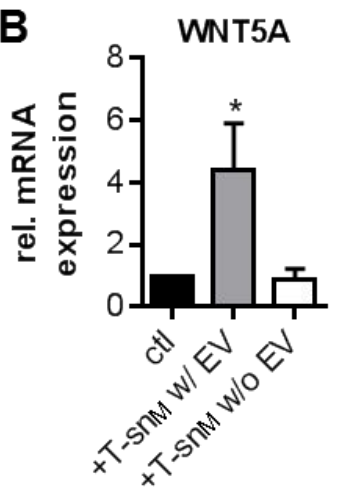

C

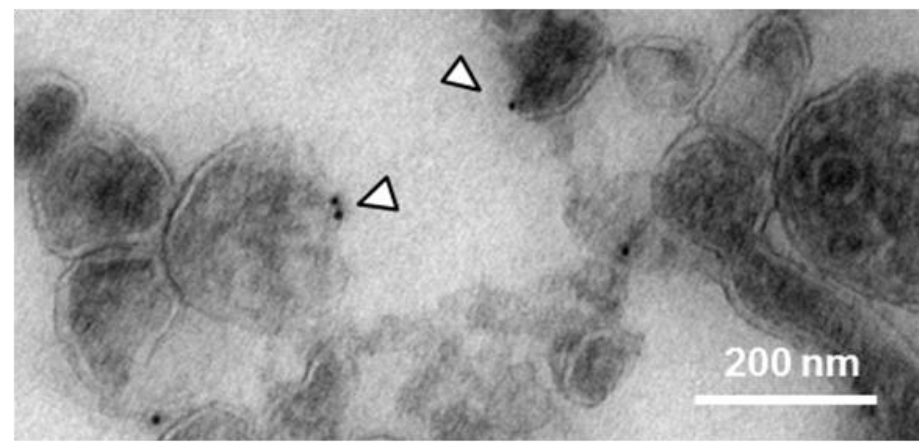

D

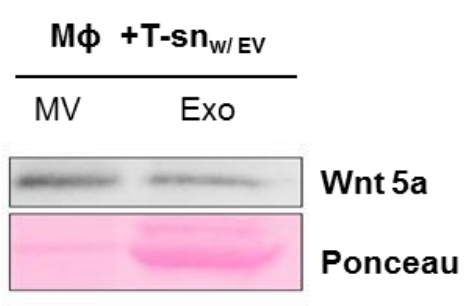

Wnt 5a (42kDa)

Ponceau 
expression. Subsequently, we layered the MV and Exo of the Wnt5a-positive cell line SKBR-3 separately on top of two sucrose gradients and analyzed the distribution of the vesicles based on the previously described markers (Fig. 25B). Wnt5a clearly co-localized with the MV marker EMMPRIN in sucrose gradients from $T-M_{S}$ as well as with the exosome marker Tsg101 in gradients from $\mathrm{T}^{-E \mathrm{E}_{\mathrm{S}}}$ confirming the presence of Wnt5a on both vesicle populations.

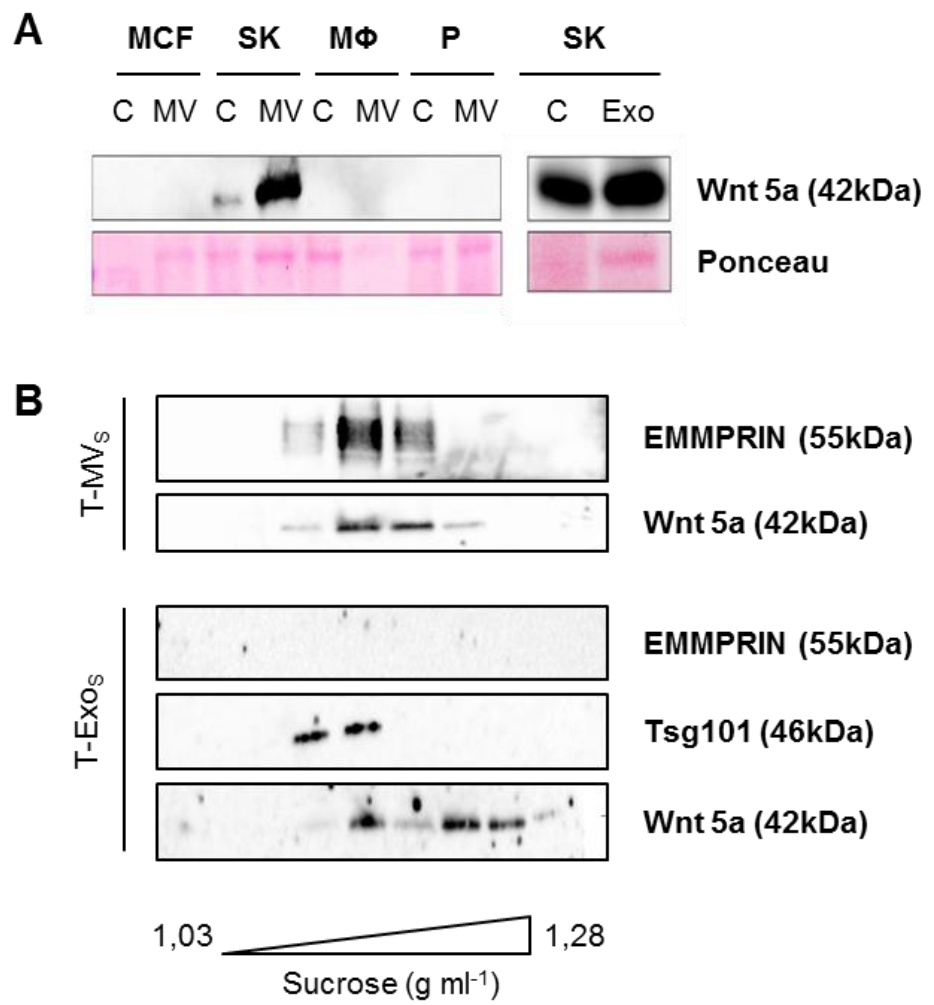

Fig. 25: Wnt5a is expressed on MV and Exo

$A$, Western Blot: Comparative analysis of the Wnt5a expression on MV (and Exo) from MCF-7 (MCF) and SKBR-3 (SK) breast cancer cells as well as on unstimulated $\mathrm{M} \phi$ or platelets $(\mathrm{P}) . \boldsymbol{B}$, T-MV $\mathrm{M}_{\mathrm{S}}$ and $\mathrm{T}-\mathrm{Exo}_{\mathrm{S}}$ were layered separately on sucrose gradients and the distribution of Wnt5a and known MV/Exo markers was analyzed by Western Blotting. Taken from (Menck et al, 2013).

\subsubsection{MV-induced Wnt5a expression is mediated through activation of p38/MAPK which can be antagonized by the Wnt-inhibitor DKK-1}

After having shown that T-MV are responsible for the Wnt5a expression in human M $\phi$, we tried to elucidate which upstream mediators are activated in $\mathrm{M} \phi$ after T-MV stimulation and mediate WNT5A induction. Therefore, we stimulated $\mathrm{M} \phi$ with $\mathrm{T}-\mathrm{MV}_{\mathrm{M}}$ and analyzed the activation of several signaling pathways after different time points. While levels of PKC, CamKII or c-jun remained unchanged (data not shown) indicating that the non-canonical $\mathrm{Wnt} / \mathrm{Ca}^{2+}$ or PCP signaling pathway are not affected, we observed an activation of the p38 (MAPK14) signaling pathway already $1 \mathrm{~h}$ after stimulation with T-MV (Fig. 26A). Pre- 
incubation of $\mathrm{M} \phi$ with the p38-inhibitor SB-203580 (see 3.1.8) significantly decreased WNT5A induction mediated by T-MV without affecting M $\phi$ viability (Fig. 26B+C).

Furthermore, it has already been shown that the pro-invasive effect of $\mathrm{M} \phi$ on tumor invasion can be blocked by the Wnt-inhibitor DKK-1 (Pukrop et al, 2006). However, so far we could not explain how and on which of the cells involved, M $\phi$ or tumor cells, DKK-1 exerts this effect. Since our results suggest MV and Exo as main mediators of tumor-M $\phi$ crosstalk, we were now able to dissect the various aspects of intercellular communication, namely T-MVinduced Wnt5a upregulation and $\mathrm{M} \phi-\mathrm{MV}$ induced tumor invasion, under co-culture conditions. To this end, we performed microinvasion assays in which we co-cultured MCF-7 breast cancer cells either with whole $\mathrm{M} \phi$ or with isolated $\mathrm{M} \phi-\mathrm{MV}$ from activated $\mathrm{M} \phi$ and then analyzed the influence of DKK-1 on their pro-invasive effects (Fig. 26D).

A

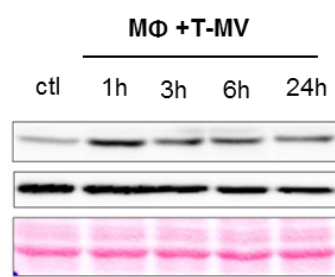

P-p38 (41kDa) p38 (38kDa)

Ponceau
B
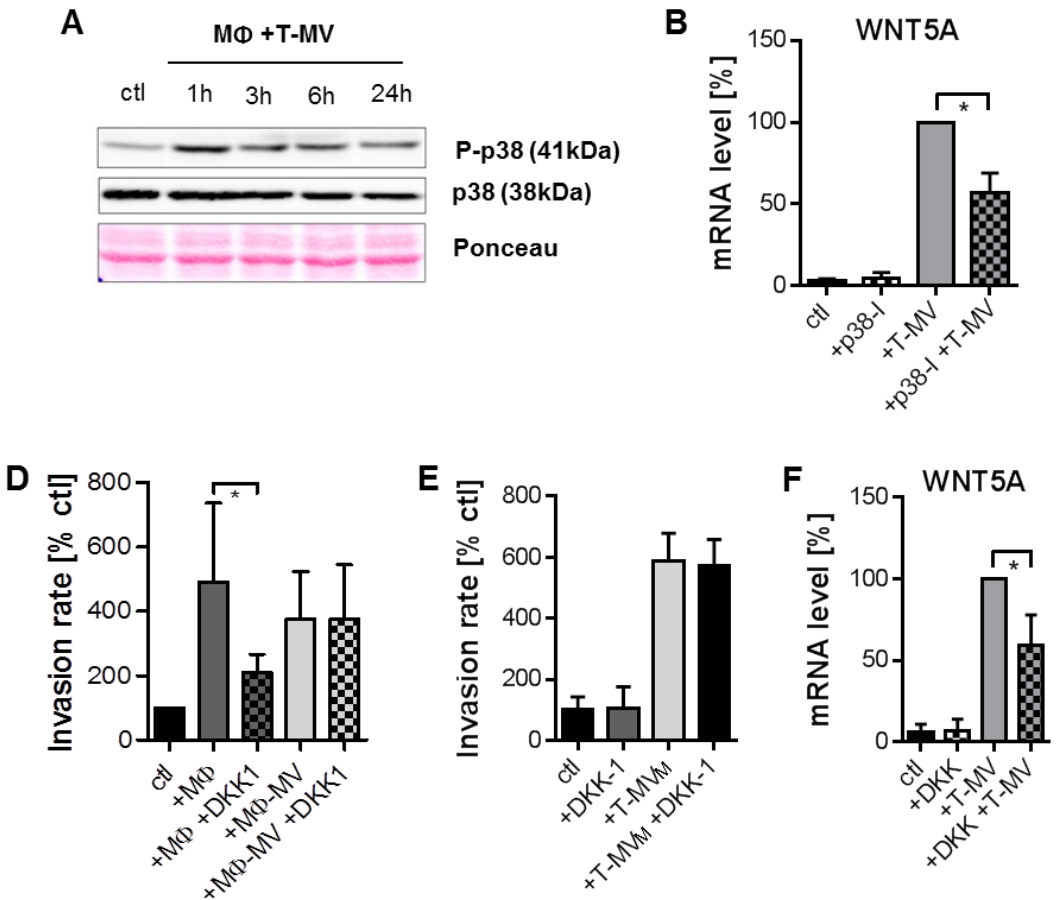
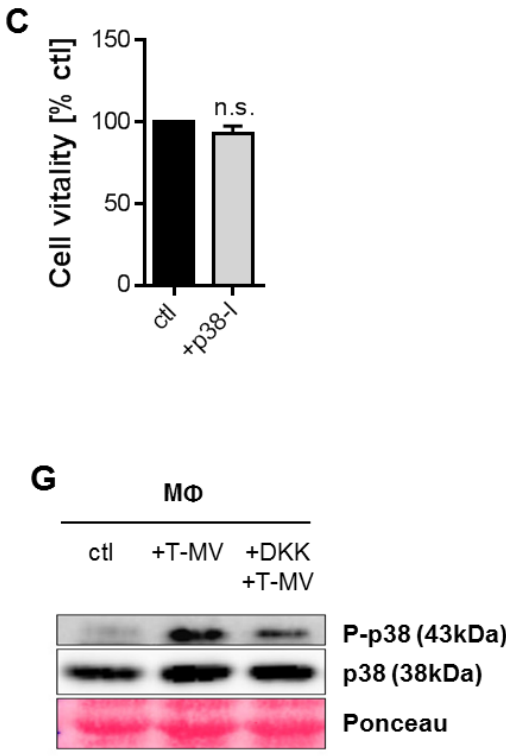

Fig. 26: MV-mediated Wnt5a induction in M $\phi$ is mediated by p38/MAPK signaling which can be antagonized by DKK1

$A$, $\mathrm{M} \phi$ were stimulated with $\mathrm{T}-\mathrm{MV}_{\mathrm{M}}(25 \mu \mathrm{g} / \mathrm{ml})$ and activation of the $\mathrm{p} 38$ signaling pathway at the indicated time points was analyzed by Western Blotting. $\boldsymbol{B}$, Pre-incubation ( $2 \mathrm{~h}$ ) of $\mathrm{M} \phi$ with the p38 inhibitor SB-203580 (p38I, $0,5 \mu \mathrm{M})$ significantly reduces T-MV-mediated WNT5A induction. $C, \mathrm{M} \phi$ viability during $24 \mathrm{~h}$ of treatment with $0,5 \mu$ M SB-203580 (p38-I) was assessed by MTT assay. $\boldsymbol{D}$, Microinvasion assay of MCF-7 cells with or without pre-incubation with DKK-1 (200ng/ml) followed by co-culture of the cells with M $\phi$ or MV derived from activated M $\phi . \boldsymbol{E}+\boldsymbol{F}$, Pre-incubation of M $\phi$ with DKK-1 (200 ng/ml) significantly reduces WNT5A induction by T-MV in qRT-PCR $(E)$ or phosphorylation of p38 in Western Blots $(F)$. Modified from (Menck et al, 2013).

Interestingly, DKK-1 was able to antagonize the pro-invasive effect of the whole $\mathrm{M} \phi$, but did not influence the pro-invasive potential of M $\phi-M V$. In parallel, DKK-1 did not decrease the pro-invasive autologous effect of $\mathrm{T}-\mathrm{MV}_{\mathrm{M}}$ on tumor invasion (Fig. 26E). This led us to the 
hypothesis that DKK-1 does not affect the tumor cells, but interferes with the induction of Wnt5a in $M \phi$ and thus their acquisition of a tumor-promoting phenotype. Indeed, preincubation of $\mathrm{M} \phi$ with DKK-1 significantly antagonized MV-mediated WNT5A induction (Fig. 26F). In line with this, DKK-1 further diminished p38 activation after T-MV stimulation confirming a link between both (Fig. 26G).

\subsubsection{MV-bound Wnt5a is taken up by the tumor cells}

Since T-MV stimulation induced the expression of the Wnt5a protein which was exported by activated $\mathrm{M} \phi$ on their EV, we analyzed if vesicle-bound Wnt5a can then be transferred to and taken up by the tumor cells which would complete the pro-invasive communication loop between tumor cells and $\mathrm{M} \phi$. First of all, we investigated if $\mathrm{M} \phi-\mathrm{MV}$ can be ingested by breast cancer cells. For this reason MCF-7 cells were stimulated with PKH26-labeled M $\phi$-MV followed by fluorescence microscopy. Indeed, after $24 \mathrm{~h}$ the red M $\phi-\mathrm{MV}$ were detected inside the cancer cells (Fig. 27A).

A

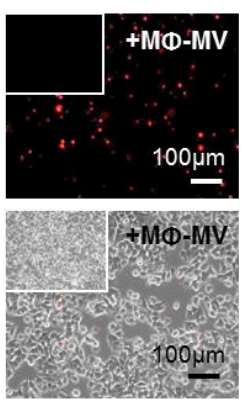

B

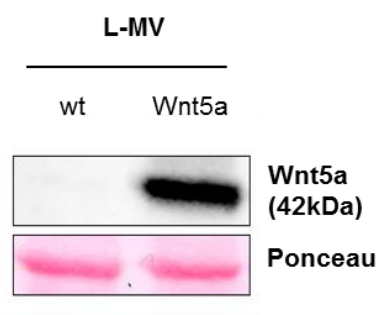

C

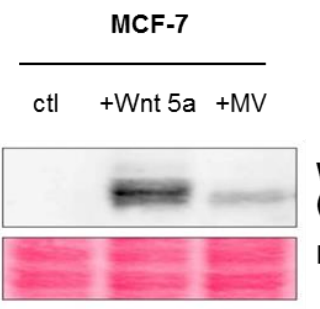

Wnt5a

(42kDa)

Ponceau

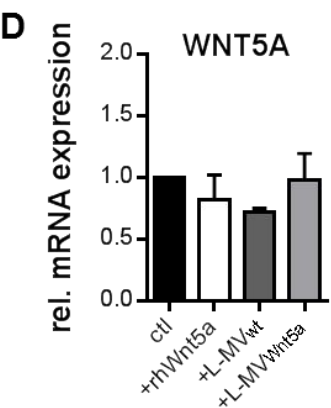

Fig. 27: Wnt5a-positive stroma-derived MV are ingested by breast cancer cells

$\boldsymbol{A}$, MCF-7 were stimulated for $24 \mathrm{~h}$ with PKH26-labeled MV from activated M $\phi$. Uptake was visualized by fluorescence microscopy (magnification: 10x, inserts: unstimulated control). $\boldsymbol{B}$, Western Blot: Wnt5a is present at high levels on MV from Wnt5a-overexpressing (Wnt5a) compared to wild type L cells (wt). $\boldsymbol{C}+\boldsymbol{D}$, Stimulation of Wnt5a-negative MCF-7 cells with rhWnt5a or L-MV $\mathrm{Wnt5a}_{\mathrm{a}}$ leads to the detection of the Wnt5a protein inside the cells $(C$, Western Blot) while there is no induction of WNT5A on the mRNA level in MCF-7 (D). Modified from (Menck et al, 2013).

For further analysis of Wnt5a uptake, we decided to work with Wnt5a-overexpressing L cells (Wnt5a-L cells) as model cell line for Wnt5a-positive $\mathrm{M} \phi$ since the generation of activated $\mathrm{M} \phi$ still is a comparably expensive and time-consuming method. Wnt5a-L cells are a murine fibroblast cell line and can therefore be considered as an additional variant of tumor stroma cells. As these cells overexpress Wnt5a, it can also be found enriched on their released MV $\left(\mathrm{L}-\mathrm{MV}_{\mathrm{Wnt5a}}\right.$ ) (Fig. 27B). Stimulation of the Wnt5a-negative MCF-7 cells with Wnt5a-positive $\mathrm{L}-\mathrm{MV}_{\mathrm{Wnt5}}$ led to the detection of the Wnt5a protein inside the tumor cells (Fig. 27C). High 
doses of recombinant Wnt5a (rhWnt5a) were used as respective positive control and were also taken up by MCF-7. To exclude an induction of WNT5A which could also be responsible for the observed effect, we analyzed its expression in MCF-7 stimulated with L-MV $\mathrm{Wnt5a}_{\text {(Fig. }}$ 27D). However, WNT5A mRNA levels remained unchanged, arguing for an uptake of MVbound Wnt5a.

\subsubsection{The role of Evi for Wnt5a expression on MV and Exo}

After having identified the release and transfer of Wnt5a from activated $\mathrm{M} \phi$ to the tumor cells, we asked how Wnt5a is transported on EV. Transmission electron microscopy had revealed an association of Wnt5a with the membrane of the vesicles (see Fig. 24D). However, since Wnt proteins in general are palmitoylated by the acyltransferase porcupine in the endoplasmic reticulum (Tanaka et al, 2000; Willert et al, 2003), they are highly hydrophobic which would allow their unspecific association with membranes through hydrophobic interactions. Indeed, incubation of the Wnt-negative $\mathrm{T}^{-\mathrm{MV}_{\mathrm{M}}}$ and $\mathrm{T}-\mathrm{Exo}_{\mathrm{M}}$ with high doses of rhWnt5a (see 2.2.2.4) led to unspecific binding of the protein to the vesicles (Fig. 28A). However, a significant part of the protein still remained in the EV-free supernatant after ultracentrifugation.

Nonetheless, a specific association of canonical Wnt3a with the protein Evi has recently been described which is essential for Wnt secretion on Exo (Gross et al, 2012). Based on this finding we asked whether Evi is also required for Wnt expression on MV. Hence, we performed a stable knockdown of Evi in the Wnt5a-positive cell line SK-BR-3 as well as in Wnt5a-L cells (see 2.2.1.2). Expression of Evi/WLS was reduced by around $75 \%$ in Evi knockdown (shEvi) compared to non-sense control (ns ctl) cells on the mRNA level and was no longer detectable on the protein level (Fig. 28B+C). Although several commercially available antibodies against murine Evi had been tested, none gave reproducible results. For this reason, Evi expression and knock-down efficiency could only be confirmed on the protein level for human SK-BR-3, but not for murine Wnt5a-L cells. Evi expression was also lost on MV and Exo derived from SK-BR-3 shEvi cells (Fig. 28D).

Evi knockdown resulted in a loss of Wnt5a expression on $\mathrm{T}-\mathrm{MV}_{\mathrm{S}} / \mathrm{T}-\mathrm{Exo}_{\mathrm{S}}$ as well as L$\mathrm{MV}_{\text {Wnt5a }} / \mathrm{L}-\mathrm{ExO}_{\mathrm{Wnt5a}}$ (Fig. 28E) while WNT5A expression in shEvi cells remained unchanged in SK-BR-3 and was only slightly diminished in Wnt5a-L cells (Fig. 28F). The results suggest that the export of Wnt5a on EV is impaired in shEvi cells which should lead to an 
augmentation of the protein inside the cells as it was described for Wnt3a in Wnt3aoverexpressing shEvi L cells (Gross et al, 2012).

A

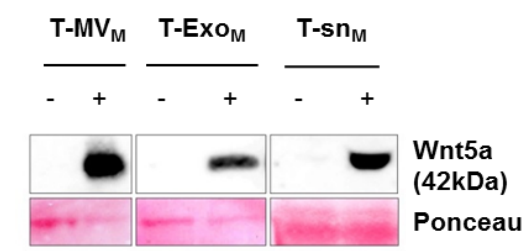

B

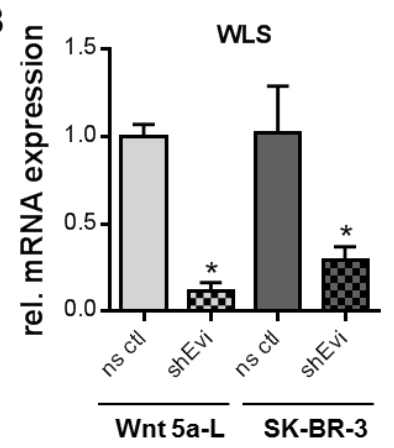

C
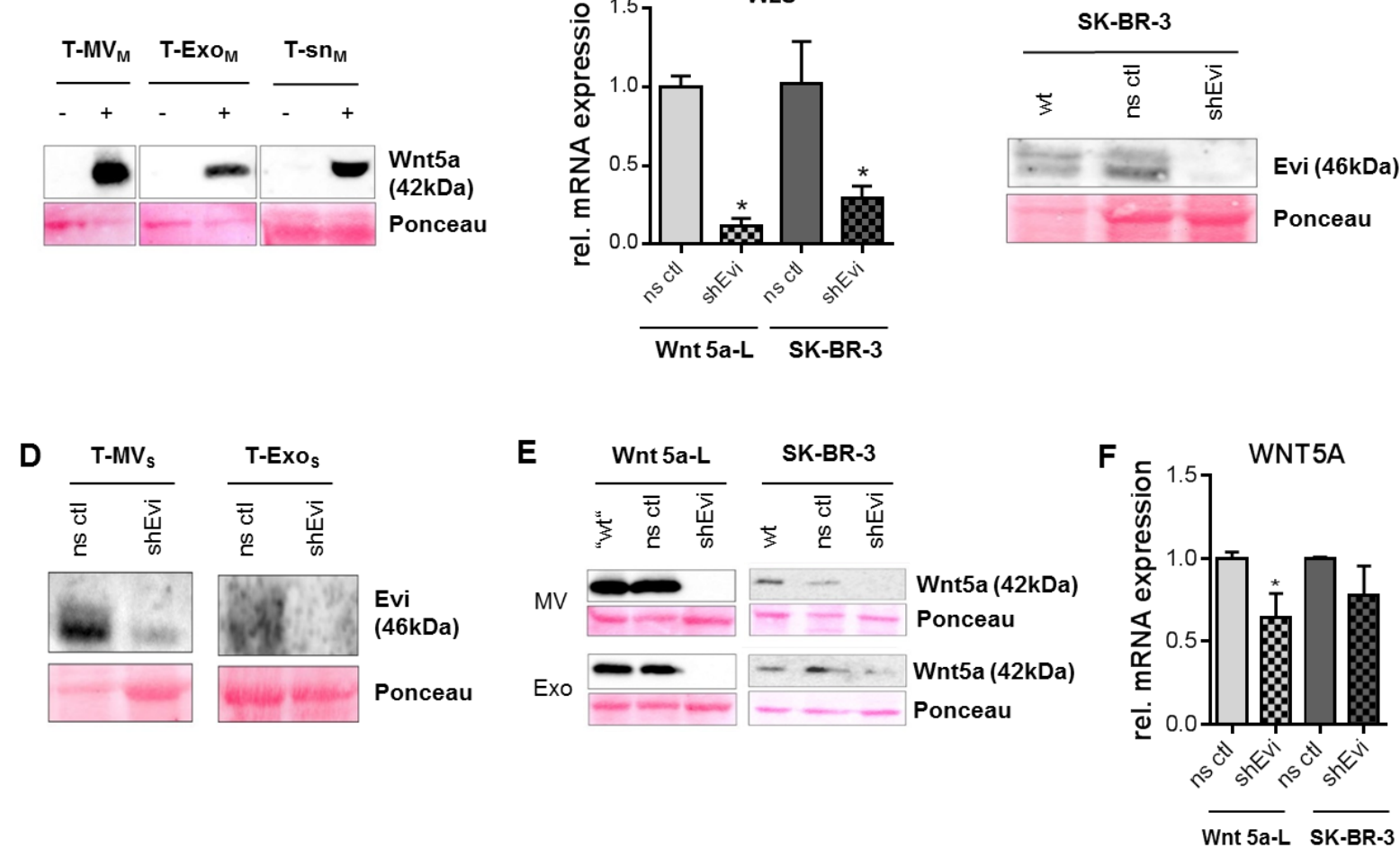

Fig. 28: Wnt5a is associated with $\mathrm{EV}$ in a nonspecific as well as specific, Evi-dependent way

$\boldsymbol{A}$, Wnt-negative $\mathrm{T}-\mathrm{MV}_{\mathrm{M}}$ and $\mathrm{T}-\mathrm{Exo}_{\mathrm{M}}$ were incubated with (+) or without (-) $100 \mathrm{ng} / \mathrm{ml} \mathrm{rhWnt5a}$ which led to unspecific binding of the protein to the vesicles, while significant amounts of the protein still remained in the supernatant (Western Blot). B, Expression of Evi/WLS was analyzed in SK-BR-3 and Wnt5a-L Evi knockdown (shEvi) and non-sense control (ns ctl) cells by qRT-PCR (means \pm SD, n=3, *p<0,05). $\boldsymbol{C}+\boldsymbol{D}$, Evi knockdown was demonstrated on the protein level in SK-BR-3 cells $(C)$ and their corresponding EV $(D)$ by Western Blotting. $\boldsymbol{E}$, Knockdown of Evi leads to the loss of Wnt5a on MV and Exo from SK-BR-3 and Wnt5a-L shEvi cells (Western Blot). $\boldsymbol{F}$, qRT-PCR: WNT5A mRNA levels are unaffected in SK-BR-3 shEvi cells and only slightly reduced in Wnt5a-L shEvi cells (means \pm SD, $n=3$, *p<0,05). Modified from (Menck et al, 2013).

Surprisingly, when we investigated Wnt5a expression in shEvi cell lines, the protein was significantly diminished and barely detectable inside the cells (Fig. 29A). Evi has often been suggested to exert a chaperone function required for correct folding of Wnt proteins (Ching \& Nusse, 2006). Therefore, we asked if Evi knockdown might lead to increased Wnt5a degradation due to misfolding of the protein. To test this hypothesis we first treated SK-BR-3 and Wnt5a-L shEvi cells with the proteasome inhibitor MG-132 and analyzed cellular viability. Already an incubation with $1 \mu \mathrm{M}$ MG-132 for $6 \mathrm{~h}$ resulted in a beginning reduction of MTT conversion (Fig. 29B+C). Due to the high cytotoxicity of the inhibitor which blocks the proteolytic activity of the 26S proteasome complex (Han et al, 2009), longer incubation periods or higher doses were not possible without inducing apoptosis of the cells. Treatment 
of the shEvi cells with the titrated amount of MG-132 for $6 \mathrm{~h}$ did not augment cellular Wnt5a levels (Fig. 29D) arguing against enhanced proteolytic degradation.
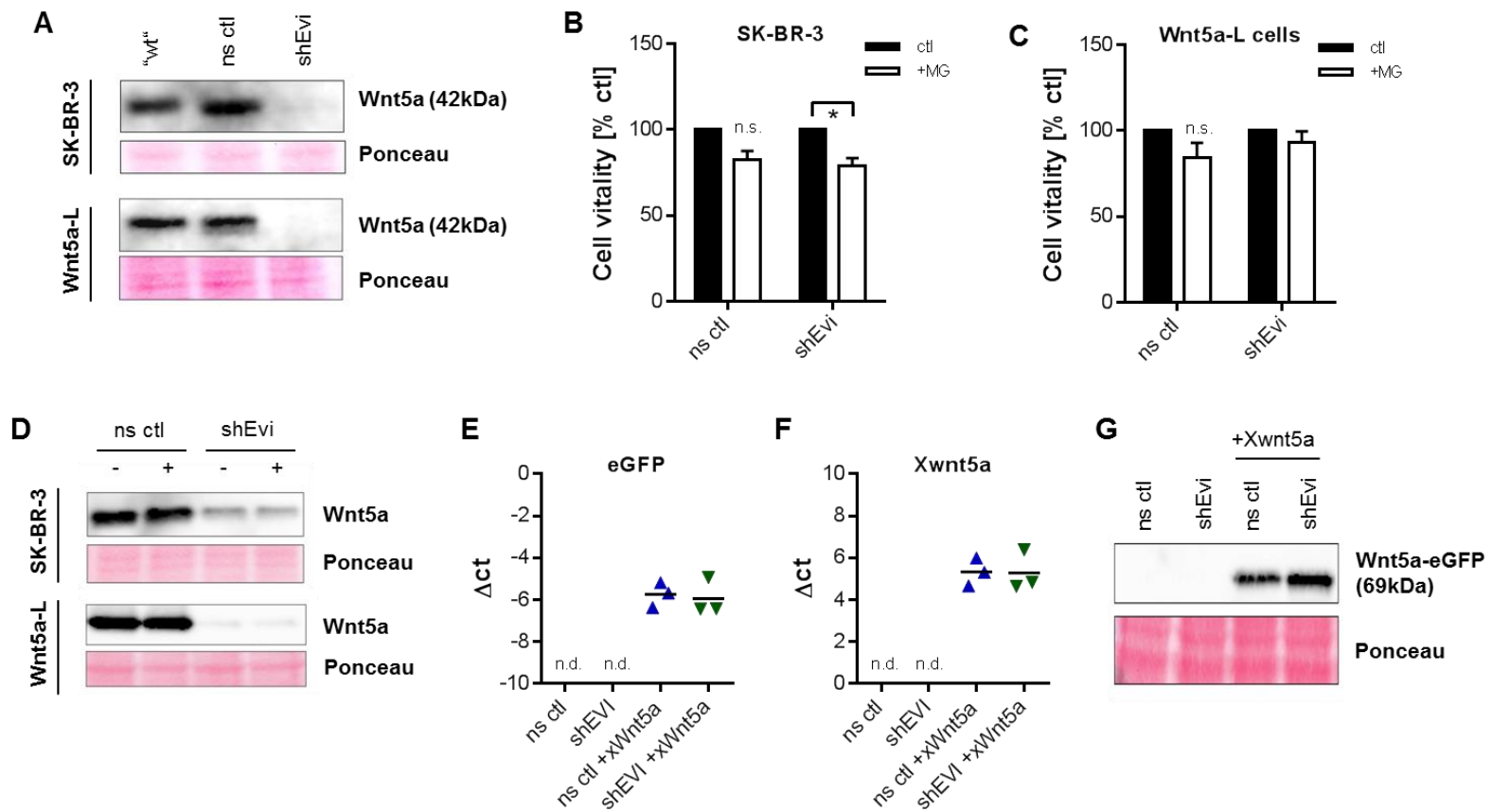

Fig. 29: Implications of Evi knockdown for cellular Wnt5a expression

$\boldsymbol{A}$, Expression of the Wnt5a protein inside shEvi and non-sense control (ns ctl) cells was analyzed by Western Blotting. $\boldsymbol{B}+\boldsymbol{C}$, Cell vitality of SK-BR-3 $(B)$ and Wnt5a-L $(C)$ shEvi cells during $6 \mathrm{~h}$ of treatment with the proteasome inhibitor MG-132 $(1 \mu \mathrm{M})$ was assessed by MTT assay (means $\pm \mathrm{SD}, \mathrm{n}=3$, $* \mathrm{p}<0,05$, n.s. = not significant). $\boldsymbol{D}$, Western Blot: Treatment of shEvi cells with MG-132 $(1 \mu \mathrm{M})$ for $6 \mathrm{~h}$ did not increase cellular Wnt5a expression. $\boldsymbol{E}-\boldsymbol{G}$, SK-BR-3 shEvi and ns ctl cells were transiently transfected with an eGFP-tagged $\mathrm{xWnt5a}$ construct. Expression of the construct was visualized by qRT-PCR with primers directed against eGFP $(E)$ or $\mathrm{xWnt5a}(F)$. $\Delta$ ct values $>12$ were considered as not detectable (n.d.). Expression of the transfected construct can additionally be confirmed by Western Blotting against GFP $(G)$.

Another possibility might be that the Wnt5a antibody is no longer able to detect the protein when it is misfolded due to knockdown of Evi. In order to investigate this in an indirect approach, we transiently transfected SK-BR-3 shEvi cells with a plasmid encoding eGFPtagged Xenopus Xwnt5a (see 2.2.1.3) and analyzed Wnt5a levels in the cells. Expression of the construct was confirmed by qRT-PCR with primers directed against eGFP (Fig. 29E) or Xwnt5a (Fig. 29F) which both could only be detected in the transfected and not in the nontransfected cells. Furthermore, on the protein level expression of the construct inside the cells was visualized by Western Blots directed against GFP (Xwnt5a tagged with eGFP results in a band size of $42+27=69 \mathrm{kDa}$ ). This shows that Xwnt5a is in fact still present in shEvi cells and may even slightly accumulate in comparison to non-sense control cells (Fig. 29G). 
A

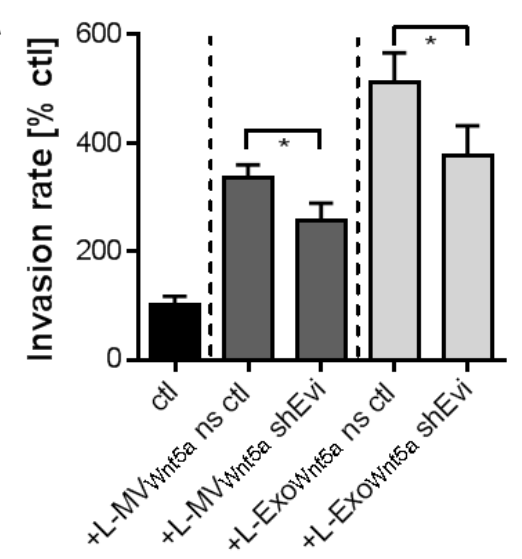

C

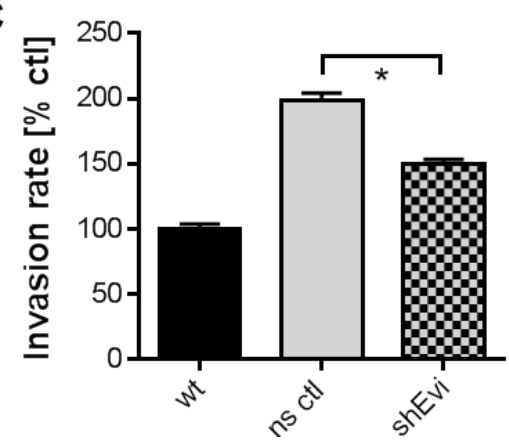

B

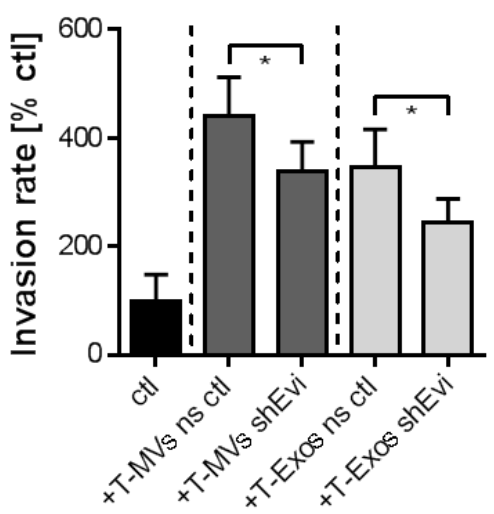

D

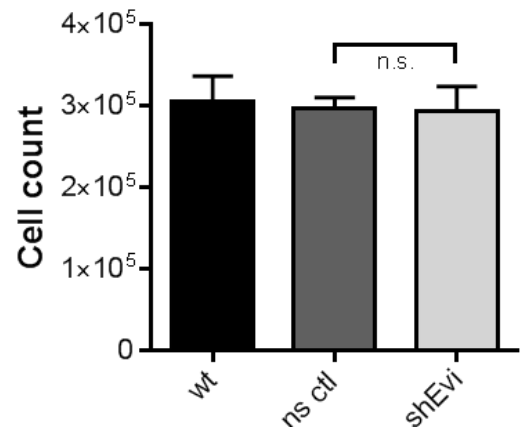

Fig. 30: Knockdown of Evi impairs MV-mediated tumor invasion as well as the pro-invasive phenotype of the tumor cells per se

$\boldsymbol{A}+\boldsymbol{B}$, Microinvasion assays of MCF-7 cells stimulated with $1 \mu \mathrm{g} / \mathrm{ml}$ T-MV/Exo $\mathrm{S}(A)$ or L-MV/Exo $\mathrm{Wnt5a}_{(}(B)$ from shEvi or ns ctl cells (means \pm SD, $\mathrm{n}=3$, ${ }^{*} \mathrm{p}<0,0001$ ). $\boldsymbol{C}$, Microinvasion assay of SK-BR-3 wt, shEvi or ns ctl cells (means $\pm \mathrm{SD}, \mathrm{n}=3,{ }^{*} \mathrm{p}<0,0001$ ). $\boldsymbol{D}$, In parallel to the microinvasion assay, SK-BR-3 cells were seeded on ECMcoated 24-well plates and cell counts were determined after $96 \mathrm{~h}$ (means $\pm \mathrm{SD}, \mathrm{n}=3$, n.s. $=$ not significant). Modified from (Menck et al, 2013).

Since Wnt5a expression was lost on EV from shEvi cells, we asked whether this has any consequences for the pro-invasive function of the vesicles. To test this, we stimulated MCF-7

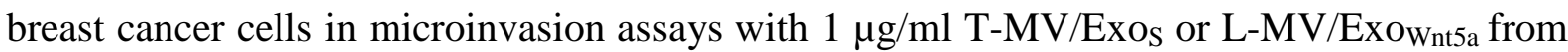
shEvi cells. Wnt5a-negative EV from Evi knockdown cells induced significantly less tumor invasion (Fig. 30A+B) suggesting that it is indeed one of the factors which mediates the proinvasive phenotype of the vesicles in autologous as well as stroma cell-induced tumor invasion. Moreover, Evi knockdown additionally impaired the invasive potential of the SKBR-3 cells per se (Fig. 30C). To confirm that this effect was not due to differences in cell proliferation, we seeded the cells on ECM-coated 24-well plates and counted the number of cells after $96 \mathrm{~h}$. However, there was no significant difference in the proliferation between Evi knockdown and non-sense control cells (Fig. 30D). 


\subsubsection{Zoledronic acid inhibits T-MV uptake into human M $\phi$}

Since EV have been identified as an important tool which tumor cells use to influence the surrounding stroma cells and drive tumor-associated $\mathrm{M} \phi$ into a tumor-supporting phenotype, it might be clinically relevant to inhibit EV-mediated tumor-stroma crosstalk. Zoledronic acid (ZA) is a bisphosphonate and $\mathrm{M} \phi$ inhibitor that is routinely used in the clinic against osteoporosis, where it antagonizes osteoclasts. Recently, it has also been demonstrated to increase the disease-free survival in breast cancer patients in combination with an adjuvant therapy (Gnant, 2009; Gnant, 2011). In mouse models it reduces metastatic tumor burden (Hiraga et al, 2004).

A

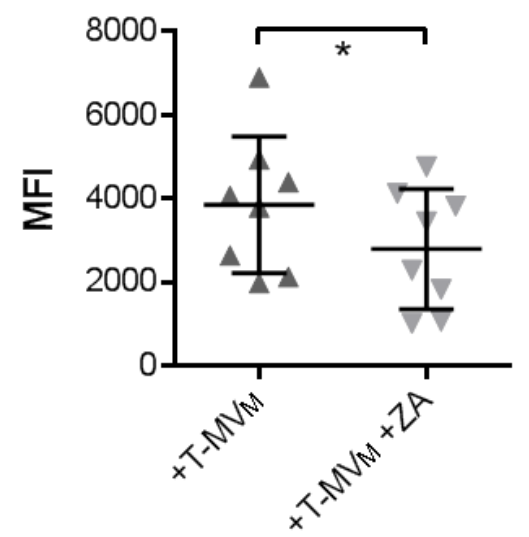

B

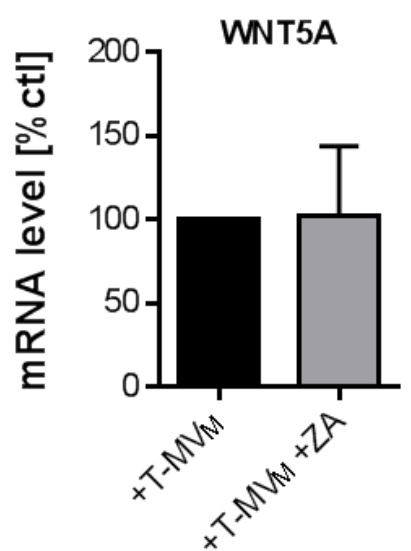

Fig. 31: ZA influences MV-mediated tumor-M $\phi$ crosstalk

$A$, Human $\mathrm{M} \phi$ were pre-treated with $1 \mu \mathrm{M}$ zoledronic acid (ZA) for $48 \mathrm{~h}$ and then stimulated for $24 \mathrm{~h}$ with $5 \mu \mathrm{g}$ PKH26-labeled T-MV $\mathrm{M}_{\mathrm{M}}$. MV uptake was assessed by flow cytometry by comparing the red mean fluorescence intensity (MFI) of the $\mathrm{M} \phi$ (means $\pm \mathrm{SD}, \mathrm{n}=8$, *p $<0,05$ ). $\boldsymbol{B}$, After pre-incubation of $\mathrm{M} \phi$ with $1 \mu \mathrm{M} \mathrm{ZA}$ for $48 \mathrm{~h}$, the cells were stimulated with $25 \mu \mathrm{g} / \mathrm{ml} \mathrm{T}-\mathrm{MV}_{\mathrm{M}}$ for $24 \mathrm{~h}$ and qRT-PCR was carried out from total RNA samples (means \pm SD, $\mathrm{n}=7$ ). Modified from (Rietkotter et al, 2013).

Results from our group suggested that $1 \mu \mathrm{M}$ ZA effectively antagonized M $\phi$-induced MCF-7 invasion (Rietkotter et al, 2013). In search for a mechanism which might explain this phenomenon, we investigated whether ZA interferes with MV-mediated tumor-M $\phi$ crosstalk. As shown in Fig. 31, $1 \mu \mathrm{M}$ ZA impaired $\mathrm{T}-\mathrm{MV}_{\mathrm{M}}$ uptake into $\mathrm{M} \phi$, while there was no influence detectable on MV-mediated WNT5A induction. This is in line with our previous results showing that WNT5A induction is independent of MV uptake (3.2.2) and further suggests that there are additional pro-invasive factors induced in $\mathrm{M} \phi$ by $\mathrm{T}-\mathrm{MV}_{\mathrm{M}}$ stimulation which require an uptake of the vesicles into the cells. 


\subsection{EV in human peripheral blood samples}

The first two parts of this thesis have demonstrated the importance of T-EV, especially T$\mathrm{MV}$, for the establishment of a favorable tumor microenvironment in vitro. They were shown to influence not only the tumor cells themselves in autologous as well as heterologous intercellular communication routes, but also to influence surrounding stroma cells and drive them in a tumor-supporting phenotype. Moreover, one of the pro-invasive factors, HGEMMPRIN, could be identified on T-MV and was established as a sensitive marker for malignant T-MV which discriminates them from T-Exo. We thus wanted to transfer our expertise to a more clinical setting and aimed to establish a reproducible isolation protocol for EV from peripheral blood of cancer patients. Based on additional T-MV-associated markers which we identified in screenings on T-MV from three different breast cancer cell lines in vitro, we evaluated the possible application of these markers to identify circulating T-MV in vivo which is an essential prerequisite for the specific isolation and further characterization of these vesicles.

\subsubsection{Identification of MV-associated tumor markers by in vitro screening of $\mathrm{T}$ - MV}

In order to find proteins which are specifically expressed on T-EV and can be used to identify circulating T-EV in breast cancer patients, we screened three different breast cancer cell lines for the expression of potential tumor-specific proteins on their EV. These cell lines are thought to represent the three breast cancer subtypes which can be classified based on their receptor status as described in chapter 1.1: MCF-7 cells were used as model for hormone receptor-positive (ER+, PR+), SK-BR-3 cells for Her2/Neu-positive and MDA-MB231 for triple-negative (ER-, PR-, Her2-) breast cancers. T-MV and T-Exo were isolated from all three cell lines and characterized for the expression of EMMPRIN, EGFR, EpCAM, Her2 and MUC1 by flow cytometry (Fig. 32 and Fig. 33). These proteins were chosen because they all have been previously reported as prognostic markers in (breast) cancer (Duffy et al, 2000; Fox et al, 1994; Schmidt et al, 2008a; Zhong et al, 2008). 


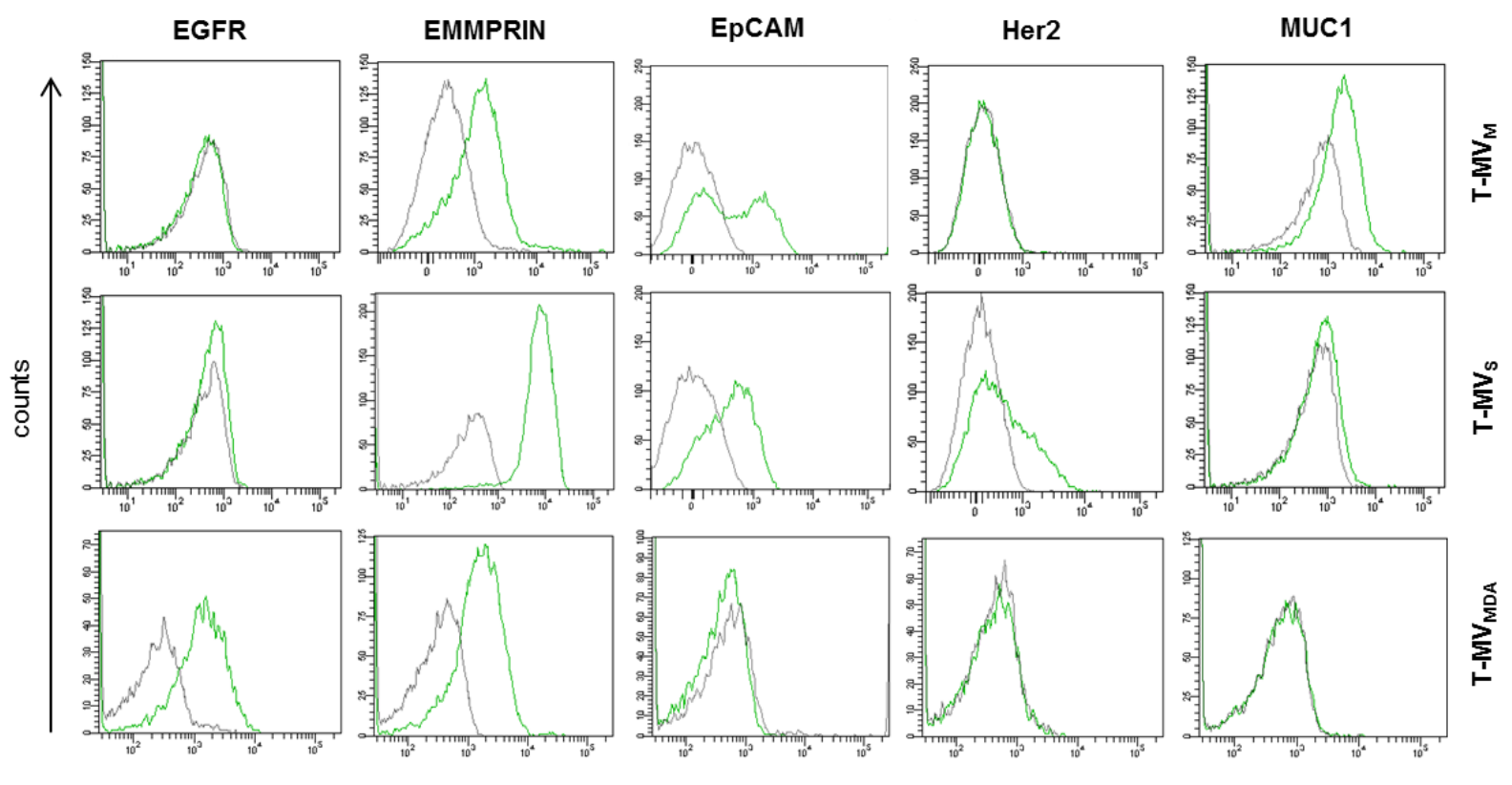

Fig. 32: Tumor marker on T-MV

T-MV $(5 \mu \mathrm{g})$ were stained for the indicated marker proteins and analyzed by flow cytometry. Representative histogram overlays are shown with the specific fluorescent signal in green and the corresponding isotype control in grey.
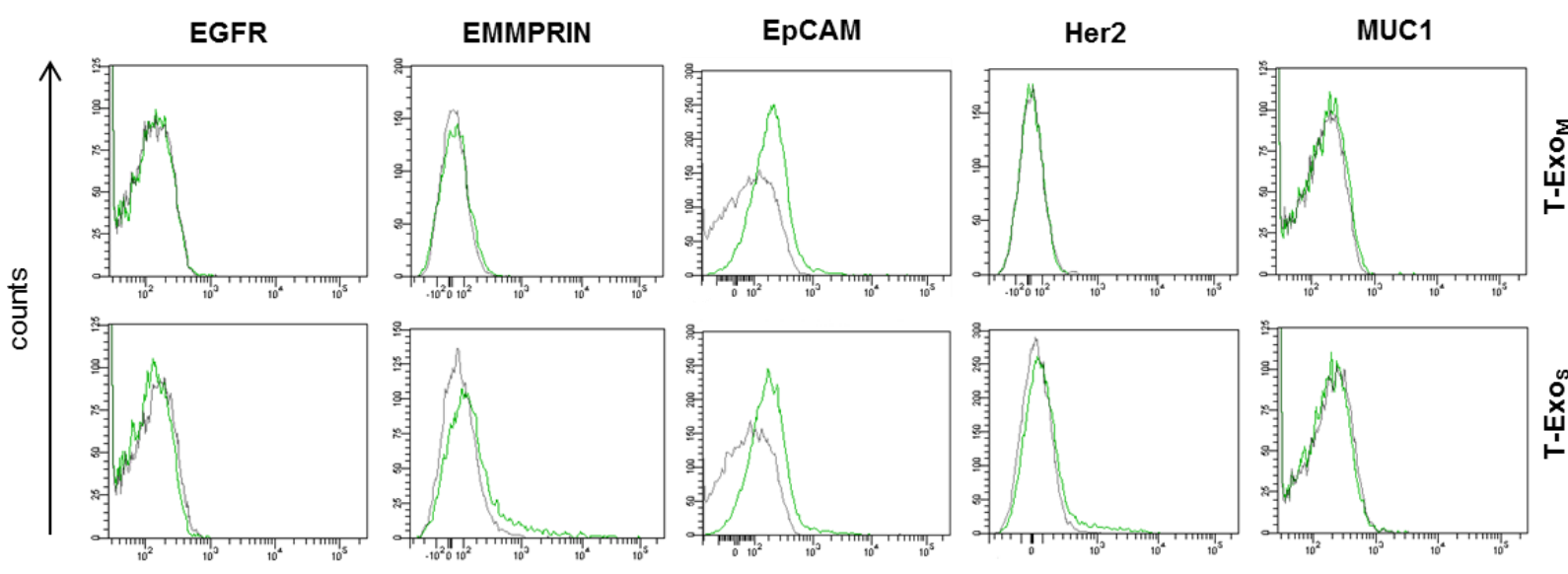

Fig. 33: Tumor marker on T-Exo

T-Exo $(10 \mu \mathrm{g})$ were coupled to latex beads, stained for the indicated marker proteins and analyzed by flow cytometry. Representative histogram overlays are shown with the specific fluorescent signal in green and the corresponding isotype control in grey.

Flow cytometry analysis revealed that EMMPRIN was the only protein which was expressed at high levels on all three MV populations, while EGFR, MUC1 and Her2 were positive on just one. EpCAM, a protein which is routinely used for the detection of circulating tumor cells, was detectable on $\mathrm{T}-\mathrm{MV}_{\mathrm{M}}$ and $\mathrm{T}-\mathrm{MV}_{\mathrm{S}}$, but was lacking on $\mathrm{MV}$ from the triple negative MDA-MB231. Surprisingly, all investigated tumor markers were negative on T-Exo from 
MCF-7 and SK-BR-3 cells so that further characterization of T-ExomDA was not continued. Only EpCAM was expressed in very low levels on T-Exo from both cell lines.

\subsubsection{EpCAM-based MACS of T-EV from human serum samples}

Since we aimed to establish an isolation protocol for T-MV as well as T-Exo from peripheral blood of cancer patients, we selected EpCAM as marker for T-EV since it was the only protein that was expressed on both vesicle populations. To separate T-EV from other EV populations present in blood e.g. EV derived from platelets, leukocytes or red blood cells, we chose an EpCAM-based sorting approach via MACS (see 2.2.4.3) because this method was already described for the sorting of Exo from peripheral blood of ovarian cancer patients (Taylor \& Gercel-Taylor, 2008). Moreover, EpCAM has been previously used to identify circulating tumor cells in metastatic breast cancer (Riethdorf et al, 2007).

First, we tested the method in vitro by spiking $5 \mathrm{ml}$ of human $\mathrm{AB}$ serum samples with a defined amount of $\mathrm{T}-\mathrm{MV}_{\mathrm{S}}$ and titrated the amount of EpCAM-MicroBeads which allows sorting of these vesicles with the highest quality and quantity. Using MACS with different concentrations of EpCAM-MicroBeads, around $30 \%$ of the input T-MV LD columns as determined by Lowry assay (Fig. 34A). There was no significant difference in MV yields between 50, 10 or $5 \mu 1$ EpCAM-MicroBeads used for labeling of T-MV . In order to assess if the MicroBeads also lead to non-specific selection of benign blood-derived MV, human $\mathrm{AB}$ serum was incubated with $10 \mu \mathrm{l}$ EpCAM-MicroBeads alone. This resulted in selection of residual MV showing that to some extent unspecific binding occurs, however, MV counts were considerably higher in the presence of $T-M_{S}$. These results could be further confirmed by flow cytometric counting of MV yields as described in 2.2.4.1.4. Again, there was no significant difference in the addition of 5 or $10 \mu \mathrm{l}$ EpCAM-MicroBeads for sorting and the number of sorted MV was clearly higher when $T-M_{S}$ had been added to the serum samples compared to EpCAM-MicroBeads alone (Fig. 34B). 
A

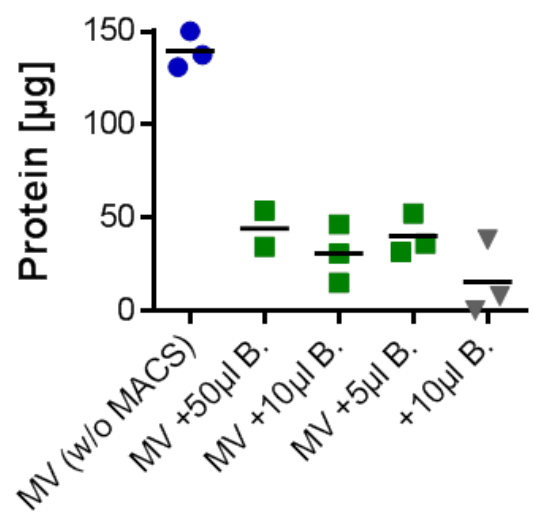

C

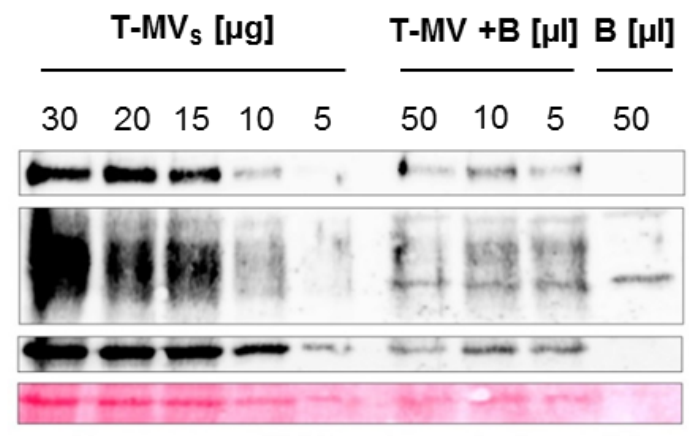

B

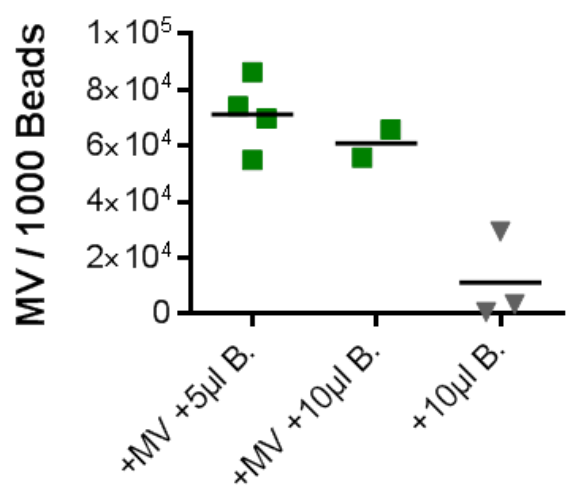

Her2 (185kDa)

EMMPRIN $(50-65 \mathrm{kDa})$

Tubulin $(50-60 \mathrm{kDa})$

Ponceau
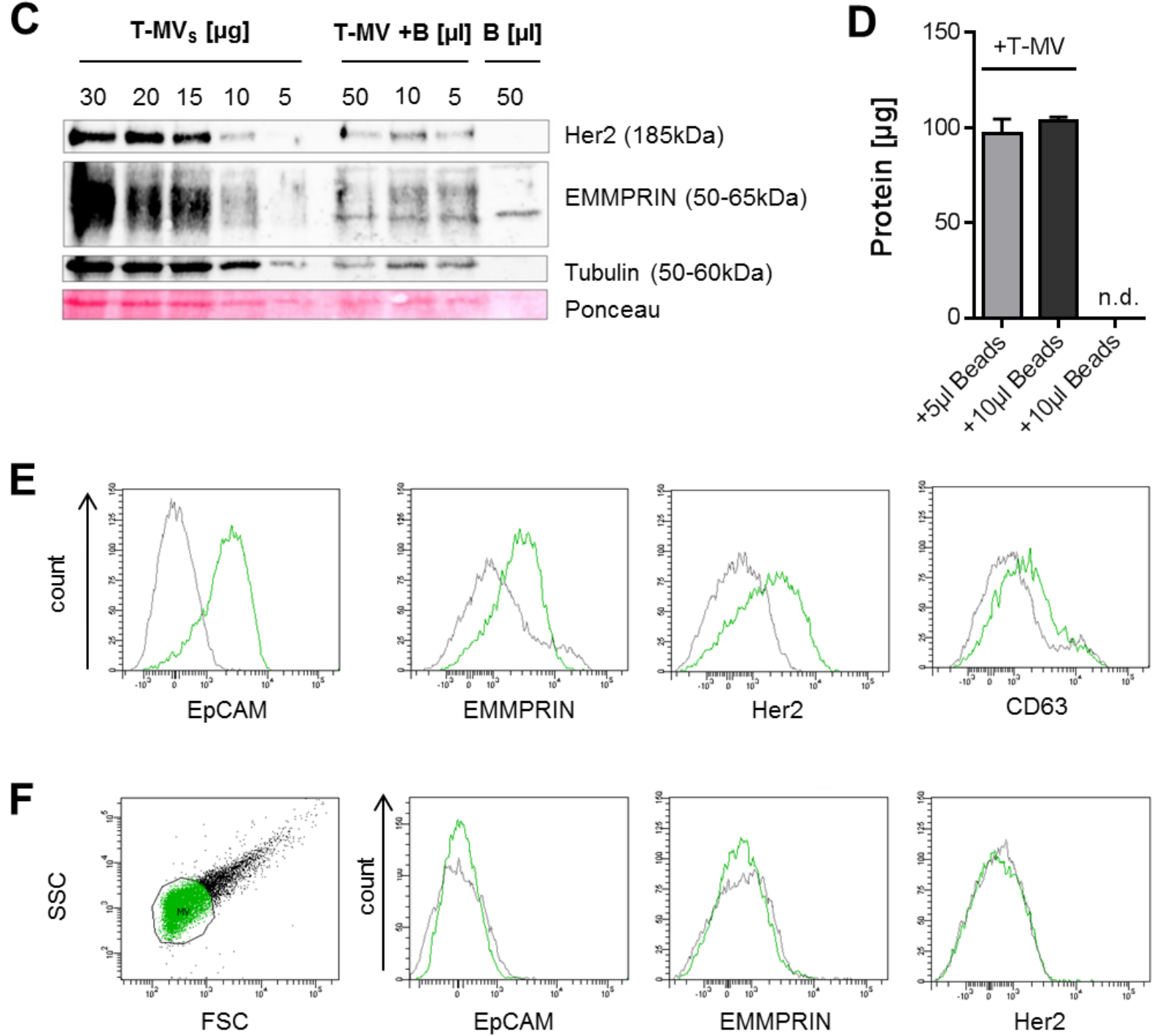

Fig. 34: MACS of $T-M V_{S}$ spiked into human $A B$ serum samples

Human $\mathrm{AB}$ serum samples were spiked with different concentrations of $\mathrm{T}-\mathrm{MV}_{\mathrm{S}}$ and subsequently sorted by MACS. $\boldsymbol{A}+\boldsymbol{B}$, For quantification the amount of column-bound EpCAM+ MV was measured by Lowry assay $(A)$ and MV counts were determined by flow cytometry $(B)$. The indicated lines represent the means of the samples. $\boldsymbol{C} \boldsymbol{+ D}$, Sorted MV were compared to standard samples with known concentrations by Western Blotting using the established T-MV markers $(C)$ and the results of three independent experiments were averaged $(D)($ means $\pm \mathrm{SD}$, n=3, n.d. = not detectable). $\boldsymbol{E}+\boldsymbol{F}$, The column-bound sorted MV (E) as well as the column flow-through (F) were characterized for expression of tumor and MV markers by flow cytometry. Histogram overlays show the specific fluorescent signal in green and the isotype control in grey. 
The vesicles which had been positively selected on LD columns were characterized to confirm the specific binding of $\mathrm{T}-\mathrm{MV}_{\mathrm{S}}$. Western Blot analysis of established T-MV markers (see 3.1.4) demonstrated expression of Tubulin, EMMPRIN as well as Her2 on the sorted vesicles suggesting that they are indeed $\mathrm{T}-\mathrm{MV}_{\mathrm{S}}$ (Fig. 34C). Moreover, the band densities of the samples from the sorted $\mathrm{MV}$ were further compared to a dilution series of $\mathrm{T}-\mathrm{MV}_{\mathrm{S}}$ with known concentrations and thereby allowed quantification of the MV yields by Western Blotting which again confirmed that addition of 5 or $10 \mu 1$ EpCAM-MicroBeads for labeling led to equal MV yields (Fig. 34D). In contrast, putative MV which had been sorted by EpCAM-MicroBeads in the absence of $\mathrm{T}-\mathrm{MV}_{\mathrm{S}}$, were negative for all markers and only a single band of $55 \mathrm{kDa}$ was detected in these samples which corresponds to an unspecific background signal of the MicroBeads (Fig. 34C).

Further characterization of the sorted MV by flow cytometry confirmed a high expression of EpCAM, EMMPRIN and Her2 on the column-bound vesicles while they were negative for the Exo marker CD63 (Fig. 34E). In contrast, none of the three tumor markers was found in the flow-through (Fig. 34F). Addition of $50 \mu \mathrm{l}$ EpCAM-MicroBeads masked most epitopes on the sorted MV and did not allow subsequent characterization by flow cytometry (data not shown) which indicates that as low concentrations of the MicroBeads as possible should be used for MACS. Taken together, the results suggest that indeed the added T-MV $\mathrm{V}_{\mathrm{S}}$ are selected on LD columns and can be eluted from them, although around $70 \%$ of $\mathrm{T}-\mathrm{MV}_{\mathrm{S}}$ are lost during the sorting process, probably due to insufficient elution of the vesicles from the LD columns.
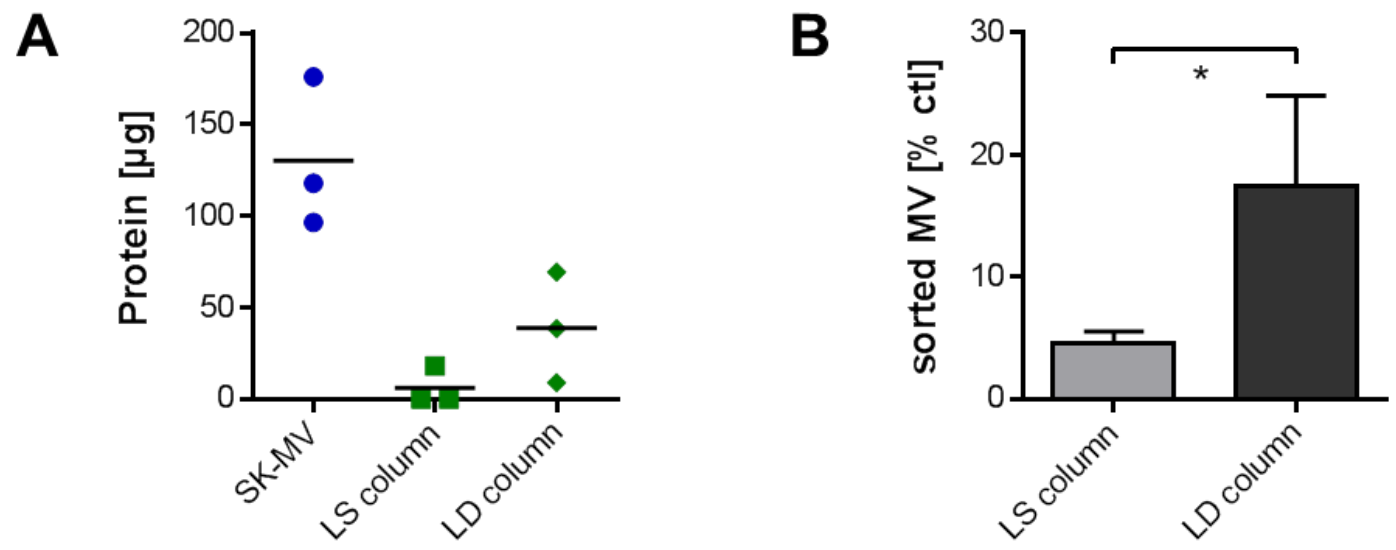

Fig. 35: LD, rather than LS, columns lead to higher MV yields in MACS of T-MV

Human $\mathrm{AB}$ serum samples were spiked with equal amounts of $\mathrm{T}-\mathrm{MV}_{\mathrm{S}}$, labeled with $5 \mu \mathrm{L}$ EpCAM-MicroBeads and sorted on LS or LD columns by MACS. $\boldsymbol{A}$, The yields of column-bound sorted MV were determined by Lowry assay. The lines represent the means of the samples. $\boldsymbol{B}$, MV yields in both samples were further determined by comparing the band densities of three MV markers (EMMPRIN, Her2, Tubulin) in Western Blots analyses with a dilution series of $\mathrm{T}-\mathrm{MV}_{\mathrm{S}}$ with known concentrations (as shown in Fig. 34C). The results for all three markers were averaged and are displayed as percentage of input $\mathrm{T}-\mathrm{MV}_{\mathrm{S}}($ means $\pm \mathrm{SD}, \mathrm{n}=3$, $* \mathrm{p}<0,05)$. 
To test if the yields of sorted T-MV $\mathrm{S}$ can be increased by using LS columns, which are recommended for positive selection by MACS, instead of LD columns which were originally engineered for negative selection, we labeled $\mathrm{T}-\mathrm{MV}_{\mathrm{S}}$ with $5 \mu \mathrm{l}$ EpCAM-MicroBeads and sorted them from human AB serum with LS columns. Surprisingly, yields of positivelyselected MV were considerably lower in Lowry assays (Fig. 35A) as well as flow cytometric MV counting (Fig. 35B) when LS columns were used for selection. This might be explained by the fact that LS columns are specifically designed for positive selection of strongly labeled cells and since MV are much smaller and therefore display a weaker magnetic signal, they possibly pass through the columns.

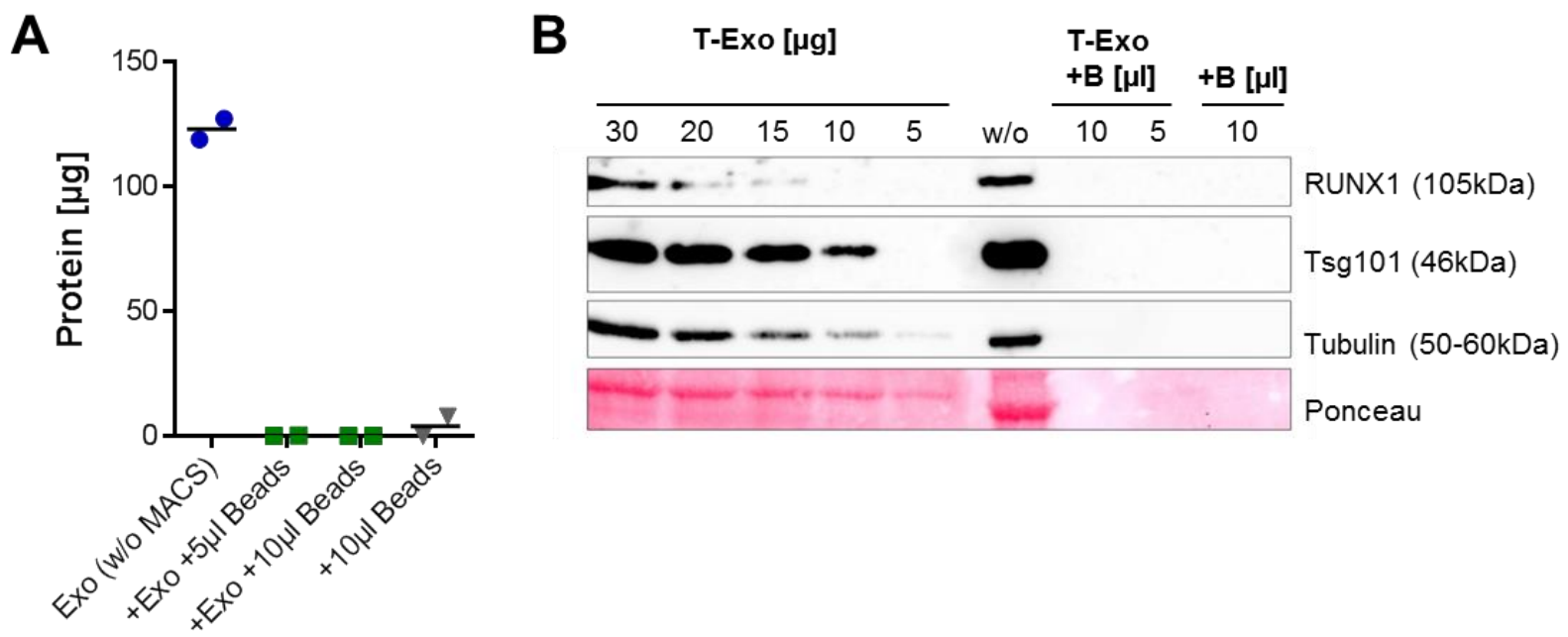

Fig. 36: MACS of T-Exo ${ }_{S}$ spiked into human AB serum samples

Human AB serum samples were spiked with T-Exos, incubated with 5 or $10 \mu 1$ EpCAM-MicroBeads and sorted on LD columns by MACS. $\boldsymbol{A}$, The amount of input (w/o MACS) and column-bound Exo after MACS was determined by Lowry assay $(\mathrm{n}=2)$. The indicated lines represent the means of the samples. $\boldsymbol{B}$, Western Blot analysis: Expression of Exo markers on input T-Exos before (w/o) MACS and column-bound Exo after sorting of T-MV $\mathrm{V}_{\mathrm{S}}$ with 5 or $10 \mu \mathrm{l}$ EpCAM-MicroBeads (=B.). A dilution series of unsorted T-Exos is shown on the left as positive control.

After having established the type of column as well as the concentration of EpCAMMicroBeads which are most applicable for sorting of EpCAM-positive vesicles, we tested the method for its suitability to sort T-Exo. Again, human AB serum samples were spiked with T-

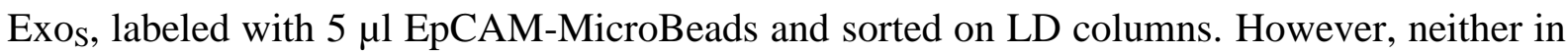
Lowry assays nor in Western Blots we detected any signals in the sorted samples which had been eluted from the columns (Fig. 36). This indicates that EpCAM-based MACS is not suitable for the selection of T-Exo from human serum samples, probably due to the low expression of EpCAM on T-Exo (see Fig. 33). 

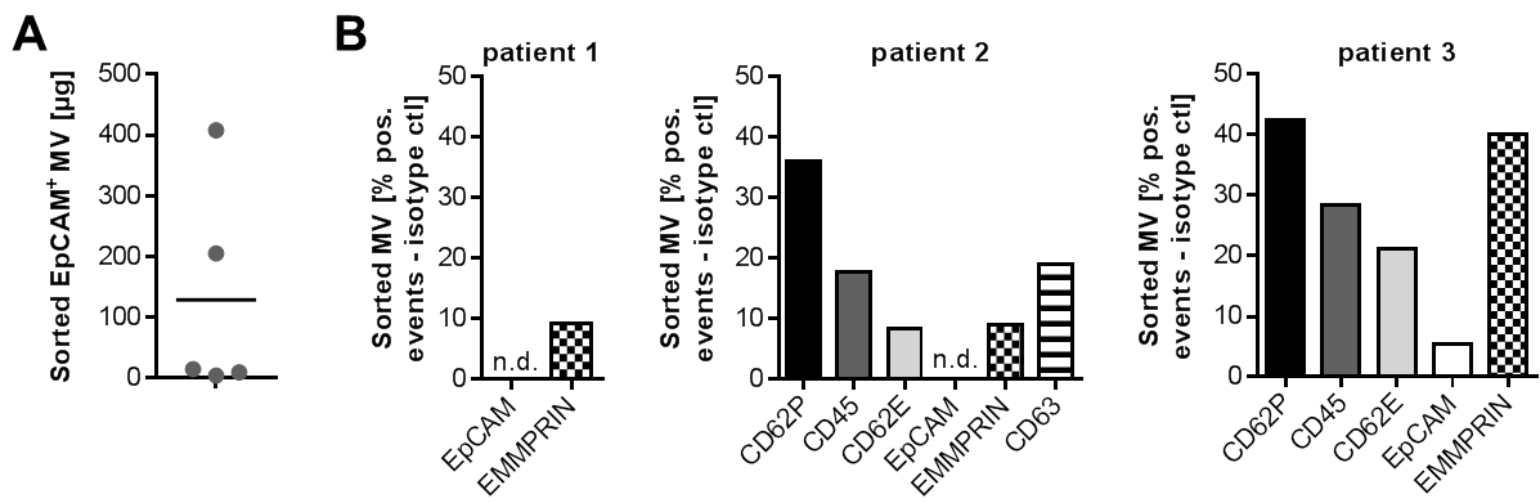

Fig. 37: MACS for MV in serum samples from cancer patients

$A$, Serum samples of five metastatic cancer patients were labeled with 5 or $10 \mu 1$ EpCAM-MicroBeads and sorted for EpCAM-positive MV on LD columns by MACS. The amount of column-bound sorted MV was determined by Lowry assay. The indicated line represents the mean of the samples. $\boldsymbol{B}$, Flow cytometry: Sorted MV from three of the patients were characterized for tumor and blood cell markers. n.d. = not detectable

Nonetheless, since the established approach seemed to be appropriate for EpCAM-based MACS of T-MV, we investigated whether T-MV can also be sorted in vivo from serum samples of cancer patients. All patients were in an advanced disease stage with multiple metastases and were therefore expected to carry a high number of T-EV in their blood. Furthermore, we only included patients who did not receive chemotherapy at the time of sample acquisition to prevent the release of apoptotic bodies from dying tumor cells.

Serum samples $(5-10 \mathrm{ml})$ of five metastatic cancer patients including one patient with primary breast cancer and four patients with primary lung cancer were incubated with up to $10 \mu \mathrm{l}$ of EpCAM-MicroBeads followed by MACS on LD columns. Column-bound vesicles were eluted, subjected to MV isolation and MV yields quantified by Lowry assays (Fig. 37A). In two of the samples from lung cancer patients high MV counts (408 $\mu \mathrm{g}$ and $205 \mu \mathrm{g})$ were measured, whereas the amount of sorted MV was comparably lower in the other three samples with only $14,6 \mu \mathrm{g}, 9,3 \mu \mathrm{g}$ or $3,8 \mu \mathrm{g}$, respectively. The three samples which contained sufficient protein for further analysis were additionally characterized by flow cytometry (Fig. 37B). Although the sorted MV in all samples were positive for EMMPRIN, EpCAM could only be detected in one sample. Moreover, a considerable amount of the vesicles was positive for the platelet marker CD62P as well as the leukocyte marker CD45 and the endothelial cell marker CD62E which suggests that the MV sorted by EpCAM-MicroBeads are not derived from circulating tumor cells, but were false-positively sorted benign blood cell-derived MV. 


\subsubsection{Establishment of a protocol for the isolation of MV from peripheral blood}

Since we were not able to isolate pure T-MV from serum samples by EpCAM-based MACS, we asked if it is possible to detect T-MV in peripheral blood of cancer patients without any previous sorting steps. In order to investigate this question, we first aimed to define which MV populations are found in the blood of healthy individuals and then compare their distribution to samples from cancer patients.

For the analysis of different MV populations in peripheral blood and to ensure that the previously established tumor marker proteins (see 3.3.1) are not expressed on MV from other cell types, we further started to identify markers which are specific for distinct benign MV populations. While CD62E has already been described as a marker for endothelial cellderived MV (Dignat-George \& Boulanger, 2011) and CD235a as specifically expressed on red blood cell-derived MV (Setty et al, 2000), we characterized P-MV and M $\phi-M V$ to define markers for these two MV populations.

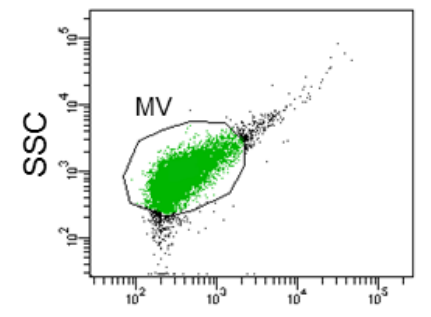

FSC
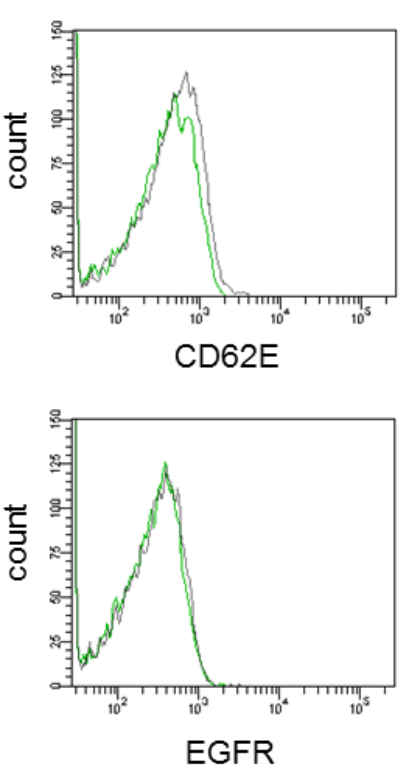

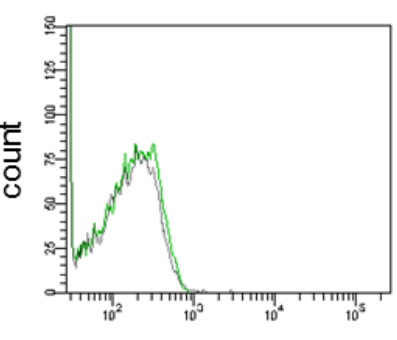

CD11c

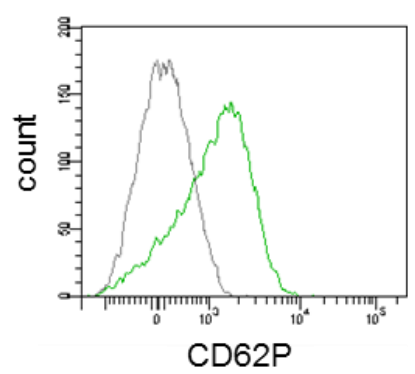

CD62P

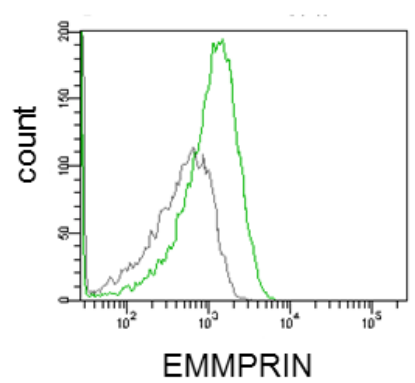

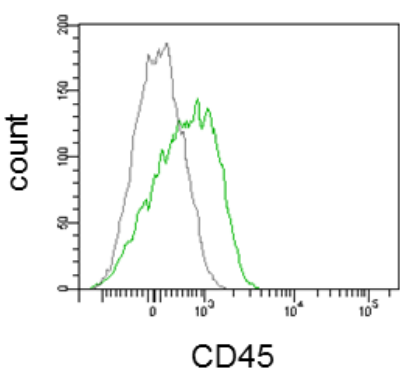
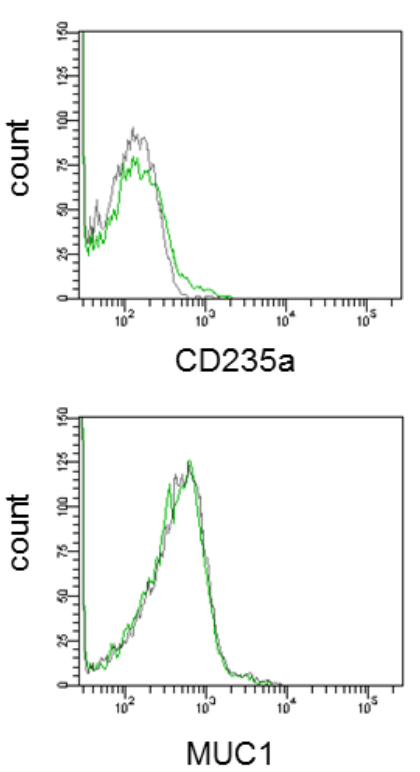

Fig. 38: Characterization of P-MV

P-MV $(5 \mu \mathrm{g})$ were stained for different tumor and blood cell markers and analyzed by flow cytometry. Representative histogram overlays are shown with the specific fluorescent signal in green and the corresponding isotype control in grey. 
As shown in Fig. 38 and Fig. 39, both MV populations were negative for expression of the red blood cell marker CD235a, the endothelial cell marker CD62E as well as the tumor markers EGFR and MUC1. In contrast, they expressed considerable amounts of EMMPRIN which indicates that EMMPRIN expression alone cannot be used for the flow cytometric identification of T-MV in peripheral blood. Furthermore, P-MV characteristically displayed high levels of the cell adhesion molecule CD62P and low expression of CD45, while CD11c, a marker for myeloid cells, was not present. On the contrary, M $\phi-M V$ carried low amounts of $\mathrm{CD} 11 \mathrm{c}$ on their surface and showed a strong expression of CD45. The P-MV marker CD62P was not detectable on M $\phi-M V$. In summary, the results show that CD45, CD62P and CD11c together with the established markers CD62E and CD235a can be used to define subsets of benign MV present in blood. Moreover, all of these markers were negative on malignant breast cancer-derived T-MV as confirmed by flow cytometry (Fig. 40).
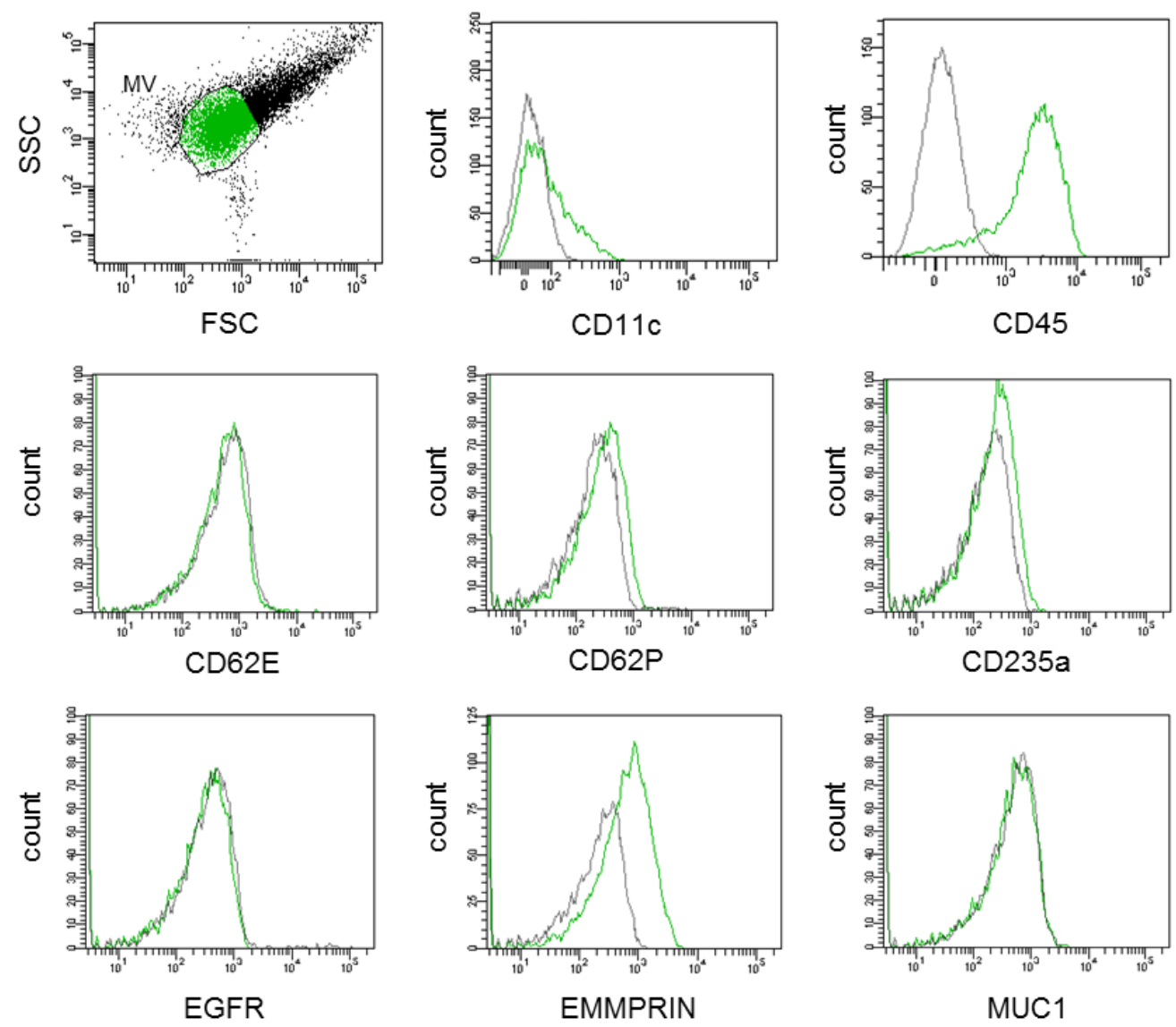

Fig. 39: Characterization of M $\phi-M V$

M $\phi$-MV $(5 \mu \mathrm{g})$ were stained for different tumor and blood cell markers and analyzed by flow cytometry. Representative histogram overlays are shown with the specific fluorescent signal in green and the corresponding isotype control in grey. 


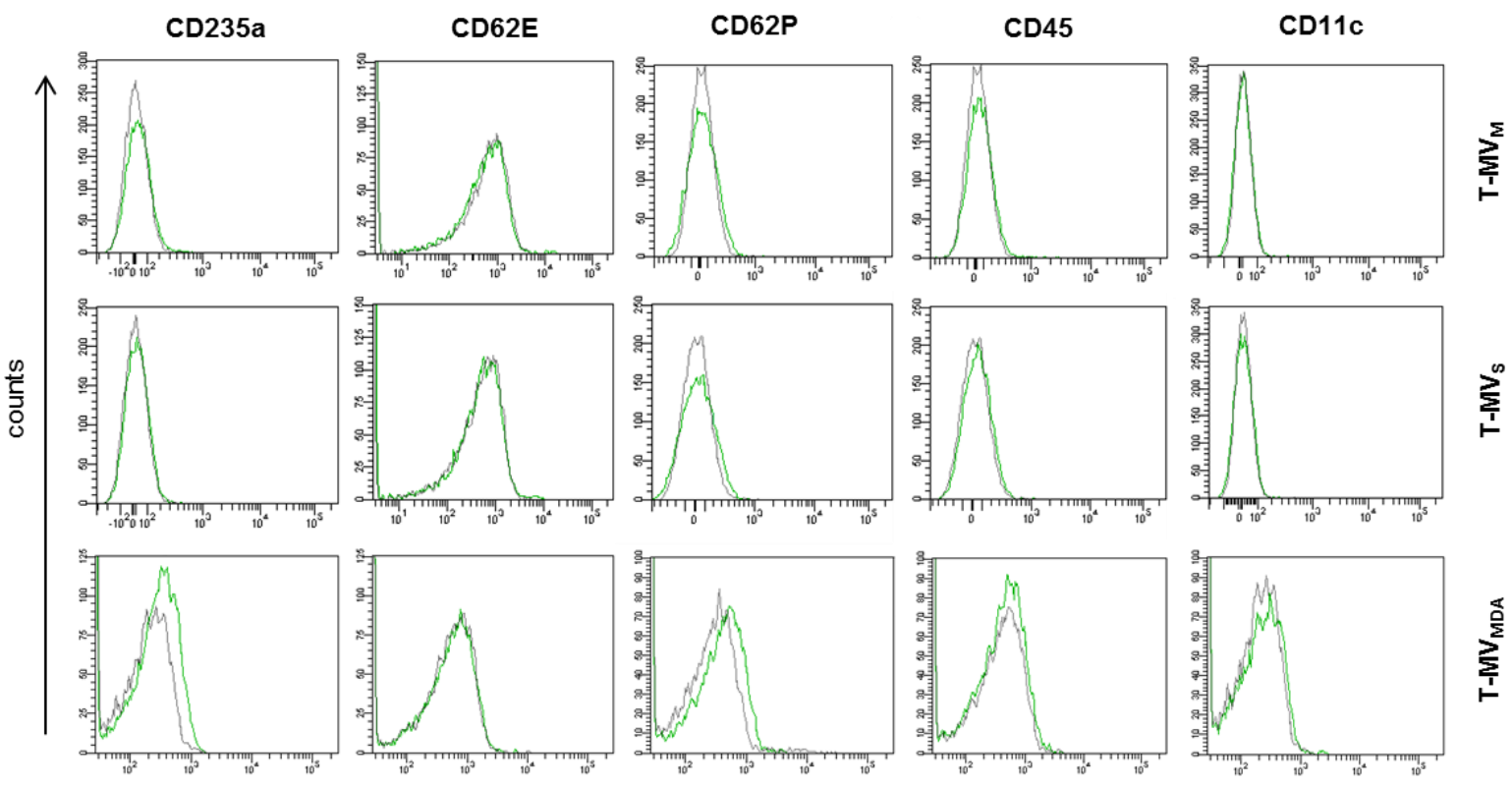

Fig. 40: Blood cell markers on T-MV

T-MV $(5 \mu \mathrm{g})$ were stained for the established blood cell marker proteins and analyzed by flow cytometry. Representative histogram overlays are shown with the specific fluorescent signal in green and the corresponding isotype control in grey.

Regarding methodological issues, the choice of the anticoagulant which is used during collection of the blood samples can have significant effects on subsequently isolated EV. While some publications show a substantial loss of MV in EDTA-anticoagulated blood compared to sodium heparin-treated blood samples (Jayachandran et al, 2012), others discourage the use of heparin-based anticoagulants (Witwer et al, 2013) because heparin was shown to interact with MV (Maguire et al, 2012) and is able to activate platelets (Krauel et al, 2012). For this reason, we analyzed which anticoagulant is most suitable for isolation of total EV from cancer patients.

Peripheral blood was drawn from five cancer patients and either anticoagulated with EDTA or Lithium-heparin ( $\mathrm{Li}-\mathrm{Hep})$. Serum or plasma samples were stored at $-20^{\circ} \mathrm{C}$ for $<14$ days and then thawed for EV isolation (see 2.2.2.1.3) which resulted in visible agglutination in all samples derived from Lithium-heparin-anticoagulated blood. This could neither be prevented by EV isolation directly after blood withdrawal nor by addition of new heparin after each centrifugation step (personal observation). Correspondingly, comparison of EV counts elucidated that MV yields were significantly lower when lithium-heparin had been used as anticoagulant, while Exo, which also tended to aggregate after ultracentrifugation, were slightly increased. In line with this, the number of CD62P- and EMMPRIN-positive MV was decreased in lithium-heparin anticoagulated samples, although this trend did not reach statistical significance. Since both of these proteins had been found in high levels on P-MV 
(see Fig. 38), these findings seem to support the results which demonstrated an activating effect of heparin on platelets, as well as their MV, thereby inducing coagulation. Interestingly, the percentage of CD11c-positive MV was elevated in samples with Lithium-heparin as anticoagulant $(8,0 \%)$ compared to EDTA-coagulated samples $(1,2 \%)$. However, the significance of this observation remains unclear.

A

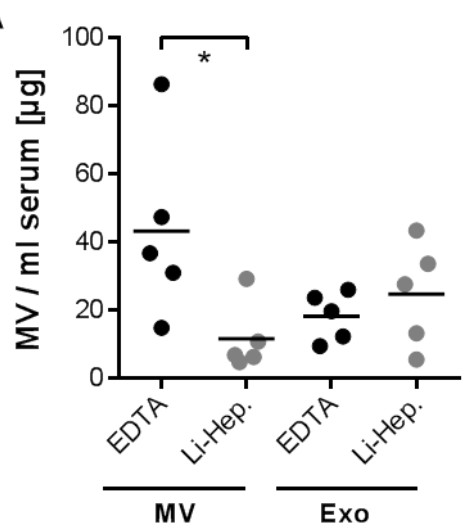

C

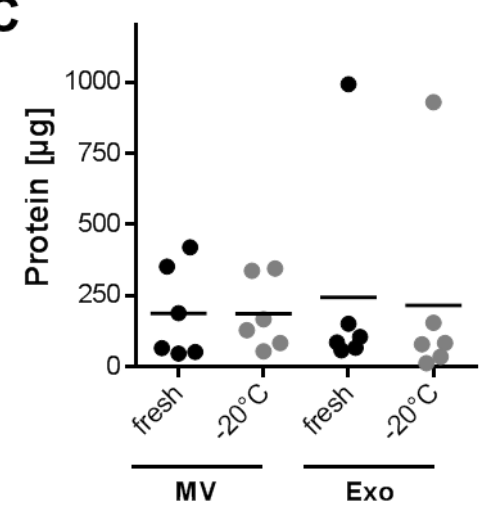

B

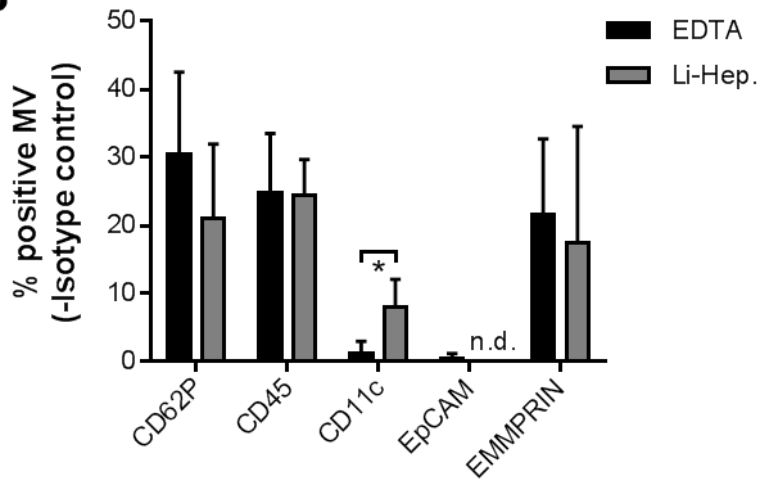

D

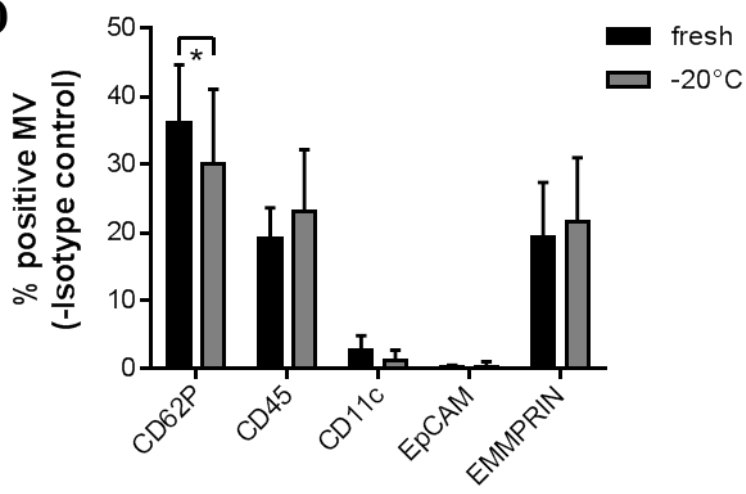

Fig. 41: Comparison of EDTA and heparin as anticoagulants for patient samples

$\boldsymbol{A}-\boldsymbol{B}$, EV isolated from EDTA- or Lithium-Heparin (Li-Hep)-anticoagulated blood were quantified by Lowry assays $(A)(* \mathrm{p}<0,05)$ and MV further characterized for selected blood and tumor cell markers by flow cytometry (B) (means $\pm \mathrm{SD}, \mathrm{n}=5,{ }^{*} \mathrm{p}<0,05$, n.d. $=$ not detectable). $\boldsymbol{C}$ - $\boldsymbol{D}$, EDTA-anticoagulated blood from six cancer patients was either directly or after storage at $-20^{\circ} \mathrm{C}$ for $<14$ days used for $\mathrm{EV}$ isolation. $\mathrm{EV}$ yields were determined by Lowry assays $(C)$ and $\mathrm{MV}$ analyzed regarding expression of selected markers $(D)$ (flow cytometry, means $\pm \mathrm{SD}$, $\left.\mathrm{n}=6,{ }^{*} \mathrm{p}<0,05\right)$. Lines display the mean of the samples.

After having shown that EDTA is more suitable as anticoagulant for patient samples, we asked whether storage of serum samples at $-20^{\circ} \mathrm{C}$ influences EV counts or distribution of MVassociated markers. Therefore, EDTA-anticoagulated blood was drawn from six cancer patients and serum samples either directly subjected to $\mathrm{EV}$ isolation or frozen at $-20^{\circ} \mathrm{C}$ for $<14$ days previous to ultracentrifugation. As shown in Fig. 41C+D, freezing of serum had no significant effects on MV or Exo counts or expression of any of the selected markers for tumor or blood cell-derived MV except a slight decrease in the number of CD62P-positive MV. 


\subsubsection{Isolation of EV from metastatic cancer patients}

Based on the established protocol for the isolation of total MV and Exo from peripheral blood samples, we aimed to investigate whether EV counts as well as the distribution of MV populations differs in cancer patients compared to tumor-free controls. EV were isolated from 21 cancer patients with metastatic disease including patients with primary lung cancer $(n=8)$, breast cancer $(n=4)$, colorectal cancer $(n=4)$, ovarian cancer $(n=1)$, melanoma $(n=3)$ and glioblastoma $(n=1)$. Tumor-free volunteers $(n=14)$ were used as negative controls. The median patient age at the time of sample acquisition was 64 [IQR 59-74] years and the median age of the controls 52,5 [IQR 43,25 - 68,5] years with a p-value of 0,03517 (Wilcox two-sided rank test) indicating that patients were slightly older compared to the controls.
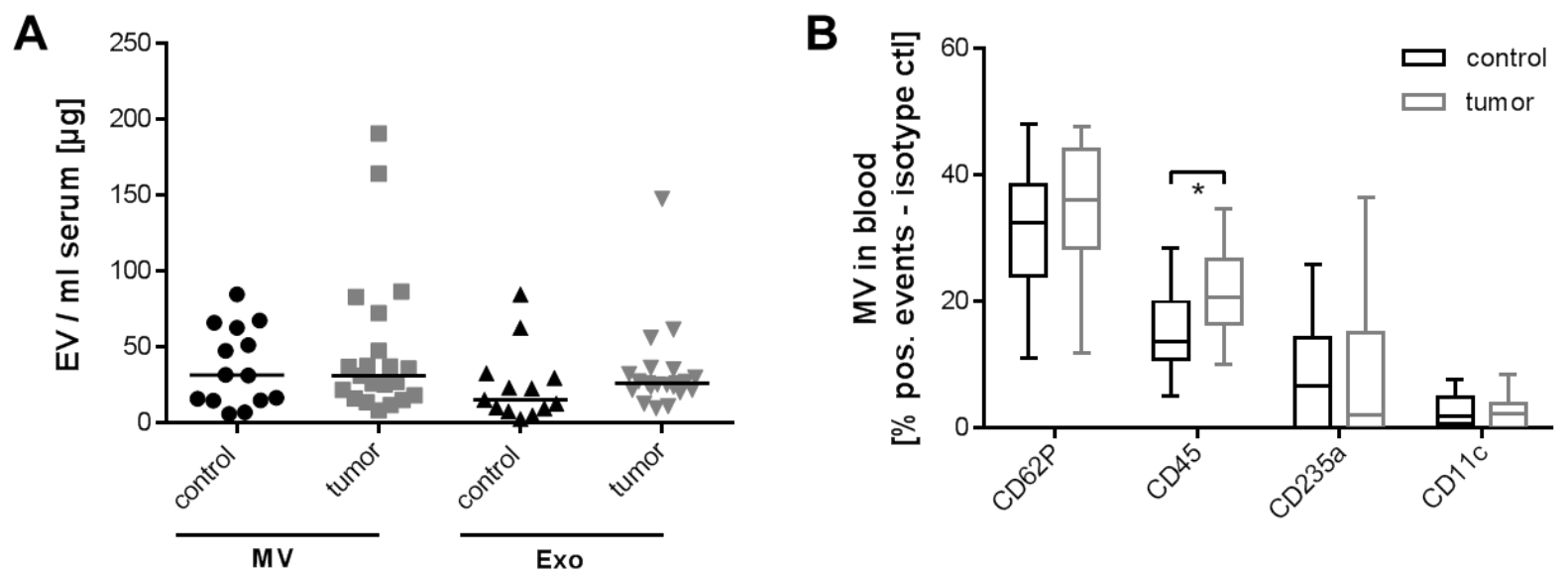

Fig. 42: Isolation and characterization of EV from peripheral blood of cancer patients

$\boldsymbol{A}$, Total EV were isolated from peripheral blood of tumor $(n=21)$ and control $(n=14)$ patients and EV yields determined by Lowry assay. Lines display the median of the samples. $\boldsymbol{B}$, The isolated MV were further characterized for the established blood cell markers by flow cytometry $\left(*_{\mathrm{p}}<0,05\right)$.

Nonetheless, comparison of EV yields between control and patient samples showed no difference in the amount of MV present in blood (patients: median 30,98 [IQR 18,12-47,43], controls: median 31,36 [IQR 14,98-59,63]) with a p-value of 0,6778 (Wilcox one-sided rank test). In contrast, Exo counts seemed to be slightly increased in tumor patients (median 25,98 [IQR 21,64-31,91]) compared to the controls (median 18,81 [IQR 9,57-31,85]), although with a p-value of 0,219 (Wilcox one-sided rank test) this did not reach statistical significance yet (Fig. 42A). Since none of the putative tumor markers was reliably expressed on T-Exo in in vitro screenings (Fig. 33), it was not possible to elucidate if this increase was due to an increased amount of T-Exo in blood. In case of MV, the established markers remained unchanged in both samples for platelet-derived $\left(\mathrm{CD} 2 \mathrm{P}^{+}, \mathrm{p}=0,1632\right)$, erythrocyte-derived $\left(\mathrm{CD} 235 \mathrm{a}^{+}, \mathrm{p}=0,4569\right)$ and myeloid cell-derived $\left(\mathrm{CD} 11 \mathrm{c}^{+}, \mathrm{p}=0,7233\right) \mathrm{MV}$, whereas we 
observed a slight increase in leukocyte-derived $\left(\mathrm{CD} 45^{+}\right) \mathrm{MV}$ in tumor patients compared to the controls ( $\mathrm{p}=0,01297$, all Wilcox one-sided rank test) (Fig. 42B).

Next, we investigated if it is possible to detect T-MV in peripheral blood based on the previously established MV-associated tumor markers (Fig. 43). EpCAM-positive MV were only detected at very low levels (median 1,6\% of total MV) in 15 out of 19 tumor patients and 5 out of 14 control patients. Although this represented a significant increase in patient samples ( $\mathrm{p}=0,01262$, Wilcox one-sided rank test), the low number of EpCAM-positive MV might explain the failure of EpCAM-based MACS for T-MV as described in 3.3.2. In contrast, neither MUC1 nor EGFR were expressed in any of the tested control samples (0/9), but positive in 5 out of 19 patients for MUC1 and 2 out of 18 patients for EGFR. However, this did not reach statistical significance.

A

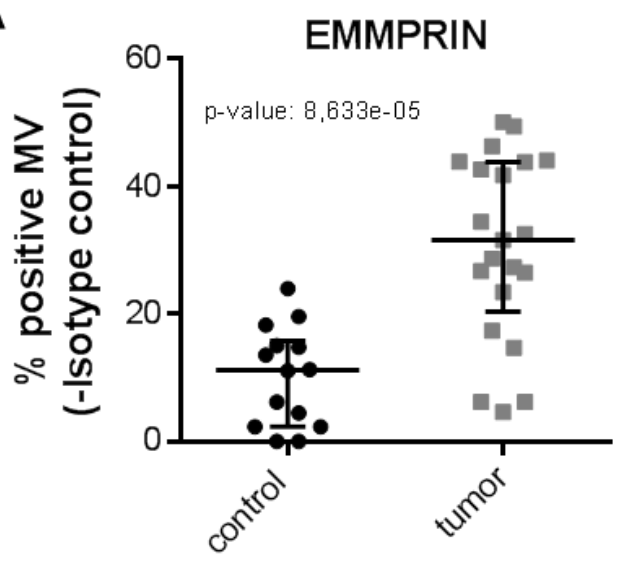

C

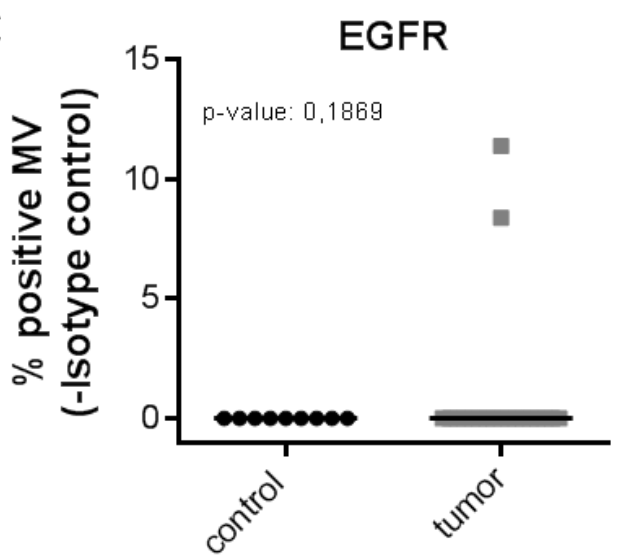

B

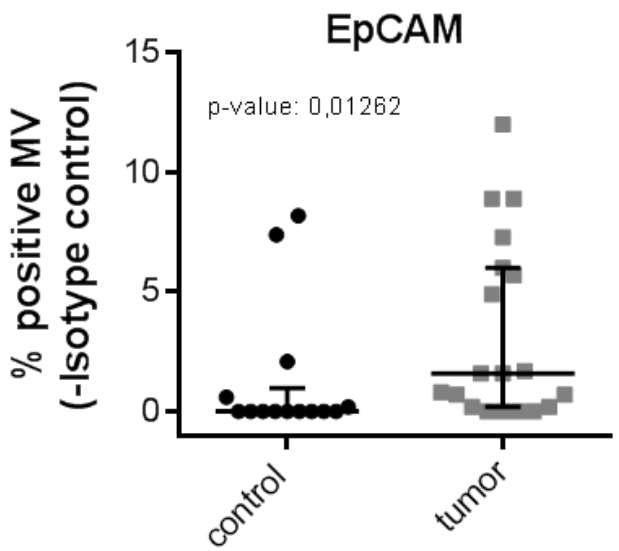

D

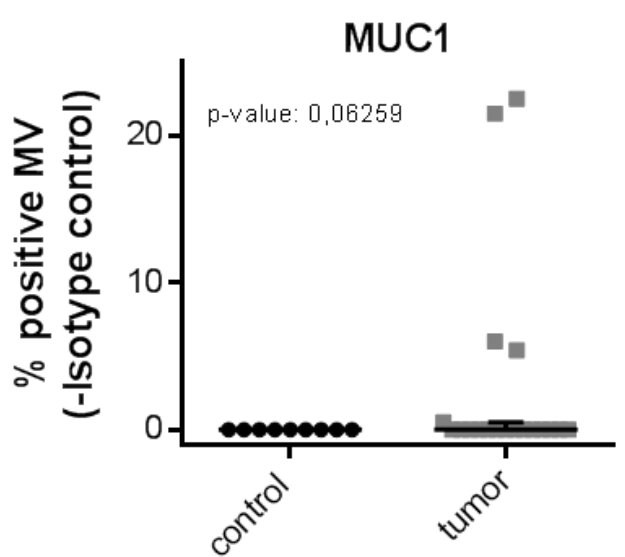

Fig. 43: Tumor markers on MV derived from metastatic cancer patients

Whole MV were isolated from peripheral blood of metastatic cancer and control patients and analyzed by flow cytometry for the expression of the previously defined tumor markers EMMPRIN $(A)$, EpCAM $(B)$, EGFR $(C)$ or MUC1 $(D)$. Lines display the median \pm IQR. Significance was calculated with a one-sided Wilcox rank test. 
Surprisingly, there was a high increase in the number of EMMPRIN-positive MV in tumor patients (median 31,6\% of total MV) compared to tumor-free controls (median 11,2\% of total MV) with a p-value of $8,633 \cdot 10^{-5}$ (Wilcox one-sided rank test). To exclude that this finding was due to the higher age of the patients compared to the control group and further clarify the nature of the EMMPRIN-positive MV, we isolated MV from peripheral blood of five metastatic breast cancer patients for whom we had earlier defined possible tumor markers on breast cancer-derived T-MV in vitro. The median age of the patients at the time of sample acquisition was 51 [interquartile range (IQR): 49,3-57,0] years and the median age of 5 matched tumor-free controls 47 [IQR: 42,5 - 54,5] years with a p-value of 0,5196 (Wilcox two-sided rank test).
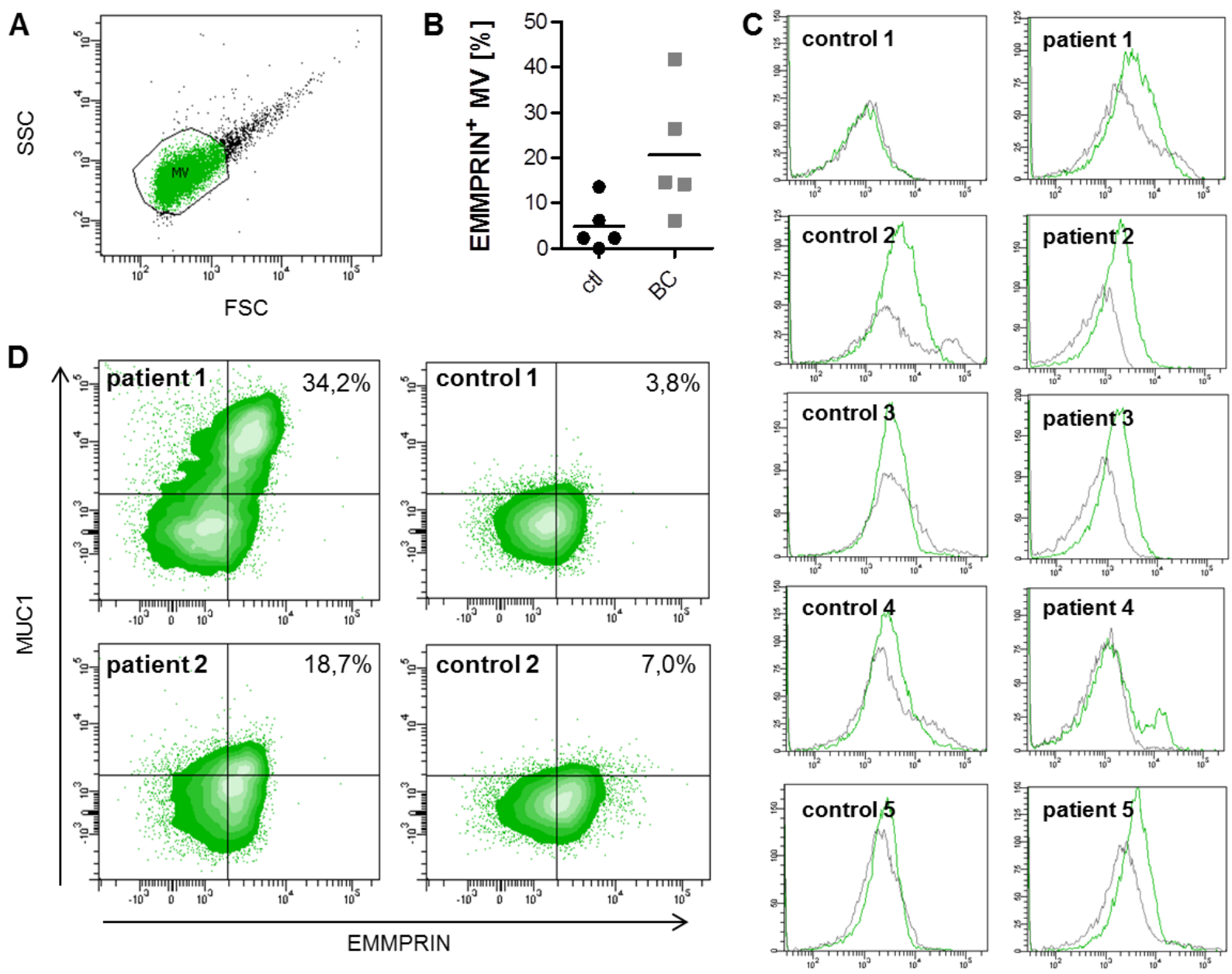

Fig. 44: Detection of T-MV in peripheral blood of metastatic breast cancer patients

$\boldsymbol{A}-\boldsymbol{B}$, Total MV from five metastatic breast cancer patients and matched controls were analyzed by flow cytometry $(A)$ to determine the percentage of EMMPRIN-positive MV (\%positive events - \% isotype control) $(B)$. The line indicates the mean of the samples. $\boldsymbol{C}$, Corresponding histograms for the EMMPRIN expression on total MV from breast cancer patients and controls with the specific signal in green and the corresponding isotype control in grey. $\boldsymbol{D}$, Co-localization of the tumor markers EMMPRIN and MUC1 on MV from two metastatic breast cancer patients compared to matched controls as shown in density plots (flow cytometry). 
The number of EMMPRIN-positive MV in peripheral blood was measured by flow cytometry and was found again to be significantly higher in patient-derived MV samples (median 14,7 [IQR 14,4-26,5] \% of total MV) compared to controls (median 2,3 [IQR 2,3-6,2] \% of total MV) with a p-value of 0,01366 (Wilcox one-sided rank test) as shown in Fig. 44A+B. Since our previous results had demonstrated that EMMPRIN can also be expressed on benign cells, e.g. platelets or myeloid cells (see Fig. 38 and Fig. 39), we investigated whether the EMMPRIN-positive MV additionally express other tumor markers which would argue for their origin from tumor cells. For this reason, we performed double stainings for EMMPRIN and MUC1 by flow cytometry. MUC1, also known as CA 15-3, is often found at high levels in the blood of hormone receptor-positive breast cancers (Park et al, 2012) and was already shown to be present on their released MV (Fig. 32). While two of the further investigated four breast cancer patients were negative for MUC1 on their MV and therefore could not be used for double stainings, the other two patients showed a co-expression of MUC1 with EMMPRIN on $34,2 \%$ or $18,7 \%$, respectively, of all MV present in blood (B). Taken together, the results suggest that T-MV can indeed be found in high numbers in the blood of metastatic cancer patients and can be identified by co-localization of the established markers, including EMMPRIN and MUC1. 


\section{Discussion}

\subsection{The role of $\mathrm{EV}$ in pro-invasive tumor-tumor crosstalk in breast cancer}

\subsubsection{MV and Exo differ in their morphology and protein expression pattern}

So far most functional studies on EV have been conducted with mixed populations of MV and Exo. Accurate discrimination between MV and Exo has only recently gained attention. By using electron microscopy, flow cytometry and Western Blotting we showed that both vesicle populations are indeed morphologically and biochemically distinct and can be isolated separately by differential ultracentrifugation. However, an overlap in the range between 100$200 \mathrm{~nm}$ cannot be excluded and might be responsible for the slight signals of the MVB protein Tsg101 in some MV samples. In correspondence with the known Exo markers summarized in 2002 by Clotilde Théry (Thery et al, 2002), T-Exo specifically expressed the tetraspanin CD63 as well as Tsg101. Moreover, RUNX1 was exclusively present on Exo, although only at very low levels, which confirms previous microarray-based mRNA screenings (Valadi et al, 2007). In contrast, the transmembrane protein EMMPRIN which is overexpressed in many cancers (Nabeshima et al, 2006) was found exclusively on T-MV and could be established as sensitive and specific marker for the discrimination of MV and Exo. This contradicts recent data from Redzic et al who detected EMMPRIN on Exo from MCF-7 and MDA-MB231 cells (Redzic et al, 2013). However, the difference is probably caused by a leaky Exo isolation protocol which allowed the co-purification of MV. This can be concluded from the size reported for their Exo ranging from 50 to $250 \mathrm{~nm}$ (Redzic et al, 2013) thus pointing to the considerable presence of larger EMMPRIN-carrying MV in the sample. Several studies have demonstrated an enrichment of specific membrane proteins on MV. Correspondingly, we observe enrichment of EMMPRIN on MV in comparison to the parental cells. This fits to the hypothesis that MV are shed from lipid rafts which are specialized plasma membrane domains enriched in cholesterol and specific proteins (Lingwood \& Simons, 2010). Cholesterol depletion of the plasma membrane results in decreased MV shedding (Del Conde et al, 2005; Pilzer et al, 2005). Although the mechanism of protein recruitment into lipid rafts is still a matter of debate (Lingwood \& Simons, 2010), EMMPRIN was indeed recently found to be localized at lipid rafts in the plasma membrane of HAT-7 dental epithelial cells (Schwab et al, 2007). 


\subsubsection{T-EV enhance breast cancer progression by stimulating tumor cell invasion in an autologous and heterologous way}

Until now, the role of T-EV in cancer progression has been attributed to their horizontal interaction with surrounding stroma cells or, in the case of T-MV, been seen as a consequence of their procoagulant or matrix-degrading activity (Muralidharan-Chari et al, 2010; VanWijk et al, 2003). In this study we demonstrated for the first time that T-EV also directly enhanced the invasive potential of the tumor cells themselves in an autologous and heterologous stimulation loop. The effect was specific for T-EV since neither the particle-free supernatant nor benign MV influenced tumor invasion. Although T-Exo from breast or colon cancer cell lines or ovarian cancer ascites had already been reported to have a pro-invasive potential (Graves et al, 2004; Higginbotham et al, 2011), this was unknown so far for T-MV. Since the effect was more pronounced for MV, we focused further studies of tumor-tumor interactions on MV.

Several studies have demonstrated that EV are able to transfer malignant characteristics from highly invasive tumor cells to surrounding less malignant subclones (Al-Nedawi et al, 2008; Angelucci et al, 2000; Poste \& Nicolson, 1980). However, benign human mammary epithelial cells remained non-invasive when stimulated with either autologous or heterologous MV indicating that the recipient cell has to be susceptible to transformation to a more invasive phenotype. Moreover, we found that T-MV uptake was an essential prerequisite for their proinvasive effect. Although the mechanism of MV uptake is largely unknown, it was initially hypothesized to occur via two ways (Cocucci et al, 2009): Either via direct fusion with the plasma membrane of the recipient cell as it was already observed for Exo (Montecalvo et al, 2012; Parolini et al, 2009) or via endocytosis. Two of the classical endocytotic pathways involve clathrin- or caveolin-mediated endocytosis (Doherty \& McMahon, 2009). Blocking one of these two pathways was already observed to inhibit uptake of T-Exo from SKOV3 ovarian cancer cells into the same cells (Escrevente et al, 2011) or of T-Exo from U87 MG glioblastoma cells into HUVEC cells (Svensson et al, 2013). In our hands, inhibition of these pathways did not affect the uptake of autologous $\mathrm{T}-\mathrm{MV}_{\mathrm{M}}$ into MCF-7 cells. In line with the study from Kawamoto et al (Kawamoto et al, 2012), inhibition of dynamin blocked MV uptake into breast cancer cells as well as into $\mathrm{M} \phi$ in a dose-dependent manner. This argues for a nonclassical endocytotic pathway involving dynamin (Doherty \& McMahon, 2009). Correspondingly, dynasore was able to reduce MV uptake. However, since it did so only partially, this suggests that additional dynamin-independent mechanisms are involved in this process. Taken together, these results indicate that different uptake mechanisms play a role in 
different cell lines and between different EV populations. Comparative studies are needed to answer this question.

\subsubsection{Glycosylation at N160 and N268 is essential for the pro-invasive effect of MV-associated EMMPRIN on tumor invasion}

Heating of T-MV significantly reduced their effect on tumor invasion suggesting that a major part of their pro-invasive potential is mediated by tumor-supporting proteins which are enriched on T-MV compared to benign MV. However, even after heating T-MV still were able to increase tumor invasion to a certain extent indicating that a contribution of lipids to the pro-invasive phenotype of T-MV cannot be completely excluded.

After having found EMMPRIN enriched on T-MV and considering its important role for proinvasive tumor-stroma crosstalk (Hagemann et al, 2005; Sidhu et al, 2004), we analyzed whether EMMPRIN is also involved in MV-mediated pro-invasive crosstalk between tumor cells. Indeed, shRNA-mediated knockdown of EMMPRIN decreased, but not completely abrogated, the pro-invasive effect of T-MV pointing to the fact that EMMPRIN is one of the proteins that mediate MV-induced tumor invasion. In line with this, the main isoform EMMPRIN-2 was expressed at very high levels in breast cancer cell lines in vitro as well as in human brain metastases from breast cancer patients in vivo.

However, EMMPRIN expression per se is not sufficient to convey a pro-invasive MV phenotype, since the non-invasive hTERT-HME1 displayed the highest expression of the protein on the cells and their released MV. Posttranslational modifications, especially glycosylation, are known to influence the function of EMMPRIN. In this context, Nglycosylation was demonstrated to be required for MMP-2 induction (Sun \& Hemler, 2001). Moreover, a positive correlation between HG-EMMPRIN and MMP-1 expression was found in patients with chronic periodontitis (Wang et al, 2013). In line with this, all pro-invasive TMV expressed a highly-glycosylated form of EMMPRIN with a size of 50-65 kDa, whereas non-pro-invasive MV from platelets and hTERT-HME1 expressed a less glycosylated isoform of only 45-50 kDa which we termed IG-EMMPRIN.

P-MV, which we had identified as non-pro-invasive, displayed only low levels of IGEMMPRIN. Other studies reported that MV isolated from platelets which had been activated with thrombin and/or collagen prior to MV isolation were able to enhance invasion of prostate and lung cancer cells (Dashevsky et al, 2009; Janowska-Wieczorek et al, 2005). Moreover, it was revealed that activation of platelets led to the release of EMMPRIN from intracellular $\alpha$ 
granules which resulted in its increased expression on the cell surface (Schmidt et al, 2008b). Although the glycosylation status of the released EMMPRIN was not determined, it is tempting to speculate that its expression on MV from activated platelets is responsible for their reported pro-invasive effect.

Deglycosylation of $\mathrm{T}_{-} \mathrm{MV}_{\mathrm{M}}$ with PNGaseF resulted in a loss of HG- in favor of IGEMMPRIN which was associated with a decrease in MV-induced tumor invasion. Addition of PNGaseF alone also affected invasiveness of the tumor cells. However, it did not inhibit, but, in contrast, enhance MCF-7 invasion and therefore rather counteracted the observed effect of deglycosylated T-MV. However, since PNGaseF treatment leads to deglycosylation of all glycoproteins present on T-MV, it cannot be ruled out that also other tumor-supporting proteins on T-MV are affected and contribute to the decreased pro-invasive potential. Unfortunately, 2D-DIGE experiments which were carried out to answer this question could not be completed successfully yet.

Nonetheless, the observation that both HG- and IG-EMMPRIN differ in their function has already been made in atherosclerotic lesions where EMMPRIN with a size of $45 \mathrm{kDa}$ was predominantly present in advanced stable plaques, whereas higher glycosylated EMMPRIN of $58 \mathrm{kDa}$ was found in unstable ruptured plaques with ongoing inflammation (Sluijter et al, 2006). Based on our observations we aimed to discriminate IG- and HG-EMMPRIN on the underlying glycosylation pattern to confirm that they indeed represent two different glycoforms. By proteomics approaches we revealed that the two asparagine residues N160 and N268 are glycosylated in HG-, but not in IG-EMMPRIN. Synthetic peptides which cover the glycosylation site at N160 in the first Ig loop were shown to reduce EMMPRIN-mediated MMP induction in fibroblasts (Koga et al, 2011; Sato et al, 2009). Moreover, the first Ig domain seems to be required for homo- and heterophilic interactions which are the basis for induction of MMP-2 (Sun \& Hemler, 2001; Yoshida et al, 2000). In contrast, the second Ig loop which contains the second N-glycosylation site N268 is known to be important for interaction with caveolin-1 (Tang et al, 2004a) which is associated with lipid rafts (Yamaguchi et al, 2009) and could explain the enrichment of EMMPRIN in these specialized membrane domains (Schwab et al, 2007). Furthermore, caveolin-1 was shown to bind to LGEMMPRIN and thereby inhibit its glycosylation and conversion to HG-EMMPRIN which again resulted in reduced MMP induction (Tang et al, 2004a). Altogether, these studies suggest that both extracellular loops with the two N-glycosylation sites N160 and N268 seem to contribute to the pro-invasive function of HG-EMMPRIN on T-MV. 


\subsubsection{The pro-invasive function of EMMPRIN-carrying T-MV is independent of MMP}

Since the enrichment of HG-EMMPRIN on T-MV as well as its glycosylation at the second Ig loop suggested that MV-induced tumor invasion is mediated by induction of MMP in the tumor cells, we aimed to address this question by zymography and qRT-PCR. However, MMP levels remained unchanged in breast cancer cells upon stimulation with MV. Moreover, T-MV carried neither MMP-2 nor MMP-9, although MMP-9 was found to be present on benign $\mathrm{P}-\mathrm{MV}$ and $\mathrm{M} \phi-\mathrm{MV}$ indicating that the presence of MMP is not the critical determinant that confers pro-invasive activity to MV. Although most protumoral effects of EMMPRIN have been associated with regulation of MMP (Muralidharan-Chari et al, 2010), also MMPindependent functions have been demonstrated, such as regulation of angiogenesis (Bougatef et al, 2009) and maintenance of normal cell architecture in Drosophila melanogaster (Curtin et al, 2005). We therefore investigated other known EMMPRIN target genes including $T N F \alpha$, CSF1, VEGF or EMMPRIN itself (Schmidt et al, 2008b; Seizer et al, 2010; Tang et al, 2005). However, they all remained unchanged in MCF-7 upon T-MV stimulation. Upstream of the induction of EMMPRIN target genes, we detected phosphorylation of p38/MAPK occurring in MCF-7 $1 \mathrm{~h}$ after stimulation with T-MV. Phosphorylation of $\mathrm{p} 38$ could be blocked by the specific p38 inhibitor SB-203580 and resulted in a significant decrease in MV-mediated tumor invasion. This argues for an involvement of p38 in the signal transduction after MV binding and is in line with a study from Lim et al who demonstrated p38 activation in 16-Lu lung fibroblasts after stimulation with EMMPRIN (Lim et al, 1998).

\subsection{The role of $\mathrm{EV}$ in Wnt5a induction and transport in $\mathrm{M} \phi$-induced breast cancer invasion}

\subsubsection{T-MV drive M $\phi$ in a tumor-supporting phenotype by inducing Wnt5a}

Previously, our group had demonstrated that tumor-associated $\mathrm{M} \phi$ can enhance breast cancer invasion and progression (Hagemann et al, 2004; Pukrop et al, 2006). In the present study we demonstrated that $\mathrm{EV}$, and not soluble factors, are responsible for this pro-invasive tumorstroma crosstalk. Interestingly, EV stimulation did not induce the expected phenotype switch in M $\phi$ characterized by a typical M2 profile of secreted cytokines. Instead, it led to upregulation of $\mathrm{M} \phi-\mathrm{Wnt5a}$ which was characteristic for $\mathrm{EV}$ from malignant cells, since it was not observed with benign P-MV. Wnt5a is a factor that is typically induced in myeloid cells 
upon inflammatory responses (Blumenthal et al, 2006; Pereira et al, 2008). Although its induction was reported to occur via activation of the $\mathrm{Wnt} / \mathrm{Ca}^{2+}$ pathway (Pereira et al, 2008; Valencia et al, 2011), we did not observe activation of CamKII, PKC or JNK signaling in M $\phi$ after MV stimulation. This indicates that none of the two known non-canonical Wnt pathways was involved in Wnt5a induction.

Several studies demonstrated that Wnt5a influences dendritic cell differentiation. This results in a tolerogenic phenotype with an impaired ability to react properly to TLR stimulation or to promote Th1-directed immune responses (Bergenfelz et al, 2012; Valencia et al, 2011). Moreover, Wnt5a, but not Wnt3a, was found to induce expression of anti-inflammatory cytokines and block differentiation of monocytes to pro-inflammatory M1 M $\phi$ which significantly reduced their cytotoxic anti-tumor response (Bergenfelz et al, 2012). Based on these findings it has been suggested that Wnt5a is part of a negative feedback loop (Bergenfelz et al, 2012) that is thought to prevent an excessive immune reaction after TLR stimulation. Thus, it seems that a physiologic phenomenon is exploited by the tumor cells for reprogramming tumor-associated $\mathrm{M} \phi$ to support tumor progression.

T-MV-mediated WNT5A induction in M $\phi$ was shown to be independent of MV uptake which suggests that MV interact with a still unknown receptor on the M $\phi$ surface to induce p38 signaling and WNT5A transcription. Similar observations have been made for interactions of platelet- or neutrophil-derived MV which bound to specific target cells including monocytes or platelets and induced specific signaling responses (Gasser et al, 2003; Losche et al, 2004; Pluskota et al, 2008). The fact that the Wnt-inhibitor DKK-1 was able to block p38 activation and subsequent WNT5A induction points to Wnt ligands as putative mediators of receptor activation. On the other hand, ZA which inhibits $\mathrm{M} \phi$-assisted tumor cell invasion (Rietkotter et al, 2013) was found to decrease MV uptake into M $\phi$ without affecting WNT5A expression. One explanation for ZA influencing MV uptake is its inhibitory effect on small G proteins which are involved in almost all endocytotic pathways, especially the ingestion of particles into phagocytes (Doherty \& McMahon, 2009). Studies from Luckman et al revealed that nitrogen-containing bisphosphonates including ZA can inhibit enzymes of the mevalonate pathway which results in impaired protein prenylation, an important posttranslational modification for the activity of small G proteins (Luckman et al, 1998a; Luckman et al, 1998b). Since small $G$ proteins also include proteins of the Arf family and Arf6 has been shown to regulate MV shedding (Muralidharan-Chari et al, 2009), it would be interesting to investigate whether ZA additionally impairs the release of MV from tumor cells or M $\phi$. This could provide an additional explanation for its effect, however, it has not been tested yet. 
Taken together, the results imply that T-MV influence human $\mathrm{M} \phi$ in at least two ways: 1) by binding to a membrane-associated receptor which activates p38 signaling and leads to induction of WNT5A transcription, 2) by horizontal transfer of tumor-promoting proteins or nucleic acids which require to be taken up into the $\mathrm{M} \phi$ in order to fulfill their function.

\subsubsection{Wnt5a is transported on MV and supports tumor progression}

The augmented WNT5A transcription was translated into increased expression of the Wnt5a protein in T-MV-stimulated M $\phi$. Earlier in vivo studies had already identified Wnt5a-positive M $\phi$ in immunohistochemical tissue stainings of colon cancers (Smith et al, 1999) as well as breast cancers where they were predominantly localized at the invasive front of the tumor (Pukrop et al, 2006). Furthermore, Wnt5a has been detected at the leading edge of basal cell carcinomas in the tumor cells as well as in associated fibroblasts (Pourreyron et al, 2012). Therefore, one could hypothesize that stroma cells, especially $\mathrm{M} \phi$ recruited to the tumor site, are primed by T-MV to express and release Wnt5a which then acts back on the tumor cells and enhances tumor invasion into the surrounding non-malignant tissue. This is in accordance with our finding that Wnt5a which was exported on $\mathrm{M} \phi-\mathrm{EV}$ in response to T-MV stimulation was transported back to the tumor cells where it increased the invasiveness of the tumor cells.

Until recently, Wnt proteins have been regarded to act predominantly as secreted proteins. However, we found that MV and Exo, although they are two biological distinct populations of extracellular vesicles, both carried Wnt5a produced by stimulated $\mathrm{M} \phi$. Association of the Wnt5a protein with MV, which was unknown so far, could additionally be confirmed for MV of the Wnt5a-positive breast cancer cell line SK-BR-3 as well as for murine Wnt5aoverexpressing L cells. By electron microscopy, we revealed that Wnt5a was not delivered inside the vesicles, but was associated with the membrane of M $\phi-\mathrm{MV}$. This can be partly explained by an unspecific binding of Wnt proteins to biological membranes due to their lipid moieties. In this context, studies from Morrel et al demonstrated a stabilization and potentiation of the activity of canonical Wnt3a when bound to liposomes (Morrell et al, 2008). Moreover, association of Wnt proteins with EV is thought to be important for their long-range signaling capacity as well as in the formation of gradients which are essential for the activity of Wnt proteins (Solis et al, 2013). For instance, in in vitro migration assays, a constant Wnt5a concentration was shown to inhibit cell migration, whereas a Wnt5a gradient induced exactly the contrary (Pourreyron et al, 2012). This might, to some extent, explain the varying results concerning the role of Wnt5a in tumor progression. Nonetheless, Wnt 
gradients seem to reflect the in vivo situation more accurately since Wnt5a gradients could already be visualized in non-melanoma skin cancers (Pourreyron et al, 2012).

Although there was some unspecific association of Wnt5a with EV in our study, we showed that it was predominantly bound via specific association to the seven-pass transmembrane protein Evi. This was surprising since an Evi-dependent export had only been demonstrated so far for Exo and the canonical ligand Wnt3a (Gross et al, 2012). It was unknown for MV and non-canonical Wnt ligands like Wnt5a. Knockdown of Evi led to an almost complete loss of Wnt5a on MV as well as Exo. In accordance with the results from Gross et al we expected that this should lead to an accumulation of Wnt5a inside the cells (Gross et al, 2012). However, after Evi knockdown we could no longer detect the protein inside the cells. Inhibition of protein degradation with the proteasome inhibitor MG-132 at the highest tolerable dose did not augment Wnt5a expression, indicating that an increased degradation is not the explanation for the observed effect. Therefore, we hypothesized that the putative loss of Wnt5a was probably due to conformational changes of the protein which no longer allowed its detection with the used antibodies. This observation supports the often proposed, but until now unproven, theory that Evi is not only important for the export of Wnt proteins, but also has an important chaperone function. To further corroborate this assumption, we assessed Wnt5a distribution by transfecting SK-BR-3 shEvi cells with an eGFP-tagged Xwnt5a fusion protein. Thus, Wnt5a could be visualized by antibodies directed against GFP. Using this approach, Wnt5a was not only detectable in shEvi cells, but its levels even seemed to be slightly compared to non-sense controls. This may indeed point to an accumulation of the protein inside the cells due to the impaired export on EV.

Wnt5a-positive EV released from stroma cells, including $\mathrm{M} \phi$ and Wnt5a-L cells, were shown to highly increase the invasive potential of human breast cancer cells. Again, the particle-free supernatant had no effect, although it still contained all soluble mediators which our group had previously identified as essential for tumor invasion (Hagemann et al, 2004). Interestingly, loss of Wnt5a on EV due to the knockdown of Evi was found to significantly decrease the invasive potential of EV derived from Wnt5a-L cells. The same effect was observed for the MV-induced heterologous invasion of MCF-7 cells stimulated with T-MV from SK-BR-3 shEvi cells. These results indicate that in MV-induced human breast cancer invasion Wnt5a acts as tumor-promoting factor in tumor-tumor as well as tumor-stroma interactions. However, loss of Wnt5a did not completely revert the pro-invasive phenotype of tumor- and stroma-derived vesicles which shows that additional pro-invasive factors like e.g. HG-EMMPRIN are present on the vesicles and contribute to their tumor-supporting function. 
After uptake, MV-bound Wnt5a was detectable within the tumor cells. However, the pathways activated by the MV-delivered Wnt5a in the tumor cells are still unclear. Previous studies with MCF-7 cells reported an activation of JNK signaling in the tumor cells after stimulation with rhWnt5a, whereas PKC was not involved (Pukrop et al, 2006). In contrast, Wnt5a-induced migration and invasion of melanoma cells was mediated by the $\mathrm{Wnt} / \mathrm{Ca}^{2+}$ pathway with enhanced PKC phosphorylation (Weeraratna et al, 2002). This could be antagonized by blocking the Wnt5a receptor Frizzled 5 pointing to a receptor-ligand-mediated effect. Additionally, also the involvement of canonical Wnt signaling for the protumoral effect of Wnt5a is under discussion: On the one hand, Wnt5a was found to increase invasiveness of pancreatic cells by inducing epithelial-mesenchymal-transition (EMT) in the cells in a B-catenin-dependent way (Bo et al, 2013). On the other hand, our own results showed that, although a functional ß-catenin pathway was required, canonical Wnt signaling was not activated in MCF-7 cells under co-culture conditions with M $\phi$ (Pukrop et al, 2006).

Taken together, our observation that breast cancer cells can specifically reprogram $\mathrm{M} \phi$ into a tumor-supporting phenotype with the help of T-EV seems to be in accordance with the proposed "Seed and Soil" hypothesis formulated by Stephen Paget: Breast cancer cells tend to metastasize to specific organs including liver, lungs, bone and brain. Each of these organs harbors a specific $\mathrm{M} \phi$ population: Kupffer cells in the liver, alveolar macrophages in the lungs, osteoclasts in the bone as well as microglia in the brain. One possible explanation for the organ tropism in breast cancer therefore might be that these specialized M $\phi$ populations are prone to be influenced by the tumor cells. After reprogramming, they support the tumor cells in the establishment of a premetastatic niche via the release of Wnt5a-positive proinvasive $\mathrm{EV}$, thereby promoting metastasis formation in the respective tissue.

\subsection{EV in peripheral blood of metastatic cancer patients}

\subsubsection{Isolation of T-EV from human serum samples}

In the last years EV have been increasingly recognized as potential cancer biomarkers which deliver valuable information about the underlying tumor biology. EV can be obtained easily by non-invasive methods from body fluids such as blood or urine which is especially important for cancers where tissue samples are not easily accessible e.g. pancreatic cancer or glioblastoma (D'Souza-Schorey \& Clancy, 2012). In order to establish a protocol for the specific isolation of T-EV from peripheral blood of cancer patients, we screened T-EV in 
vitro for the expression of different tumor markers which allow the discrimination of T-EV from non-malignant EV. EpCAM was identified as the only protein which was present on TMV as well as T-Exo. In line with our in vitro data, EpCAM has also been identified by other authors in vivo on EV isolated from ascites and serum of ovarian cancer patients (Keller et al, 2009). These findings argued for the possible use of EpCAM as marker for the detection of circulating T-EV. Based on the study from Taylor et al who described an EpCAM-based MACS approach to specifically sort T-Exo from serum samples $(2,5 \mathrm{ml})$ of ovarian cancer patients (Taylor \& Gercel-Taylor, 2008), we tried to adopt this method for the specific isolation of T-MV as well as T-Exo from serum samples of metastatic cancer patients.

First, the method was tested in vitro by spiking human AB serum samples with T-MV or TExo. Even though we optimized the concentration of anti-EpCAM MicroBeads as well as the type of separation column, this method allowed us to sort only $20 \%$ of the input T-MV in vitro. Furthermore, we were not able to sort T-Exo from $\mathrm{AB}$ serum, possibly due to the low expression of EpCAM on T-Exo. Nonetheless, we decided to test whether the established method would work in vivo, at least for MV. Since in the study from Taylor et al a positive correlation was found between the number of EpCAM-positive Exo and tumor stage (Taylor \& Gercel-Taylor, 2008), we chose cancer patients with metastatic disease who should have the highest number of circulating T-EV in their blood. Although we were indeed able to isolate high amounts of EpCAM-positive MV from some patient samples, further characterization of the sorted vesicles revealed that most of the sorted vesicles were falsepositively selected MV from normal blood cells which in some cases even lacked EpCAMexpression. This control procedure had not been performed in the other two studies (Keller et al, 2009; Taylor \& Gercel-Taylor, 2008). Thus, in our hands, EpCAM-based MACS does not seem to specifically capture T-EV from human serum samples which might be explained by the low number of EpCAM-positive MV present even in advanced cancer patients. However, it cannot be excluded that MACS might work with MicroBeads coated with antibodies directed against other tumor proteins which are specific for T-EV and are expressed at higher levels on the surface of the vesicles.

\subsubsection{Detection of circulating T-MV}

Since it was not possible to specifically sort T-EV from human blood samples, we isolated the complete EV population from peripheral blood of metastatic cancer patients. Based on the previously identified markers for $\mathrm{T}-\mathrm{MV}$ as well as non-malignant $\mathrm{MV}$ in our in vitro screenings, we analyzed which MV populations are present in blood and whether we can 
identify circulating T-MV without any previous sorting step. Since so far we could not identify tumor-specific Exo markers, except EpCAM, we choose to not perform further characterization of patient-derived Exo populations.

Complete MV and Exo could be successfully isolated from serum samples of EDTAanticoagulated blood from cancer and control patients. Plasma from lithium-heparinanticoagulated blood was less suited for EV isolation since it displayed delayed agglutination which corresponded with significantly reduced MV counts. In particular, the number of CD62P- and EMMPRIN-positive MV was reduced which we had identified as markers for PMV. These observations seem to confirm recent data which suggest that heparin interacts with certain MV and forms immune complexes with platelets (Krauel et al, 2012; Maguire et al, 2012).

Several studies report an increase in the total number of isolated EV from cancer patients (Baran et al, 2010; Ghosh et al, 2010), while others only find an increase in protein density, but not in the amount of EV (Peinado et al, 2012). Elevated EV counts are not only associated with cancer, but are also attributed to cardiovascular diseases including hypertension, diabetes or arteriosclerosis which mainly occur in elderly patients (VanWijk et al, 2003). Although the patients included in our preliminary study were slightly older compared to the control group, we did not detect a significant increase in the total number of isolated $\mathrm{MV}$, whereas Exo were slightly, but not significantly, elevated. A larger group of patients with age-matched controls will be necessary to further strengthen this finding. Interestingly, while most blood-derived MV markers did not differ between patients and controls, we observed an increase in the number of leukocyte-derived CD45-positive MV in cancer patients which has already been described before in breast cancer patients with advanced disease (Toth et al, 2008b). However, it still has to be clarified whether this is caused by an influence on leukocyte cell counts or on MV release by leukocytes. While some studies of healthy individuals or breast cancer patients reported that more than $80 \%$ of MV in blood are derived from platelets (Flaumenhaft et al, 2009; Toth et al, 2008a), we observed expression of the platelet marker CD62P on only around 30-35\% of the isolated MV (see Fig. 42). However, P-MV still represented the largest group of circulating blood-derived MV in the samples.

Next to the analysis of benign MV populations in blood, we aimed to identify circulating TMV in cancer patients based on the established tumor markers EGFR, MUC1, EpCAM and EMMPRIN. We detected expression of the tumor marker EGFR on MV from 2 out of 18 investigated cancer patients. Although EGFR overexpression is mostly attributed to colorectal 
cancer, it has also been associated with breast, lung or gastric cancer (Spano et al, 2005). Correspondingly, one patient with MV-bound EGFR suffered from primary breast cancer, the other from primary lung cancer. In breast cancer patients, EGFR overexpression is mostly associated with the triple-negative subtype (Burness et al, 2010) which corresponds to the data from our in vitro screening of T-MV, where EGFR was only found on T-MV from the MDA-MB231 triple-negative breast cancer cell line (see Fig. 32). Apart from breast cancer, first clinical investigations detected Exo-bound EGFRvIII in 7 out of 25 investigated glioblastoma patients by nested RT-PCR, whereas it was present in none of the 30 control patients (Skog et al, 2008).

Besides EGFR, we identified MUC1 on MV from 5 (lung cancer: 3, breast cancer: 1, colon cancer: 1) out of 19 cancer patients. While in healthy individuals MUC1 is present on specialized secretory epithelia as well as in epithelia lining the respiratory and gastrointestinal tract (Kufe, 2009), it was not detectable on MV from peripheral blood of any of the investigated controls suggesting its specificity for T-MV. MUC1, corresponding to the serum marker CA 15-3, is specifically found at high levels in various epithelial cancers (Hollingsworth \& Swanson, 2004; Szabo, 2003). Moreover, it is used as a prognostic parameter in breast cancer (Duffy et al, 2000) and was proposed to contribute to tumor invasion and metastasis (Hollingsworth \& Swanson, 2004).

In contrast to EGFR and MUC1, EpCAM was also found on MV of some of the control patients. However, the EpCAM-levels were significantly increased in MV derived from cancer patients, although the number of EpCAM-positive MV was still comparably low. The low number of EpCAM-positive MV in cancer patients might explain the failure of EpCAMbased sorting of T-MV as discussed above. One disadvantage of EpCAM as a tumor marker is that it is only expressed on cancers of epithelial origin and cannot be utilized for malignancies of mesenchymal provenience, such as sarcomas. EMMPRIN, in contrast, is frequently overexpressed in many types of malignant tumors including lymphomas, sarcomas and carcinomas ( $\mathrm{Li}$ et al, 2009; Nabeshima et al, 2004; Riethdorf et al, 2006). In line with this, we found EMMPRIN-positive MV in the blood of all investigated cancer patients (21/21). Although EMMPRIN was also detected on MV from control patients (12/13), the number of EMMPRIN-positive MV was highly increased in metastatic cancer patients. This finding retrospectively reinforces a study from Baran et al who detected EMMPRIN in a small series of plasma samples of gastric cancer patients. While no direct EMMPRIN staining was performed in that work, centrifugation of the samples at $50.000 \mathrm{~g}$ resulted in a loss of EMMPRIN expression suggesting that it was associated with MV (Baran et al, 2010). 
Since EMMPRIN is also expressed on MV shed by benign blood cells like e.g. P-MV or M $\phi-$ MV, we performed double stainings with the tumor marker MUC1 to confirm that the EMMPRIN-positive MV are indeed released by tumor cells. From the investigated breast cancer patients two were positive for MUC1 and both showed a co-localization of MUC1 and EMMPRIN on their MV which was not seen on MV from control patients. This confirms that parts of the EMMPRIN-positive MV are indeed T-MV and circulate at high levels in the blood of metastatic cancer patients suggesting that they could be used as potential biomarkers. However, it still remains to be investigated whether the level of EMMPRIN-positive MV correlates with prognosis or disease progression to evaluate its usability as potential biomarker. Moreover, considering their effect on tumor invasion in vitro, it is possible that EMMPRIN-positive MV are not only important for prognosis or diagnosis, but also contribute to tumor progression in vivo. In this context, further studies are needed to address this question. 


\section{Summary and conclusions}

This study investigated the role of EV derived from human breast cancer cells for tumor invasion, which represents a crucial step in cancer progression and metastasis formation. EV are continuously released from every cell in the body, although increased shedding can be observed during cellular activation. However, functional studies investigating their biological impact are limited due to a lack of suitable markers to discriminate between MV and Exo. Using a differential ultracentrifugation approach, we were able to separately isolate and analyze MV and Exo. Surprisingly, even though both populations differ in their biogenesis and protein expression pattern, they were found to exert similar biological effects: 1) MV and Exo increase breast cancer cell invasion in an autologous feedback loop; 2) MV and Exo are able to reprogram $\mathrm{M} \phi$ into a tumor-supporting phenotype by inducing Wnt5a.

Although some studies have already demonstrated the pro-invasive potential of T-Exo, the influence of T-MV on tumor invasion has not been investigated yet. In this study, we showed that T-MV, comparable to T-Exo, influence malignant invasion i) by acting back on the tumor cells themselves and ii) by mediating the pro-invasive communication between tumor cells and $\mathrm{M} \phi$. The first route is exemplified in Fig. 45: T-MV strongly increased the invasive potential of surrounding tumor cells in an autologous and heterologous loop. This effect was not observed when tumor cells were stimulated with the corresponding particle-free supernatant or with benign MV. We showed that uptake of MV into their recipient cell in a dynamin-dependent way as well as expression of tumor-supporting proteins on T-MV are prerequisites for MV function. EMMPRIN is one of these proteins and was identified as a sensitive and specific marker for MV.

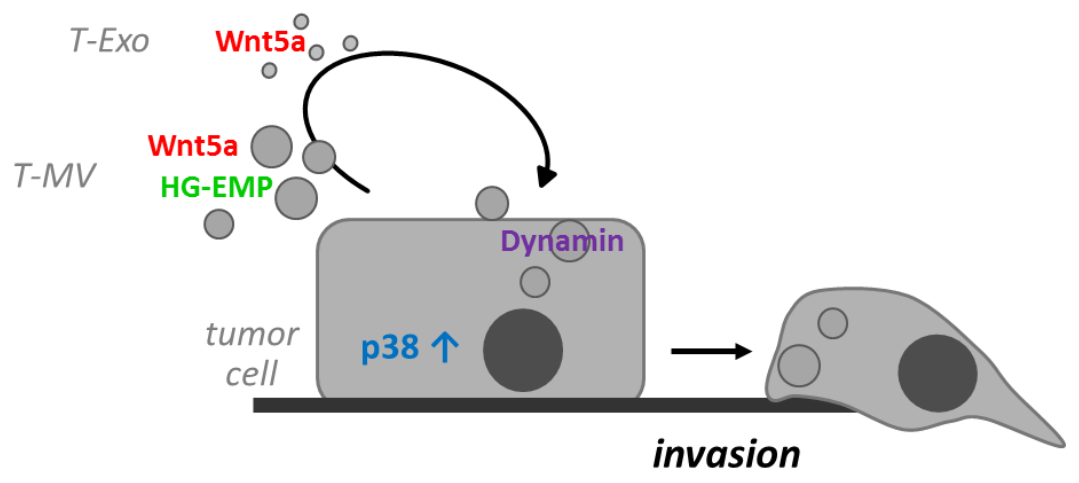

Fig. 45: Schematic presentation of EV-mediated tumor-tumor crosstalk in human breast cancer

Breast cancer cells release T-EV which are enriched in pro-invasive factors (e.g. HG-EMMPRIN or Wnt5a). These T-EV act back on the surrounding tumor cells where they can either horizontally transfer their messages after dynamin-dependent incorporation or they interact with cell surface-associated receptors. T-MV stimulation activates p38/MAPK signaling which is partly involved in the subsequent increase in tumor invasion. 
All pro-invasive T-MV carried a highly glycosylated form of EMMPRIN which was found to be N-glycosylated at N160 and N268. Knockdown of EMMPRIN or deglycosylation partly reduced the invasive potential of T-MV confirming that HG-EMMPRIN is one of the proinvasive factors on T-MV. The MV-induced tumor invasion was not mediated by MMP induction, but by activation of the EMMPRIN downstream target p38/MAPK.

The second route is shown in Fig. 46: T-EV from human breast cancer cells, apart from influencing the surrounding tumor cells, were found to reprogram $\mathrm{M} \phi$ by inducing expression of the non-canonical Wnt ligand Wnt5a. Wnt5a expression is associated with a tolerogenic phenotype in $\mathrm{M} \phi$ (Bergenfelz et al, 2012) and was identified earlier as indispensable for the pro-invasive tumor-M $\phi$ crosstalk by our group (Pukrop et al, 2006). In case of T-MV, induction of Wnt5a is independent of MV uptake and is mediated by p38/MAPK signaling which can be antagonized by the Wnt-inhibitor DKK-1. These results suggest a receptorligand-mediated interaction at the M $\phi$ surface. After T-EV stimulation, M $\phi$ shuttle the produced Wnt5a protein out of the cells on their own EV, a process which depends on the expression of the seven-pass transmembrane protein Evi. Wnt5a is delivered to the tumor cells where it is incorporated and supports tumor progression. EV-bound Wnt5a is not only involved in pro-invasive tumor-M $\phi$ crosstalk, but was also shown to be released on T-EV from Wnt5a-positive breast cancer cell lines (e.g. SK-BR-3) and to contribute to their proinvasive effect on neighboring tumor cells (Fig. 45). Thus, it seems highly likely that both routes, the tumor-tumor as well as the tumor-M $\phi$ loop are interconnected and act synergistically in vivo.

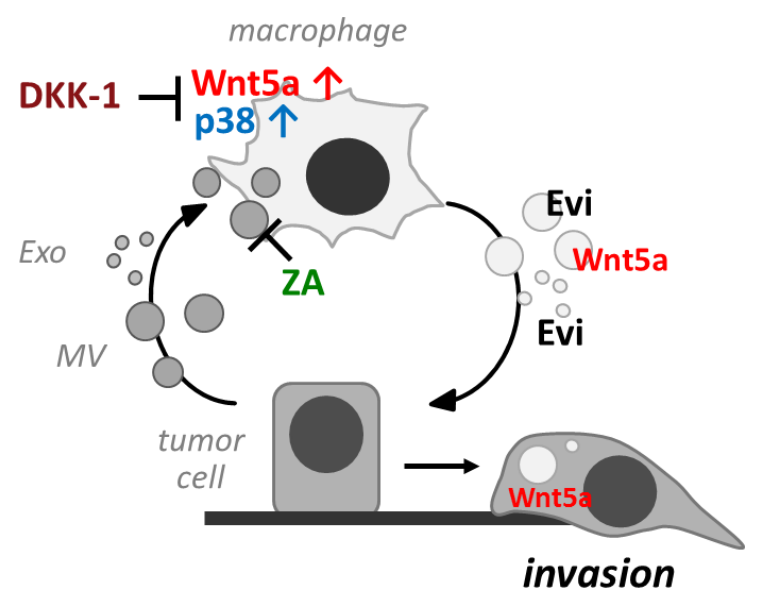

Fig. 46: Schematic presentation of $\mathrm{EV}$-mediated tumor-M $\phi$ crosstalk in human breast cancer

On the one hand, T-EV released by human breast cancer cells influence human $M \phi$ by horizontally transferring tumor-supporting proteins or nucleic acids which need to be incorporated to fulfill their function. MV uptake can be impaired by the bisphosphonate ZA. On the other hand, T-EV interact with an unknown cell surface-bound receptor to induce expression of Wnt5a which is mediated by p38 signaling and can be antagonized by DKK-1. Stimulated $\mathrm{M} \phi$ then export the produced Wnt5a protein in an Evi-dependent way on their own EV and shuttle it to the tumor cells where it is incorporated and increases tumor invasion. Modified from (Menck et al, 2013). 
In order to further characterize the situation in vivo, we established a protocol for the isolation of the complete EV populations from peripheral blood of cancer patients and controls. While the absolute amount of EV did not differ between cancer patients and controls, we identified circulating $\mathrm{MUC1}^{+} / \mathrm{EMMPRIN}^{+} \mathrm{T}-\mathrm{MV}$ at high numbers in the blood of metastatic breast cancer patients suggesting their potential use as novel biomarkers in cancer.

Taken together, in this study we demonstrate that human breast cancer cells release T-EV into the local tumor microenvironment as well as the bloodstream. These T-EV represent novel means of intercellular communication which the tumor cells use to influence the adjacent stroma and tumor cells to create a favorable and permissive tumor niche and drive progression to metastasis. 


\section{Bibliography}

Ai X, Do AT, Lozynska O, Kusche-Gullberg M, Lindahl U, Emerson CP, Jr. (2003) QSulf1 remodels the 6-O sulfation states of cell surface heparan sulfate proteoglycans to promote Wnt signaling. J Cell Biol 162: 341-351

Al-Nedawi K, Meehan B, Micallef J, Lhotak V, May L, Guha A, Rak J (2008) Intercellular transfer of the oncogenic receptor EGFRvIII by microvesicles derived from tumour cells. Nat Cell Biol 10: $619-624$

Alix-Panabieres C, Brouillet JP, Fabbro M, Yssel H, Rousset T, Maudelonde T, Choquet-Kastylevsky G, Vendrell JP (2005) Characterization and enumeration of cells secreting tumor markers in the peripheral blood of breast cancer patients. J Immunol Methods 299: 177-188

Allavena P, Sica A, Solinas G, Porta C, Mantovani A (2008) The inflammatory micro-environment in tumor progression: the role of tumor-associated macrophages. Critical reviews in oncology/hematology 66: 1-9

Angelucci A, D'Ascenzo S, Festuccia C, Gravina GL, Bologna M, Dolo V, Pavan A (2000) Vesicleassociated urokinase plasminogen activator promotes invasion in prostate cancer cell lines. Clin Exp Metastasis 18: 163-170

Aonuma M, Saeki Y, Akimoto T, Nakayama Y, Hattori C, Yoshitake Y, Nishikawa K, Shibuya M, Tanaka NG (1999) Vascular endothelial growth factor overproduced by tumour cells acts predominantly as a potent angiogenic factor contributing to malignant progression. International journal of experimental pathology 80: 271-281

Aung T, Chapuy B, Vogel D, Wenzel D, Oppermann M, Lahmann M, Weinhage T, Menck K, Hupfeld T, Koch R, Trumper L, Wulf GG (2011) Exosomal evasion of humoral immunotherapy in aggressive B-cell lymphoma modulated by ATP-binding cassette transporter A3. P Natl Acad Sci USA 108: 15336-15341

Baj-Krzyworzeka M, Szatanek R, Weglarczyk K, Baran J, Urbanowicz B, Branski P, Ratajczak MZ, Zembala M (2006) Tumour-derived microvesicles carry several surface determinants and mRNA of tumour cells and transfer some of these determinants to monocytes. Cancer Immunol Immun 55: 808-818

Banziger C, Soldini D, Schutt C, Zipperlen P, Hausmann G, Basler K (2006) Wntless, a conserved membrane protein dedicated to the secretion of Wnt proteins from signaling cells. Cell 125: 509-522

Baran J, Baj-Krzyworzeka M, Weglarczyk K, Szatanek R, Zembala M, Barbasz J, Czupryna A, Szczepanik A, Zembala M (2010) Circulating tumour-derived microvesicles in plasma of gastric cancer patients. Cancer Immunol Immun 59: 841-850

Bartscherer K, Boutros M (2008) Regulation of Wnt protein secretion and its role in gradient formation. EMBO reports 9: 977-982

Bartscherer K, Pelte N, Ingelfinger D, Boutros M (2006) Secretion of Wnt Ligands requires Evi, a conserved transmembrane protein. Cell 125: 523-533

Bergenfelz C, Medrek C, Ekstrom E, Jirstrom K, Janols H, Wullt M, Bredberg A, Leandersson K (2012) Wnt5a induces a tolerogenic phenotype of macrophages in sepsis and breast cancer patients. J Immunol 188: 5448-5458

Bergsmedh A, Szeles A, Henriksson M, Bratt A, Folkman MJ, Spetz AL, Holmgren L (2001) Horizontal transfer of oncogenes by uptake of apoptotic bodies. Proc Natl Acad Sci U S A 98: 6407-6411

Berridge MV, Tan A, McCoy KD, Wang R (1996) The Biochemical and Cellular Basis of Cell Proliferation Assays That Use Tetrazolium Salts. Biochemica 4: 14-19 
Bevers EM, Comfurius P, Dekkers DW, Zwaal RF (1999) Lipid translocation across the plasma membrane of mammalian cells. Biochimica et biophysica acta 1439: 317-330

Bianco F, Pravettoni E, Colombo A, Schenk U, Moller T, Matteoli M, Verderio C (2005) Astrocytederived ATP induces vesicle shedding and IL-1 beta release from microglia. J Immunol 174: 7268-7277

Bissell MJ, Radisky D (2001) Putting tumours in context. Nature reviews Cancer 1: 46-54

Biswas C, Zhang Y, DeCastro R, Guo H, Nakamura T, Kataoka H, Nabeshima K (1995) The human tumor cell-derived collagenase stimulatory factor (renamed EMMPRIN) is a member of the immunoglobulin superfamily. Cancer Res 55: 434-439

Blanc E, Goldschneider D, Douc-Rasy S, Benard J, Raguenez G (2005) Wnt-5a gene expression in malignant human neuroblasts. Cancer Lett 228: 117-123

Blanchard N, Lankar D, Faure F, Regnault A, Dumont C, Raposo G, Hivroz C (2002) TCR activation of human $\mathrm{T}$ cells induces the production of exosomes bearing the TCR/CD3/zeta complex. $J$ Immunol 168: 3235-3241

Blumenthal A, Ehlers S, Lauber J, Buer J, Lange C, Goldmann T, Heine H, Brandt E, Reiling N (2006) The Wingless homolog, WNT5A and its receptor Frizzled-5 regulate inflammatory responses of human mononuclear cells induced by microbial stimulation. Blood 108: 965-973

Bo H, Zhang S, Gao L, Chen Y, Zhang J, Chang X, Zhu M (2013) Upregulation of Wnt5a promotes epithelial-to-mesenchymal transition and metastasis of pancreatic cancer cells. BMC cancer 13: 496

Bougatef F, Quemener C, Kellouche S, Naimi B, Podgorniak MP, Millot G, Gabison EE, Calvo F, Dosquet C, Lebbe C, Menashi S, Mourah S (2009) EMMPRIN promotes angiogenesis through hypoxia-inducible factor-2alpha-mediated regulation of soluble VEGF isoforms and their receptor VEGFR-2. Blood 114: 5547-5556

Buechling T, Chaudhary V, Spirohn K, Weiss M, Boutros M (2011) p24 proteins are required for secretion of Wnt ligands. EMBO reports 12: 1265-1272

Bui TD, Zhang L, Rees MC, Bicknell R, Harris AL (1997) Expression and hormone regulation of Wnt2, 3, 4, 5a, 7a, 7b and 10b in normal human endometrium and endometrial carcinoma. British journal of cancer 75: 1131-1136

Burness ML, Grushko TA, Olopade OI (2010) Epidermal growth factor receptor in triple-negative and basal-like breast cancer: promising clinical target or only a marker? Cancer journal 16: 23-32

Butler TP, Gullino PM (1975) Quantitation of cell shedding into efferent blood of mammary adenocarcinoma. Cancer Res 35: 512-516

Cailleau R, Young R, Olive M, Reeves WJ (1974) Breast Tumor-Cell Lines from Pleural Effusions. J Natl Cancer I 53: 661-674

Canfran-Duque A, Pastor O, Quintana-Portillo R, Lerma M, de la Pena G, Martin-Hidalgo A, Fernandez-Hernando C, Lasuncion MA, Busto R (2013) Curcumin promotes exosomes/microvesicles secretion that attenuates lysosomal cholesterol traffic impairment. Molecular nutrition \& food research

Castellana D, Zobairi F, Martinez MC, Panaro MA, Mitolo V, Freyssinet JM, Kunzelmann C (2009) Membrane Microvesicles as Actors in the Establishment of a Favorable Prostatic Tumoral Niche: A Role for Activated Fibroblasts and CX3CL1-CX3CR1 Axis. Cancer Res 69: 785793

Ching W, Nusse R (2006) A dedicated Wnt secretion factor. Cell 125: 432-433

Cocucci E, Racchetti G, Meldolesi J (2009) Shedding microvesicles: artefacts no more. Trends Cell Biol 19: 43-51 
Cruz-Munoz W, Khokha R (2008) The role of tissue inhibitors of metalloproteinases in tumorigenesis and metastasis. Critical reviews in clinical laboratory sciences 45: 291-338

Curtin KD, Meinertzhagen IA, Wyman RJ (2005) Basigin (EMMPRIN/CD147) interacts with integrin to affect cellular architecture. J Cell Sci 118: 2649-2660

D'Souza-Schorey C, Clancy JW (2012) Tumor-derived microvesicles: shedding light on novel microenvironment modulators and prospective cancer biomarkers. Genes \& development 26: 1287-1299

Dashevsky O, Varon D, Brill A (2009) Platelet-derived microparticles promote invasiveness of prostate cancer cells via upregulation of MMP-2 production. Int J Cancer 124: 1773-1777

Dawood S, Broglio K, Buzdar AU, Hortobagyi GN, Giordano SH (2010) Prognosis of women with metastatic breast cancer by HER2 status and trastuzumab treatment: an institutional-based review. Journal of clinical oncology : official journal of the American Society of Clinical Oncology 28: 92-98

De A (2011) Wnt/Ca2+ signaling pathway: a brief overview. Acta biochimica et biophysica Sinica 43: 745-756

de Gassart A, Geminard C, Fevrier B, Raposo G, Vidal M (2003) Lipid raft-associated protein sorting in exosomes. Blood 102: 4336-4344

Decaussin M, Sartelet H, Robert C, Moro D, Claraz C, Brambilla C, Brambilla E (1999) Expression of vascular endothelial growth factor (VEGF) and its two receptors (VEGF-R1-Flt1 and VEGF$\mathrm{R} 2-\mathrm{Flk1/KDR}$ ) in non-small cell lung carcinomas (NSCLCs): correlation with angiogenesis and survival. The Journal of pathology 188: 369-377

Dejmek J, Dejmek A, Safholm A, Sjolander A, Andersson T (2005) Wnt-5a protein expression in primary dukes B colon cancers identifies a subgroup of patients with good prognosis. Cancer Res 65: $9142-9146$

Del Conde I, Shrimpton CN, Thiagarajan P, Lopez JA (2005) Tissue-factor-bearing microvesicles arise from lipid rafts and fuse with activated platelets to initiate coagulation. Blood 106: 16041611

Dignat-George F, Boulanger CM (2011) The many faces of endothelial microparticles. Arteriosclerosis, thrombosis, and vascular biology 31: 27-33

Dissanayake SK, Wade M, Johnson CE, O'Connell MP, Leotlela PD, French AD, Shah KV, Hewitt KJ, Rosenthal DT, Indig FE, Jiang Y, Nickoloff BJ, Taub DD, Trent JM, Moon RT, Bittner M, Weeraratna AT (2007) The Wnt5A/protein kinase C pathway mediates motility in melanoma cells via the inhibition of metastasis suppressors and initiation of an epithelial to mesenchymal transition. The Journal of biological chemistry 282: 17259-17271

Doherty GJ, McMahon HT (2009) Mechanisms of endocytosis. Annual review of biochemistry 78: 857-902

Dolo V, D'Ascenzo S, Violini S, Pompucci L, Festuccia C, Ginestra A, Vittorelli ML, Canevari S, Pavan A (1999) Matrix-degrading proteinases are shed in membrane vesicles by ovarian cancer cells in vivo and in vitro. Clin Exp Metastasis 17: 131-140

Dolo V, Ginestra A, Cassara D, Violini S, Lucania G, Torrisi MR, Nagase H, Canevari S, Pavan A, Vittorelli ML (1998) Selective localization of matrix metalloproteinase 9, beta1 integrins, and human lymphocyte antigen class I molecules on membrane vesicles shed by 8701 -BC breast carcinoma cells. Cancer Res 58: 4468-4474

Duffy MJ, Shering S, Sherry F, McDermott E, O'Higgins N (2000) CA 15-3: a prognostic marker in breast cancer. The International journal of biological markers 15: 330-333

Dunnwald LK, Rossing MA, Li CI (2007) Hormone receptor status, tumor characteristics, and prognosis: a prospective cohort of breast cancer patients. Breast cancer research : BCR 9: R6 
Dvorak HF (1986) Tumors: wounds that do not heal. Similarities between tumor stroma generation and wound healing. The New England journal of medicine 315: 1650-1659

Eagle H (1955) The minimum vitamin requirements of the L and HeLa cells in tissue culture, the production of specific vitamin deficiencies, and their cure. The Journal of experimental medicine 102: 595-600

Egeblad M, Werb Z (2002) New functions for the matrix metalloproteinases in cancer progression. Nature reviews Cancer 2: 161-174

Elmore S (2007) Apoptosis: a review of programmed cell death. Toxicologic pathology 35: 495-516

Escrevente C, Keller S, Altevogt P, Costa J (2011) Interaction and uptake of exosomes by ovarian cancer cells. BMC cancer 11: 108

Fadok VA, Voelker DR, Campbell PA, Cohen JJ, Bratton DL, Henson PM (1992) Exposure of phosphatidylserine on the surface of apoptotic lymphocytes triggers specific recognition and removal by macrophages. J Immunol 148: 2207-2216

Fevrier B, Raposo G (2004) Exosomes: endosomal-derived vesicles shipping extracellular messages. Current opinion in cell biology 16: 415-421

Fidler IJ (1970) Metastasis: guantitative analysis of distribution and fate of tumor embolilabeled with 125 I-5-iodo-2'-deoxyuridine. J Natl Cancer Inst 45: 773-782

Flaumenhaft R, Dilks JR, Richardson J, Alden E, Patel-Hett SR, Battinelli E, Klement GL, SolaVisner M, Italiano JE, Jr. (2009) Megakaryocyte-derived microparticles: direct visualization and distinction from platelet-derived microparticles. Blood 113: 1112-1121

Fox SB, Smith K, Hollyer J, Greenall M, Hastrich D, Harris AL (1994) The epidermal growth factor receptor as a prognostic marker: results of 370 patients and review of 3009 patients. Breast cancer research and treatment 29: 41-49

Frantz C, Stewart KM, Weaver VM (2010) The extracellular matrix at a glance. J Cell Sci 123: 41954200

Gasser O, Hess C, Miot S, Deon C, Sanchez JC, Schifferli JA (2003) Characterisation and properties of ectosomes released by human polymorphonuclear neutrophils. Experimental cell research 285: $243-257$

Ghosh AK, Secreto CR, Knox TR, Ding W, Mukhopadhyay D, Kay NE (2010) Circulating microvesicles in B-cell chronic lymphocytic leukemia can stimulate marrow stromal cells: implications for disease progression. Blood 115: 1755-1764

Gilbey AM, Burnett D, Coleman RE, Holen I (2004) The detection of circulating breast cancer cells in blood. Journal of clinical pathology 57: 903-911

Ginestra A, Monea S, Seghezzi G, Dolo V, Nagase H, Mignatti P, Vittorelli ML (1997) Urokinase plasminogen activator and gelatinases are associated with membrane vesicles shed by human HT1080 fibrosarcoma cells. The Journal of biological chemistry 272: 17216-17222

Gnant M (2009) Bisphosphonates in the prevention of disease recurrence: current results and ongoing trials. Current cancer drug targets 9: 824-833

Gnant M (2011) Zoledronic acid in breast cancer: latest findings and interpretations. Therapeutic advances in medical oncology 3: 293-301

Golpon HA, Fadok VA, Taraseviciene-Stewart L, Scerbavicius R, Sauer C, Welte T, Henson PM, Voelkel NF (2004) Life after corpse engulfment: phagocytosis of apoptotic cells leads to VEGF secretion and cell growth. Faseb J 18: 1716-1718

Gordon MD, Nusse R (2006) Wnt signaling: multiple pathways, multiple receptors, and multiple transcription factors. The Journal of biological chemistry 281: 22429-22433

Gordon S (2003) Alternative activation of macrophages. Nat Rev Immunol 3: 23-35 
Goswami S, Sahai E, Wyckoff JB, Cammer M, Cox D, Pixley FJ, Stanley ER, Segall JE, Condeelis JS (2005) Macrophages promote the invasion of breast carcinoma cells via a colony-stimulating factor-1/epidermal growth factor paracrine loop. Cancer Res 65: 5278-5283

Graves LE, Ariztia EV, Navari JR, Matzel HJ, Stack MS, Fishman DA (2004) Proinvasive properties of ovarian cancer ascites-derived membrane vesicles. Cancer Res 64: 7045-7049

Greco V, Hannus M, Eaton S (2001) Argosomes: a potential vehicle for the spread of morphogens through epithelia. Cell 106: 633-645

Gross JC, Chaudhary V, Bartscherer K, Boutros M (2012) Active Wnt proteins are secreted on exosomes. Nat Cell Biol 14: 1036-+

Gruenberg J, Stenmark H (2004) The biogenesis of multivesicular endosomes. Nature reviews Molecular cell biology 5: 317-323

Guo H, Zucker S, Gordon MK, Toole BP, Biswas C (1997) Stimulation of matrix metalloproteinase production by recombinant extracellular matrix metalloproteinase inducer from transfected Chinese hamster ovary cells. The Journal of biological chemistry 272: 24-27

Gyorgy B, Modos K, Pallinger E, Paloczi K, Pasztoi M, Misjak P, Deli MA, Sipos A, Szalai A, Voszka I, Polgar A, Toth K, Csete M, Nagy G, Gay S, Falus A, Kittel A, Buzas EI (2011a) Detection and isolation of cell-derived microparticles are compromised by protein complexes resulting from shared biophysical parameters. Blood 117: e39-48

Gyorgy B, Szabo TG, Pasztoi M, Pal Z, Misjak P, Aradi B, Laszlo V, Pallinger E, Pap E, Kittel A, Nagy G, Falus A, Buzas EI (2011b) Membrane vesicles, current state-of-the-art: emerging role of extracellular vesicles. Cell Mol Life Sci 68: 2667-2688

Hafner M, Orosz P, Kruger A, Mannel DN (1996) TNF promotes metastasis by impairing natural killer cell activity. Int J Cancer 66: 388-392

Hagemann T, Robinson SC, Schulz M, Trumper L, Balkwill FR, Binder C (2004) Enhanced invasiveness of breast cancer cell lines upon co-cultivation with macrophages is due to TNFalpha dependent up-regulation of matrix metalloproteases. Carcinogenesis 25: 1543-1549

Hagemann T, Wilson J, Kulbe H, Li NF, Leinster DA, Charles K, Klemm F, Pukrop T, Binder C, Balkwill FR (2005) Macrophages induce invasiveness of epithelial cancer cells via NF-kappa B and JNK. J Immunol 175: 1197-1205

Halicka HD, Bedner E, Darzynkiewicz Z (2000) Segregation of RNA and separate packaging of DNA and RNA in apoptotic bodies during apoptosis. Experimental cell research 260: 248-256

Han YH, Moon HJ, You BR, Park WH (2009) The effect of MG132, a proteasome inhibitor on HeLa cells in relation to cell growth, reactive oxygen species and GSH. Oncology reports 22: 215221

Hanahan D, Weinberg RA (2000) The hallmarks of cancer. Cell 100: 57-70

Hanaki H, Yamamoto H, Sakane H, Matsumoto S, Ohdan H, Sato A, Kikuchi A (2012) An anti-Wnt5a antibody suppresses metastasis of gastric cancer cells in vivo by inhibiting receptor-mediated endocytosis. Molecular cancer therapeutics 11: 298-307

Hardin J, King RS (2008) The long and the short of Wnt signaling in C. elegans. Current opinion in genetics \& development 18: $362-367$

Harding C, Heuser J, Stahl P (1983) Receptor-mediated endocytosis of transferrin and recycling of the transferrin receptor in rat reticulocytes. J Cell Biol 97: 329-339

Helley D, Banu E, Bouziane A, Banu A, Scotte F, Fischer AM, Oudard S (2009) Platelet microparticles: a potential predictive factor of survival in hormone-refractory prostate cancer patients treated with docetaxel-based chemotherapy. European urology 56: 479-484 
Hemler ME (2003) Tetraspanin proteins mediate cellular penetration, invasion, and fusion events and define a novel type of membrane microdomain. Annual review of cell and developmental biology 19: 397-422

Henson PM, Bratton DL, Fadok VA (2001) Apoptotic cell removal. Curr Biol 11: R795-805

Higginbotham JN, Demory Beckler M, Gephart JD, Franklin JL, Bogatcheva G, Kremers GJ, Piston DW, Ayers GD, McConnell RE, Tyska MJ, Coffey RJ (2011) Amphiregulin exosomes increase cancer cell invasion. Curr Biol 21: 779-786

Higgins MJ, Stearns V (2009) Understanding resistance to tamoxifen in hormone receptor-positive breast cancer. Clinical chemistry 55: 1453-1455

Hilakivi-Clarke L (2000) Estrogens, BRCA1, and breast cancer. Cancer Res 60: 4993-5001

Hiraga T, Williams PJ, Ueda A, Tamura D, Yoneda T (2004) Zoledronic acid inhibits visceral metastases in the 4T1/luc mouse breast cancer model. Clinical cancer research : an official journal of the American Association for Cancer Research 10: 4559-4567

Hiraoka K, Zenmyo M, Watari K, Iguchi H, Fotovati A, Kimura YN, Hosoi F, Shoda T, Nagata K, Osada H, Ono M, Kuwano M (2008) Inhibition of bone and muscle metastases of lung cancer cells by a decrease in the number of monocytes/macrophages. Cancer science 99: 1595-1602

Hollingsworth MA, Swanson BJ (2004) Mucins in cancer: protection and control of the cell surface. Nature reviews Cancer 4: 45-60

Holme PA, Orvim U, Hamers MJ, Solum NO, Brosstad FR, Barstad RM, Sakariassen KS (1997) Shear-induced platelet activation and platelet microparticle formation at blood flow conditions as in arteries with a severe stenosis. Arteriosclerosis, thrombosis, and vascular biology 17: 646-653

Hood JL, San RS, Wickline SA (2011) Exosomes released by melanoma cells prepare sentinel lymph nodes for tumor metastasis. Cancer Res 71: 3792-3801

Hristov M, Erl W, Linder S, Weber PC (2004) Apoptotic bodies from endothelial cells enhance the number and initiate the differentiation of human endothelial progenitor cells in vitro. Blood 104: $2761-2766$

Hsu C, Morohashi Y, Yoshimura S, Manrique-Hoyos N, Jung S, Lauterbach MA, Bakhti M, Gronborg M, Mobius W, Rhee J, Barr FA, Simons M (2010) Regulation of exosome secretion by Rab35 and its GTPase-activating proteins TBC1D10A-C. J Cell Biol 189: 223-232

Hugel B, Carmen M, Martinez MC, Kunzelmann C, Freyssinet JM (2005) Membrane microparticles: Two sides of the coin. Physiology 20: 22-27

Hutchinson L (2010) Breast cancer: challenges, controversies, breakthroughs. Nature reviews Clinical oncology 7: 669-670

Janda CY, Waghray D, Levin AM, Thomas C, Garcia KC (2012) Structural basis of Wnt recognition by Frizzled. Science 337: 59-64

Janowska-Wieczorek A, Wysoczynski M, Kijowski J, Marquez-Curtis L, Machalinski B, Ratajczak J, Ratajczak MZ (2005) Microvesicles derived from activated platelets induce metastasis and angiogenesis in lung cancer. Int J Cancer 113: 752-760

Jayachandran M, Miller VM, Heit JA, Owen WG (2012) Methodology for isolation, identification and characterization of microvesicles in peripheral blood. J Immunol Methods 375: 207-214

Jiang JL, Zhou Q, Yu MK, Ho LS, Chen ZN, Chan HC (2001) The involvement of HAb18G/CD147 in regulation of store-operated calcium entry and metastasis of human hepatoma cells. The Journal of biological chemistry 276: 46870-46877

Jiang W, Crossman DK, Mitchell EH, Sohn P, Crowley MR, Serra R (2013) WNT5A inhibits metastasis and alters splicing of Cd44 in breast cancer cells. Plos One 8: e58329 
Joyce JA, Pollard JW (2009) Microenvironmental regulation of metastasis. Nature reviews Cancer 9: 239-252

Kadowaki T, Wilder E, Klingensmith J, Zachary K, Perrimon N (1996) The segment polarity gene porcupine encodes a putative multitransmembrane protein involved in Wingless processing. Genes \& development 10: 3116-3128

Kataoka H, DeCastro R, Zucker S, Biswas C (1993) Tumor cell-derived collagenase-stimulatory factor increases expression of interstitial collagenase, stromelysin, and 72-kDa gelatinase. Cancer Res 53: 3154-3158

Katzmann DJ, Babst M, Emr SD (2001) Ubiquitin-dependent sorting into the multivesicular body pathway requires the function of a conserved endosomal protein sorting complex, ESCRT-I. Cell 106: $145-155$

Kawamoto T, Ohga N, Akiyama K, Hirata N, Kitahara S, Maishi N, Osawa T, Yamamoto K, Kondoh M, Shindoh M, Hida Y, Hida K (2012) Tumor-Derived Microvesicles Induce Proangiogenic Phenotype in Endothelial Cells via Endocytosis. Plos One 7

Keller S, Konig AK, Marme F, Runz S, Wolterink S, Koensgen D, Mustea A, Sehouli J, Altevogt P (2009) Systemic presence and tumor-growth promoting effect of ovarian carcinoma released exosomes. Cancer Lett 278: 73-81

Kessenbrock K, Plaks V, Werb Z (2010) Matrix metalloproteinases: regulators of the tumor microenvironment. Cell 141: 52-67

Klein CA, Seidl S, Petat-Dutter K, Offner S, Geigl JB, Schmidt-Kittler O, Wendler N, Passlick B, Huber RM, Schlimok G, Baeuerle PA, Riethmuller G (2002) Combined transcriptome and genome analysis of single micrometastatic cells. Nature biotechnology 20: 387-392

Koga K, Aoki M, Sameshima T, Hamasaki M, Egawa N, Seiki M, Toole BP, Suzumiya J, Nabeshima K (2011) Synthetic emmprin peptides inhibit tumor cell-fibroblast interaction-stimulated upregulation of MMP-2 and tumor cell invasion. Int J Oncol 39: 657-664

Koga K, Matsumoto K, Akiyoshi T, Kubo M, Yamanaka N, Tasaki A, Nakashima H, Nakamura M, Kuroki S, Tanaka M, Katano M (2005) Purification, characterization and biological significance of tumor-derived exosomes. Anticancer research 25: 3703-3707

Komekado H, Yamamoto H, Chiba T, Kikuchi A (2007) Glycosylation and palmitoylation of Wnt-3a are coupled to produce an active form of Wnt-3a. Genes to cells : devoted to molecular \& cellular mechanisms 12: 521-534

Krauel K, Hackbarth C, Furll B, Greinacher A (2012) Heparin-induced thrombocytopenia: in vitro studies on the interaction of dabigatran, rivaroxaban, and low-sulfated heparin, with platelet factor 4 and anti-PF4/heparin antibodies. Blood 119: 1248-1255

Kremenevskaja N, von Wasielewski R, Rao AS, Schofl C, Andersson T, Brabant G (2005) Wnt-5a has tumor suppressor activity in thyroid carcinoma. Oncogene 24: 2144-2154

Kufe DW (2009) Mucins in cancer: function, prognosis and therapy. Nature reviews Cancer 9: 874885

Kuhl M, Sheldahl LC, Malbon CC, Moon RT (2000) Ca(2+)/calmodulin-dependent protein kinase II is stimulated by Wnt and Frizzled homologs and promotes ventral cell fates in Xenopus. The Journal of biological chemistry 275: 12701-12711

Kumar S, Jiang MS, Adams JL, Lee JC (1999) Pyridinylimidazole compound SB 203580 inhibits the activity but not the activation of p38 mitogen-activated protein kinase. Biochemical and biophysical research communications 263: 825-831

Kurayoshi M, Yamamoto H, Izumi S, Kikuchi A (2007) Post-translational palmitoylation and glycosylation of Wnt-5a are necessary for its signalling. The Biochemical journal 402: 515523 
Laemmli UK (1970) Cleavage of structural proteins during the assembly of the head of bacteriophage T4. Nature 227: 680-685

Lechner D, Weltermann A (2008) Circulating tissue factor-exposing microparticles. Thrombosis research 122 Suppl 1: S47-54

Lee YT (1983) Breast carcinoma: pattern of metastasis at autopsy. Journal of surgical oncology 23: $175-180$

Lewis CE, Pollard JW (2006) Distinct role of macrophages in different tumor microenvironments. Cancer Res 66: 605-612

Li Y, Xu J, Chen L, Zhong WD, Zhang Z, Mi L, Zhang Y, Liao CG, Bian HJ, Jiang JL, Yang XM, Li XY, Fan CM, Zhu P, Fu L, Chen ZN (2009) HAb18G (CD147), a cancer-associated biomarker and its role in cancer detection. Histopathology 54: 677-687

Liang H, Chen Q, Coles AH, Anderson SJ, Pihan G, Bradley A, Gerstein R, Jurecic R, Jones SN (2003) Wnt5a inhibits B cell proliferation and functions as a tumor suppressor in hematopoietic tissue. Cancer cell 4: 349-360

Liao CG, Kong LM, Song F, Xing JL, Wang LX, Sun ZJ, Tang H, Yao H, Zhang Y, Wang L, Wang Y, Yang XM, Li Y, Chen ZN (2011) Characterization of Basigin Isoforms and the Inhibitory Function of Basigin-3 in Human Hepatocellular Carcinoma Proliferation and Invasion. Mol Cell Biol 31: 2591-2604

Liepins A (1983) Possible role of microtubules in tumor cell surface membrane shedding, permeability, and lympholysis. Cellular immunology 76: 120-128

Lim M, Martinez T, Jablons D, Cameron R, Guo HM, Toole B, Li JD, Basbaum C (1998) Tumorderived EMMPRIN (extracellular matrix metalloproteinase inducer) stimulates collagenase transcription through MAPK p38. Febs Lett 441: 88-92

Lin EY, Li JF, Gnatovskiy L, Deng Y, Zhu L, Grzesik DA, Qian H, Xue XN, Pollard JW (2006) Macrophages regulate the angiogenic switch in a mouse model of breast cancer. Cancer Res 66: $11238-11246$

Lin EY, Nguyen AV, Russell RG, Pollard JW (2001) Colony-stimulating factor 1 promotes progression of mammary tumors to malignancy. The Journal of experimental medicine 193: 727-740

Lingwood D, Simons K (2010) Lipid rafts as a membrane-organizing principle. Science 327: 46-50

Liu C, Yu S, Zinn K, Wang J, Zhang L, Jia Y, Kappes JC, Barnes S, Kimberly RP, Grizzle WE, Zhang HG (2006) Murine mammary carcinoma exosomes promote tumor growth by suppression of NK cell function. J Immunol 176: 1375-1385

Losche W, Scholz T, Temmler U, Oberle V, Claus RA (2004) Platelet-derived microvesicles transfer tissue factor to monocytes but not to neutrophils. Platelets 15: 109-115

Lowry OH, Rosebrough NJ, Farr AL, Randall RJ (1951) Protein measurement with the Folin phenol reagent. The Journal of biological chemistry 193: 265-275

Luckman SP, Coxon FP, Ebetino FH, Russell RG, Rogers MJ (1998a) Heterocycle-containing bisphosphonates cause apoptosis and inhibit bone resorption by preventing protein prenylation: evidence from structure-activity relationships in $\mathbf{J 7 7 4}$ macrophages. Journal of bone and mineral research : the official journal of the American Society for Bone and Mineral Research 13: 1668-1678

Luckman SP, Hughes DE, Coxon FP, Graham R, Russell G, Rogers MJ (1998b) Nitrogen-containing bisphosphonates inhibit the mevalonate pathway and prevent post-translational prenylation of GTP-binding proteins, including Ras. Journal of bone and mineral research : the official journal of the American Society for Bone and Mineral Research 13: 581-589

MacKenzie A, Wilson HL, Kiss-Toth E, Dower SK, North RA, Surprenant A (2001) Rapid secretion of interleukin-1beta by microvesicle shedding. Immunity 15: 825-835 
Maguire CA, Balaj L, Sivaraman S, Crommentuijn MH, Ericsson M, Mincheva-Nilsson L, Baranov V, Gianni D, Tannous BA, Sena-Esteves M, Breakefield XO, Skog J (2012) Microvesicleassociated AAV vector as a novel gene delivery system. Molecular therapy: the journal of the American Society of Gene Therapy 20: 960-971

Maley F, Trimble RB, Tarentino AL, Plummer TH, Jr. (1989) Characterization of glycoproteins and their associated oligosaccharides through the use of endoglycosidases. Analytical biochemistry 180: $195-204$

Malone KE, Daling JR, Thompson JD, O'Brien CA, Francisco LV, Ostrander EA (1998) BRCA1 mutations and breast cancer in the general population: analyses in women before age 35 years and in women before age 45 years with first-degree family history. JAMA : the journal of the American Medical Association 279: 922-929

Mannel DN, Orosz P, Hafner M, Falk W (1994) Mechanisms involved in metastasis enhanced by inflammatory mediators. Circulatory shock 44: 9-13

Mantovani A, Bottazzi B, Colotta F, Sozzani S, Ruco L (1992) The origin and function of tumorassociated macrophages. Immunology today 13: 265-270

Mantovani A, Sica A, Sozzani S, Allavena P, Vecchi A, Locati M (2004) The chemokine system in diverse forms of macrophage activation and polarization. Trends in immunology 25: 677-686

Mantovani A, Sozzani S, Locati M, Allavena P, Sica A (2002) Macrophage polarization: tumorassociated macrophages as a paradigm for polarized M2 mononuclear phagocytes. Trends in immunology 23: 549-555

Marshall OJ (2004) PerlPrimer: cross-platform, graphical primer design for standard, bisulphite and real-time PCR. Bioinformatics 20: 2471-2472

Martin SJ, Reutelingsperger CP, McGahon AJ, Rader JA, van Schie RC, LaFace DM, Green DR (1995) Early redistribution of plasma membrane phosphatidylserine is a general feature of apoptosis regardless of the initiating stimulus: inhibition by overexpression of $\mathrm{Bcl}-2$ and $\mathrm{Abl}$. The Journal of experimental medicine 182: 1545-1556

Matrisian LM, Bowden GT, Krieg P, Furstenberger G, Briand JP, Leroy P, Breathnach R (1986) The mRNA coding for the secreted protease transin is expressed more abundantly in malignant than in benign tumors. Proc Natl Acad Sci U S A 83: 9413-9417

Mause SF, Weber C (2010) Microparticles: protagonists of a novel communication network for intercellular information exchange. Circ Res 107: 1047-1057

McDonald SL, Silver A (2009) The opposing roles of Wnt-5a in cancer. British journal of cancer 101: 209-214

McKay MJ, Langlands AO (1992) Prognostic factors in breast cancer. The New England journal of medicine 327: 1317; author reply 1318

McNiven MA, Cao H, Pitts KR, Yoon Y (2000) The dynamin family of mechanoenzymes: pinching in new places. Trends in biochemical sciences 25: 115-120

Menck K, Behme D, Reiling N, Binder C, Pukrop T, Klemm F (2014a) Isolation of human monocytes by double gradient centrifugation and their differentiation to macrophages in Teflon-coated cell culture bags. Journal of visualized experiments

Menck K, Klemm F, Gross JC, Pukrop T, Wenzel D, Binder C (2013) Induction and transport of Wnt 5a during macrophage-induced malignant invasion is mediated by two types of extracellular vesicles. Oncotarget 4: 2057-2066

Menck K, Scharf C, Bleckmann A, Dyck L, Wenzel D, Siam L, Pukrop T, Binder C, Klemm F (2014b) Tumor-derived microvesicles mediate human breast cancer invasion through differentially glycosylated EMMPRIN.

Mikels AJ, Nusse R (2006) Purified Wnt5a protein activates or inhibits beta-catenin-TCF signaling depending on receptor context. PLoS biology 4: e115 
Mittelbrunn M, Gutierrez-Vazquez C, Villarroya-Beltri C, Gonzalez S, Sanchez-Cabo F, Gonzalez MA, Bernad A, Sanchez-Madrid F (2011) Unidirectional transfer of microRNA-loaded exosomes from T cells to antigen-presenting cells. Nature communications 2: 282

Miyoshi H, Umeshita K, Sakon M, Imajoh-Ohmi S, Fujitani K, Gotoh M, Oiki E, Kambayashi J, Monden M (1996) Calpain activation in plasma membrane bleb formation during tert-butyl hydroperoxide-induced rat hepatocyte injury. Gastroenterology 110: 1897-1904

Montecalvo A, Larregina AT, Shufesky WJ, Stolz DB, Sullivan ML, Karlsson JM, Baty CJ, Gibson GA, Erdos G, Wang Z, Milosevic J, Tkacheva OA, Divito SJ, Jordan R, Lyons-Weiler J, Watkins SC, Morelli AE (2012) Mechanism of transfer of functional microRNAs between mouse dendritic cells via exosomes. Blood 119: 756-766

Morel O, Toti F, Hugel B, Bakouboula B, Camoin-Jau L, Dignat-George F, Freyssinet JM (2006) Procoagulant microparticles: disrupting the vascular homeostasis equation? Arteriosclerosis, thrombosis, and vascular biology 26: 2594-2604

Morrell NT, Leucht P, Zhao L, Kim JB, ten Berge D, Ponnusamy K, Carre AL, Dudek H, Zachlederova M, McElhaney M, Brunton S, Gunzner J, Callow M, Polakis P, Costa M, Zhang XM, Helms JA, Nusse R (2008) Liposomal packaging generates Wnt protein with in vivo biological activity. Plos One 3: e2930

Moskovich O, Fishelson Z (2007) Live cell imaging of outward and inward vesiculation induced by the complement c5b-9 complex. The Journal of biological chemistry 282: 29977-29986

Mosmann T (1983) Rapid colorimetric assay for cellular growth and survival: application to proliferation and cytotoxicity assays. J Immunol Methods 65: 55-63

Mosser DM (2003) The many faces of macrophage activation. J Leukoc Biol 73: 209-212

Muralidharan-Chari V, Clancy J, Plou C, Romao M, Chavrier P, Raposo G, D'Souza-Schorey C (2009) ARF6-Regulated Shedding of Tumor Cell-Derived Plasma Membrane Microvesicles. Curr Biol 19: 1875-1885

Muralidharan-Chari V, Clancy JW, Sedgwick A, D'Souza-Schorey C (2010) Microvesicles: mediators of extracellular communication during cancer progression. J Cell Sci 123: 1603-1611

Nabeshima K, Iwasaki H, Koga K, Hojo H, Suzumiya J, Kikuchi M (2006) Emmprin (basigin/CD147): matrix metalloproteinase modulator and multifunctional cell recognition molecule that plays a critical role in cancer progression. Pathology international 56: 359-367

Nabeshima K, Suzumiya J, Nagano M, Ohshima K, Toole BP, Tamura K, Iwasaki H, Kikuchi M (2004) Emmprin, a cell surface inducer of matrix metalloproteinases (MMPs), is expressed in T-cell lymphomas. The Journal of pathology 202: 341-351

Nitzki F, Zibat A, Konig S, Wijgerde M, Rosenberger A, Brembeck FH, Carstens PO, Frommhold A, Uhmann A, Klingler S, Reifenberger J, Pukrop T, Aberger F, Schulz-Schaeffer W, Hahn H (2010) Tumor stroma-derived Wnt5a induces differentiation of basal cell carcinoma of Ptchmutant mice via CaMKII. Cancer Res 70: 2739-2748

Nusse R, Varmus HE (1982) Many tumors induced by the mouse mammary tumor virus contain a provirus integrated in the same region of the host genome. Cell 31: 99-109

Obregon C, Rothen-Rutishauser B, Gitahi SK, Gehr P, Nicod LP (2006) Exovesicles from human activated dendritic cells fuse with resting dendritic cells, allowing them to present alloantigens. Am J Pathol 169: 2127-2136

Olson DJ, Papkoff J (1994) Regulated expression of Wnt family members during proliferation of C57mg mammary cells. Cell growth \& differentiation : the molecular biology journal of the American Association for Cancer Research 5: 197-206

Ostrowski M, Carmo NB, Krumeich S, Fanget I, Raposo G, Savina A, Moita CF, Schauer K, Hume AN, Freitas RP, Goud B, Benaroch P, Hacohen N, Fukuda M, Desnos C, Seabra MC, Darchen 
F, Amigorena S, Moita LF, Thery C (2010) Rab27a and Rab27b control different steps of the exosome secretion pathway. Nat Cell Biol 12: 19-30; sup pp 11-13

Paget S (1889) The distribution of secondary growths in cancer of the breast. Lancet 1: 571-573

Pan BT, Johnstone RM (1983) Fate of the transferrin receptor during maturation of sheep reticulocytes in vitro: selective externalization of the receptor. Cell 33: 967-978

Park S, Ahn HK, Park LC, Hwang DW, Ji JH, Maeng CH, Cho SH, Lee JY, Park KT, Ahn JS, Park YH, Im YH (2012) Implications of different CA 15-3 levels according to breast cancer subtype at initial diagnosis of recurrent or metastatic breast cancer. Oncology 82: 180-187

Parolini I, Federici C, Raggi C, Lugini L, Palleschi S, De Milito A, Coscia C, Iessi E, Logozzi M, Molinari A, Colone M, Tatti M, Sargiacomo M, Fais S (2009) Microenvironmental pH is a key factor for exosome traffic in tumor cells. The Journal of biological chemistry 284: 3421134222

Peinado H, Aleckovic M, Lavotshkin S, Matei I, Costa-Silva B, Moreno-Bueno G, Hergueta-Redondo M, Williams C, Garcia-Santos G, Ghajar C, Nitadori-Hoshino A, Hoffman C, Badal K, Garcia BA, Callahan MK, Yuan J, Martins VR, Skog J, Kaplan RN, Brady MS, Wolchok JD, Chapman PB, Kang Y, Bromberg J, Lyden D (2012) Melanoma exosomes educate bone marrow progenitor cells toward a pro-metastatic phenotype through MET. Nature medicine 18: $883-891$

Pereira C, Schaer DJ, Bachli EB, Kurrer MO, Schoedon G (2008) Wnt5A/CaMKII signaling contributes to the inflammatory response of macrophages and is a target for the antiinflammatory action of activated protein $\mathrm{C}$ and interleukin-10. Arterioscl Throm Vas 28: 504-510

Perou CM, Sorlie T, Eisen MB, van de Rijn M, Jeffrey SS, Rees CA, Pollack JR, Ross DT, Johnsen H, Akslen LA, Fluge O, Pergamenschikov A, Williams C, Zhu SX, Lonning PE, Borresen-Dale AL, Brown PO, Botstein D (2000) Molecular portraits of human breast tumours. Nature 406: 747-752

Peterson GL (1979) Review of the Folin phenol protein quantitation method of Lowry, Rosebrough, Farr and Randall. Analytical biochemistry 100: 201-220

Pilzer D, Gasser O, Moskovich O, Schifferli JA, Fishelson Z (2005) Emission of membrane vesicles: roles in complement resistance, immunity and cancer. Springer seminars in immunopathology 27: 375-387

Pizzirani C, Ferrari D, Chiozzi P, Adinolfi E, Sandona D, Savaglio E, Di Virgilio F (2007) Stimulation of $\mathrm{P} 2$ receptors causes release of IL-1beta-loaded microvesicles from human dendritic cells. Blood 109: 3856-3864

Pluskota E, Woody NM, Szpak D, Ballantyne CM, Soloviev DA, Simon DI, Plow EF (2008) Expression, activation, and function of integrin alphaMbeta2 (Mac-1) on neutrophil-derived microparticles. Blood 112: 2327-2335

Pollard JW (2004) Tumour-educated macrophages promote tumour progression and metastasis. Nature reviews Cancer 4: 71-78

Port F, Hausmann G, Basler K (2011) A genome-wide RNA interference screen uncovers two p24 proteins as regulators of Wingless secretion. EMBO reports 12: 1144-1152

Port F, Kuster M, Herr P, Furger E, Banziger C, Hausmann G, Basler K (2008) Wingless secretion promotes and requires retromer-dependent cycling of Wntless. Nat Cell Biol 10: 178-185

Poste G, Fidler IJ (1980) The pathogenesis of cancer metastasis. Nature 283: 139-146

Poste G, Nicolson GL (1980) Arrest and metastasis of blood-borne tumor cells are modified by fusion of plasma membrane vesicles from highly metastatic cells. Proc Natl Acad Sci U S A 77: 399403 
Pourreyron C, Reilly L, Proby C, Panteleyev A, Fleming C, McLean K, South AP, Foerster J (2012) Wnt5a is strongly expressed at the leading edge in non-melanoma skin cancer, forming active gradients, while canonical Wnt signalling is repressed. Plos One 7: e31827

Prieve MG, Moon RT (2003) Stromelysin-1 and mesothelin are differentially regulated by Wnt-5a and Wnt-1 in C57mg mouse mammary epithelial cells. BMC developmental biology 3: 2

Pukrop T, Binder C (2008) The complex pathways of Wnt 5a in cancer progression. J Mol Med-Jmm 86: $259-266$

Pukrop T, Klemm F, Hagemann T, Gradl D, Schulz M, Siemes S, Trumper L, Binder C (2006) Wnt 5a signaling is critical for macrophage-induced invasion of breast cancer cell lines. P Natl Acad Sci USA 103: 5454-5459

Qian BZ, Pollard JW (2010) Macrophage diversity enhances tumor progression and metastasis. Cell 141: $39-51$

Rana S, Malinowska K, Zoller M (2013) Exosomal tumor microRNA modulates premetastatic organ cells. Neoplasia 15: 281-295

Rao TP, Kuhl M (2010) An updated overview on Wnt signaling pathways: a prelude for more. Circ Res 106: 1798-1806

Raposo G, Stoorvogel W (2013) Extracellular vesicles: exosomes, microvesicles, and friends. J Cell Biol 200: 373-383

Raposo G, Tenza D, Mecheri S, Peronet R, Bonnerot C, Desaymard C (1997) Accumulation of major histocompatibility complex class II molecules in mast cell secretory granules and their release upon degranulation. Mol Biol Cell 8: 2631-2645

Ratajczak J, Miekus K, Kucia M, Zhang J, Reca R, Dvorak P, Ratajczak MZ (2006) Embryonic stem cell-derived microvesicles reprogram hematopoietic progenitors: evidence for horizontal transfer of mRNA and protein delivery. Leukemia 20: 847-856

Redzic JS, Kendrick AA, Bahmed K, Dahl KD, Pearson CG, Robinson WA, Robinson SE, Graner MW, Eisenmesser EZ (2013) Extracellular vesicles secreted from cancer cell lines stimulate secretion of MMP-9, IL-6, TGF-beta1 and EMMPRIN. Plos One 8: e71225

Reed JC (2000) Mechanisms of apoptosis. Am J Pathol 157: 1415-1430

Riethdorf S, Fritsche H, Muller V, Rau T, Schindlbeck C, Rack B, Janni W, Coith C, Beck K, Janicke F, Jackson S, Gornet T, Cristofanilli M, Pantel K (2007) Detection of circulating tumor cells in peripheral blood of patients with metastatic breast cancer: a validation study of the CellSearch system. Clinical cancer research : an official journal of the American Association for Cancer Research 13: 920-928

Riethdorf S, Reimers N, Assmann V, Kornfeld JW, Terracciano L, Sauter G, Pantel K (2006) High incidence of EMMPRIN expression in human tumors. Int J Cancer 119: 1800-1810

Rietkotter E, Menck K, Bleckmann A, Farhat K, Schaffrinski M, Schulz M, Hanisch UK, Binder C, Pukrop T (2013) Zoledronic acid inhibits macrophage/microglia-assisted breast cancer cell invasion. Oncotarget 4: 1449-1460

Ripka S, Konig A, Buchholz M, Wagner M, Sipos B, Kloppel G, Downward J, Gress T, Michl P (2007) WNT5A--target of CUTL1 and potent modulator of tumor cell migration and invasion in pancreatic cancer. Carcinogenesis 28: 1178-1187

Saini KS, Azim HA, Jr., Metzger-Filho O, Loi S, Sotiriou C, de Azambuja E, Piccart M (2011) Beyond trastuzumab: new treatment options for HER2-positive breast cancer. Breast 20 Suppl 3: $\mathrm{S} 20-27$

Sato T, Ota T, Watanabe M, Imada K, Nomizu M, Ito A (2009) Identification of an active site of EMMPRIN for the augmentation of matrix metalloproteinase-1 and -3 expression in a coculture of human uterine cervical carcinoma cells and fibroblasts. Gynecol Oncol 114: 337342 
Savina A, Fader CM, Damiani MT, Colombo MI (2005) Rab11 promotes docking and fusion of multivesicular bodies in a calcium-dependent manner. Traffic 6: 131-143

Schmidt M, Hasenclever D, Schaeffer M, Boehm D, Cotarelo C, Steiner E, Lebrecht A, Siggelkow W, Weikel W, Schiffer-Petry I, Gebhard S, Pilch H, Gehrmann M, Lehr HA, Koelbl H, Hengstler JG, Schuler M (2008a) Prognostic effect of epithelial cell adhesion molecule overexpression in untreated node-negative breast cancer. Clinical cancer research : an official journal of the American Association for Cancer Research 14: 5849-5855

Schmidt R, Bueltmann A, Fischel S, Gillitzer A, Cullen P, Walch A, Jost P, Ungerer M, Tolley ND, Lindemann S, Gawaz M, Schoemig A, May AE (2008b) Extracellular matrix metalloproteinase inducer (CD147) is a novel receptor on platelets, activates platelets, and augments nuclear factor kappa B-dependent inflammation in monocytes. Circ Res 102: 302309

Schwab W, Harada H, Goetz W, Nowicki M, Witt M, Kasper M, Barth K (2007) Immunocytochemical and biochemical detection of EMMPRIN in the rat tooth germ: differentiation-dependent co-expression with MMPs and co-localization with caveolin-1 in membrane rafts of dental epithelial cells. Histochemistry and cell biology 128: 195-203

Seizer P, Schonberger T, Schott M, Lang MR, Langer HF, Bigalke B, Kramer BF, Borst O, Daub K, Heidenreich O, Schmidt R, Lindemann S, Herouy Y, Gawaz M, May AE (2010) EMMPRIN and its ligand cyclophilin A regulate MT1-MMP, MMP-9 and M-CSF during foam cell formation. Atherosclerosis 209: 51-57

Semenov MV, Zhang X, He X (2008) DKK1 antagonizes Wnt signaling without promotion of LRP6 internalization and degradation. The Journal of biological chemistry 283: 21427-21432

Senkus E, Kyriakides S, Penault-Llorca F, Poortmans P, Thompson A, Zackrisson S, Cardoso F, Group EGW (2013) Primary breast cancer: ESMO Clinical Practice Guidelines for diagnosis, treatment and follow-up. Annals of oncology : official journal of the European Society for Medical Oncology / ESMO 24 Suppl 6: vi7-23

Setty BN, Kulkarni S, Rao AK, Stuart MJ (2000) Fetal hemoglobin in sickle cell disease: relationship to erythrocyte phosphatidylserine exposure and coagulation activation. Blood 96: 1119-1124

Shedden K, Xie XT, Chandaroy P, Chang YT, Rosania GR (2003) Expulsion of small molecules in vesicles shed by cancer cells: association with gene expression and chemosensitivity profiles. Cancer Res 63: 4331-4337

Shigemura N, Kiyoshima T, Sakai T, Matsuo K, Momoi T, Yamaza H, Kobayashi I, Wada H, Akamine A, Sakai H (2001) Localization of activated caspase-3-positive and apoptotic cells in the developing tooth germ of the mouse lower first molar. The Histochemical journal 33: 253258

Shih J-Y, Yuan A, Chen JJ-W, Yang P-C (2006) Tumor-Associated Macrophage: Its Role in Cancer Invasion and Metastasis. JOCM 2: 101-106

Sidhu SS, Mengistab AT, Tauscher AN, LaVail J, Basbaum C (2004) The microvesicle as a vehicle for EMMPRIN in tumor-stromal interactions. Oncogene 23: 956-963

Sims PJ, Wiedmer T, Esmon CT, Weiss HJ, Shattil SJ (1989) Assembly of the platelet prothrombinase complex is linked to vesiculation of the platelet plasma membrane. Studies in Scott syndrome: an isolated defect in platelet procoagulant activity. The Journal of biological chemistry 264: 17049-17057

Skog J, Wurdinger T, van Rijn S, Meijer DH, Gainche L, Sena-Esteves M, Curry WT, Jr., Carter BS, Krichevsky AM, Breakefield XO (2008) Glioblastoma microvesicles transport RNA and proteins that promote tumour growth and provide diagnostic biomarkers. Nat Cell Biol 10: $1470-1476$ 
Slamon DJ, Clark GM, Wong SG, Levin WJ, Ullrich A, McGuire WL (1987) Human breast cancer: correlation of relapse and survival with amplification of the HER-2/neu oncogene. Science 235: $177-182$

Sluijter JPG, Pulskens WPC, Schoneveld AH, Velema E, Strijder CF, Moll F, de Vries JP, Verheijen J, Hanemaaijer R, de Kleijn DPV, Pasterkamp G (2006) Matrix metalloproteinase 2 is associated with stable and matrix metalloproteinases 8 and 9 with vulnerable carotid atherosclerotic lesions - A study in human endarterectomy specimen pointing to a role for different extracellular matrix metalloproteinase inducer glycosylation forms. Stroke 37: 235239

Smalley DM, Sheman NE, Nelson K, Theodorescu D (2008) Isolation and identification of potential urinary microparticle biomarkers of bladder cancer. J Proteome Res 7: 2088-2096

Smith K, Bui TD, Poulsom R, Kaklamanis L, Williams G, Harris AL (1999) Up-regulation of macrophage wnt gene expression in adenoma-carcinoma progression of human colorectal cancer. British journal of cancer 81: 496-502

Solinas G, Germano G, Mantovani A, Allavena P (2009) Tumor-associated macrophages (TAM) as major players of the cancer-related inflammation. J Leukoc Biol 86: 1065-1073

Solis GP, Luchtenborg AM, Katanaev VL (2013) Wnt secretion and gradient formation. International journal of molecular sciences 14: 5130-5145

Sorlie T, Perou CM, Tibshirani R, Aas T, Geisler S, Johnsen H, Hastie T, Eisen MB, van de Rijn M, Jeffrey SS, Thorsen T, Quist H, Matese JC, Brown PO, Botstein D, Lonning PE, BorresenDale AL (2001) Gene expression patterns of breast carcinomas distinguish tumor subclasses with clinical implications. Proc Natl Acad Sci U S A 98: 10869-10874

Soule HD, Vazquez J, Long A, Albert S, Brennan M (1973) Human Cell Line from a Pleural Effusion Derived from a Breast Carcinoma. J Natl Cancer I 51: 1409-1416

Spano JP, Fagard R, Soria JC, Rixe O, Khayat D, Milano G (2005) Epidermal growth factor receptor signaling in colorectal cancer: preclinical data and therapeutic perspectives. Annals of oncology : official journal of the European Society for Medical Oncology / ESMO 16: 189194

Stacker SA, Baldwin ME, Achen MG (2002) The role of tumor lymphangiogenesis in metastatic spread. Faseb J 16: 922-934

Sternlicht MD, Werb Z (2001) How matrix metalloproteinases regulate cell behavior. Annual review of cell and developmental biology 17: 463-516

Stoelcker B, Hafner M, Orosz P, Nieswandt B, Mannel DN (1995) Role of adhesion molecules and platelets in TNF-induced adhesion of tumor cells to endothelial cells: implications for experimental metastasis. Journal of inflammation 46: 155-167

Stoorvogel W, Kleijmeer MJ, Geuze HJ, Raposo G (2002) The biogenesis and functions of exosomes. Traffic 3: 321-330

Strauss K, Goebel C, Runz H, Mobius W, Weiss S, Feussner I, Simons M, Schneider A (2010) Exosome secretion ameliorates lysosomal storage of cholesterol in Niemann-Pick type C disease. The Journal of biological chemistry 285: 26279-26288

Sun J, Hemler ME (2001) Regulation of MMP-1 and MMP-2 production through CD147/extracellular matrix metalloproteinase inducer interactions. Cancer Res 61: 2276-2281

Svensson KJ, Christianson HC, Wittrup A, Bourseau-Guilmain E, Lindqvist E, Svensson LM, Morgelin M, Belting M (2013) Exosome uptake depends on ERK1/2-heat shock protein 27 signaling and lipid Raft-mediated endocytosis negatively regulated by caveolin-1. The Journal of biological chemistry 288: 17713-17724

Szabo E (2003) MUC1 expression in lung cancer. Methods in molecular medicine 74: 251-258 
Tamai K, Semenov M, Kato Y, Spokony R, Liu C, Katsuyama Y, Hess F, Saint-Jeannet JP, He X (2000) LDL-receptor-related proteins in Wnt signal transduction. Nature 407: 530-535

Tanaka K, Okabayashi K, Asashima M, Perrimon N, Kadowaki T (2000) The evolutionarily conserved porcupine gene family is involved in the processing of the Wnt family. European journal of biochemistry / FEBS 267: 4300-4311

Tang W, Chang SB, Hemler ME (2004a) Links between CD147 function, glycosylation, and caveolin1. Mol Biol Cell 15: 4043-4050

Tang Y, Kesavan P, Nakada MT, Yan L (2004b) Tumor-stroma interaction: positive feedback regulation of extracellular matrix metalloproteinase inducer (EMMPRIN) expression and matrix metalloproteinase-dependent generation of soluble EMMPRIN. Molecular cancer research: MCR 2: 73-80

Tang Y, Nakada MT, Kesavan P, McCabe F, Millar H, Rafferty P, Bugelski P, Yan L (2005) Extracellular matrix metalloproteinase inducer stimulates tumor angiogenesis by elevating vascular endothelial cell growth factor and matrix metalloproteinases. Cancer Res 65: 31933199

Taraboletti G, D'Ascenzo S, Giusti I, Marchetti D, Borsotti P, Millimaggi D, Giavazzi R, Pavan A, Dolo V (2006) Bioavailability of VEGF in tumor-shed vesicles depends on vesicle burst induced by acidic pH. Neoplasia 8: 96-103

Taylor DD, Gercel-Taylor C (2005) Tumour-derived exosomes and their role in cancer-associated Tcell signalling defects. British journal of cancer 92: 305-311

Taylor DD, Gercel-Taylor C (2008) MicroRNA signatures of tumor-derived exosomes as diagnostic biomarkers of ovarian cancer. Gynecol Oncol 110: 13-21

Tesselaar ME, Romijn FP, Van Der Linden IK, Prins FA, Bertina RM, Osanto S (2007) Microparticleassociated tissue factor activity: a link between cancer and thrombosis? Journal of thrombosis and haemostasis : JTH 5: 520-527

Thery C, Amigorena S, Raposo G, Clayton A (2006) Isolation and characterization of exosomes from cell culture supernatants and biological fluids. Current protocols in cell biology / editorial board, Juan S Bonifacino [et al] Chapter 3: Unit 322

Thery C, Boussac M, Veron P, Ricciardi-Castagnoli P, Raposo G, Garin J, Amigorena S (2001) Proteomic analysis of dendritic cell-derived exosomes: a secreted subcellular compartment distinct from apoptotic vesicles. J Immunol 166: 7309-7318

Thery C, Zitvogel L, Amigorena S (2002) Exosomes: composition, biogenesis and function. Nat Rev Immunol 2: 569-579

Tomaskovic-Crook E, Thompson EW, Thiery JP (2009) Epithelial to mesenchymal transition and breast cancer. Breast cancer research : BCR 11: 213

Toth B, Liebhardt S, Steinig K, Ditsch N, Rank A, Bauerfeind I, Spannagl M, Friese K, Reininger AJ (2008a) Platelet-derived microparticles and coagulation activation in breast cancer patients. Thrombosis and haemostasis 100: 663-669

Toth B, Nieuwland R, Liebhardt S, Ditsch N, Steinig K, Stieber P, Rank A, Gohring P, Thaler CJ, Friese K, Bauerfeind I (2008b) Circulating microparticles in breast cancer patients: a comparative analysis with established biomarkers. Anticancer research 28: 1107-1112

Trempe GL (1976) Human breast cancer in culture. Recent results in cancer research Fortschritte der Krebsforschung Progres dans les recherches sur le cancer: 33-41

Turiak L, Misjak P, Szabo TG, Aradi B, Paloczi K, Ozohanics O, Drahos L, Kittel A, Falus A, Buzas EI, Vekey K (2011) Proteomic characterization of thymocyte-derived microvesicles and apoptotic bodies in BALB/c mice. Journal of proteomics 74: 2025-2033 
Turpeenniemi-Hujanen T, Thorgeirsson UP, Hart IR, Grant SS, Liotta LA (1985) Expression of collagenase IV (basement membrane collagenase) activity in murine tumor cell hybrids that differ in metastatic potential. J Natl Cancer Inst 75: 99-103

Unlu M, Morgan ME, Minden JS (1997) Difference gel electrophoresis: a single gel method for detecting changes in protein extracts. Electrophoresis 18: 2071-2077

Valadi H, Ekstrom K, Bossios A, Sjostrand M, Lee JJ, Lotvall JO (2007) Exosome-mediated transfer of mRNAs and microRNAs is a novel mechanism of genetic exchange between cells. Nat Cell Biol 9: 654-659

Valencia J, Hernandez-Lopez C, Martinez VG, Hidalgo L, Zapata AG, Vicente A, Varas A, Sacedon $\mathrm{R}$ (2011) Wnt5a skews dendritic cell differentiation to an unconventional phenotype with tolerogenic features. J Immunol 187: 4129-4139

Valenti R, Huber V, Filipazzi P, Pilla L, Sovena G, Villa A, Corbelli A, Fais S, Parmiani G, Rivoltini L (2006) Human tumor-released microvesicles promote the differentiation of myeloid cells with transforming growth factor-beta-mediated suppressive activity on T lymphocytes. Cancer Res 66: 9290-9298

van de Wetering M, Cavallo R, Dooijes D, van Beest M, van Es J, Loureiro J, Ypma A, Hursh D, Jones T, Bejsovec A, Peifer M, Mortin M, Clevers H (1997) Armadillo coactivates transcription driven by the product of the Drosophila segment polarity gene dTCF. Cell 88: 789-799

Van der Haegen BA, Shay JW (1993) Immortalization of human mammary epithelial cells by SV40 large T-antigen involves a two step mechanism. In vitro cellular \& developmental biology : journal of the Tissue Culture Association 29A: 180-182

van der Pol E, Boing AN, Harrison P, Sturk A, Nieuwland R (2012) Classification, functions, and clinical relevance of extracellular vesicles. Pharmacological reviews 64: 676-705

van Niel G, Porto-Carreiro I, Simoes S, Raposo G (2006) Exosomes: a common pathway for a specialized function. J Biochem 140: 13-21

VanWijk MJ, VanBavel E, Sturk A, Nieuwland R (2003) Microparticles in cardiovascular diseases. Cardiovasc Res 59: 277-287

von Schwedler UK, Stuchell M, Muller B, Ward DM, Chung HY, Morita E, Wang HE, Davis T, He GP, Cimbora DM, Scott A, Krausslich HG, Kaplan J, Morham SG, Sundquist WI (2003) The protein network of HIV budding. Cell 114: 701-713

Wallingford JB, Habas R (2005) The developmental biology of Dishevelled: an enigmatic protein governing cell fate and cell polarity. Development 132: 4421-4436

Wang J, Yang D, Li C, Shang S, Xiang J (2013) Expression of extracellular matrix metalloproteinase inducer glycosylation and caveolin-1 in healthy and inflamed human gingiva. Journal of periodontal research

Weber GF (2008) Molecular mechanisms of metastasis. Cancer Lett 270: 181-190

Weeraratna AT, Jiang Y, Hostetter G, Rosenblatt K, Duray P, Bittner M, Trent JM (2002) Wnt5a signaling directly affects cell motility and invasion of metastatic melanoma. Cancer cell 1: 279-288

Wehrli M, Dougan ST, Caldwell K, O'Keefe L, Schwartz S, Vaizel-Ohayon D, Schejter E, Tomlinson A, DiNardo S (2000) arrow encodes an LDL-receptor-related protein essential for Wingless signalling. Nature 407: 527-530

Weidle UH, Scheuer W, Eggle D, Klostermann S, Stockinger H (2010) Cancer-related issues of CD147. Cancer genomics \& proteomics 7: 157-169

Weigelt B, Peterse JL, van 't Veer LJ (2005) Breast cancer metastasis: markers and models. Nature reviews Cancer 5: 591-602 
Willert K, Brown JD, Danenberg E, Duncan AW, Weissman IL, Reya T, Yates JR, 3rd, Nusse R (2003) Wnt proteins are lipid-modified and can act as stem cell growth factors. Nature 423: $448-452$

Wirtz D, Konstantopoulos K, Searson PC (2011) The physics of cancer: the role of physical interactions and mechanical forces in metastasis. Nature reviews Cancer 11: 512-522

Witwer KW, Buzas EI, Bemis LT, Bora A, Lasser C, Lotvall J, Nolte-'t Hoen EN, Piper MG, Sivaraman S, Skog J, Thery C, Wauben MH, Hochberg F (2013) Standardization of sample collection, isolation and analysis methods in extracellular vesicle research. Journal of extracellular vesicles $\mathbf{2}$

Wolfers J, Lozier A, Raposo G, Regnault A, Thery C, Masurier C, Flament C, Pouzieux S, Faure F, Tursz T, Angevin E, Amigorena S, Zitvogel L (2001) Tumor-derived exosomes are a source of shared tumor rejection antigens for CTL cross-priming. Nature medicine 7: 297-303

Wong GT, Gavin BJ, McMahon AP (1994) Differential transformation of mammary epithelial cells by Wnt genes. Mol Cell Biol 14: 6278-6286

Wu C, Orozco C, Boyer J, Leglise M, Goodale J, Batalov S, Hodge CL, Haase J, Janes J, Huss JW, 3rd, Su AI (2009) BioGPS: an extensible and customizable portal for querying and organizing gene annotation resources. Genome biology 10: R130

Wyckoff JB, Wang Y, Lin EY, Li JF, Goswami S, Stanley ER, Segall JE, Pollard JW, Condeelis J (2007) Direct visualization of macrophage-assisted tumor cell intravasation in mammary tumors. Cancer Res 67: 2649-2656

Xie Y, Bai O, Yuan J, Chibbar R, Slattery K, Wei Y, Deng Y, Xiang J (2009) Tumor apoptotic bodies inhibit CTL responses and antitumor immunity via membrane-bound transforming growth factor-beta1 inducing CD8+ T-cell anergy and CD4+ Tr1 cell responses. Cancer Res 69: 7756-7766

Yamaguchi H, Takeo Y, Yoshida S, Kouchi Z, Nakamura Y, Fukami K (2009) Lipid rafts and caveolin-1 are required for invadopodia formation and extracellular matrix degradation by human breast cancer cells. Cancer Res 69: 8594-8602

Yamanaka H, Moriguchi T, Masuyama N, Kusakabe M, Hanafusa H, Takada R, Takada S, Nishida E (2002) JNK functions in the non-canonical Wnt pathway to regulate convergent extension movements in vertebrates. EMBO reports 3: 69-75

Yoshida S, Shibata M, Yamamoto S, Hagihara M, Asai N, Takahashi M, Mizutani S, Muramatsu T, Kadomatsu K (2000) Homo-oligomer formation by basigin, an immunoglobulin superfamily member, via its $\mathrm{N}$-terminal immunoglobulin domain. European journal of biochemistry / FEBS 267: 4372-4380

Yoshioka Y, Konishi Y, Kosaka N, Katsuda T, Kato T, Ochiya T (2013) Comparative marker analysis of extracellular vesicles in different human cancer types. Journal of extracellular vesicles 2

Zhong WD, Han ZD, He HC, Bi XC, Dai QS, Zhu G, Ye YK, Liang YX, Qin WJ, Zhang Z, Zeng GH, Chen ZN (2008) CD147, MMP-1, MMP-2 and MMP-9 protein expression as significant prognostic factors in human prostate cancer. Oncology 75: 230-236

Zitvogel L, Regnault A, Lozier A, Wolfers J, Flament C, Tenza D, Ricciardi-Castagnoli P, Raposo G, Amigorena $S$ (1998) Eradication of established murine tumors using a novel cell-free vaccine: dendritic cell-derived exosomes. Nature medicine 4: 594-600

Zucker S, Hymowitz M, Rollo EE, Mann R, Conner CE, Cao J, Foda HD, Tompkins DC, Toole BP (2001) Tumorigenic potential of extracellular matrix metalloproteinase inducer. Am J Pathol 158: $1921-1928$

Zwicker JI, Liebman HA, Neuberg D, Lacroix R, Bauer KA, Furie BC, Furie B (2009) Tumor-derived tissue factor-bearing microparticles are associated with venous thromboembolic events in malignancy. Clinical cancer research : an official journal of the American Association for Cancer Research 15: 6830-6840 


\section{Appendix}

\subsection{List of identified N-linked glycoproteins in 2D-DIGE gels}

Tab. 10: Proteins exclusively present on untreated $T-M V_{M}$

\begin{tabular}{|c|c|c|}
\hline Spot Nr. & Protein & Protein full name \\
\hline 70 & Anxa5 & Annexin A5 \\
\hline 86 & Actb & ß-actin \\
\hline 130 & Arpc2 & Actin-related protein 2/3 complex subunit 2 \\
\hline 135 & Csk21 & Casein kinase II subunit alpha \\
\hline 143 & K1c9 & Keratin, type I cytoskeletal 9 \\
\hline 143 & K2c1 & Keratin, type II cytoskeletal 1 \\
\hline 149 & Acts & $\alpha$-Actin, skeletal muscle \\
\hline 162 & Mfgm & milk fat globule-EGF factor 8 protein \\
\hline 164 & Serph & Serpin H1 \\
\hline 169 & Fa5 & Coagulation factor V \\
\hline 210 & Trap1 & Heat shock protein 75 kDa, mitochondrial \\
\hline 262 & Djb11 & DnaJ homolog subfamily B member 11 \\
\hline 263 & Arp2 & Actin-related protein 2 \\
\hline 306 & Atpb & ATP synthase subunit beta, mitochondrial \\
\hline 309 & Pdia1 & Protein disulfide-isomerase \\
\hline 370 & Hyou1 & hypoxia up-regulated 1 \\
\hline
\end{tabular}

Tab. 11: Proteins exclusively present on deglycosylated T-MV ${ }_{M}$

\begin{tabular}{|c|c|c|}
\hline Spot Nr. & Protein & Protein full name \\
\hline 26 & Hba & Hemoglobin subunit alpha \\
\hline 66 & Actb & Beta-actin \\
\hline 268 & Scmc1 & Calcium-binding mitochondrial carrier protein SCaMC-1 \\
\hline 342 & Mvp & Major vault protein \\
\hline 373 & Itav & Integrin alpha-V \\
\hline 381 & Basp1 & Brain acid soluble protein 1 \\
\hline 382 & Rcn2 & Reticulocalbin-2 \\
\hline
\end{tabular}




\subsection{LC-MS/MS analysis}
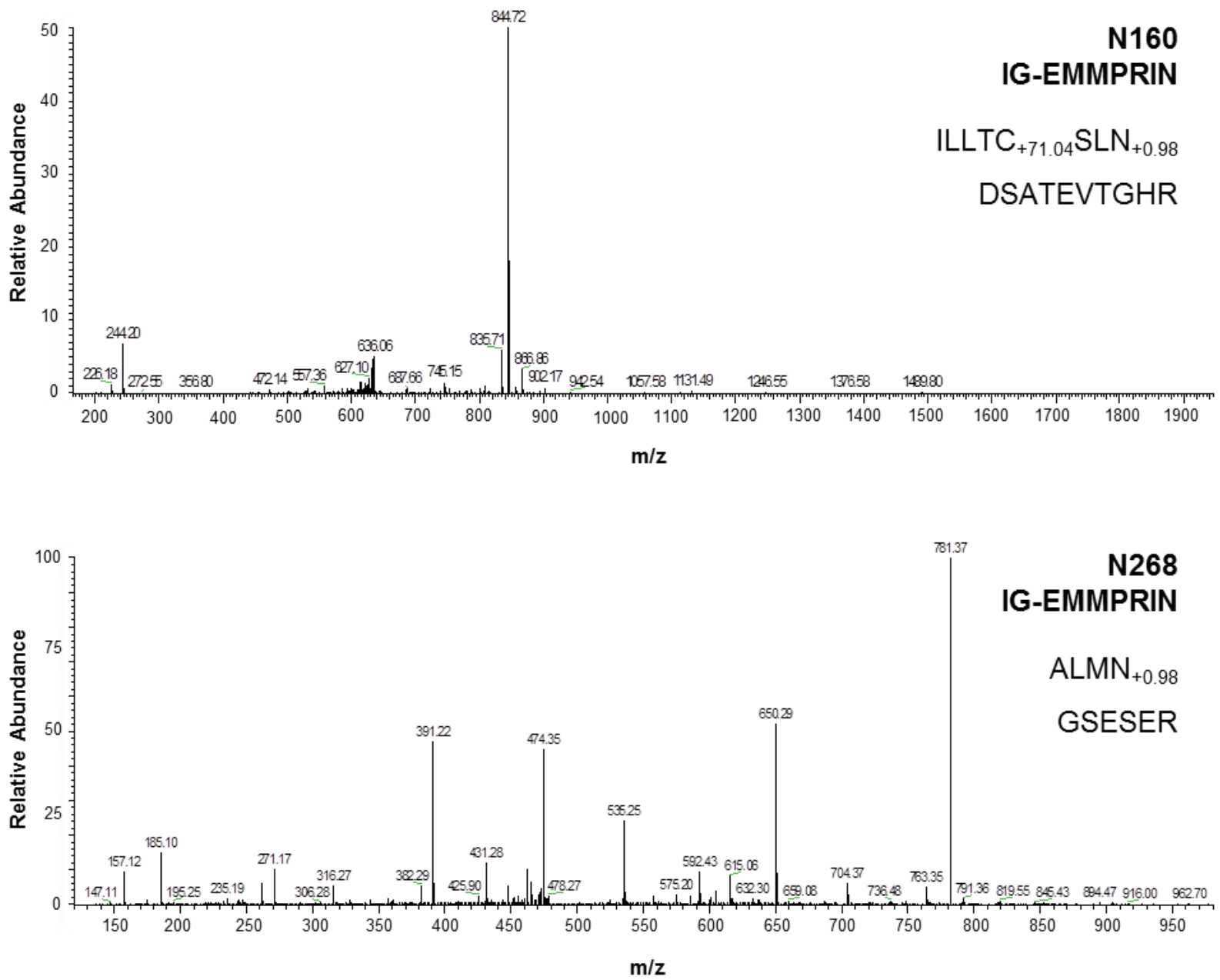

Fig. 47: MS/MS spectra

These images display the MS/MS spectra which were generated on the Orbitrap Velos mass spectrometer for the two identified peptides with deamidated N-residues which were detected in the IG-EMMPRIN spot. The

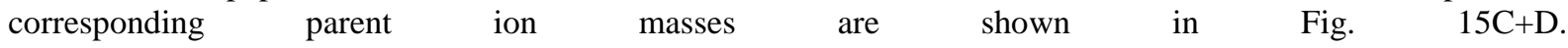




\section{Curriculum vitae}

Kerstin Menck

born on $11^{\text {th }}$ September 1986 in Hannover, Germany

Zimmermannstrasse 58, 37075 Göttingen, Germany

Phone: +49 (0) 551396197

E-mail: Kerstin.Menck@med.uni-goettingen.de

\section{EDUCATION AND RESEARCH EXPERIENCE}

10/2010 - present University Medical Center Göttingen, Dept. Hematology and Medical Oncology, Göttingen

Doctoral thesis: 'Extracellular vesicles as mediators of intercellular communication in human breast cancer progression'

10/2009 - 10/2010 Georg-August University Göttingen, Germany

M.Sc. Molecular Medicine

10/2006 - 09/2009 Georg-August University Göttingen, Germany

B.Sc. Molecular Medicine

08/2004 - 06/2006 Kurt-Schwitters-Gymnasium Misburg, Hannover, Germany

Abitur degree

08/1999-07/2004 Gymnasium Isernhagen, Isernhagen, Germany

High school

\section{AWARDS AND SCHOLARSHIPS}

2009

Scholarship for outstanding program achievements from the Ministry for Culture and Science of Lower Saxony

2006

Apollinaire award for outstanding achievements in French as a foreign language

\section{PUBLICATIONS}

Bleckmann, A., Leha, A., Artmann, S., Menck, K., Salinas-Riester, G., Binder, C., Pukrop, T., Beissbarth, T., Klemm, F. Integrated miRNA and mRNA profiling of breast cancer-educated macrophages identifies clinical subgroups, submitted.

Menck, K., Scharf, C., Bleckmann, A., Dyck, L., Pukrop, T., Wenzel, D., Binder, C., Klemm, F. Tumor-derived microvesicles mediate human breast cancer invasion through differentially glycosylated EMMPRIN, submitted.

Menck, K., Behme, D., Reiling, N., Binder, C., Pukrop, T., Klemm, F. Isolation of human monocytes by double gradient centrifugation and their differentiation to macrophages in Teflon-coated cell culture bags (2014), J Vis Exp, accepted. 
Menck, K., Klemm, F., Gross, J. C., Pukrop, T., Wenzel, D. Binder, C. Induction and transport of Wnt 5a during macrophage-induced malignant invasion is mediated by two types of extracellular vesicles (2013), OncoTarget 4 (11): 2057-66.

Rietkötter, E., Menck, K., Bleckmann, A., Farhat, K., Schaffrinski, M., Schulz, M., Hanisch, U.-K., Binder, C., Pukrop, T. Zoledronic acid inhibits macrophage/microglia-assisted breast cancer cell invasion (2013), OncoTarget 4 (9): 1449-1460.

Aung, T., Chapuy, B., Vogel, D., Wenzel, D., Oppermann, M., Lahmann, M., Weinhage, T., Menck, K., Hupfeld, T., Koch, R., Trümper, L., Wulf, G. G. Exosomal evasion of humoral immunotherapy in aggressive B-cell lymphoma modulated by ATP-binding cassette transporter A3 (2011), PNAS 108 (37): 15336-15341.

\section{PRESENTATIONS}

Menck, K., Klemm, F., Wenzel, D., Pukrop, T., Binder, C. Extracellular vesicles mediate Wnt 5a induction and transport during macrophage-induced malignant invasion (2013). Oral presentation at the Wnt symposium, German Cancer center, Heidelberg, Germany, July 14-16.

Menck, K., Pukrop, T., Dyck, L., Binder, C., Klemm, F. Tumor-derived microvesicles enhance EMMPRIN-dependent human breast cancer invasion (2013). Poster presentation at the Annual meeting of the International Society for Extracellular Vesicles, Boston, MA, April 17-20.

Menck, K., Klemm, F., Pukrop, T., Schulz, M., Binder, C. Plasma membrane-derived microvesicles mediate EMMPRIN-dependent human breast cancer invasion (2012). Oral presentation at the Annual Meeting of the German Society for Hematology\&Oncology, Stuttgart, Germany, October 19-23

Menck, K., Klemm, F., Pukrop, T., Schulz, M., Binder, C. Tumor cell-derived microvesicles enhance human breast cancer invasion through p38-MAPK (2012). Poster presentation at the Annual meeting of the International Society for Extracellular Vesicles, Gothenburg, Sweden, April 18-21.

Menck, K., Klemm, F., Schulz, M., Pukrop, T., Binder, C. Identification and further characterization of microparticle populations in microparticle-induced breast cancer invasion (2011). Poster presentation at the Annual Meeting of the German Society for Hematology\&Oncology, Basel, Switzerland, September 30 - October 04. 